\title{
A Comprehensive Investigation Toward the Indicative Proteins of Bladder Cancer in Urine: From Surveying Cellular Secretomes to Verifying Urine Proteins
}

Jiao Guo, ${ }^{\dagger, \downarrow}$ Yan Ren,${ }^{\S}$ Guixue Hou, ${ }^{\dagger, \downarrow}$ Bo Wen, ${ }^{\S}$ Feng Xian, ${ }^{\dagger,}$ Zhen Chen, ${ }^{\dagger}$ Ping Cui, ${ }^{\dagger}$

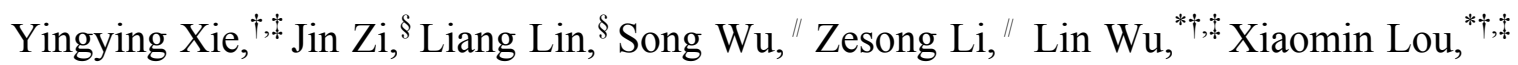
Siqi Liu, ${ }^{*+\ldots, \$}$

${ }^{\dagger}$ CAS Key Laboratory of Genome Sciences and Information, Beijing Institute of Genomics, Chinese Academy of Sciences, Beijing, 100101, China,

University of Chinese Academy of Sciences, Beijing, 100049, China,

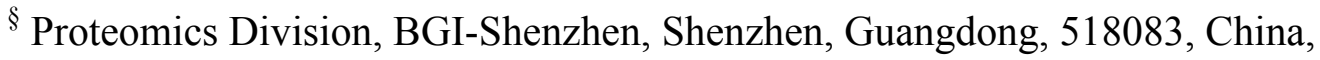
Shenzhen Second People's Hospital, Shenzhen, Guangdong, 518028, China.

Corresponding Authors:

Lin Wu, Tel and Fax: 86-10-84097465; E-mail: wul@big.ac.cn.

Xiaomin Lou, Tel and Fax: 86-10-84097465; E-mail: louxm@big.ac.cn.

Siqi Liu, Tel: 86-10-80485325; Fax: 86-10-80485324; E-mail: siqiliu@genomics.cn. 


\section{List of supplementary components}

The article contains Supplementary Figures: Fig. S1 to S3, and Supplementary Tables: Table S1 to S14.

Fig. S1. iTRAQ data quality control. (Page S5)

Fig. S2. MRM data quality control of synthesized peptides. (Page S6)

Fig. S3. Analysis of the unique peptide numbers of the target proteins. (Page S7)

Table S1. Clinical information of 47 urine samples (24 from normal controls and 23 from BCa patients). (Page S8- Page S9)

Table S2. Identification information of 108 proteins found by 2DE. (Page S10 - Page S16)

Table S3. Secretory characteristic analysis of the 108 proteins. (Page S17 - Page S19)

Table S4. 103 differential proteins from 5637 and T24 vs. SV-HUC-1 by 2DE. (Page S20 - Page S21)

Table S5. Information of all identified proteins through iTRAQ. (Page S22 - Page S52)

Table S6. 724 differential proteins quantified by 2DE and/or iTRAQ. (Page S53 - Page S66)

Table S7. Information of overlapped proteins identified by both 2DE and iTRAQ. (Page S67 - Page S68) 
Table S8. Identification information of 427 proteins detected in urine samples by SWATH. (Page S69 - Page S76)

Table S9. The list of urinary proteins screened by SWATH for MRM verification. (Page S77)

Table S10. Transitions list of the final 17 BCa related proteins for MRM. (Page S78 Page S81)

Table S11. Quantification results of the 17 BCa related proteins by MRM. (Page S82)

Table S12. ROC analysis results of the 10 potential BCa biomarkers. (Page S83)

Table S13. MRM quantification results of the 10 potential BCa biomarkers in peptide level. (Page S84)

Table S14. Disease-related information of the 10 potential BCa biomarkers summarized from references. (Page S85) 


\section{Supporting information of supplementary figures.}

Fig. S1. iTRAQ data quality control. (A) Overlap rates between the technical repeats for peptide and protein identification based on MS/MS. (B) Quantification correlation coefficiency for the identified proteins between two technical repeats based on tags. (C) Quantification correlation co-efficiency of 5637 vs. SV-HUC-1 and T24 vs. SV-HUC-1 for the identified proteins between the biological duplications.

Fig. S2. MRM data quality control of synthesized peptides. Eighteen peptides of the 7 proteins selected from the 10 potential BCa biomarkers were confirmed with synthesized peptides by MRM. Only 8 of them were illustrated here. Syn=the synthesized peptides spiked into urines, and urine $=$ the endogenous peptides in urine samples.

Fig. S3. Analysis of the unique peptide numbers of the target proteins. The median values of the unique peptides per protein of 5 groups were obtained from PeptideAtlas database. 1709 proteins: all the proteins identified by iTRAQ; 67 proteins: identified overlap proteins by both iTRAQ and 2DE; 41 proteins: only identified in 2DE and missed in iTRAQ; 25 proteins: retrieved proteins identified by label-free proteomics; 16 proteins: without any LC MS/MS signals in this study. 
Fig. $\mathbf{~} 1$

A.
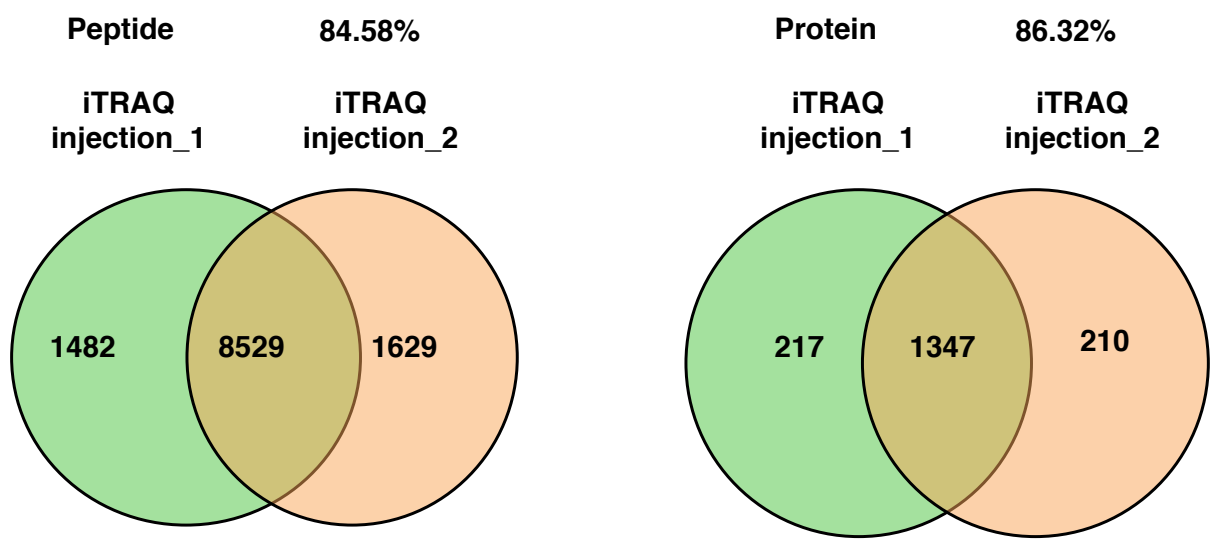

B. $115 / 117$

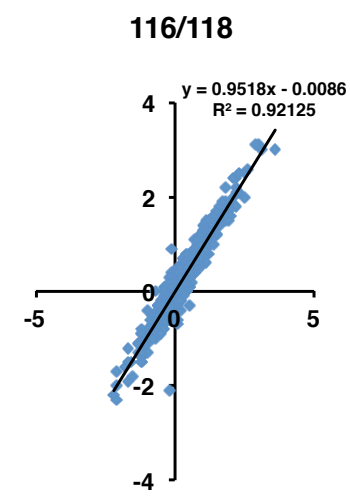

c.
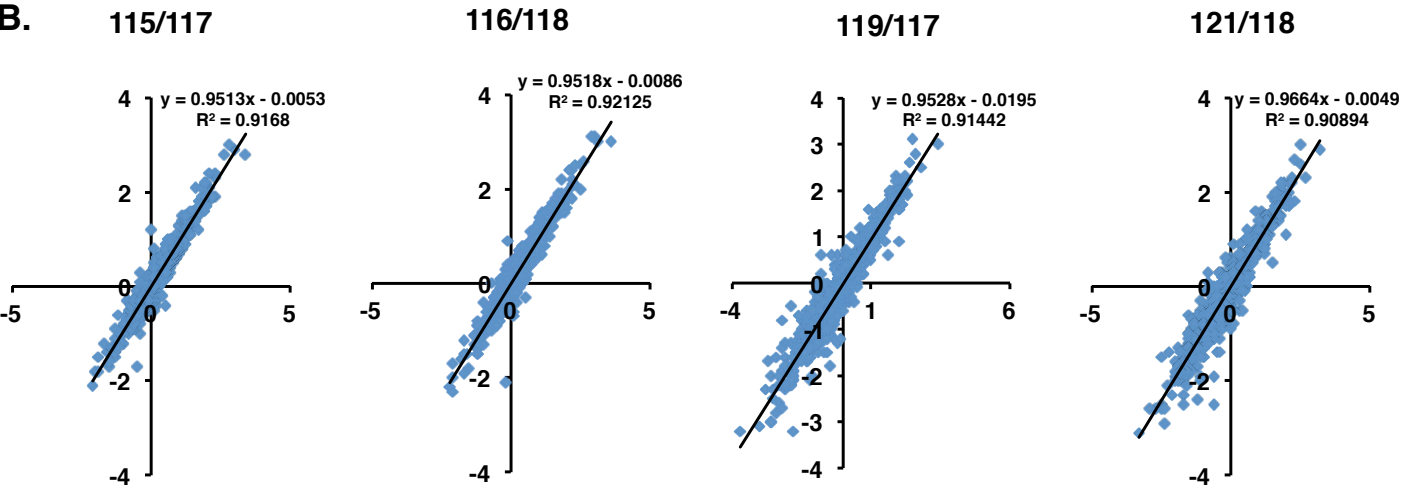

5637 vs. SV-HUC-1

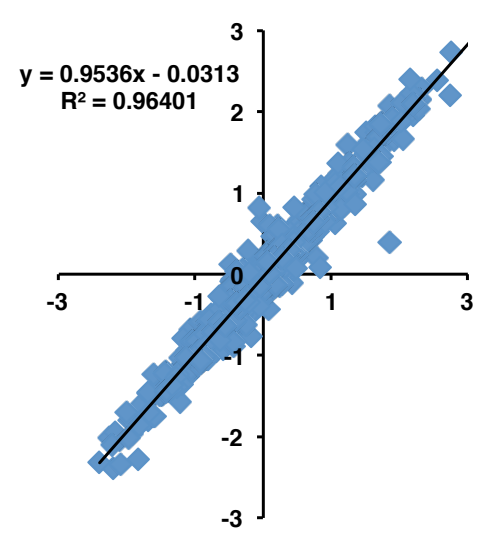

T24 vs. SV-HUC-1

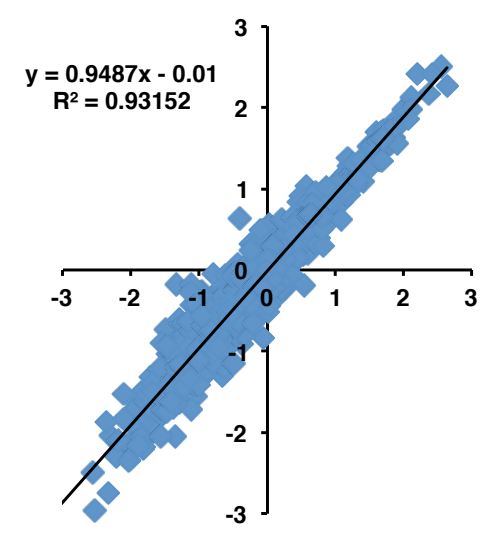


Fig. S2

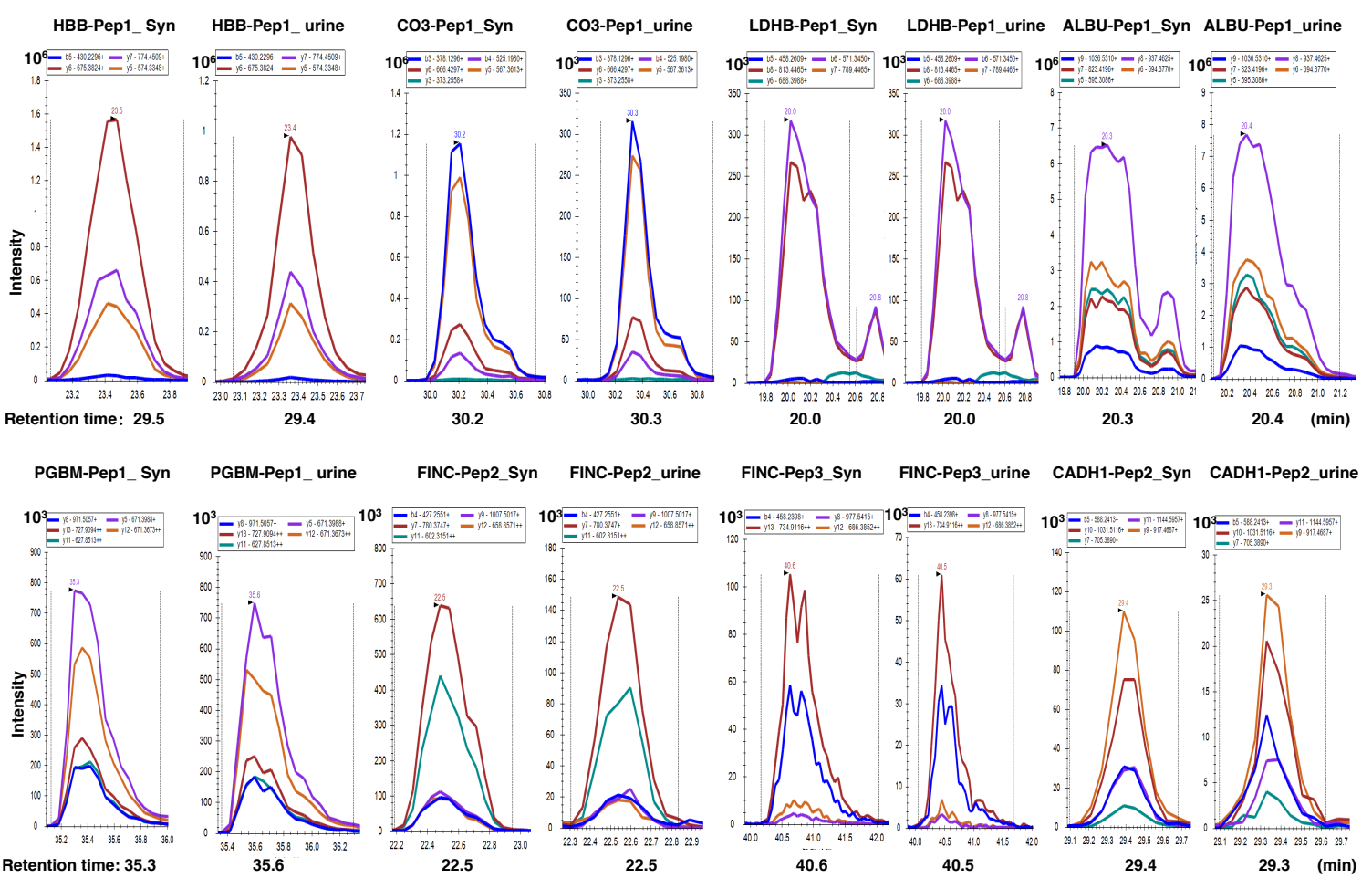


Fig. S3

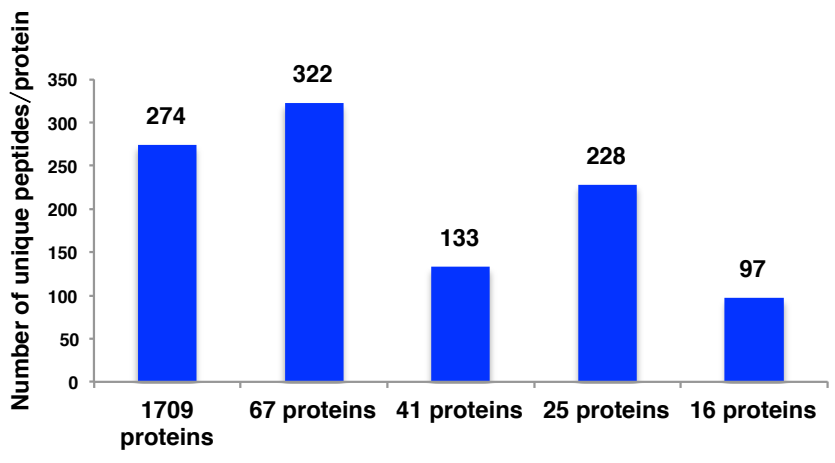




\section{Supporting information of supplementary tables.}

Table S1. Clinical information of 47 urine samples (24 from normal controls and 23 from BCa patiens).

\begin{tabular}{|c|c|c|c|c|c|c|}
\hline NO. & Gender & $\begin{array}{c}\text { Age } \\
\text { (years old) }\end{array}$ & $\begin{array}{c}\text { Sample } \\
\text { collection time }\end{array}$ & $\begin{array}{l}\text { Urine volume } \\
\qquad(\mathrm{mL})\end{array}$ & $\begin{array}{c}\text { Creatinine } \\
(\mu \mathrm{mol} / \mathrm{L}) \\
(\text { normal } \\
\text { range: } \\
53-110)\end{array}$ & $\begin{array}{c}\text { Urea nitrogen } \\
(\mathrm{mmol} / \mathrm{L}) \\
\text { (normal range: } \\
1.5-7.1)\end{array}$ \\
\hline B1 & Male & 78 & $09 / 06 / 2010$ & 100 & 136.6 & 7.41 \\
\hline B2 & Male & 57 & $09 / 08 / 2010$ & 100 & no test & no test \\
\hline B3 & Male & 53 & 09/09/2010 & 200 & 558.9 & 16.7 \\
\hline B4 & Male & 58 & $09 / 14 / 2010$ & 100 & 85.9 & 5.7 \\
\hline B5 & Male & 52 & $09 / 16 / 2010$ & 100 & 75 & 5.25 \\
\hline B6 & Male & 56 & $09 / 17 / 2010$ & 100 & 117 & 7.04 \\
\hline B7 & Male & 79 & $10 / 05 / 2010$ & 100 & 78.1 & 5.1 \\
\hline B8 & Female & 82 & $10 / 10 / 2010$ & 100 & 56 & 3.9 \\
\hline B9 & Male & 36 & $10 / 10 / 2010$ & 100 & 90.1 & 5.6 \\
\hline B10 & Female & 78 & $10 / 10 / 2010$ & 100 & 81.2 & 6.5 \\
\hline B11 & Male & 75 & $10 / 10 / 2010$ & 100 & 101 & 6.4 \\
\hline B12 & Male & 50 & $10 / 10 / 2010$ & 35 & 75.9 & 3.6 \\
\hline B13 & Male & 62 & $10 / 10 / 2010$ & 45 & 81.1 & 5.5 \\
\hline B14 & Male & 29 & $10 / 10 / 2010$ & 100 & 84 & 3.3 \\
\hline B15 & Male & 75 & $10 / 10 / 2010$ & 100 & 112.4 & 13.45 \\
\hline B16 & Female & 68 & $10 / 10 / 2010$ & 100 & 76 & 4.7 \\
\hline B17 & Male & 26 & $12 / 02 / 2010$ & 100 & no test & no test \\
\hline B18 & Male & 56 & $12 / 03 / 2011$ & 100 & 104.5 & 5.82 \\
\hline B19 & Male & 74 & $09 / 06 / 2010$ & 100 & 127.3 & 5.26 \\
\hline $\mathrm{B} 20$ & Male & 36 & $09 / 11 / 2010$ & 200 & 308.9 & 14.24 \\
\hline $\mathrm{B} 21$ & Male & 36 & $10 / 20 / 2010$ & 100 & 88.6 & 4.95 \\
\hline B22 & Male & 67 & $09 / 21 / 2010$ & 100 & 103 & 5 \\
\hline B23 & Female & 40 & $09 / 28 / 2010$ & 100 & 98 & 3.63 \\
\hline N1 & Male & 61 & $09 / 16 / 2010$ & 100 & 97.9 & 4.48 \\
\hline N2 & Male & 34 & $09 / 28 / 2010$ & 100 & 121.5 & 5.46 \\
\hline N3 & Male & 34 & $09 / 28 / 2010$ & 100 & 92.1 & 4.82 \\
\hline N4 & Male & 67 & $09 / 28 / 2010$ & 100 & 68.9 & 5.8 \\
\hline N5 & Male & 71 & $10 / 11 / 2010$ & 100 & no test & no test \\
\hline N6 & Male & 71 & $10 / 11 / 2010$ & 100 & no test & no test \\
\hline N7 & Male & 82 & $10 / 11 / 2010$ & 100 & no test & no test \\
\hline N8 & Female & 55 & $10 / 14 / 2010$ & 100 & 76.2 & 5.72 \\
\hline N9 & Female & 47 & $10 / 14 / 2010$ & 100 & 60.2 & 3.89 \\
\hline N10 & Male & 55 & $10 / 14 / 2010$ & 75 & 84.8 & 5.02 \\
\hline N11 & Male & 43 & $10 / 15 / 2010$ & 100 & no test & no test \\
\hline N12 & Male & 29 & $10 / 19 / 2010$ & 100 & no test & no test \\
\hline N13 & Male & 69 & $10 / 20 / 2010$ & 100 & 94.8 & 8.42 \\
\hline N14 & Male & 81 & $10 / 20 / 2010$ & 100 & 104.1 & 5.73 \\
\hline N15 & Male & 30 & $10 / 21 / 2010$ & 100 & no test & no test \\
\hline N16 & Male & 42 & $10 / 21 / 2010$ & 100 & no test & no test \\
\hline N17 & Male & 64 & $10 / 26 / 2010$ & 90 & 98.3 & 5.66 \\
\hline N18 & Male & 73 & $10 / 26 / 2010$ & 90 & 74.3 & 4.64 \\
\hline N19 & Male & 53 & $10 / 26 / 2010$ & 100 & 92.1 & 3 \\
\hline
\end{tabular}




\begin{tabular}{lcccccc} 
N20 & Female & 33 & $12 / 30 / 2014$ & 80 & 84.5 & no test \\
N21 & Female & 62 & $12 / 30 / 2014$ & 90 & 81.7 & no test \\
N22 & Male & 41 & $12 / 30 / 2014$ & 100 & 67 & no test \\
N23 & Male & 26 & $12 / 30 / 2014$ & 90 & 48 & no test \\
N24 & Male & 42 & $12 / 30 / 2014$ & 100 & 72 & no test \\
\hline
\end{tabular}


Table S2. Identification information of 108 proteins found by 2DE

\begin{tabular}{|c|c|c|c|c|c|c|c|c|}
\hline $\begin{array}{l}\text { Spot } \\
\text { no. }\end{array}$ & $\begin{array}{c}\text { Protein } \\
\text { no. }\end{array}$ & $\begin{array}{l}\text { Swiss-prot } \\
\text { Number }\end{array}$ & $\begin{array}{l}\text { Protein } \\
\text { name }\end{array}$ & $\begin{array}{c}\text { MW } \\
\text { (The. /Exp.) }\end{array}$ & $\begin{array}{c}\text { PI } \\
\text { (The. /Exp.) }\end{array}$ & $\begin{array}{c}\text { Sequence } \\
\text { coverage } \\
(\%)\end{array}$ & Score & Sample \\
\hline S1 & $\overline{P 1}$ & P31947 & $1433 \mathrm{~S}$ & $27.87 / 31$ & $4.68 / 4.71$ & $13 \%$ & 213 & SV \\
\hline S2 & P2 & O95336 & 6PGL & $27.82 / 29$ & $5.71 / 5.92$ & $16 \%$ & 222 & SV \\
\hline S3 & P3 & P05067 & A4 & $87.9 / 27$ & $4.73 / 4.57$ & $11 \%$ & 62 & T24 \\
\hline S4 & P4 & P62736 & ACTA & $42.05 / 46$ & $5.23 / 5.44$ & $13 \%$ & 56 & T24 \\
\hline S5 & P4 & P62736 & ACTA & $42.05 / 46$ & $5.23 / 5.41$ & $5 \%$ & 59 & $\mathrm{~T} 24$ \\
\hline S6 & P4 & P62736 & ACTA & $42.05 / 16$ & $5.23 / 5.86$ & $14 \%$ & 103 & T24 \\
\hline S7 & P4 & P62736 & ACTA & $42.05 / 28$ & $5.23 / 5.64$ & $19 \%$ & 64 & 5637 \\
\hline S8 & P4 & P62736 & ACTA & $42.05 / 32$ & $5.23 / 5.17$ & $15 \%$ & 68 & 5637 \\
\hline S9 & P4 & P62736 & ACTA & $42.05 / 47$ & $5.23 / 5.32$ & $14 \%$ & 67 & 5637 \\
\hline $\mathrm{S} 10$ & P4 & P62736 & ACTA & $42.38 / 43$ & $5.23 / 5.63$ & $17 \%$ & 114 & SV \\
\hline S11 & P5 & P60709 & АCTB & $42.05 / 47$ & $5.29 / 5.49$ & $9 \%$ & 114 & T24 \\
\hline S12 & P5 & P60709 & АCTB & $42.05 / 39$ & $5.29 / 5.61$ & $12 \%$ & 122 & T24 \\
\hline $\mathrm{S} 13$ & P5 & P60709 & АCTB & $42 / 16$ & $5.29 / 7.16$ & $7 \%$ & 154 & T24 \\
\hline $\mathrm{S} 14$ & P5 & P60709 & АCTB & $42.05 / 46$ & $5.29 / 5.10$ & $15 \%$ & 259 & 5637 \\
\hline S15 & P5 & P60709 & АCTB & $42.05 / 46$ & $5.29 / 5.19$ & $18 \%$ & 291 & 5637 \\
\hline S16 & P5 & P60709 & АCTB & $42.05 / 44$ & $5.29 / 5.64$ & $7 \%$ & 61 & 5637 \\
\hline S17 & P5 & P60709 & АCTB & $42.05 / 32$ & $5.29 / 5.36$ & $10 \%$ & 127 & 5637 \\
\hline $\mathrm{S} 18$ & P5 & P60709 & АCTB & $42.05 / 33$ & $5.29 / 4.32$ & $15 \%$ & 192 & SV \\
\hline S19 & P5 & P60709 & АCTB & $42.05 / 45$ & $5.29 / 5.52$ & $10 \%$ & 199 & SV \\
\hline S20 & P5 & P60709 & АCTB & $42.05 / 43$ & $5.29 / 5.38$ & $13 \%$ & 250 & SV \\
\hline $\mathrm{S} 21$ & P5 & P60709 & АCTB & $42.05 / 43$ & $5.29 / 5.44$ & $10 \%$ & 261 & SV \\
\hline S22 & P5 & P60709 & АCTB & $42.05 / 43$ & $5.29 / 5.51$ & $18 \%$ & 420 & SV \\
\hline S23 & P6 & O00468 & AGRIN & $22.86 / 26$ & $4.97 / 5.51$ & $15 \%$ & 56 & T24 \\
\hline S24 & P7 & P02768 & ALBU & $71.32 / 28$ & $5.67 / 8.60$ & $16 \%$ & 146 & 5637 \\
\hline $\mathrm{S} 25$ & P7 & P02768 & ALBU & $71.32 / 25$ & $5.67 / 10$ & $14 \%$ & 66 & 5637 \\
\hline S26 & P7 & P02768 & ALBU & $71.32 / 39$ & $5.67 / 4.71$ & $17 \%$ & 201 & 5637 \\
\hline S27 & P7 & P02768 & ALBU & $71.32 / 39$ & $5.67 / 5.41$ & $9 \%$ & 260 & 5637 \\
\hline S28 & P7 & P02768 & ALBU & $71.32 / 27$ & $5.67 / 6.03$ & $12 \%$ & 344 & 5637 \\
\hline S29 & P7 & P02768 & ALBU & $71.32 / 27$ & $5.10 / 6.03$ & $11 \%$ & 252 & 5637 \\
\hline $\mathrm{S} 30$ & P7 & P02768 & ALBU & $71.32 / 34$ & $5.67 / 5.96$ & $12 \%$ & 94 & SV \\
\hline $\mathrm{S} 31$ & P8 & P05091 & ALDH2 & $56.86 / 51$ & $5.69 / 6.03$ & $14 \%$ & 87 & SV \\
\hline $\mathrm{S} 32$ & P9 & P04075 & ALDOA & $39.85 / 42$ & $8.3 / 8.76$ & $15 \%$ & 39 & $\mathrm{~T} 24$ \\
\hline S33 & P9 & P04075 & ALDOA & $39.85 / 39$ & $8.3 / 9.10$ & $12 \%$ & 154 & T24 \\
\hline S34 & P9 & P04075 & ALDOA & $39.85 / 39$ & $8.3 / 9.33$ & $13 \%$ & 122 & T24 \\
\hline S35 & P9 & P04075 & ALDOA & $39.85 / 39$ & $8.3 / 9.67$ & $13 \%$ & 64 & T24 \\
\hline S36 & P9 & P04075 & ALDOA & $39.85 / 42$ & $8.3 / 10$ & $13 \%$ & 66 & SV \\
\hline S37 & P9 & P04075 & ALDOA & $39.85 / 40$ & $8.39 / 7.42$ & $9 \%$ & 119 & SV \\
\hline S38 & P10 & P15121 & ALDR & $36.23 / 36$ & $6.55 / 6.94$ & $7 \%$ & 104 & SV \\
\hline S39 & P10 & P15121 & ALDR & $36.2 / 37$ & $6.55 / 7.78$ & $17 \%$ & 150 & SV \\
\hline S40 & P11 & P39687 & AN32A & $28.68 / 31$ & $3.98 / 3.78$ & $14 \%$ & 181 & SV \\
\hline S41 & P12 & P01008 & ANT3 & $53.05 / 57$ & $6.32 / 4.75$ & $12 \%$ & 60 & $\mathrm{~T} 24$ \\
\hline S42 & P13 & P02649 & APOE & $36.25 / 30$ & $6.02 / 7.02$ & $14 \%$ & 67 & T24 \\
\hline S43 & P14 & P05089 & ARGI1 & $34.88 / 38$ & $6.72 / 8.56$ & $15 \%$ & 66 & SV \\
\hline S44 & P14 & P05089 & ARGI1 & $34.88 / 39$ & $6.72 / 7.03$ & $12 \%$ & 88 & SV \\
\hline S45 & P15 & P61769 & B2MG & $13.8 / 13$ & $6.06 / 6.06$ & $8 \%$ & 61 & T24 \\
\hline S46 & P15 & P61769 & B2MG & $13.8 / 13.8$ & $6.06 / 7.15$ & $36 \%$ & 86 & T24 \\
\hline S47 & P15 & P61769 & B2MG & $13.82 / 12$ & $6.06 / 6.06$ & $16 \%$ & 105 & 5637 \\
\hline $\mathrm{S} 48$ & P15 & P61769 & B2MG & $13.82 / 12$ & $6.06 / 6.06$ & $16 \%$ & 61 & 5637 \\
\hline S49 & P15 & P61769 & B2MG & $13.82 / 12$ & $6.06 / 8.30$ & $8 \%$ & 66 & 5637 \\
\hline S50 & P15 & P61769 & B2MG & $13.82 / 12$ & $6.06 / 8.31$ & $13 \%$ & 60 & 5637 \\
\hline
\end{tabular}




\begin{tabular}{|c|c|c|c|c|c|c|c|c|}
\hline S51 & $\mathrm{P} 15$ & P61769 & $\mathrm{B} 2 \mathrm{MG}$ & $13.82 / 12$ & $6.06 / 9.13$ & $7 \%$ & 70 & 5637 \\
\hline S52 & P15 & P61769 & B2MG & $13.82 / 12$ & $6.06 / 6.22$ & $8 \%$ & 67 & SV \\
\hline S53 & P15 & P61769 & B2MG & $13.82 / 12$ & $6.06 / 6.06$ & $8 \%$ & 72 & SV \\
\hline S54 & P16 & P27797 & CALR & $48.28 / 63$ & $4.29 / 4.29$ & $12 \%$ & 79 & T24 \\
\hline S55 & P16 & P27797 & CALR & $48.28 / 60$ & $4.29 / 4.29$ & $6 \%$ & 138 & 5637 \\
\hline S56 & P16 & P27797 & CALR & $48.28 / 34$ & $4.29 / 4.96$ & $12 \%$ & 73 & 5637 \\
\hline S57 & P16 & P27797 & CALR & $48.28 / 54$ & $4.29 / 4.29$ & $21 \%$ & 120 & SV \\
\hline S58 & P17 & P04040 & CATA & $59.95 / 60$ & $6.95 / 8.48$ & $14 \%$ & 79 & SV \\
\hline S59 & P18 & P07339 & CATD & $45.04 / 54$ & $6.1 / 4.62$ & $13 \%$ & 78 & 5637 \\
\hline S60 & P18 & P07339 & CATD & $45.04 / 53$ & $6.1 / 4.73$ & $15 \%$ & 93 & 5637 \\
\hline S61 & P18 & P07339 & CATD & $45.04 / 48$ & $6.1 / 6.08$ & $12 \%$ & 71 & SV \\
\hline S62 & P18 & P07339 & CATD & $45.04 / 45$ & $6.1 / 5.95$ & $12 \%$ & 71 & SV \\
\hline S63 & P18 & P07339 & CATD & $45.04 / 45$ & $6.1 / 5.78$ & $18 \%$ & 155 & SV \\
\hline S64 & P18 & P07339 & CATD & $45 / 45$ & $6.1 / 5.63$ & $9 \%$ & 140 & SV \\
\hline S65 & P19 & P10809 & CH60 & $61.92 / 61$ & $5.24 / 5.70$ & $15 \%$ & 136 & SV \\
\hline S66 & $\mathrm{P} 20$ & P23528 & COF1 & $18.7 / 19$ & $8.26 / 8.40$ & $11 \%$ & 106 & 5637 \\
\hline S67 & $\mathrm{P} 20$ & P23528 & COF1 & $18.72 / 19$ & $8.26 / 10$ & $18 \%$ & 213 & SV \\
\hline S68 & P21 & P00167 & CYB5 & $15.32 / 18$ & $4.86 / 4.87$ & $9 \%$ & 78 & SV \\
\hline S69 & $\mathrm{P} 22$ & P01034 & CYTC & $16 / 15$ & $9 / 10.00$ & $36 \%$ & 302 & $\mathrm{~T} 24$ \\
\hline S70 & $\mathrm{P} 23$ & Q30KQ2 & DB130 & $6.30 / 19$ & $9.06 / 5.46$ & $13 \%$ & 72 & 5637 \\
\hline S71 & $\mathrm{P} 24$ & Q15392 & DHC24 & $61.8 / 22$ & $8.16 / 6.13$ & $12 \%$ & 73 & $\mathrm{~T} 24$ \\
\hline S72 & P25 & Q18PE1 & DOK7 & $53.81 / 48$ & $5.99 / 7.64$ & $9 \%$ & 65 & T24 \\
\hline S73 & P25 & Q18PE1 & DOK7 & $53.81 / 45$ & $5.99 / 7.64$ & $10 \%$ & 75 & T24 \\
\hline S74 & $\mathrm{P} 25$ & Q18PE1 & DOK7 & $53.81 / 44$ & $5.99 / 7.66$ & $12 \%$ & 73 & T24 \\
\hline S75 & $\mathrm{P} 25$ & Q18PE1 & DOK7 & $53.81 / 27$ & $5.99 / 8.15$ & $19 \%$ & 64 & T24 \\
\hline S76 & $\mathrm{P} 25$ & Q18PE1 & DOK7 & $53.81 / 27$ & $5.99 / 6.58$ & $11 \%$ & 84 & $\mathrm{~T} 24$ \\
\hline S77 & P25 & Q18PE1 & DOK7 & $53.81 / 26$ & $5.99 / 6.52$ & $10 \%$ & 89 & $\mathrm{~T} 24$ \\
\hline S78 & $\mathrm{P} 25$ & Q18PE1 & DOK7 & $53.81 / 33$ & $5.99 / 7.30$ & $9 \%$ & 83 & T24 \\
\hline S79 & $\mathrm{P} 25$ & Q18PE1 & DOK7 & $53.81 / 26$ & $5.99 / 5.80$ & $10 \%$ & 81 & T24 \\
\hline $\mathrm{S} 80$ & P25 & Q18PE1 & DOK7 & $53.81 / 23$ & $5.99 / 6.10$ & $13 \%$ & 62 & T24 \\
\hline S81 & P25 & Q18PE1 & DOK7 & $53.81 / 29$ & $5.99 / 4.46$ & $12 \%$ & 75 & T24 \\
\hline S82 & P25 & Q18PE1 & DOK7 & $53.81 / 30$ & $5.99 / 4.75$ & $11 \%$ & 73 & $\mathrm{~T} 24$ \\
\hline S83 & P25 & Q18PE1 & DOK7 & $53.81 / 64$ & $5.99 / 5.88$ & $12 \%$ & 61 & $\mathrm{~T} 24$ \\
\hline S84 & $\mathrm{P} 25$ & Q18PE1 & DOK7 & $53.81 / 31$ & $5.99 / 7.47$ & $13 \%$ & 82 & $\mathrm{~T} 24$ \\
\hline S85 & P25 & Q18PE1 & DOK7 & $53.81 / 15$ & $5.99 / 5.38$ & $9 \%$ & 88 & 5637 \\
\hline S86 & P25 & Q18PE1 & DOK7 & $53.81 / 12$ & $5.99 / 5.99$ & $10 \%$ & 72 & 5637 \\
\hline S87 & P25 & Q18PE1 & DOK7 & $53.81 / 14$ & $5.99 / 5.96$ & $13 \%$ & 76 & 5637 \\
\hline S88 & P25 & Q18PE1 & DOK7 & $53.81 / 13$ & $5.99 / 5.90$ & $15 \%$ & 84 & 5637 \\
\hline S89 & P25 & Q18PE1 & DOK7 & $53.81 / 15$ & $5.99 / 6.73$ & $13 \%$ & 61 & 5637 \\
\hline S90 & P25 & Q18PE1 & DOK7 & $53.81 / 14$ & $5.99 / 6.06$ & $13 \%$ & 65 & 5637 \\
\hline S91 & P25 & Q18PE1 & DOK7 & $53.81 / 13$ & $5.99 / 6.04$ & $14 \%$ & 78 & 5637 \\
\hline S92 & P25 & Q18PE1 & DOK7 & $53.81 / 15$ & $5.99 / 9.48$ & $15 \%$ & 82 & 5637 \\
\hline S93 & $\mathrm{P} 25$ & Q18PE1 & DOK7 & $53.81 / 27$ & $5.99 / 8.34$ & $13 \%$ & 73 & 5637 \\
\hline S94 & $\mathrm{P} 25$ & Q18PE1 & DOK7 & $53.81 / 23$ & $5.99 / 10$ & $15 \%$ & 92 & 5637 \\
\hline S95 & P25 & Q18PE1 & DOK7 & $53.81 / 39$ & $5.99 / 5.28$ & $13 \%$ & 61 & 5637 \\
\hline S96 & P25 & Q18PE1 & DOK7 & $53.81 / 39$ & $5.99 / 8.47$ & $18 \%$ & 68 & 5637 \\
\hline S97 & $\mathrm{P} 26$ & P06733 & ENOA & $47.48 / 55$ & $7.01 / 7.01$ & $14 \%$ & 179 & $\mathrm{~T} 24$ \\
\hline S98 & $\mathrm{P} 26$ & P06733 & ENOA & $47.48 / 56$ & $7.01 / 7.19$ & $18 \%$ & 268 & $\mathrm{~T} 24$ \\
\hline S99 & P26 & P06733 & ENOA & $47.48 / 55$ & $7.01 / 7.35$ & $17 \%$ & 328 & $\mathrm{~T} 24$ \\
\hline S100 & P26 & P06733 & ENOA & $47.48 / 54$ & $7.01 / 7.53$ & $14 \%$ & 298 & $\mathrm{~T} 24$ \\
\hline S101 & $\mathrm{P} 26$ & P06733 & ENOA & $47.48 / 53$ & $7.01 / 6.03$ & $14 \%$ & 101 & 5637 \\
\hline S102 & $\mathrm{P} 26$ & P06733 & ENOA & $47.48 / 53$ & $7.01 / 6.27$ & $13 \%$ & 122 & 5637 \\
\hline S103 & P26 & P06733 & ENOA & $47.48 / 53$ & $7.01 / 7.49$ & $17 \%$ & 122 & 5637 \\
\hline S104 & $\mathrm{P} 26$ & P06733 & ENOA & $47.48 / 53$ & $7.01 / 8.20$ & $8 \%$ & 67 & 5637 \\
\hline S105 & $\mathrm{P} 26$ & P06733 & ENOA & $47.48 / 43$ & $7.01 / 6.88$ & $7 \%$ & 69 & 5637 \\
\hline S106 & $\mathrm{P} 26$ & P06733 & ENOA & $47.48 / 44$ & $7.01 / 7.56$ & $17 \%$ & 171 & SV \\
\hline
\end{tabular}




\begin{tabular}{|c|c|c|c|c|c|c|c|c|}
\hline S107 & P27 & P16930 & FAAA & $46.74 / 42$ & $6.45 / 6.07$ & $12 \%$ & 79 & SV \\
\hline S108 & P28 & P02751 & FINC & $26.60 / 94$ & $5.39 / 4.31$ & $11 \%$ & 117 & 5637 \\
\hline S109 & P28 & P02751 & FINC & $26.60 / 77$ & $5.39 / 4.72$ & $10 \%$ & 83 & 5637 \\
\hline S110 & P28 & P02751 & FINC & $26.60 / 60$ & $5.39 / 4.63$ & $15 \%$ & 61 & 5637 \\
\hline S111 & P28 & P02751 & FINC & $26.60 / 57$ & $5.39 / 4.55$ & $13 \%$ & 65 & 5637 \\
\hline S112 & P28 & P02751 & FINC & $26.6 / 53$ & $5.39 / 4.89$ & $11 \%$ & 79 & SV \\
\hline S113 & P29 & Q13283 & G3BP1 & $52.19 / 25$ & $5.36 / 5.65$ & $11 \%$ & 63 & SV \\
\hline S114 & P30 & P04406 & G3P & $36.20 / 39$ & $8.57 / 7.95$ & $12 \%$ & 64 & $\mathrm{~T} 24$ \\
\hline S115 & P30 & P04406 & G3P & $36.20 / 39$ & $8.57 / 8.17$ & $8 \%$ & 104 & $\mathrm{~T} 24$ \\
\hline S116 & $\mathrm{P} 30$ & P04406 & G3P & $36.20 / 36$ & $8.57 / 8.60$ & $12 \%$ & 177 & $\mathrm{~T} 24$ \\
\hline S117 & P30 & P04406 & G3P & $36.20 / 39$ & $8.57 / 9.26$ & $8 \%$ & 165 & $\mathrm{~T} 24$ \\
\hline S118 & P30 & P04406 & G3P & $36.20 / 39$ & $8.57 / 9.66$ & $12 \%$ & 182 & $\mathrm{~T} 24$ \\
\hline S119 & P30 & P04406 & G3P & $36.20 / 39$ & $8.57 / 10.00$ & $8 \%$ & 65 & $\mathrm{~T} 24$ \\
\hline S120 & P30 & P04406 & G3P & $36.20 / 39$ & $8.57 / 9.45$ & $8 \%$ & 61 & $\mathrm{~T} 24$ \\
\hline S121 & P30 & P04406 & G3P & $36.20 / 39$ & $8.57 / 7.75$ & $8 \%$ & 62 & $\mathrm{~T} 24$ \\
\hline S122 & P30 & P04406 & G3P & $36.20 / 30$ & $8.57 / 7.10$ & $8 \%$ & 82 & T24 \\
\hline $\mathrm{S} 123$ & P31 & P78417 & GSTO1 & $27.83 / 32$ & $6.23 / 5.81$ & $9 \%$ & 60 & $\mathrm{~T} 24$ \\
\hline S124 & P32 & P09211 & GSTP1 & $23.57 / 28$ & $5.43 / 5.64$ & $15 \%$ & 65 & $\mathrm{~T} 24$ \\
\hline S125 & P32 & P09211 & GSTP1 & $23.57 / 25$ & $5.43 / 5.65$ & $7 \%$ & 103 & $\mathrm{~T} 24$ \\
\hline S126 & P32 & P09211 & GSTP1 & $23.57 / 19$ & $5.43 / 4.73$ & $12 \%$ & 77 & 5637 \\
\hline S127 & P32 & P09211 & GSTP1 & $23.57 / 26$ & $5.43 / 5.42$ & $12 \%$ & 87 & 5637 \\
\hline $\mathrm{S} 128$ & P32 & P09211 & GSTP1 & $23.57 / 26$ & $5.43 / 5.14$ & $12 \%$ & 69 & 5637 \\
\hline S129 & P32 & P09211 & GSTP1 & $23.57 / 26$ & $5.43 / 5.71$ & $12 \%$ & 204 & SV \\
\hline S130 & P32 & P09211 & GSTP1 & $23.57 / 25$ & $5.43 / 5.71$ & $22 \%$ & 258 & SV \\
\hline S131 & P32 & P09211 & GSTP1 & $23.57 / 28$ & $5.43 / 5.62$ & $15 \%$ & 92 & SV \\
\hline S132 & P32 & P09211 & GSTP1 & $23.57 / 26$ & $5.43 / 5.61$ & $9 \%$ & 94 & SV \\
\hline S133 & P32 & P09211 & GSTP1 & $23.57 / 27$ & $5.43 / 5.48$ & $11 \%$ & 93 & SV \\
\hline S134 & P32 & P09211 & GSTP1 & $23.57 / 27$ & $5.43 / 5.34$ & $12 \%$ & 203 & SV \\
\hline S135 & P32 & P09211 & GSTP1 & $23.57 / 26$ & $5.43 / 5.51$ & $22 \%$ & 258 & SV \\
\hline S136 & P32 & P09211 & GSTP1 & $23.57 / 30$ & $5.43 / 5.45$ & $12 \%$ & 92 & SV \\
\hline S137 & P33 & Q96QV6 & $\mathrm{H} 2 \mathrm{~A} 1 \mathrm{~A}$ & $14.23 / 15$ & $10.86 / 4.85$ & $16 \%$ & 95 & SV \\
\hline S138 & P34 & Q96A08 & H2B1A & $14.16 / 17$ & $10.32 / 5.52$ & $12 \%$ & 63 & SV \\
\hline S139 & P35 & P33778 & H2B1B & $13.94 / 17$ & $10.32 / 4.33$ & $19 \%$ & 72 & 5637 \\
\hline S140 & P36 & P62805 & H4 & $11.36 / 14$ & $11.36 / 4.29$ & $17 \%$ & 72 & SV \\
\hline S141 & P37 & P68871 & HBB & $16.1 / 13$ & $6.75 / 7.65$ & $21 \%$ & 69 & $\mathrm{~T} 24$ \\
\hline S142 & P38 & P04792 & HSPB1 & $22.83 / 28$ & $5.98 / 5.85$ & $17 \%$ & 194 & SV \\
\hline S143 & P38 & P04792 & HSPB1 & $22.83 / 28$ & $5.98 / 6.07$ & $13 \%$ & 237 & SV \\
\hline S144 & P38 & P04792 & HSPB1 & $22.83 / 29$ & $5.98 / 6.02$ & $13 \%$ & 157 & SV \\
\hline S145 & P38 & P04792 & HSPB1 & $22.83 / 29$ & $5.98 / 5.95$ & $8 \%$ & 67 & SV \\
\hline S146 & P38 & P04792 & HSPB1 & $22.83 / 27$ & $5.98 / 5.94$ & $7 \%$ & 64 & SV \\
\hline S147 & P38 & P04792 & HSPB1 & $22.83 / 28$ & $5.98 / 6.00$ & $14 \%$ & 67 & SV \\
\hline S148 & P39 & P12277 & KCRB & $42.9 / 46$ & $5.35 / 7.10$ & $13 \%$ & 62 & $\mathrm{~T} 24$ \\
\hline S149 & P39 & P12277 & KCRB & $42.9 / 45$ & $5.35 / 7.20$ & $7 \%$ & 66 & $\mathrm{~T} 24$ \\
\hline S150 & P40 & P06732 & KCRM & $43.3 / 45$ & $6.77 / 7.25$ & $11 \%$ & 75 & $\mathrm{~T} 24$ \\
\hline S151 & P41 & Q92876 & KLK6 & $27.52 / 34$ & $6.91 / 6.26$ & $17 \%$ & 100 & SV \\
\hline S152 & P41 & Q92876 & KLK6 & $27.52 / 34$ & $6.91 / 6.96$ & $25 \%$ & 165 & SV \\
\hline S153 & P41 & Q92876 & KLK6 & $27.52 / 33$ & $6.91 / 6.24$ & $14 \%$ & 68 & SV \\
\hline S154 & P41 & Q92876 & KLK6 & $27.52 / 33$ & $6.91 / 7.06$ & $13 \%$ & 210 & SV \\
\hline S155 & P41 & Q92876 & KLK6 & $27.52 / 32$ & $6.91 / 7.80$ & $13 \%$ & 172 & SV \\
\hline S156 & P41 & Q92876 & KLK6 & $27.52 / 33$ & $6.91 / 6.08$ & $10 \%$ & 81 & SV \\
\hline S157 & P42 & P14618 & KPYM & $58.47 / 26$ & 7.95/7.74 & $12 \%$ & 79 & SV \\
\hline S158 & P43 & P11047 & LAMC1 & $18.32 / 46$ & $4.94 / 5.93$ & $11 \%$ & 75 & 5637 \\
\hline S159 & P44 & P09382 & LEG1 & $15 / 15$ & $5.34 / 5.34$ & $26 \%$ & 228 & $\mathrm{~T} 24$ \\
\hline S160 & P44 & P09382 & LEG1 & $15 / 18$ & $5.34 / 7.39$ & $16 \%$ & 74 & $\mathrm{~T} 24$ \\
\hline S161 & $\mathrm{P} 44$ & P09382 & LEG1 & $15 / 18$ & $5.34 / 8.17$ & $16 \%$ & 140 & $\mathrm{~T} 24$ \\
\hline S162 & P44 & P09382 & LEG1 & $15 / 15$ & $5.34 / 5.46$ & $16 \%$ & 100 & $\mathrm{~T} 24$ \\
\hline
\end{tabular}




\begin{tabular}{|c|c|c|c|c|c|c|c|c|}
\hline S163 & P44 & P09382 & LEG1 & $15.05 / 15$ & $5.34 / 5.35$ & $23 \%$ & 230 & SV \\
\hline S164 & P45 & Q12912 & LRMP & $62.7 / 28$ & $5.62 / 5.53$ & $11 \%$ & 60 & T24 \\
\hline S165 & P45 & Q12912 & LRMP & $62.7 / 18$ & $5.62 / 5.45$ & $13 \%$ & 71 & 5637 \\
\hline S166 & P45 & Q12912 & LRMP & $62.75 / 11$ & $5.62 / 6.06$ & $7 \%$ & 70 & SV \\
\hline S167 & P45 & Q12912 & LRMP & $62.75 / 10$ & $5.62 / 3.37$ & $9 \%$ & 87 & SV \\
\hline S168 & P45 & Q12912 & LRMP & $62.75 / 27$ & $5.62 / 7.95$ & $14 \%$ & 79 & SV \\
\hline S169 & P45 & Q12912 & LRMP & $62.75 / 35$ & $5.62 / 3.97$ & $10 \%$ & 64 & SV \\
\hline S170 & P46 & P40925 & MDHC & $36.63 / 37$ & $6.89 / 6.09$ & $13 \%$ & 69 & SV \\
\hline S171 & P47 & P40926 & MDHM & $35.94 / 38$ & $8.54 / 10$ & $17 \%$ & 63 & 5637 \\
\hline S172 & P47 & P40926 & MDHM & $35.94 / 37$ & $8.54 / 10$ & $11 \%$ & 187 & SV \\
\hline S173 & P48 & Q96G25 & MED8 & $29.18 / 12$ & $6.92 / 5.70$ & $7 \%$ & 69 & T24 \\
\hline S174 & P49 & P14174 & MIF & $12.64 / 12$ & $8.24 / 7.95$ & $7 \%$ & 75 & T24 \\
\hline S175 & P49 & P14174 & MIF & $12.64 / 12$ & $8.24 / 9.17$ & $7 \%$ & 64 & SV \\
\hline S176 & P49 & P14174 & MIF & $12.64 / 12$ & $8.24 / 10$ & $9 \%$ & 65 & SV \\
\hline S177 & P50 & P08253 & MMP2 & $72 / 21$ & $5.02 / 4.52$ & $12 \%$ & 60 & 5637 \\
\hline S178 & P51 & P15531 & NDKA & $17.31 / 21$ & $5.83 / 5.83$ & $20 \%$ & 146 & T24 \\
\hline S179 & P51 & P15531 & NDKA & $17.31 / 21$ & $5.83 / 5.64$ & $19 \%$ & 75 & T24 \\
\hline S180 & P51 & P15531 & NDKA & $17.31 / 20$ & $5.83 / 5.83$ & $14 \%$ & 68 & 5637 \\
\hline S181 & P51 & P15531 & NDKA & $17.31 / 20$ & $5.83 / 5.42$ & $20 \%$ & 76 & 5637 \\
\hline S182 & P51 & P15531 & NDKA & $17.31 / 19$ & $5.83 / 6.01$ & $20 \%$ & 75 & 5637 \\
\hline S183 & P51 & P15531 & NDKA & $17.3 / 20$ & $5.83 / 10$ & $20 \%$ & 130 & SV \\
\hline S184 & P51 & P15531 & NDKA & $17.3 / 22$ & $5.83 / 5.93$ & $20 \%$ & 203 & SV \\
\hline S185 & P51 & P15531 & NDKA & $17.3 / 22$ & $5.83 / 5.98$ & $14 \%$ & 71 & SV \\
\hline S186 & P51 & P15531 & NDKA & $17.31 / 24$ & $5.83 / 5.69$ & $14 \%$ & 104 & SV \\
\hline S187 & P52 & P22392 & NDKB & $17.40 / 18$ & $8.52 / 10.00$ & $24 \%$ & 129 & T24 \\
\hline S188 & P52 & P22392 & NDKB & $17.40 / 18$ & $8.52 / 9.58$ & $20 \%$ & 82 & T24 \\
\hline S189 & P52 & P22392 & NDKB & $17.40 / 18$ & $8.52 / 8.46$ & $20 \%$ & 63 & T24 \\
\hline S190 & P52 & P22392 & NDKB & $17.40 / 18$ & $8.52 / 8.52$ & $20 \%$ & 68 & 5637 \\
\hline S191 & P52 & P22392 & NDKB & $17.40 / 18$ & $8.52 / 10$ & $20 \%$ & 97 & 5637 \\
\hline S192 & P52 & P22392 & NDKB & $17.4 / 20$ & $8.52 / 9.08$ & $31 \%$ & 227 & SV \\
\hline S193 & P52 & P22392 & NDKB & $17.4 / 18$ & $8.52 / 10.01$ & $14 \%$ & 85 & SV \\
\hline S194 & P52 & P22392 & NDKB & $17.4 / 18$ & $8.52 / 10$ & $20 \%$ & 161 & SV \\
\hline S195 & P52 & P22392 & NDKB & $17.4 / 17$ & $8.52 / 6.06$ & $14 \%$ & 75 & SV \\
\hline S196 & P52 & P22392 & NDKB & $17.4 / 18$ & $8.52 / 9.78$ & $14 \%$ & 82 & SV \\
\hline S197 & P53 & P80188 & NGAL & $22.47 / 24$ & $9.02 / 4.66$ & $11 \%$ & 69 & 5637 \\
\hline S198 & P53 & P80188 & NGAL & $22.47 / 25$ & $9.02 / 10$ & $15 \%$ & 78 & 5637 \\
\hline S199 & P54 & P46087 & NOP2 & $89.59 / 15$ & $9.27 / 7.39$ & $8 \%$ & 70 & 5637 \\
\hline S200 & P54 & P46087 & NOP2 & $89.59 / 29$ & $9.27 / 5.93$ & $11 \%$ & 62 & 5637 \\
\hline S201 & P54 & P46087 & NOP2 & $89.59 / 30$ & $9.27 / 6.06$ & $21 \%$ & 70 & SV \\
\hline S202 & P54 & P46087 & NOP2 & $89.59 / 41$ & $9.27 / 5.71$ & $13 \%$ & 66 & SV \\
\hline S203 & P54 & P46087 & NOP2 & $89.59 / 19$ & $9.27 / 9.32$ & $19 \%$ & 83 & SV \\
\hline S204 & P54 & P46087 & NOP2 & $89.59 / 16$ & $9.27 / 5.68$ & $9 \%$ & 92 & SV \\
\hline S205 & P54 & P46087 & NOP2 & $89.59 / 16$ & $9.27 / 5.90$ & $18 \%$ & 82 & SV \\
\hline S206 & P54 & P46087 & NOP2 & $89.59 / 11$ & $9.27 / 5.93$ & $12 \%$ & 72 & SV \\
\hline S207 & P55 & P06748 & NPM & $32.73 / 29$ & $4.64 / 4.17$ & $14 \%$ & 97 & SV \\
\hline S208 & P55 & P06748 & NPM & $32.73 / 34$ & $4.64 / 4.96$ & $11 \%$ & 136 & SV \\
\hline S209 & P55 & P06748 & NPM & $32.73 / 21$ & $4.64 / 4.17$ & $7 \%$ & 61 & SV \\
\hline $\mathrm{S} 210$ & P56 & P61970 & NTF2 & $14.64 / 13$ & $5.10 / 5.10$ & $11 \%$ & 83 & SV \\
\hline S211 & P57 & P05121 & PAI1 & $45.09 / 51$ & $6.68 / 7.33$ & $9 \%$ & 67 & $\mathrm{~T} 24$ \\
\hline S212 & P57 & P05121 & PAI1 & $45.09 / 51$ & $6.68 / 7.37$ & $8 \%$ & 84 & T24 \\
\hline S213 & P57 & P05121 & PAI1 & $45.09 / 50$ & $6.68 / 7.45$ & $11 \%$ & 66 & T24 \\
\hline S214 & P57 & P05121 & PAI1 & $45.09 / 49$ & $6.68 / 7.55$ & $4 \%$ & 73 & T24 \\
\hline S215 & P57 & P05121 & PAI1 & $45.09 / 37$ & $6.68 / 7.26$ & $7 \%$ & 84 & T24 \\
\hline S216 & P58 & Q99497 & PARK7 & $20 / 25$ & $6.32 / 6.09$ & $17 \%$ & 76 & SV \\
\hline S217 & P59 & P12004 & PCNA & $29.1 / 34$ & $4.57 / 4.71$ & $9 \%$ & 96 & SV \\
\hline S218 & P60 & P07237 & PDIA1 & $57.48 / 68$ & $4.69 / 5.03$ & $5 \%$ & 86 & 5637 \\
\hline
\end{tabular}




\begin{tabular}{|c|c|c|c|c|c|c|c|c|}
\hline S219 & $\mathrm{P} 60$ & P07237 & PDIA1 & $57.48 / 58$ & $4.69 / 4.96$ & $6 \%$ & 199 & SV \\
\hline S220 & P61 & P30101 & PDIA3 & $57.48 / 56$ & $5.61 / 5.89$ & $7 \%$ & 199 & SV \\
\hline S221 & P62 & P30086 & PEBP1 & $21.16 / 23$ & $7.43 / 9.56$ & $7 \%$ & 61 & SV \\
\hline S222 & P63 & P18669 & PGAM1 & $28.9 / 30$ & $6.75 / 6.99$ & $8 \%$ & 97 & SV \\
\hline S223 & P63 & P18669 & PGAM1 & $28.9 / 30$ & $6.75 / 7.93$ & $16 \%$ & 167 & SV \\
\hline S224 & P63 & P18669 & PGAM1 & $28.9 / 28$ & $6.75 / 8.04$ & $12 \%$ & 191 & SV \\
\hline S225 & P64 & P98160 & PGBM & $47.92 / 25$ & $6.03 / 5.62$ & $12 \%$ & 119 & 5637 \\
\hline S226 & P65 & P00558 & PGK1 & $44.99 / 45$ & $5.99 / 8.18$ & $14 \%$ & 83 & $\mathrm{~T} 24$ \\
\hline S227 & P65 & P00558 & PGK1 & $44.99 / 45$ & $5.99 / 8.83$ & $16 \%$ & 75 & T24 \\
\hline S228 & P65 & P00558 & PGK1 & $44.99 / 45$ & $5.99 / 9.03$ & $11 \%$ & 71 & T24 \\
\hline S229 & P65 & P00558 & PGK1 & $44.98 / 44$ & $8.30 / 9.97$ & $9 \%$ & 141 & SV \\
\hline $\mathrm{S} 230$ & P66 & P61457 & PHS & $12.00 / 12$ & $6.28 / 7.12$ & $24 \%$ & 54 & $\mathrm{~T} 24$ \\
\hline $\mathrm{S} 231$ & P66 & P61457 & PHS & $12.05 / 10$ & $6.28 / 6.22$ & $23 \%$ & 83 & SV \\
\hline $\mathrm{S} 232$ & P67 & Q9UNA4 & POLI & $81.09 / 29$ & $5.73 / 4.79$ & $12 \%$ & 81 & $\mathrm{~T} 24$ \\
\hline S233 & P68 & P27169 & PON1 & $39.89 / 28$ & $5.73 / 7.50$ & $14 \%$ & 136 & $\mathrm{~T} 24$ \\
\hline S234 & P68 & P27169 & PON1 & $39.89 / 30$ & $5.73 / 5.98$ & $13 \%$ & 107 & $\mathrm{~T} 24$ \\
\hline S235 & P68 & P27169 & PON1 & $39.89 / 30$ & $5.73 / 6.06$ & $14 \%$ & 90 & T24 \\
\hline S236 & P69 & P62937 & PPIA & $18.23 / 18$ & $9.32 / 7.66$ & $19 \%$ & 95 & T24 \\
\hline S237 & P69 & P62937 & PPIA & $18.23 / 18$ & $9.32 / 8.63$ & $24 \%$ & 189 & T24 \\
\hline S238 & P69 & P62937 & PPIA & $18.23 / 17$ & $7.66 / 8.02$ & $19 \%$ & 90 & 5637 \\
\hline S239 & P69 & P62937 & PPIA & $18.23 / 17$ & $7.66 / 8.53$ & $28 \%$ & 86 & 5637 \\
\hline S240 & P69 & P62937 & PPIA & $18.23 / 17$ & $7.66 / 6.05$ & $2 \%$ & 84 & 5637 \\
\hline S241 & P69 & P62937 & PPIA & $18.23 / 17$ & $7.66 / 8.40$ & $19 \%$ & 62 & 5637 \\
\hline S242 & P69 & P62937 & PPIA & $18.23 / 17$ & $7.66 / 8.45$ & $12 \%$ & 63 & 5637 \\
\hline S243 & P69 & P62937 & PPIA & $18.23 / 18$ & $7.66 / 7.59$ & $19 \%$ & 67 & SV \\
\hline S244 & P69 & P62937 & PPIA & $18.23 / 18$ & $7.66 / 8.94$ & $19 \%$ & 152 & SV \\
\hline S245 & P69 & P62937 & PPIA & $18.23 / 17$ & $7.66 / 9.44$ & $17 \%$ & 69 & SV \\
\hline S246 & P69 & P62937 & PPIA & $18.23 / 17$ & $7.66 / 9.89$ & $19 \%$ & 189 & SV \\
\hline S247 & P70 & Q06830 & PRDX1 & $22.32 / 26$ & $8.3 / 7.48$ & $16 \%$ & 147 & T24 \\
\hline S248 & P70 & Q06830 & PRDX1 & $22.32 / 25$ & $8.3 / 7.48$ & $16 \%$ & 89 & T24 \\
\hline S249 & P70 & Q06830 & PRDX1 & $22.32 / 26$ & $8.3 / 8.35$ & $14 \%$ & 89 & $\mathrm{~T} 24$ \\
\hline $\mathrm{S} 250$ & P70 & Q06830 & PRDX1 & $22.32 / 26$ & $8.3 / 6.53$ & $10 \%$ & 64 & T24 \\
\hline S251 & P70 & Q06830 & PRDX1 & $22.32 / 25$ & $8.3 / 7.14$ & $10 \%$ & 64 & T24 \\
\hline $\mathrm{S} 252$ & P70 & Q06830 & PRDX1 & $22.32 / 26$ & $8.3 / 7.15$ & $14 \%$ & 80 & $\mathrm{~T} 24$ \\
\hline S253 & P70 & Q06830 & PRDX1 & $22.32 / 25$ & $8.3 / 6.06$ & $20 \%$ & 93 & 5637 \\
\hline S254 & P70 & Q06830 & PRDX1 & $22.32 / 25$ & $8.3 / 6.06$ & $20 \%$ & 108 & 5637 \\
\hline S255 & P70 & Q06830 & PRDX1 & $22.32 / 25$ & $8.3 / 8.3$ & $20 \%$ & 81 & 5637 \\
\hline S256 & P70 & Q06830 & PRDX1 & $22.32 / 25$ & $8.3 / 8.3$ & $10 \%$ & 80 & 5637 \\
\hline S257 & P70 & Q06830 & PRDX1 & $22.32 / 28$ & $8.3 / 8.21$ & $14 \%$ & 78 & SV \\
\hline S258 & P70 & Q06830 & PRDX1 & $22.32 / 25$ & $8.3 / 9.62$ & $10 \%$ & 71 & SV \\
\hline S259 & P70 & Q06830 & PRDX1 & $22.32 / 26$ & $8.3 / 10.03$ & $10 \%$ & 67 & SV \\
\hline S260 & P71 & P32119 & PRDX2 & $22.05 / 23$ & $5.67 / 5.42$ & $14 \%$ & 120 & 5637 \\
\hline S261 & P72 & P30048 & PRDX3 & $28.02 / 26$ & $5.77 / 6.10$ & $14 \%$ & 199 & SV \\
\hline S262 & P73 & P30041 & PRDX6 & $25.13 / 29$ & $6.00 / 6.15$ & $9 \%$ & 69 & SV \\
\hline S263 & P74 & P07737 & PROF1 & $15.22 / 15$ & $8.47 / 7.93$ & $32 \%$ & 143 & T24 \\
\hline S264 & P74 & P07737 & PROF1 & $15.22 / 14$ & $8.47 / 7.95$ & $32 \%$ & 206 & $\mathrm{~T} 24$ \\
\hline S265 & P74 & P07737 & PROF1 & $15.22 / 15$ & $8.47 / 10.07$ & $23 \%$ & 206 & SV \\
\hline S266 & P74 & P07737 & PROF1 & $15.22 / 15$ & $8.47 / 9.32$ & $10 \%$ & 96 & SV \\
\hline S267 & P75 & P25787 & PSA2 & $26 / 27$ & $6.92 / 6.42$ & $13 \%$ & 110 & SV \\
\hline S268 & P75 & P25787 & PSA2 & $26 / 27$ & $6.92 / 8.21$ & $11 \%$ & 29 & SV \\
\hline S269 & P76 & P28066 & PSA5 & $26.56 / 30$ & $4.74 / 4.77$ & $12 \%$ & 98 & SV \\
\hline S270 & P77 & P60900 & PSA6 & $27.84 / 31$ & $6.34 / 7.14$ & $9 \%$ & 85 & $\mathrm{~T} 24$ \\
\hline S271 & P78 & P49721 & PSB2 & $22.99 / 25$ & $6.52 / 7.55$ & $15 \%$ & 80 & SV \\
\hline S272 & P79 & P49720 & PSB3 & $23.22 / 27$ & $6.12 / 6.09$ & $8 \%$ & 69 & SV \\
\hline S273 & P80 & P28070 & PSB4 & $29.24 / 27$ & $5.44 / 5.78$ & $8 \%$ & 135 & SV \\
\hline S274 & P81 & P28074 & PSB5 & $28.63 / 28$ & $8.66 / 10.07$ & $5 \%$ & 61 & SV \\
\hline
\end{tabular}




\begin{tabular}{|c|c|c|c|c|c|c|c|c|}
\hline S275 & P82 & P28072 & PSB6 & $25.57 / 25$ & $4.91 / 5.07$ & $8 \%$ & 63 & SV \\
\hline S276 & P83 & Q99436 & PSB7 & $30.29 / 30$ & $5.61 / 5.94$ & $23 \%$ & 166 & SV \\
\hline S277 & P84 & Q15223 & PVRL1 & $54.06 / 18$ & $5.54 / 8.41$ & $13 \%$ & 92 & 5637 \\
\hline S278 & P85 & Q32P51 & RA1L2 & $34.38 / 24$ & $9.08 / 7.55$ & $16 \%$ & 90 & $\mathrm{~T} 24$ \\
\hline S279 & P85 & Q32P51 & RA1L2 & $34.37 / 25$ & $9.08 / 9.29$ & $7 \%$ & 93 & $\mathrm{SV}$ \\
\hline S280 & P85 & Q32P51 & RA1L2 & $34.37 / 25$ & 9.08/9.91 & $6 \%$ & 62 & SV \\
\hline S281 & P85 & Q32P51 & RA1L2 & $34.37 / 24$ & $9.08 / 8.61$ & $7 \%$ & 62 & SV \\
\hline S282 & P86 & P22626 & ROA2 & $37.46 / 35$ & $8.97 / 7.50$ & $7 \%$ & 73 & 5637 \\
\hline S283 & P86 & P22626 & ROA2 & $37.46 / 35$ & $8.97 / 8.36$ & $8 \%$ & 69 & 5637 \\
\hline S284 & P86 & P22626 & ROA2 & $37.46 / 38$ & $8.97 / 10.07$ & $14 \%$ & 104 & SV \\
\hline S285 & P87 & P62306 & RUXF & $9.776 / 10$ & $4.63 / 5.04$ & $9 \%$ & 74 & SV \\
\hline S286 & P88 & O00560 & SDCB1 & $32.59 / 35$ & $7.05 / 7.56$ & $7 \%$ & 95 & $\mathrm{~T} 24$ \\
\hline S287 & P89 & Q01105 & SET & $33.47 / 42$ & $4.22 / 5.18$ & $10 \%$ & 88 & SV \\
\hline S288 & P90 & P00441 & SODC & $16.15 / 19$ & $5.70 / 5.61$ & $13 \%$ & 87 & 5637 \\
\hline S289 & P91 & P09486 & SPARC & $35.46 / 41$ & $4.73 / 4.73$ & $23 \%$ & 392 & SV \\
\hline S290 & P91 & P09486 & SPARC & $35.46 / 40$ & $4.73 / 4.08$ & $15 \%$ & 242 & SV \\
\hline S291 & P91 & P09486 & SPARC & $35.46 / 47$ & $4.73 / 10$ & $15 \%$ & 163 & SV \\
\hline S294 & P91 & P09486 & SPARC & $35.47 / 43$ & $4.73 / 4.46$ & $19 \%$ & 234 & T24 \\
\hline S292 & P92 & P36952 & SPB5 & $42.53 / 42$ & $5.72 / 5.95$ & $14 \%$ & 270 & SV \\
\hline S293 & P92 & P36952 & SPB5 & $42.53 / 41$ & $5.72 / 5.81$ & $7 \%$ & 73 & SV \\
\hline S295 & P93 & Q04837 & SSBP & $17.25 / 16$ & $8.24 / 10.01$ & $11 \%$ & 77 & SV \\
\hline S296 & P94 & P40763 & STAT3 & $88.81 / 13$ & $5.94 / 3.68$ & $12 \%$ & 91 & 5637 \\
\hline S297 & P95 & P37802 & TAGL2 & $22.55 / 24$ & $8.24 / 10.07$ & $10 \%$ & 98 & SV \\
\hline S298 & P96 & Q9NU19 & TB22B & $59.33 / 69$ & $6.94 / 4.71$ & $12 \%$ & 94 & 5637 \\
\hline S299 & P97 & Q13885 & TBB2A & $50.27 / 59$ & $4.78 / 4.85$ & $10 \%$ & 114 & 5637 \\
\hline S300 & P97 & Q13885 & TBB2A & $50.27 / 52$ & $4.78 / 5.21$ & $14 \%$ & 139 & SV \\
\hline S301 & P98 & P13693 & ТСТР & $19.7 / 26$ & $4.84 / 4.53$ & $12 \%$ & 95 & 5637 \\
\hline S302 & P98 & P13693 & ТСТР & $19.7 / 26$ & $4.84 / 4.65$ & $9 \%$ & 87 & 5637 \\
\hline S303 & P98 & P13693 & ТСТР & $19.697 / 25$ & $4.84 / 4.99$ & $20 \%$ & 150 & SV \\
\hline S304 & P98 & P13693 & ТCTP & $19.697 / 26$ & $4.84 / 4.98$ & $15 \%$ & 85 & SV \\
\hline S305 & P99 & Q9P273 & TEN3 & $30.5 / 40$ & $5.99 / 9.69$ & $10 \%$ & 89 & $\mathrm{~T} 24$ \\
\hline S306 & P100 & P01033 & TIMP1 & $23.84 / 33$ & $8.46 / 7.37$ & $18 \%$ & 103 & T24 \\
\hline S307 & P100 & P01033 & TIMP1 & $23.84 / 34$ & $8.46 / 7.15$ & $11 \%$ & 192 & T24 \\
\hline S308 & P100 & P01033 & TIMP1 & $23.84 / 33$ & $8.46 / 7.16$ & $13 \%$ & 137 & T24 \\
\hline S309 & P100 & P01033 & TIMP1 & $23.84 / 33$ & $8.46 / 7.23$ & $17 \%$ & 153 & $\mathrm{~T} 24$ \\
\hline S310 & P100 & P01033 & TIMP1 & $23.84 / 34$ & $8.46 / 7.46$ & $11 \%$ & 169 & T24 \\
\hline S311 & P100 & P01033 & TIMP1 & $23.84 / 33$ & $8.46 / 7.46$ & $14 \%$ & 182 & T24 \\
\hline S312 & P100 & P01033 & TIMP1 & $23.84 / 33$ & $8.46 / 7.51$ & $19 \%$ & 140 & T24 \\
\hline S313 & P100 & P01033 & TIMP1 & $23.84 / 33$ & $8.46 / 8.18$ & $12 \%$ & 153 & $\mathrm{~T} 24$ \\
\hline S314 & P100 & P01033 & TIMP1 & $23.84 / 32$ & $8.46 / 8.14$ & $11 \%$ & 110 & T24 \\
\hline S315 & P100 & P01033 & TIMP1 & $23.84 / 31$ & $8.46 / 8.21$ & $14 \%$ & 148 & T24 \\
\hline S316 & P101 & P16035 & TIMP2 & $25.07 / 45$ & $8.46 / 8.62$ & $12 \%$ & 61 & T24 \\
\hline S317 & P101 & P16035 & TIMP2 & $25.07 / 24$ & $7.45 / 7.64$ & $10 \%$ & 65 & T24 \\
\hline S318 & P101 & P16035 & TIMP2 & $25.07 / 26$ & $7.45 / 6.85$ & $10 \%$ & 105 & T24 \\
\hline S319 & P101 & P16035 & TIMP2 & $25.07 / 26$ & 7.45/7.06 & $14 \%$ & 68 & T24 \\
\hline S320 & P101 & P16035 & TIMP2 & $25.07 / 26$ & $7.45 / 5.89$ & $9 \%$ & 70 & $\mathrm{~T} 24$ \\
\hline S321 & P101 & P16035 & TIMP2 & $25.07 / 26$ & $7.45 / 5.99$ & $10 \%$ & 96 & T24 \\
\hline S322 & P101 & P16035 & TIMP2 & $25.07 / 26$ & $7.45 / 5.89$ & $10 \%$ & 93 & T24 \\
\hline S323 & P101 & P16035 & TIMP2 & $25.07 / 25$ & $7.45 / 5.98$ & $10 \%$ & 82 & $\mathrm{~T} 24$ \\
\hline S324 & P102 & Q9GZM7 & TINAL & $50.15 / 17$ & $6.42 / 6.21$ & $13 \%$ & 74 & 5637 \\
\hline S325 & P103 & P60174 & TPIS & $26.94 / 30$ & $6.45 / 7.49$ & $32 \%$ & 246 & T24 \\
\hline S326 & P103 & P60174 & TPIS & $26.94 / 30$ & $6.45 / 7.24$ & $27 \%$ & 284 & T24 \\
\hline S327 & P103 & P60174 & TPIS & $26.94 / 30$ & $6.45 / 6.63$ & $13 \%$ & 127 & $\mathrm{~T} 24$ \\
\hline S328 & P103 & P60174 & TPIS & $26.94 / 29$ & $6.45 / 8.33$ & $9 \%$ & 441 & 5637 \\
\hline S329 & P103 & P60174 & TPIS & $26.94 / 27$ & $6.45 / 5.61$ & $8 \%$ & 90 & SV \\
\hline S330 & P103 & P60174 & TPIS & $26.94 / 28$ & $6.45 / 6.66$ & $16 \%$ & 149 & SV \\
\hline
\end{tabular}




\begin{tabular}{lllcccccc} 
S331 & P103 & P60174 & TPIS & $26.94 / 28$ & $6.45 / 6.09$ & $18 \%$ & 96 & SV \\
S332 & P104 & P06753 & TPM3 & $32.86 / 34$ & $4.68 / 5.18$ & $8 \%$ & 111 & SV \\
S333 & P105 & P07996 & TSP1 & $133.3 / 29$ & $4.70 / 8.31$ & $13 \%$ & 239 & 5637 \\
S334 & P105 & P07996 & TSP1 & $133.3 / 28$ & $4.70 / 8.31$ & $14 \%$ & 228 & 5637 \\
S335 & P105 & P07996 & TSP1 & $133.3 / 28$ & $4.70 / 8.42$ & $13 \%$ & 91 & 5637 \\
S336 & P105 & P07996 & TSP1 & $133.3 / 43$ & $4.70 / 4.88$ & $14 \%$ & 124 & 5637 \\
S337 & P105 & P07996 & TSP1 & $133.3 / 29$ & $4.70 / 6.05$ & $16 \%$ & 200 & 5637 \\
S338 & P105 & P07996 & TSP1 & $133.3 / 29$ & $4.70 / 6.51$ & $19 \%$ & 113 & 5637 \\
S339 & P106 & Q8N2E2 & VWDE & $183.3 / 13$ & $5.45 / 4.34$ & $8 \%$ & 95 & 5637 \\
S340 & P107 & O00755 & WNT7A & $40.41 / 53$ & $8.83 / 7.55$ & $14 \%$ & 105 & T24 \\
S341 & P108 & Q9UJN7 & ZN391 & $41.83 / 32$ & $8.94 / 8.49$ & $9 \%$ & 126 & SV \\
\hline
\end{tabular}


Table S3. Secretory characteristic analysis of the 108 proteins.

\begin{tabular}{|c|c|c|c|c|c|c|}
\hline NO. & $\begin{array}{c}\text { Swissprot } \\
\text { number }\end{array}$ & $\begin{array}{c}\text { Protein } \\
\text { name }\end{array}$ & $\begin{array}{l}\text { classical } \\
\text { secreted } \\
\text { proteins }\end{array}$ & $\begin{array}{c}\text { non- } \\
\text { classical } \\
\text { secreted } \\
\text { proteins }\end{array}$ & $\begin{array}{l}\text { urinary } \\
\text { proteins }\end{array}$ & $\begin{array}{l}\text { Single spots or } \\
\text { multiple spots } \\
\text { (with_Ychanged } \\
\text { MW or with_N } \\
\text { no changed } \\
\text { MW) }\end{array}$ \\
\hline P1 & P31947 & $1433 \mathrm{~S}$ & $\mathrm{NO}$ & $\mathrm{NO}$ & $\mathrm{NO}$ & single \\
\hline $\mathrm{P} 2$ & O95336 & 6PGL & $\mathrm{NO}$ & YES & YES & single \\
\hline P3 & P05067 & A4 & YES & NO & YES & single \\
\hline P4 & P62736 & ACTA & $\mathrm{NO}$ & $\mathrm{NO}$ & $\mathrm{NO}$ & multiple_N \\
\hline P5 & P60709 & ACTB & $\mathrm{NO}$ & $\mathrm{NO}$ & YES & multiple_N \\
\hline P6 & O00468 & AGRIN & YES & $\mathrm{NO}$ & YES & single \\
\hline P7 & P02768 & ALBU & YES & $\mathrm{NO}$ & YES & multiple_N \\
\hline P8 & P05091 & ALDH2 & $\mathrm{NO}$ & YES & $\mathrm{NO}$ & single \\
\hline P9 & P04075 & ALDOA & $\mathrm{NO}$ & NO & YES & multiple_N \\
\hline P10 & P15121 & ALDR & $\mathrm{NO}$ & $\mathrm{NO}$ & YES & multiple_Y \\
\hline P11 & P39687 & AN32A & $\mathrm{NO}$ & $\mathrm{NO}$ & YES & single \\
\hline P12 & P01008 & ANT3 & YES & $\mathrm{NO}$ & YES & single \\
\hline P13 & P02649 & APOE & YES & $\mathrm{NO}$ & YES & single \\
\hline P14 & P05089 & ARGI1 & $\mathrm{NO}$ & $\mathrm{NO}$ & YES & multiple_N \\
\hline P15 & P61769 & B2MG & YES & $\mathrm{NO}$ & YES & multiple_Y \\
\hline P16 & P27797 & CALR & YES & $\mathrm{NO}$ & YES & multiple_N \\
\hline P17 & P04040 & CATA & $\mathrm{NO}$ & $\mathrm{NO}$ & YES & single \\
\hline P18 & P07339 & CATD & YES & $\mathrm{NO}$ & YES & multiple_N \\
\hline P19 & P10809 & CH60 & NO & $\mathrm{NO}$ & YES & single \\
\hline P20 & P23528 & COF1 & $\mathrm{NO}$ & YES & $\mathrm{NO}$ & single \\
\hline $\mathrm{P} 21$ & P00167 & CYB5 & $\mathrm{NO}$ & $\mathrm{NO}$ & YES & single \\
\hline $\mathrm{P} 22$ & P01034 & CYTC & YES & $\mathrm{NO}$ & YES & single \\
\hline $\mathrm{P} 23$ & Q30KQ2 & DB130 & YES & $\mathrm{NO}$ & $\mathrm{NO}$ & single \\
\hline $\mathrm{P} 24$ & Q15392 & DHC24 & YES & $\mathrm{NO}$ & $\mathrm{NO}$ & single \\
\hline $\mathrm{P} 25$ & Q18PE1 & DOK7 & NO & $\mathrm{NO}$ & $\mathrm{NO}$ & multiple_N \\
\hline $\mathrm{P} 26$ & P06733 & ENOA & $\mathrm{NO}$ & $\mathrm{NO}$ & YES & multiple_N \\
\hline $\mathrm{P} 27$ & P16930 & FAAA & NO & $\mathrm{NO}$ & YES & single \\
\hline P28 & P02751 & FINC & YES & $\mathrm{NO}$ & YES & multiple_N \\
\hline P29 & Q13283 & G3BP1 & $\mathrm{NO}$ & $\mathrm{NO}$ & YES & single \\
\hline P30 & P04406 & G3P & $\mathrm{NO}$ & $\mathrm{NO}$ & YES & multiple_Y \\
\hline P31 & P78417 & GSTO1 & $\mathrm{NO}$ & $\mathrm{NO}$ & YES & single \\
\hline P32 & P09211 & GSTP1 & $\mathrm{NO}$ & $\mathrm{NO}$ & YES & multiple_N \\
\hline P33 & Q96QV6 & H2A1A & $\mathrm{NO}$ & $\mathrm{NO}$ & $\mathrm{NO}$ & single \\
\hline P34 & Q96A08 & H2B1A & $\mathrm{NO}$ & $\mathrm{NO}$ & $\mathrm{NO}$ & single \\
\hline P35 & P33778 & H2B1B & $\mathrm{NO}$ & $\mathrm{NO}$ & $\mathrm{NO}$ & single \\
\hline P36 & P62805 & $\mathrm{H} 4$ & $\mathrm{NO}$ & $\mathrm{NO}$ & YES & single \\
\hline P37 & P68871 & $\mathrm{HBB}$ & $\mathrm{NO}$ & $\mathrm{NO}$ & YES & single \\
\hline P38 & P04792 & HSPB1 & $\mathrm{NO}$ & YES & YES & multiple_Y \\
\hline P39 & P12277 & KCRB & $\mathrm{NO}$ & $\mathrm{NO}$ & YES & multiple_Y \\
\hline P40 & P06732 & KCRM & $\mathrm{NO}$ & $\mathrm{NO}$ & $\mathrm{NO}$ & single \\
\hline $\mathrm{P} 41$ & Q92876 & KLK6 & YES & $\mathrm{NO}$ & YES & multiple_Y \\
\hline $\mathrm{P} 42$ & P14618 & KPYM & $\mathrm{NO}$ & $\mathrm{NO}$ & YES & single \\
\hline P43 & P11047 & LAMC1 & YES & $\mathrm{NO}$ & YES & single \\
\hline P44 & P09382 & LEG1 & $\mathrm{NO}$ & $\mathrm{NO}$ & YES & multiple_N \\
\hline P45 & Q12912 & LRMP & $\mathrm{NO}$ & YES & $\mathrm{NO}$ & multiple_N \\
\hline P46 & P40925 & MDHC & $\mathrm{NO}$ & $\mathrm{NO}$ & YES & single \\
\hline P47 & P40926 & MDHM & $\mathrm{NO}$ & YES & YES & multiple_Y \\
\hline
\end{tabular}




\begin{tabular}{|c|c|c|c|c|c|c|}
\hline P48 & Q96G25 & MED8 & $\mathrm{NO}$ & NO & $\mathrm{NO}$ & single \\
\hline P49 & P14174 & MIF & NO & YES & YES & single \\
\hline P50 & P08253 & MMP2 & YES & NO & YES & single \\
\hline P51 & P15531 & NDKA & NO & NO & $\mathrm{NO}$ & multiple \\
\hline P52 & P22392 & NDKB & NO & NO & YES & multiple_N \\
\hline P53 & P80188 & NGAL & YES & NO & YES & multiple_Y \\
\hline P54 & P46087 & NOP2 & $\mathrm{NO}$ & YES & $\mathrm{NO}$ & multiple_N \\
\hline P55 & P06748 & NPM & NO & YES & $\mathrm{NO}$ & multiple_N \\
\hline P56 & P61970 & NTF2 & NO & YES & YES & single \\
\hline P57 & P05121 & PAI1 & YES & NO & YES & multiple_Y \\
\hline P58 & Q99497 & PARK7 & $\mathrm{NO}$ & NO & YES & single \\
\hline P59 & P12004 & PCNA & NO & NO & $\mathrm{NO}$ & single \\
\hline P60 & P07237 & PDIA1 & YES & NO & YES & single \\
\hline P61 & P30101 & PDIA3 & YES & NO & YES & single \\
\hline P62 & P30086 & PEBP1 & $\mathrm{NO}$ & YES & YES & single \\
\hline P63 & P18669 & PGAM1 & NO & NO & YES & multiple_Y \\
\hline P64 & P98160 & PGBM & YES & NO & YES & single \\
\hline P65 & P00558 & PGK1 & NO & NO & YES & multiple_Y \\
\hline P66 & P61457 & PHS & NO & NO & YES & single \\
\hline P67 & Q9UNA4 & POLI & NO & NO & $\mathrm{NO}$ & single \\
\hline P68 & P27169 & PON1 & YES & NO & YES & multiple $\mathrm{Y}$ \\
\hline P69 & P62937 & PPIA & NO & NO & YES & multiple_Y \\
\hline P70 & Q06830 & PRDX1 & NO & NO & YES & multiple_Y \\
\hline P71 & P32119 & PRDX2 & NO & NO & YES & single \\
\hline P72 & P30048 & PRDX3 & NO & YES & YES & single \\
\hline P73 & P30041 & PRDX6 & NO & NO & YES & single \\
\hline P74 & P07737 & PROF1 & NO & NO & YES & multiple_Y \\
\hline P75 & P25787 & PSA2 & NO & NO & YES & multiple_Y \\
\hline P76 & P28066 & PSA5 & NO & NO & YES & single \\
\hline P77 & P60900 & PSA6 & NO & NO & YES & single \\
\hline P78 & P49721 & PSB2 & NO & NO & YES & single \\
\hline P79 & P49720 & PSB3 & NO & NO & YES & single \\
\hline P80 & P28070 & PSB4 & NO & YES & YES & single \\
\hline P81 & P28074 & PSB5 & NO & YES & YES & single \\
\hline P82 & P28072 & PSB6 & NO & NO & YES & single \\
\hline P83 & Q99436 & PSB7 & NO & NO & YES & single \\
\hline P84 & Q15223 & PVRL1 & YES & NO & YES & single \\
\hline P85 & Q32P51 & RA1L2 & $\mathrm{NO}$ & NO & $\mathrm{NO}$ & multiple_N \\
\hline P86 & P22626 & ROA2 & NO & NO & YES & multiple_N \\
\hline P87 & P62306 & RUXF & NO & NO & NO & single \\
\hline P88 & O00560 & SDCB1 & NO & NO & YES & single \\
\hline P89 & Q01105 & SET & NO & NO & $\mathrm{NO}$ & single \\
\hline P90 & P00441 & SODC & NO & YES & YES & single \\
\hline P91 & P09486 & SPARC & YES & NO & YES & multiple $\mathrm{N}$ \\
\hline P92 & P36952 & SPB5 & $\mathrm{NO}$ & $\mathrm{NO}$ & YES & multiple_Y \\
\hline P93 & Q04837 & SSBP & NO & YES & $\mathrm{NO}$ & single \\
\hline P94 & P40763 & STAT3 & NO & NO & YES & single \\
\hline P95 & P37802 & TAGL2 & NO & YES & YES & single \\
\hline P96 & Q9NU19 & TB22B & NO & $\mathrm{NO}$ & $\mathrm{NO}$ & single \\
\hline P97 & Q13885 & TBB2A & NO & NO & $\mathrm{NO}$ & single \\
\hline P98 & P13693 & ТCTP & NO & $\mathrm{NO}$ & YES & multiple_Y \\
\hline P99 & Q9P273 & TEN3 & NO & NO & $\mathrm{NO}$ & single \\
\hline P100 & P01033 & TIMP1 & YES & NO & YES & multiple_Y \\
\hline P101 & P16035 & TIMP2 & YES & NO & YES & multiple_N \\
\hline P102 & Q9GZM7 & TINAL & YES & NO & YES & single \\
\hline P103 & P60174 & TPIS & NO & NO & YES & multiple_Y \\
\hline
\end{tabular}




\begin{tabular}{lllllll} 
P104 & P06753 & TPM3 & NO & NO & YES & single \\
P105 & P07996 & TSP1 & YES & NO & YES & multiple_N \\
P106 & Q8N2E2 & VWDE & YES & NO & NO & single \\
P107 & O00755 & WNT7A & YES & NO & NO & single \\
P108 & Q9UJN7 & ZN391 & NO & YES & NO & single \\
\hline
\end{tabular}


Table S4. 103 differential proteins from 5637 and T24 vs. SV-HUC-1 by 2DE.

\begin{tabular}{|c|c|c|c|c|c|c|}
\hline NO. & $\begin{array}{c}\text { Swissprot } \\
\text { number }\end{array}$ & $\begin{array}{l}\text { Protein } \\
\text { Name }\end{array}$ & $\begin{array}{l}\text { different in } \\
5637 \text { vs. SV }\end{array}$ & $\begin{array}{l}\text { Fold changes } \\
\text { of } 5637 \text { vs. SV }\end{array}$ & $\begin{array}{l}\text { different in } \\
\text { T24 vs. SV }\end{array}$ & $\begin{array}{l}\text { Fold changes } \\
\text { of T24 vs. SV }\end{array}$ \\
\hline 1 & P62736 & ACTA & YES & 5.894094101 & YES & 1.956810775 \\
\hline 2 & P02768 & ALBU & YES & 13.59693941 & YES & only exits in SV \\
\hline 3 & P07339 & CATD & YES & 0.088645594 & YES & only exits in SV \\
\hline 4 & P46087 & NOP2 & YES & 0.275899741 & YES & only exits in SV \\
\hline 5 & P22626 & ROA2 & YES & 3.04872316 & YES & only exits in SV \\
\hline 6 & Q13885 & TBB2A & YES & 0.17182502 & YES & only exits in SV \\
\hline 7 & Q18PE1 & DOK7 & YES & only exits in 5637 & YES & only exits in T24 \\
\hline 8 & P31947 & $1433 \mathrm{~S}$ & YES & only exits in SV & YES & only exits in SV \\
\hline 9 & O95336 & 6PGL & YES & only exits in SV & YES & only exits in SV \\
\hline 10 & P05091 & ALDH2 & YES & only exits in SV & YES & only exits in SV \\
\hline 11 & P15121 & ALDR & YES & only exits in SV & YES & only exits in SV \\
\hline 12 & P39687 & AN32A & YES & only exits in SV & YES & only exits in SV \\
\hline 13 & P05089 & ARGI1 & YES & only exits in SV & YES & only exits in SV \\
\hline 14 & P10809 & $\mathrm{CH} 60$ & YES & only exits in SV & YES & only exits in SV \\
\hline 15 & P00167 & CYB5 & YES & only exits in SV & YES & only exits in SV \\
\hline 16 & P16930 & FAAA & YES & only exits in SV & YES & only exits in SV \\
\hline 17 & Q13283 & G3BP1 & YES & only exits in SV & YES & only exits in SV \\
\hline 18 & Q96QV6 & $\mathrm{H} 2 \mathrm{~A} 1 \mathrm{~A}$ & YES & only exits in SV & YES & only exits in SV \\
\hline 19 & Q96A08 & H2B1A & YES & only exits in SV & YES & only exits in SV \\
\hline 20 & P62805 & $\mathrm{H} 4$ & YES & only exits in SV & YES & only exits in SV \\
\hline 21 & P04792 & HSPB1 & YES & only exits in SV & YES & only exits in SV \\
\hline 22 & P14618 & KPYM & YES & only exits in SV & YES & only exits in SV \\
\hline 23 & P40925 & MDHC & YES & only exits in SV & YES & only exits in SV \\
\hline 24 & P14174 & MIF & YES & only exits in SV & YES & 0.07735687 \\
\hline 25 & P06748 & NPM & YES & only exits in SV & YES & only exits in SV \\
\hline 26 & P61970 & NTF2 & YES & only exits in SV & YES & only exits in SV \\
\hline 27 & Q99497 & PARK7 & YES & only exits in SV & YES & only exits in SV \\
\hline 28 & P12004 & PCNA & YES & only exits in SV & YES & only exits in SV \\
\hline 29 & P30101 & PDIA3 & YES & only exits in SV & YES & only exits in SV \\
\hline 30 & P30086 & PEBP1 & YES & only exits in SV & YES & only exits in SV \\
\hline 31 & P18669 & PGAM1 & YES & only exits in SV & YES & only exits in SV \\
\hline 32 & P30048 & PRDX3 & YES & only exits in SV & YES & only exits in SV \\
\hline 33 & P30041 & PRDX6 & YES & only exits in SV & YES & only exits in SV \\
\hline 34 & P28066 & PSA5 & YES & only exits in SV & YES & only exits in SV \\
\hline 35 & P49721 & PSB2 & YES & only exits in SV & YES & only exits in SV \\
\hline 36 & P49720 & PSB3 & YES & only exits in SV & YES & only exits in SV \\
\hline 37 & P28070 & PSB4 & YES & only exits in SV & YES & only exits in SV \\
\hline 38 & P28074 & PSB5 & YES & only exits in SV & YES & only exits in SV \\
\hline 39 & P28072 & PSB6 & YES & only exits in SV & YES & only exits in SV \\
\hline 40 & Q99436 & PSB7 & YES & only exits in SV & YES & only exits in SV \\
\hline 41 & Q32P51 & RA1L2 & YES & only exits in SV & YES & 0.104151141 \\
\hline 42 & P62306 & RUXF & YES & only exits in SV & YES & only exits in SV \\
\hline 43 & Q01105 & SET & YES & only exits in SV & YES & only exits in SV \\
\hline 44 & P09486 & SPARC & YES & only exits in SV & YES & 0.061631117 \\
\hline 45 & P36952 & SPB5 & YES & only exits in SV & YES & only exits in SV \\
\hline 46 & Q04837 & SSBP & YES & only exits in SV & YES & only exits in SV \\
\hline 47 & P06753 & TPM3 & YES & only exits in SV & YES & only exits in SV \\
\hline 48 & Q9UJN7 & ZN391 & YES & only exits in SV & YES & only exits in SV \\
\hline 49 & P61769 & $\mathrm{B} 2 \mathrm{MG}$ & YES & 3.137130656 & NO & no significant \\
\hline 50 & P23528 & COF1 & YES & 0.214027903 & $\mathrm{NO}$ & no significant \\
\hline 51 & P02751 & FINC & YES & 9.888183929 & $\mathrm{NO}$ & no significant \\
\hline
\end{tabular}




\begin{tabular}{|c|c|c|c|c|c|c|}
\hline 52 & P40926 & MDHM & YES & 0.084224949 & NO & no significant \\
\hline 53 & P60174 & TPIS & YES & 0.12289571 & $\mathrm{NO}$ & no significant \\
\hline 54 & Q30KQ2 & DB130 & YES & only exits in 5637 & NO & no significant \\
\hline 55 & P33778 & H2B1B & YES & only exits in 5637 & NO & no significant \\
\hline 56 & P11047 & LAMC1 & YES & only exits in 5637 & NO & no significant \\
\hline 57 & P08253 & MMP2 & YES & only exits in 5637 & NO & no significant \\
\hline 58 & P80188 & NGAL & YES & only exits in 5637 & NO & no significant \\
\hline 59 & P98160 & PGBM & YES & only exits in 5637 & $\mathrm{NO}$ & no significant \\
\hline 60 & P32119 & PRDX2 & YES & only exits in 5637 & NO & no significant \\
\hline 61 & Q15223 & PVRL1 & YES & only exits in 5637 & NO & no significant \\
\hline 62 & P00441 & SODC & YES & only exits in 5637 & NO & no significant \\
\hline 63 & P40763 & STAT3 & YES & only exits in 5637 & NO & no significant \\
\hline 64 & Q9NU19 & TB22B & YES & only exits in 5637 & NO & no significant \\
\hline 65 & Q9GZM7 & TINAL & YES & only exits in 5637 & NO & no significant \\
\hline 66 & P07996 & TSP1 & YES & only exits in 5637 & NO & no significant \\
\hline 67 & Q8N2E2 & VWDE & YES & only exits in 5637 & $\mathrm{NO}$ & no significant \\
\hline 68 & P04075 & ALDOA & YES & only exits in SV & NO & no significant \\
\hline 69 & Q92876 & KLK6 & YES & only exits in SV & $\mathrm{NO}$ & no significant \\
\hline 70 & P00558 & PGK1 & YES & only exits in SV & NO & no significant \\
\hline 71 & P61457 & PHS & YES & only exits in SV & $\mathrm{NO}$ & no significant \\
\hline 72 & P07737 & PROF1 & YES & only exits in SV & NO & no significant \\
\hline 73 & P06733 & ENOA & NO & no significant & YES & 3.735575911 \\
\hline 74 & P09211 & GSTP1 & NO & no significant & YES & 0.165607852 \\
\hline 75 & P09382 & LEG1 & NO & no significant & YES & 16.27889 \\
\hline 76 & Q12912 & LRMP & NO & no significant & YES & 0.129271193 \\
\hline 77 & P15531 & NDKA & NO & no significant & YES & 0.221104502 \\
\hline 78 & P05067 & A4 & NO & no significant & YES & only exits in T24 \\
\hline 79 & O00468 & AGRIN & NO & no significant & YES & only exits in T24 \\
\hline 80 & P01008 & ANT3 & NO & no significant & YES & only exits in T24 \\
\hline 81 & Р02649 & APOE & NO & no significant & YES & only exits in T24 \\
\hline 82 & P01034 & CYTC & $\mathrm{NO}$ & no significant & YES & only exits in T24 \\
\hline 83 & Q15392 & DHC24 & $\mathrm{NO}$ & no significant & YES & only exits in T24 \\
\hline 84 & P04406 & G3P & $\mathrm{NO}$ & no significant & YES & only exits in T24 \\
\hline 85 & P78417 & GSTO1 & NO & no significant & YES & only exits in T24 \\
\hline 86 & P68871 & HBB & NO & no significant & YES & only exits in T24 \\
\hline 87 & P12277 & KCRB & NO & no significant & YES & only exits in T24 \\
\hline 88 & P06732 & KCRM & NO & no significant & YES & only exits in T24 \\
\hline 89 & Q96G25 & MED8 & NO & no significant & YES & only exits in T24 \\
\hline 90 & P05121 & PAI1 & NO & no significant & YES & only exits in T24 \\
\hline 91 & Q9UNA4 & POLI & NO & no significant & YES & only exits in T24 \\
\hline 92 & P27169 & PON1 & $\mathrm{NO}$ & no significant & YES & only exits in T24 \\
\hline 93 & P60900 & PSA6 & NO & no significant & YES & only exits in T24 \\
\hline 94 & O00560 & SDCB1 & NO & no significant & YES & only exits in T24 \\
\hline 95 & Q9P273 & TEN3 & NO & no significant & YES & only exits in T24 \\
\hline 96 & P01033 & TIMP1 & NO & no significant & YES & only exits in T24 \\
\hline 97 & P16035 & TIMP2 & NO & no significant & YES & only exits in T24 \\
\hline 98 & O00755 & WNT7A & NO & no significant & YES & only exits in T24 \\
\hline 99 & Р04040 & CATA & NO & no significant & YES & only exits in SV \\
\hline 100 & P07237 & PDIA1 & NO & no significant & YES & only exits in SV \\
\hline 101 & P25787 & PSA2 & NO & no significant & YES & only exits in SV \\
\hline 102 & P37802 & TAGL2 & NO & no significant & YES & only exits in SV \\
\hline 103 & P13693 & TCTP & $\mathrm{NO}$ & no significant & YES & only exits in SV \\
\hline
\end{tabular}


Table S5. Information of all identified proteins through iTRAQ.

\begin{tabular}{|c|c|c|c|c|c|c|c|c|c|}
\hline NO. & $\begin{array}{r}\text { Swissprot } \\
\text { number }\end{array}$ & $\begin{array}{r}\text { Protein } \\
\text { name }\end{array}$ & $\begin{array}{r}\mathrm{MW} \\
(\mathrm{kDa})\end{array}$ & $\begin{array}{c}\text { Sequence } \\
\text { coverage }\end{array}$ & $\begin{array}{l}\text { classical } \\
\text { secreted } \\
\text { proteins }\end{array}$ & $\begin{array}{r}\text { non- } \\
\text { classical } \\
\text { secreted } \\
\text { proteins }\end{array}$ & $\begin{array}{l}\text { urinary } \\
\text { protein }\end{array}$ & $\begin{array}{r}\text { differe } \\
\text { nt in } \\
5637 \\
\text { vs. SV }\end{array}$ & $\begin{array}{r}\text { different } \\
\text { in } 5637 \\
\text { vs. SV }\end{array}$ \\
\hline 1 & P02751 & FINC & 262.62 & $68.10 \%$ & YES & NO & YES & YES & YES \\
\hline 2 & P07339 & CATD & 44.55 & $62.90 \%$ & YES & NO & YES & $\mathrm{NO}$ & NO \\
\hline 3 & P08670 & VIME & 53.65 & $67.80 \%$ & $\mathrm{NO}$ & YES & NO & $\mathrm{NO}$ & YES \\
\hline 4 & P06733 & ENOA & 47.17 & $69.60 \%$ & NO & YES & YES & $\mathrm{NO}$ & $\mathrm{NO}$ \\
\hline 5 & B1AVU8 & SAP & 58.11 & $64.90 \%$ & NO & NO & NO & $\mathrm{NO}$ & YES \\
\hline 6 & Q15582 & BGH3 & 74.68 & $65.60 \%$ & NO & NO & YES & $\mathrm{NO}$ & YES \\
\hline 7 & $\mathrm{O} 15230$ & LAMA5 & 399.73 & $29.30 \%$ & YES & NO & YES & YES & $\mathrm{NO}$ \\
\hline 8 & G3XAI2 & G3XAI2 & 198.04 & $36.60 \%$ & YES & NO & NO & YES & NO \\
\hline 9 & P00338 & LDHA & 36.69 & $61.10 \%$ & YES & YES & NO & YES & YES \\
\hline 10 & P07900 & HS90A & 84.66 & $54.80 \%$ & YES & NO & YES & NO & NO \\
\hline 11 & P04075 & ALDOA & 39.42 & $77.50 \%$ & YES & NO & YES & YES & NO \\
\hline 12 & P07858 & CATB & 37.82 & $48.40 \%$ & YES & NO & YES & $\mathrm{NO}$ & YES \\
\hline 13 & O75369 & FLNB & 278.16 & $46.00 \%$ & $\mathrm{NO}$ & NO & YES & YES & YES \\
\hline 14 & P00558 & PGK1 & 44.62 & $62.60 \%$ & YES & NO & YES & $\mathrm{NO}$ & YES \\
\hline 15 & P07737 & PROF1 & 15.05 & $70.00 \%$ & NO & NO & YES & $\mathrm{NO}$ & YES \\
\hline 16 & Q92626 & PXDN & 165.28 & $44.40 \%$ & YES & NO & YES & $\mathrm{NO}$ & YES \\
\hline 17 & Q16787 & LAMA3 & 366.64 & $18.60 \%$ & YES & NO & YES & $\mathrm{NO}$ & YES \\
\hline 18 & P53634 & CATC & 51.85 & $44.10 \%$ & NO & NO & YES & YES & NO \\
\hline 19 & B4DR87 & B4DR87 & 83.55 & $43.50 \%$ & NO & NO & $\mathrm{NO}$ & NO & NO \\
\hline 20 & P16035 & TIMP2 & 24.40 & $55.50 \%$ & YES & NO & YES & YES & YES \\
\hline 21 & Q02388 & CO7A1 & 295.22 & $23.50 \%$ & $\mathrm{NO}$ & NO & YES & YES & NO \\
\hline 22 & B4E1N2 & B4E1N2 & 29.13 & $49.10 \%$ & YES & NO & NO & $\mathrm{NO}$ & YES \\
\hline 23 & B4DR52 & B4DR52 & 13.91 & $39.80 \%$ & NO & YES & NO & YES & YES \\
\hline 24 & Q13751 & LAMB3 & 129.57 & $34.30 \%$ & NO & NO & NO & $\mathrm{NO}$ & NO \\
\hline 25 & Р07093 & GDN & 44.00 & $32.70 \%$ & NO & NO & NO & YES & $\mathrm{NO}$ \\
\hline 26 & P06748 & NPM & 32.58 & $32.70 \%$ & YES & YES & $\mathrm{NO}$ & $\mathrm{NO}$ & $\mathrm{NO}$ \\
\hline 27 & P08729 & $\mathrm{K} 2 \mathrm{C} 7$ & 51.39 & $45.60 \%$ & YES & NO & NO & $\mathrm{NO}$ & NO \\
\hline 28 & O60568 & PLOD3 & 84.79 & $43.50 \%$ & YES & NO & YES & $\mathrm{NO}$ & $\mathrm{NO}$ \\
\hline 29 & P25391 & LAMA1 & 337.08 & $18.20 \%$ & NO & NO & NO & YES & $\mathrm{NO}$ \\
\hline 30 & Q01105 & SET & 33.49 & $35.20 \%$ & NO & NO & NO & NO & $\mathrm{NO}$ \\
\hline 31 & E7ET40 & E7ET40 & 46.92 & $46.90 \%$ & YES & NO & NO & YES & YES \\
\hline 32 & P07686 & HEXB & 63.11 & $31.30 \%$ & NO & NO & YES & NO & NO \\
\hline 33 & Р06744 & G6PI & 63.15 & $35.30 \%$ & NO & NO & YES & YES & YES \\
\hline 34 & P00352 & AL1A1 & 54.86 & $56.50 \%$ & $\mathrm{NO}$ & YES & YES & NO & NO \\
\hline 35 & P16070 & CD44 & 81.54 & $8.09 \%$ & YES & NO & YES & NO & YES \\
\hline 36 & P53396 & ACLY & 120.84 & $32.30 \%$ & $\mathrm{NO}$ & YES & YES & NO & NO \\
\hline 37 & Q13753 & LAMC2 & 130.98 & $24.80 \%$ & YES & NO & NO & NO & NO \\
\hline 38 & P28799 & GRN & 63.54 & $36.40 \%$ & NO & NO & YES & $\mathrm{NO}$ & NO \\
\hline 39 & P62258 & $1433 \mathrm{E}$ & 29.18 & $57.60 \%$ & NO & NO & NO & $\mathrm{NO}$ & $\mathrm{NO}$ \\
\hline 40 & P10619 & PPGB & 54.47 & $36.70 \%$ & YES & NO & YES & NO & $\mathrm{NO}$ \\
\hline 41 & P24592 & IBP6 & 25.32 & $34.20 \%$ & YES & NO & YES & NO & YES \\
\hline 42 & P08727 & $\mathrm{K} 1 \mathrm{C} 19$ & 44.11 & $54.50 \%$ & $\mathrm{NO}$ & YES & YES & NO & YES \\
\hline 43 & P19338 & NUCL & 76.62 & $28.30 \%$ & NO & NO & YES & $\mathrm{NO}$ & NO \\
\hline 44 & P07711 & CATL1 & 37.56 & $39.90 \%$ & NO & NO & YES & NO & $\mathrm{NO}$ \\
\hline 45 & Q13421 & MSLN & 68.99 & $37.50 \%$ & YES & NO & YES & NO & YES \\
\hline 46 & P21980 & TGM2 & 77.33 & $35.80 \%$ & $\mathrm{NO}$ & YES & NO & $\mathrm{NO}$ & $\mathrm{NO}$ \\
\hline 47 & P15311 & EZRI & 69.41 & $38.10 \%$ & YES & YES & YES & $\mathrm{NO}$ & YES \\
\hline 48 & P12814 & ACTN1 & 103.06 & $53.10 \%$ & NO & NO & NO & NO & YES \\
\hline 4 & P62805 & $\mathrm{H} 4$ & 11.37 & $59.20 \%$ & YES & NO & YES & $\mathrm{O}$ & YES \\
\hline
\end{tabular}




\begin{tabular}{|c|c|c|c|c|c|c|c|c|c|}
\hline 50 & P01034 & CYTC & 15.80 & $63.00 \%$ & $\mathrm{NO}$ & NO & YES & $\mathrm{NO}$ & YES \\
\hline 51 & P13796 & PLSL & 70.29 & $38.30 \%$ & YES & YES & YES & YES & YES \\
\hline 52 & P31431 & SDC4 & 21.64 & $13.10 \%$ & $\mathrm{NO}$ & NO & YES & $\mathrm{NO}$ & YES \\
\hline 53 & P43490 & NAMPT & 55.52 & $45.80 \%$ & $\mathrm{NO}$ & NO & YES & $\mathrm{NO}$ & $\mathrm{NO}$ \\
\hline 54 & O60506 & HNRPQ & 69.60 & $29.10 \%$ & YES & NO & NO & NO & NO \\
\hline 55 & E9PKP4 & E9PKP4 & 13.25 & $52.20 \%$ & $\mathrm{NO}$ & NO & NO & NO & NO \\
\hline 56 & P12004 & PCNA & 28.77 & $50.20 \%$ & YES & YES & NO & NO & $\mathrm{NO}$ \\
\hline 57 & P15586 & GNS & 62.08 & $23.00 \%$ & $\mathrm{NO}$ & NO & YES & NO & $\mathrm{NO}$ \\
\hline 58 & P08581 & MET & 155.54 & $19.40 \%$ & NO & NO & NO & YES & YES \\
\hline 59 & Q14118 & DAG1 & 97.44 & $27.70 \%$ & $\mathrm{NO}$ & NO & YES & $\mathrm{NO}$ & NO \\
\hline 60 & P25787 & PSA2 & 25.90 & $41.90 \%$ & NO & NO & YES & NO & $\mathrm{NO}$ \\
\hline 61 & Q14112 & NID2 & 151.25 & $19.60 \%$ & $\mathrm{NO}$ & NO & YES & YES & YES \\
\hline 62 & O00469 & PLOD2 & 84.69 & $26.50 \%$ & NO & NO & NO & $\mathrm{NO}$ & $\mathrm{NO}$ \\
\hline 63 & B7Z2S5 & B2R5P6 & 70.91 & $50.50 \%$ & NO & NO & NO & NO & NO \\
\hline 64 & Q8NBP7 & PCSK9 & 74.28 & $25.70 \%$ & NO & NO & NO & NO & $\mathrm{NO}$ \\
\hline 65 & O60701 & UGDH & 55.03 & $47.00 \%$ & $\mathrm{NO}$ & NO & YES & $\mathrm{NO}$ & $\mathrm{NO}$ \\
\hline 66 & O14773 & TPP1 & 61.25 & $33.90 \%$ & YES & NO & YES & NO & $\mathrm{NO}$ \\
\hline 67 & P12956 & XRCC6 & 69.85 & $37.90 \%$ & YES & NO & NO & YES & YES \\
\hline 68 & B4DTG2 & B4DTG2 & 50.12 & $27.90 \%$ & NO & YES & NO & $\mathrm{NO}$ & YES \\
\hline 69 & P13611 & CSPG2 & 372.81 & $5.57 \%$ & NO & NO & YES & $\mathrm{NO}$ & $\mathrm{NO}$ \\
\hline 70 & P39060 & COIA1 & 178.19 & $9.64 \%$ & YES & NO & YES & $\mathrm{NO}$ & $\mathrm{NO}$ \\
\hline 71 & P35052 & GPC1 & 61.68 & $31.00 \%$ & YES & NO & YES & YES & NO \\
\hline 72 & P08758 & ANXA5 & 35.94 & $59.70 \%$ & YES & YES & YES & $\mathrm{NO}$ & $\mathrm{NO}$ \\
\hline 73 & P61769 & B2MG & 13.71 & $54.60 \%$ & $\mathrm{NO}$ & NO & YES & YES & YES \\
\hline 74 & P31946 & 1433B & 28.08 & $45.50 \%$ & NO & NO & YES & $\mathrm{NO}$ & $\mathrm{NO}$ \\
\hline 75 & O60218 & AK1BA & 36.02 & $37.30 \%$ & YES & NO & YES & $\mathrm{NO}$ & $\mathrm{NO}$ \\
\hline 76 & P47895 & AL1A3 & 56.11 & $28.10 \%$ & NO & NO & NO & $\mathrm{NO}$ & YES \\
\hline 77 & P28066 & PSA5 & 26.41 & $50.60 \%$ & YES & NO & YES & $\mathrm{NO}$ & NO \\
\hline 78 & P62979 & RS27A & 17.97 & $41.00 \%$ & YES & YES & NO & NO & $\mathrm{NO}$ \\
\hline 79 & B7Z1I9 & B7Z9I3 & 39.01 & $37.40 \%$ & NO & NO & NO & NO & NO \\
\hline 80 & D6RHI9 & D6RHI9 & 29.48 & $45.30 \%$ & YES & NO & NO & $\mathrm{NO}$ & $\mathrm{NO}$ \\
\hline 81 & P62158 & CALM & 16.84 & $36.90 \%$ & NO & YES & YES & NO & YES \\
\hline 82 & P11413 & G6PD & 59.26 & $36.50 \%$ & NO & NO & YES & $\mathrm{NO}$ & $\mathrm{NO}$ \\
\hline 83 & P23526 & SAHH & 47.72 & $25.00 \%$ & YES & YES & YES & $\mathrm{NO}$ & YES \\
\hline 84 & P00750 & TPA & 62.92 & $34.30 \%$ & NO & NO & NO & YES & YE \\
\hline 85 & P31947 & $1433 \mathrm{~S}$ & 27.77 & $50.00 \%$ & NO & NO & NO & YES & YE \\
\hline 86 & B4DY08 & B4DY08 & 33.67 & $23.60 \%$ & YES & YES & NO & $\mathrm{NO}$ & $\mathrm{NO}$ \\
\hline 87 & Q9Y287 & ITM2B & 30.34 & $27.40 \%$ & YES & YES & YES & NO & NO \\
\hline 88 & Q92688 & AN32B & 28.79 & $27.50 \%$ & NO & NO & NO & $\mathrm{NO}$ & $\mathrm{NO}$ \\
\hline 89 & E9PJY4 & E9PLK3 & 103.28 & $25.70 \%$ & NO & NO & NO & NO & YES \\
\hline 90 & G3XAD8 & G3XAD8 & 62.64 & $22.40 \%$ & YES & NO & NO & $\mathrm{NO}$ & $\mathrm{NO}$ \\
\hline 91 & P08603 & CFAH & 139.10 & $27.50 \%$ & NO & NO & YES & $\mathrm{NO}$ & $\mathrm{NO}$ \\
\hline 92 & Q16610 & ECM1 & 60.67 & $22.40 \%$ & NO & NO & YES & $\mathrm{NO}$ & $\mathrm{NO}$ \\
\hline 93 & Q9HB40 & RISC & 50.83 & $26.10 \%$ & YES & NO & YES & YES & $\mathrm{NO}$ \\
\hline 94 & Q99538 & LGMN & 49.41 & $32.80 \%$ & NO & NO & YES & YES & NO \\
\hline 95 & Q92824 & PCSK5 & 206.93 & $8.49 \%$ & NO & NO & NO & $\mathrm{NO}$ & $\mathrm{NO}$ \\
\hline 96 & Q14764 & MVP & 99.33 & $23.60 \%$ & $\mathrm{NO}$ & NO & YES & $\mathrm{NO}$ & NO \\
\hline 97 & P00736 & $\mathrm{C} 1 \mathrm{R}$ & 80.12 & $32.50 \%$ & NO & NO & NO & YES & YES \\
\hline 98 & G3V1V0 & G8JLA2 & 17.09 & $77.60 \%$ & $\mathrm{NO}$ & NO & NO & $\mathrm{NO}$ & $\mathrm{NO}$ \\
\hline 99 & Q04828 & $\mathrm{AK} 1 \mathrm{C} 1$ & 36.79 & $47.70 \%$ & YES & NO & YES & $\mathrm{NO}$ & $\mathrm{NO}$ \\
\hline 100 & J3QRS3 & J3QRS3 & 19.80 & $55.40 \%$ & $\mathrm{NO}$ & YES & NO & $\mathrm{NO}$ & NO \\
\hline 101 & Q9UNW1 & MINP1 & 55.05 & $19.70 \%$ & NO & NO & YES & $\mathrm{NO}$ & YES \\
\hline 102 & P11717 & MPRI & 274.37 & $10.30 \%$ & YES & NO & YES & YES & NO \\
\hline 103 & P13647 & $\mathrm{K} 2 \mathrm{C} 5$ & 62.38 & $25.40 \%$ & $\mathrm{NO}$ & NO & NO & $\mathrm{NO}$ & YES \\
\hline 104 & G5E928 & G5E928 & 113.74 & $19.60 \%$ & YES & YES & NO & $\mathrm{NO}$ & $\mathrm{NO}$ \\
\hline 105 & Q15904 & VAS1 & 52.03 & $25.50 \%$ & NO & NO & YES & $\mathrm{NO}$ & YE \\
\hline
\end{tabular}




\begin{tabular}{|c|c|c|c|c|c|c|c|c|c|}
\hline 106 & P01892 & $1 \mathrm{~A} 02$ & 40.92 & $25.50 \%$ & YES & NO & $\mathrm{NO}$ & NO & $\mathrm{NO}$ \\
\hline 107 & P62879 & GBB2 & 37.33 & $41.50 \%$ & YES & $\mathrm{NO}$ & YES & NO & $\mathrm{NO}$ \\
\hline 108 & P00450 & CERU & 122.21 & $11.90 \%$ & $\mathrm{NO}$ & $\mathrm{NO}$ & YES & NO & $\mathrm{NO}$ \\
\hline 109 & F5H816 & E9PK47 & 97.15 & $21.90 \%$ & YES & $\mathrm{NO}$ & $\mathrm{NO}$ & NO & $\mathrm{NO}$ \\
\hline 110 & Q8NHP8 & PLBL2 & 65.47 & $20.70 \%$ & $\mathrm{NO}$ & $\mathrm{NO}$ & YES & NO & $\mathrm{NO}$ \\
\hline 111 & F8VV40 & F8VV40 & 69.29 & $23.20 \%$ & YES & YES & $\mathrm{NO}$ & NO & $\mathrm{NO}$ \\
\hline 112 & Q08629 & TICN1 & 49.12 & $25.30 \%$ & YES & $\mathrm{NO}$ & YES & NO & YES \\
\hline 113 & P32119 & PRDX2 & 21.89 & $28.30 \%$ & $\mathrm{NO}$ & YES & YES & NO & $\mathrm{NO}$ \\
\hline 114 & B7ZMG0 & B7ZMG0 & 162.10 & $9.23 \%$ & NO & $\mathrm{NO}$ & $\mathrm{NO}$ & YES & $\mathrm{NO}$ \\
\hline 115 & Q92876 & KLK6 & 26.86 & $26.60 \%$ & $\mathrm{NO}$ & $\mathrm{NO}$ & YES & YES & YES \\
\hline 116 & Q71UI9 & $\mathrm{H} 2 \mathrm{AV}$ & 13.51 & $31.20 \%$ & YES & YES & YES & YES & YES \\
\hline 117 & Q9BTY2 & FUCO2 & 54.07 & $23.80 \%$ & $\mathrm{NO}$ & NO & YES & YES & $\mathrm{NO}$ \\
\hline 118 & Q9BWD1 & THIC & 41.35 & $22.70 \%$ & YES & YES & YES & NO & YES \\
\hline 119 & P10909 & CLUS & 52.50 & $30.50 \%$ & YES & NO & YES & NO & $\mathrm{NO}$ \\
\hline 120 & Q99497 & PARK7 & 19.89 & $64.60 \%$ & $\mathrm{NO}$ & $\mathrm{NO}$ & YES & NO & YES \\
\hline 121 & Q08945 & SSRP1 & 81.08 & $16.90 \%$ & YES & NO & $\mathrm{NO}$ & NO & YES \\
\hline 122 & P15144 & AMPN & 109.54 & $17.10 \%$ & YES & $\mathrm{NO}$ & YES & NO & $\mathrm{NO}$ \\
\hline 123 & P50897 & PPT1 & 34.19 & $35.30 \%$ & YES & NO & YES & NO & NO \\
\hline 124 & Q5W0H4 & Q5W0H4 & 19.60 & $71.80 \%$ & $\mathrm{NO}$ & YES & $\mathrm{NO}$ & NO & $\mathrm{NO}$ \\
\hline 125 & D6RD18 & D6R9P3 & 30.47 & $28.90 \%$ & YES & $\mathrm{NO}$ & $\mathrm{NO}$ & NO & $\mathrm{NO}$ \\
\hline 126 & Q86UX2 & ITIH5 & 104.58 & $18.00 \%$ & $\mathrm{NO}$ & $\mathrm{NO}$ & YES & NO & $\mathrm{NO}$ \\
\hline 127 & B8ZZP4 & B8ZZP4 & 46.38 & $25.00 \%$ & $\mathrm{NO}$ & NO & $\mathrm{NO}$ & NO & NO \\
\hline 128 & P01009 & A1AT & 46.74 & $35.60 \%$ & $\mathrm{NO}$ & $\mathrm{NO}$ & YES & YES & YES \\
\hline 129 & A4D1W8 & A4D1W8 & 25.44 & $15.40 \%$ & NO & NO & $\mathrm{NO}$ & NO & $\mathrm{NO}$ \\
\hline 130 & P06280 & AGAL & 48.77 & $22.80 \%$ & YES & $\mathrm{NO}$ & YES & NO & $\mathrm{NO}$ \\
\hline 131 & Q13510 & ASAH1 & 44.66 & $37.00 \%$ & $\mathrm{NO}$ & $\mathrm{NO}$ & YES & NO & $\mathrm{NO}$ \\
\hline 132 & P54819 & KAD2 & 26.48 & $57.70 \%$ & YES & YES & YES & NO & $\mathrm{NO}$ \\
\hline 133 & P36952 & SPB5 & 42.10 & $29.10 \%$ & $\mathrm{NO}$ & $\mathrm{NO}$ & YES & YES & YES \\
\hline 134 & P55060 & XPO2 & 110.42 & $14.40 \%$ & YES & $\mathrm{NO}$ & YES & NO & $\mathrm{NO}$ \\
\hline 135 & P50502 & F10A1 & 41.33 & $18.20 \%$ & $\mathrm{NO}$ & YES & YES & NO & YES \\
\hline 136 & P46782 & RS5 & 22.88 & $22.50 \%$ & $\mathrm{NO}$ & YES & YES & YES & YES \\
\hline 137 & P24534 & EF1B & 24.76 & $33.80 \%$ & $\mathrm{NO}$ & YES & $\mathrm{NO}$ & YES & NO \\
\hline 138 & P09871 & $\mathrm{C} 1 \mathrm{~S}$ & 76.68 & $16.90 \%$ & $\mathrm{NO}$ & $\mathrm{NO}$ & YES & NO & NO \\
\hline 139 & P34059 & GALNS & 58.03 & $16.50 \%$ & YES & $\mathrm{NO}$ & YES & NO & $\mathrm{NO}$ \\
\hline 140 & P13284 & GILT & 27.96 & $14.80 \%$ & $\mathrm{NO}$ & $\mathrm{NO}$ & YES & NO & YES \\
\hline 141 & P16278 & BGAL & 76.08 & $16.00 \%$ & $\mathrm{NO}$ & $\mathrm{NO}$ & YES & NO & $\mathrm{NO}$ \\
\hline 142 & P54727 & RD23B & 43.17 & $14.40 \%$ & $\mathrm{NO}$ & $\mathrm{NO}$ & YES & NO & $\mathrm{NO}$ \\
\hline 143 & P49788 & TIG1 & 33.29 & $19.00 \%$ & YES & $\mathrm{NO}$ & YES & YES & YES \\
\hline 144 & P02768 & ALBU & 69.37 & $6.57 \%$ & $\mathrm{NO}$ & $\mathrm{NO}$ & YES & NO & $\mathrm{NO}$ \\
\hline 145 & Е9PEP6 & Е9PEP6 & 103.14 & $19.60 \%$ & $\mathrm{NO}$ & $\mathrm{NO}$ & $\mathrm{NO}$ & YES & $\mathrm{NO}$ \\
\hline 146 & F5H098 & F5H098 & 36.43 & $25.30 \%$ & $\mathrm{NO}$ & $\mathrm{NO}$ & $\mathrm{NO}$ & NO & NO \\
\hline 147 & O00462 & MANBA & 100.90 & $14.00 \%$ & $\mathrm{NO}$ & $\mathrm{NO}$ & YES & YES & $\mathrm{NO}$ \\
\hline 148 & O00154 & $\mathrm{BACH}$ & 41.80 & $22.90 \%$ & $\mathrm{NO}$ & YES & $\mathrm{NO}$ & NO & $\mathrm{NO}$ \\
\hline 149 & P41250 & SYG & 83.17 & $16.80 \%$ & $\mathrm{NO}$ & NO & YES & NO & YES \\
\hline 150 & O94907 & DKK1 & 28.67 & $17.70 \%$ & YES & NO & NO & NO & YES \\
\hline 151 & Q12797 & ASPH & 85.86 & $16.20 \%$ & $\mathrm{NO}$ & YES & $\mathrm{NO}$ & NO & $\mathrm{NO}$ \\
\hline 152 & B9ZVX0 & B9ZVX0 & 141.35 & $15.10 \%$ & $\mathrm{NO}$ & NO & $\mathrm{NO}$ & YES & YES \\
\hline 153 & Q9UBX1 & CATF & 53.37 & $14.90 \%$ & YES & $\mathrm{NO}$ & YES & NO & YES \\
\hline 154 & Q05639 & EF1A2 & 50.47 & $52.10 \%$ & YES & $\mathrm{NO}$ & YES & YES & YES \\
\hline 155 & Q9BS26 & ERP44 & 46.97 & $26.80 \%$ & YES & NO & YES & NO & $\mathrm{NO}$ \\
\hline 156 & Q09028 & RBBP4 & 47.66 & $28.00 \%$ & YES & YES & NO & NO & $\mathrm{NO}$ \\
\hline 157 & Q9NZ08 & ERAP1 & 107.24 & $11.50 \%$ & $\mathrm{NO}$ & NO & YES & NO & $\mathrm{NO}$ \\
\hline 158 & B7Z4K6 & B7Z4K6 & 39.58 & $16.10 \%$ & YES & $\mathrm{NO}$ & $\mathrm{NO}$ & NO & $\mathrm{NO}$ \\
\hline 159 & Q9H5V8 & CDCP1 & 92.93 & $10.20 \%$ & YES & NO & $\mathrm{NO}$ & NO & $\mathrm{NO}$ \\
\hline 160 & F8W8J4 & F8W8J4 & 234.72 & $6.79 \%$ & YES & NO & $\mathrm{NO}$ & NO & YES \\
\hline 161 & P62081 & RS7 & 22.13 & $32.00 \%$ & YES & YES & YES & YES & YES \\
\hline
\end{tabular}




\begin{tabular}{|c|c|c|c|c|c|c|c|c|c|}
\hline 162 & H0YMD1 & H0YLU8 & 104.67 & $15.90 \%$ & NO & NO & $\mathrm{NO}$ & $\mathrm{NO}$ & $\mathrm{NO}$ \\
\hline 163 & Q16695 & $\mathrm{H} 31 \mathrm{~T}$ & 15.51 & $21.30 \%$ & $\mathrm{NO}$ & YES & NO & $\mathrm{NO}$ & YES \\
\hline 164 & E9PFH8 & E9PFH8 & 46.84 & $21.30 \%$ & YES & NO & NO & $\mathrm{NO}$ & $\mathrm{NO}$ \\
\hline 165 & O95395 & GCNT3 & 50.86 & $34.00 \%$ & YES & NO & NO & $\mathrm{NO}$ & $\mathrm{NO}$ \\
\hline 166 & P09874 & PARP1 & 113.09 & $14.40 \%$ & $\mathrm{NO}$ & NO & NO & $\mathrm{NO}$ & YES \\
\hline 167 & B4E2S7 & B4E2S7 & 44.96 & $9.92 \%$ & YES & NO & NO & YES & $\mathrm{NO}$ \\
\hline 168 & F5H5D3 & F5H5D3 & 49.90 & $49.70 \%$ & YES & NO & NO & $\mathrm{NO}$ & $\mathrm{NO}$ \\
\hline 169 & P55263 & ADK & 40.55 & $33.10 \%$ & NO & NO & NO & YES & $\mathrm{NO}$ \\
\hline 170 & P16930 & FAAA & 46.38 & $32.90 \%$ & YES & YES & YES & $\mathrm{NO}$ & $\mathrm{NO}$ \\
\hline 171 & Р00441 & SODC & 15.94 & $60.40 \%$ & NO & YES & YES & NO & $\mathrm{NO}$ \\
\hline 172 & Q06210 & GFPT1 & 78.81 & $17.30 \%$ & $\mathrm{NO}$ & NO & NO & $\mathrm{NO}$ & $\mathrm{NO}$ \\
\hline 173 & H0YME6 & H7BYN4 & 109.13 & $11.70 \%$ & NO & YES & NO & NO & YES \\
\hline 174 & Q99519 & NEUR1 & 45.47 & $16.40 \%$ & $\mathrm{NO}$ & NO & YES & $\mathrm{NO}$ & $\mathrm{NO}$ \\
\hline 175 & P48745 & NOV & 39.16 & $14.80 \%$ & YES & NO & YES & NO & $\mathrm{NO}$ \\
\hline 176 & P31942 & HNRH3 & 36.93 & $18.20 \%$ & YES & NO & NO & YES & $\mathrm{NO}$ \\
\hline 177 & B7Z1M1 & B7Z1M1 & 38.89 & $25.70 \%$ & NO & YES & NO & NO & $\mathrm{NO}$ \\
\hline 178 & P04040 & CATA & 59.76 & $16.50 \%$ & NO & NO & YES & $\mathrm{NO}$ & $\mathrm{NO}$ \\
\hline 179 & P25774 & CATS & 37.50 & $25.40 \%$ & YES & NO & YES & NO & $\mathrm{NO}$ \\
\hline 180 & P55010 & IF5 & 49.22 & $15.50 \%$ & YES & NO & NO & $\mathrm{NO}$ & $\mathrm{NO}$ \\
\hline 181 & P16144 & ITB4 & 202.17 & $8.07 \%$ & NO & NO & NO & YES & YES \\
\hline 182 & Q9Y263 & PLAP & 87.16 & $9.31 \%$ & YES & YES & NO & $\mathrm{NO}$ & $\mathrm{NO}$ \\
\hline 183 & F8W1H5 & F8W1H5 & 27.04 & $27.30 \%$ & $\mathrm{NO}$ & YES & NO & NO & $\mathrm{NO}$ \\
\hline 184 & O75629 & CREG1 & 24.08 & $27.30 \%$ & NO & NO & YES & $\mathrm{NO}$ & $\mathrm{NO}$ \\
\hline 185 & B4DDD8 & B3KWE1 & 57.41 & $19.80 \%$ & YES & NO & $\mathrm{NO}$ & YES & YES \\
\hline 186 & B5MBX2 & B5MBX2 & 47.54 & $17.00 \%$ & YES & NO & NO & $\mathrm{NO}$ & $\mathrm{NO}$ \\
\hline 187 & Q9UKZ9 & PCOC2 & 45.72 & $16.90 \%$ & NO & NO & YES & $\mathrm{NO}$ & $\mathrm{NO}$ \\
\hline 188 & P15289 & ARSA & 53.59 & $19.10 \%$ & NO & NO & YES & NO & $\mathrm{NO}$ \\
\hline 189 & P31153 & METK2 & 43.66 & $26.60 \%$ & $\mathrm{NO}$ & YES & YES & $\mathrm{NO}$ & YES \\
\hline 190 & Q13162 & PRDX4 & 30.54 & $31.00 \%$ & NO & NO & NO & $\mathrm{NO}$ & $\mathrm{NO}$ \\
\hline 191 & E7EUT7 & E7EUT7 & 45.42 & $14.20 \%$ & NO & YES & NO & $\mathrm{NO}$ & $\mathrm{NO}$ \\
\hline 192 & P61970 & NTF2 & 14.48 & $74.80 \%$ & YES & YES & YES & YES & $\mathrm{NO}$ \\
\hline 193 & P15559 & NQO1 & 30.87 & $26.60 \%$ & NO & YES & $\mathrm{NO}$ & NO & $\mathrm{NO}$ \\
\hline 194 & P32970 & CD70 & 21.12 & $28.00 \%$ & NO & NO & NO & $\mathrm{NO}$ & $\mathrm{NO}$ \\
\hline 195 & Q14508 & WFDC2 & 12.99 & $33.90 \%$ & NO & NO & YES & YES & YES \\
\hline 196 & P14314 & GLU2B & 59.43 & $15.20 \%$ & NO & NO & YES & YES & $\mathrm{NO}$ \\
\hline 197 & Q04760 & LGUL & 20.78 & $22.30 \%$ & YES & NO & YES & $\mathrm{NO}$ & $\mathrm{NO}$ \\
\hline 198 & A8MUB1 & A8MUB1 & 49.92 & $49.90 \%$ & YES & YES & NO & YES & $\mathrm{NO}$ \\
\hline 199 & B4DJP7 & B4DJP7 & 13.92 & $15.80 \%$ & YES & YES & NO & $\mathrm{NO}$ & YES \\
\hline 200 & P30086 & PEBP1 & 21.06 & $51.30 \%$ & NO & YES & YES & $\mathrm{NO}$ & $\mathrm{NO}$ \\
\hline 201 & B3KSH1 & B3KSH1 & 37.56 & $17.70 \%$ & NO & YES & NO & $\mathrm{NO}$ & YES \\
\hline 202 & B3KV61 & B3KV61 & 47.58 & $31.70 \%$ & NO & NO & NO & $\mathrm{NO}$ & $\mathrm{NO}$ \\
\hline 203 & H3BLZ8 & C9JMU5 & 80.27 & $16.70 \%$ & NO & NO & NO & YES & YES \\
\hline 204 & O60911 & CATL2 & 37.33 & $29.90 \%$ & NO & NO & NO & $\mathrm{NO}$ & YES \\
\hline 205 & P05120 & PAI2 & 46.60 & $26.50 \%$ & NO & YES & YES & NO & $\mathrm{NO}$ \\
\hline 206 & P11279 & LAMP1 & 44.88 & $12.20 \%$ & NO & NO & YES & NO & $\mathrm{NO}$ \\
\hline 207 & P55290 & CAD13 & 78.29 & $6.73 \%$ & NO & NO & YES & $\mathrm{NO}$ & $\mathrm{NO}$ \\
\hline 208 & F5H013 & F5H013 & 8.54 & $27.90 \%$ & YES & YES & NO & NO & YES \\
\hline 209 & E7EWZ6 & E7EWZ6 & 116.04 & $13.00 \%$ & NO & NO & NO & $\mathrm{NO}$ & $\mathrm{NO}$ \\
\hline 210 & P46821 & MAP1B & 270.63 & $5.35 \%$ & YES & NO & NO & NO & YES \\
\hline 211 & P49321 & NASP & 85.24 & $5.84 \%$ & $\mathrm{NO}$ & NO & NO & NO & $\mathrm{NO}$ \\
\hline 212 & E9PFT6 & E9PFT6 & 16.00 & $13.50 \%$ & NO & YES & NO & NO & YES \\
\hline 213 & P28072 & PSB6 & 25.36 & $21.80 \%$ & YES & YES & YES & NO & $\mathrm{NO}$ \\
\hline 214 & Q9BWS9 & CHID1 & 44.94 & $32.10 \%$ & YES & NO & NO & $\mathrm{NO}$ & $\mathrm{NO}$ \\
\hline 215 & Q6P179 & ERAP2 & 110.47 & $10.70 \%$ & $\mathrm{NO}$ & YES & $\mathrm{NO}$ & $\mathrm{NO}$ & $\mathrm{NO}$ \\
\hline 216 & P20591 & MX1 & 75.52 & $14.70 \%$ & NO & NO & NO & $\mathrm{NO}$ & $\mathrm{NO}$ \\
\hline 217 & O00151 & PDLI1 & 36.07 & $32.20 \%$ & $\mathrm{NO}$ & NO & YES & YES & $\mathrm{NO}$ \\
\hline
\end{tabular}




\begin{tabular}{|c|c|c|c|c|c|c|c|c|c|}
\hline 218 & P36871 & PGM1 & 61.45 & $16.40 \%$ & NO & NO & YES & NO & NO \\
\hline 219 & P46060 & RAGP1 & 63.54 & $22.50 \%$ & NO & NO & $\mathrm{NO}$ & $\mathrm{NO}$ & NO \\
\hline 220 & J3КР72 & H7BY58 & 24.64 & $35.30 \%$ & NO & YES & $\mathrm{NO}$ & NO & NO \\
\hline 221 & B7ZBP9 & A6PVN5 & 40.67 & $14.30 \%$ & NO & YES & $\mathrm{NO}$ & YES & YES \\
\hline 222 & P01023 & $\mathrm{A} 2 \mathrm{MG}$ & 163.29 & $1.29 \%$ & NO & NO & YES & NO & NO \\
\hline 223 & P07954 & FUMH & 54.64 & $23.50 \%$ & NO & YES & YES & NO & NO \\
\hline 224 & J3KN08 & J3KN08 & 106.84 & $7.11 \%$ & $\mathrm{NO}$ & NO & $\mathrm{NO}$ & $\mathrm{NO}$ & NO \\
\hline 225 & P05387 & RLA2 & 11.67 & $84.30 \%$ & NO & NO & YES & NO & NO \\
\hline 226 & P02794 & FRIH & 21.23 & $23.50 \%$ & YES & YES & YES & NO & NO \\
\hline 227 & P09429 & HMGB1 & 24.89 & $12.60 \%$ & NO & $\mathrm{NO}$ & YES & NO & YES \\
\hline 228 & Q01469 & FABP5 & 15.16 & $33.30 \%$ & NO & NO & YES & NO & NO \\
\hline 229 & P42330 & AK1C3 & 36.85 & $46.40 \%$ & NO & YES & YES & NO & NO \\
\hline 230 & Q14517 & FAT1 & 506.26 & $2.05 \%$ & NO & NO & $\mathrm{NO}$ & YES & NO \\
\hline 231 & Q32P28 & P3H1 & 83.39 & $8.97 \%$ & NO & NO & NO & NO & YES \\
\hline 232 & P52292 & IMA2 & 57.86 & $13.80 \%$ & YES & YES & $\mathrm{NO}$ & $\mathrm{NO}$ & YES \\
\hline 233 & Q16719 & KYNU & 52.35 & $8.82 \%$ & NO & NO & NO & YES & NO \\
\hline 234 & J3KQ32 & J3KQ32 & 44.75 & $14.70 \%$ & NO & YES & $\mathrm{NO}$ & NO & NO \\
\hline 235 & Q9NZV1 & CRIM1 & 113.73 & $10.20 \%$ & YES & $\mathrm{NO}$ & YES & NO & YES \\
\hline 236 & P29317 & EPHA2 & 108.27 & $7.79 \%$ & YES & NO & YES & NO & YES \\
\hline 237 & Q16543 & CDC37 & 44.47 & $19.60 \%$ & NO & NO & NO & NO & NO \\
\hline 238 & Q9UHL4 & DPP2 & 54.34 & $11.00 \%$ & YES & NO & YES & NO & NO \\
\hline 239 & O94925 & GLSK & 73.46 & $13.60 \%$ & NO & NO & YES & NO & YES \\
\hline 240 & P78539 & SRPX & 51.57 & $14.90 \%$ & NO & NO & YES & YES & NO \\
\hline 241 & Q8WUA8 & TSK & 37.81 & $34.30 \%$ & YES & NO & NO & NO & $\mathrm{NO}$ \\
\hline 242 & J3KR44 & F5GYJ8 & 31.28 & $16.80 \%$ & YES & NO & $\mathrm{NO}$ & NO & YES \\
\hline 243 & F6U211 & F6U211 & 18.90 & $27.90 \%$ & NO & YES & $\mathrm{NO}$ & YES & YES \\
\hline 244 & P04439 & 1A03 & 40.84 & $21.60 \%$ & NO & NO & NO & YES & NO \\
\hline 245 & Q96C86 & DCPS & 38.61 & $21.40 \%$ & YES & YES & $\mathrm{NO}$ & NO & YES \\
\hline 246 & P14174 & MIF & 12.48 & $17.40 \%$ & YES & YES & YES & NO & NO \\
\hline 247 & B7Z403 & B7Z403 & 34.79 & $30.40 \%$ & NO & NO & NO & NO & NO \\
\hline 248 & E9PQ56 & E9PQ56 & 34.53 & $26.90 \%$ & NO & NO & NO & NO & NO \\
\hline 249 & P09529 & INHBB & 45.12 & $10.60 \%$ & YES & NO & NO & YES & $\mathrm{NO}$ \\
\hline 250 & P52597 & HNRPF & 45.67 & $19.50 \%$ & NO & NO & NO & NO & NO \\
\hline 251 & P08133 & ANXA6 & 75.88 & $12.80 \%$ & NO & NO & YES & NO & NO \\
\hline 252 & O75503 & CLN5 & 41.50 & $22.60 \%$ & YES & YES & YES & NO & NO \\
\hline 253 & P04066 & FUCO & 53.69 & $13.50 \%$ & NO & NO & YES & YES & NO \\
\hline 254 & P13928 & ANXA8 & 36.88 & $20.80 \%$ & NO & NO & YES & YES & YES \\
\hline 255 & P46108 & CRK & 33.83 & $14.10 \%$ & NO & NO & YES & NO & NO \\
\hline 256 & Q14914 & PTGR1 & 35.87 & $10.60 \%$ & NO & NO & YES & NO & NO \\
\hline 257 & Q14980 & NUMA1 & 238.26 & $5.67 \%$ & YES & NO & $\mathrm{NO}$ & NO & YES \\
\hline 258 & $\mathrm{O} 43570$ & CAH12 & 39.45 & $17.50 \%$ & NO & NO & YES & NO & NO \\
\hline 259 & O75340 & PDCD6 & 21.87 & $39.30 \%$ & YES & YES & YES & NO & NO \\
\hline 260 & E9PF66 & F5H1G4 & 104.86 & $7.02 \%$ & YES & NO & $\mathrm{NO}$ & YES & NO \\
\hline 261 & P26368 & U2AF2 & 53.50 & $14.30 \%$ & NO & NO & NO & NO & NO \\
\hline 262 & P02760 & AMBP & 39.00 & $16.80 \%$ & YES & NO & YES & NO & NO \\
\hline 263 & F5H877 & F5H877 & 58.40 & $8.67 \%$ & NO & NO & NO & NO & NO \\
\hline 264 & Q9BZZ5 & API5 & 59.01 & $13.20 \%$ & YES & NO & $\mathrm{NO}$ & NO & NO \\
\hline 265 & B0V043 & B0V043 & 140.48 & $5.93 \%$ & NO & $\mathrm{NO}$ & $\mathrm{NO}$ & NO & NO \\
\hline 266 & C9JXX7 & C9JK10 & 126.64 & $13.10 \%$ & YES & YES & $\mathrm{NO}$ & YES & NO \\
\hline 267 & P00966 & ASSY & 46.53 & $17.50 \%$ & NO & NO & YES & YES & YES \\
\hline 268 & E9PCD7 & E9PCD7 & 113.98 & $7.20 \%$ & YES & NO & $\mathrm{NO}$ & NO & NO \\
\hline 269 & P35237 & SPB6 & 42.62 & $14.90 \%$ & NO & NO & NO & NO & YES \\
\hline 270 & E7EUT8 & E7EUT8 & 58.85 & $10.30 \%$ & NO & NO & $\mathrm{NO}$ & NO & YES \\
\hline 271 & P10412 & H14 & 21.87 & $16.90 \%$ & NO & NO & NO & NO & YES \\
\hline 272 & O75367 & $\mathrm{H} 2 \mathrm{AY}$ & 39.62 & $16.90 \%$ & YES & $\mathrm{NO}$ & YES & YES & YES \\
\hline 273 & E7ES43 & E7ES43 & 94.51 & $11.30 \%$ & NO & NO & $\mathrm{NO}$ & NO & NO \\
\hline
\end{tabular}




\begin{tabular}{|c|c|c|c|c|c|c|c|c|c|}
\hline 274 & P30046 & DOPD & 12.71 & $39.00 \%$ & NO & NO & YES & YES & $\mathrm{NO}$ \\
\hline 275 & Q9NSD9 & SYFB & 66.12 & $5.77 \%$ & NO & NO & NO & $\mathrm{NO}$ & YES \\
\hline 276 & P10646 & TFPI1 & 35.02 & $8.88 \%$ & NO & NO & YES & $\mathrm{NO}$ & YES \\
\hline 277 & P10321 & $1 \mathrm{C} 07$ & 40.65 & $21.00 \%$ & YES & $\mathrm{NO}$ & $\mathrm{NO}$ & $\mathrm{NO}$ & $\mathrm{NO}$ \\
\hline 278 & P05386 & RLA1 & 11.51 & $34.20 \%$ & YES & NO & YES & $\mathrm{NO}$ & $\mathrm{NO}$ \\
\hline 279 & Q15102 & PA1B3 & 25.73 & $24.20 \%$ & $\mathrm{NO}$ & NO & YES & $\mathrm{NO}$ & YES \\
\hline 280 & P13798 & ACPH & 81.22 & $5.60 \%$ & YES & YES & YES & $\mathrm{NO}$ & $\mathrm{NO}$ \\
\hline 281 & J3QT22 & J3QT22 & 42.32 & $14.20 \%$ & NO & YES & $\mathrm{NO}$ & NO & YES \\
\hline 282 & Q92597 & NDRG1 & 42.83 & $17.30 \%$ & $\mathrm{NO}$ & YES & YES & $\mathrm{NO}$ & $\mathrm{NO}$ \\
\hline 283 & B7Z2F6 & B7Z2F6 & 102.64 & $4.08 \%$ & $\mathrm{NO}$ & YES & $\mathrm{NO}$ & $\mathrm{NO}$ & $\mathrm{NO}$ \\
\hline 284 & O75531 & BAF & 10.06 & $48.30 \%$ & $\mathrm{NO}$ & YES & YES & $\mathrm{NO}$ & YES \\
\hline 285 & Q8NCC3 & PAG15 & 46.66 & $16.30 \%$ & NO & NO & YES & NO & $\mathrm{NO}$ \\
\hline 286 & E7EQD5 & E7EQD5 & 66.45 & $12.60 \%$ & NO & NO & $\mathrm{NO}$ & $\mathrm{NO}$ & $\mathrm{NO}$ \\
\hline 287 & Q13185 & CBX3 & 20.81 & $26.20 \%$ & NO & YES & $\mathrm{NO}$ & NO & $\mathrm{NO}$ \\
\hline 288 & Q6PCB0 & VWA1 & 46.80 & $11.00 \%$ & NO & $\mathrm{NO}$ & YES & $\mathrm{NO}$ & YES \\
\hline 289 & O95816 & BAG2 & 23.77 & $16.60 \%$ & YES & YES & $\mathrm{NO}$ & NO & $\mathrm{NO}$ \\
\hline 290 & P15924 & DESP & 331.78 & $4.98 \%$ & YES & $\mathrm{NO}$ & YES & $\mathrm{NO}$ & YES \\
\hline 291 & Q53H82 & LACB2 & 32.81 & $33.30 \%$ & $\mathrm{NO}$ & NO & $\mathrm{NO}$ & $\mathrm{NO}$ & $\mathrm{NO}$ \\
\hline 292 & P08243 & ASNS & 64.37 & $8.91 \%$ & YES & NO & YES & $\mathrm{NO}$ & $\mathrm{NO}$ \\
\hline 293 & P09237 & MMP7 & 29.68 & $13.50 \%$ & $\mathrm{NO}$ & $\mathrm{NO}$ & YES & $\mathrm{NO}$ & $\mathrm{NO}$ \\
\hline 294 & P48507 & GSH0 & 30.73 & $21.20 \%$ & NO & YES & $\mathrm{NO}$ & $\mathrm{NO}$ & $\mathrm{NO}$ \\
\hline 295 & Q99988 & GDF15 & 34.14 & $16.60 \%$ & NO & NO & YES & NO & $\mathrm{NO}$ \\
\hline 296 & Q15758 & AAAT & 56.60 & $9.98 \%$ & NO & YES & YES & $\mathrm{NO}$ & $\mathrm{NO}$ \\
\hline 297 & Q8WXF1 & PSPC1 & 58.74 & $14.90 \%$ & NO & NO & NO & $\mathrm{NO}$ & YES \\
\hline 298 & Q63HN8 & RN213 & 591.41 & $1.00 \%$ & YES & NO & $\mathrm{NO}$ & $\mathrm{NO}$ & YES \\
\hline 299 & Q14019 & COTL1 & 15.95 & $38.70 \%$ & $\mathrm{NO}$ & YES & YES & $\mathrm{NO}$ & YES \\
\hline 300 & P33316 & DUT & 26.56 & $21.80 \%$ & YES & YES & NO & NO & $\mathrm{NO}$ \\
\hline 301 & B4DIZ2 & B4DIZ2 & 22.41 & $38.80 \%$ & $\mathrm{NO}$ & YES & $\mathrm{NO}$ & $\mathrm{NO}$ & $\mathrm{NO}$ \\
\hline 302 & E7EPK6 & E7ЕРК6 & 15.42 & $13.10 \%$ & YES & YES & $\mathrm{NO}$ & YES & YES \\
\hline 303 & J3KSN0 & J3KSN0 & 27.04 & $35.40 \%$ & YES & NO & NO & NO & $\mathrm{NO}$ \\
\hline 304 & Q9NRA1 & PDGFC & 39.03 & $9.28 \%$ & NO & NO & YES & YES & $\mathrm{NO}$ \\
\hline 305 & Q92484 & ASM3A & 51.26 & $6.84 \%$ & YES & $\mathrm{NO}$ & YES & NO & $\mathrm{NO}$ \\
\hline 306 & С9J0J7 & С9J0J7 & 20.79 & $33.00 \%$ & NO & YES & NO & $\mathrm{NO}$ & $\mathrm{NO}$ \\
\hline 307 & Q8NCW5 & NNRE & 31.68 & $23.60 \%$ & YES & NO & YES & $\mathrm{NO}$ & $\mathrm{NO}$ \\
\hline 308 & Q9UHD1 & CHRD1 & 37.49 & $22.00 \%$ & NO & YES & NO & $\mathrm{NO}$ & $\mathrm{NO}$ \\
\hline 309 & Q99436 & PSB7 & 29.97 & $31.80 \%$ & NO & NO & YES & $\mathrm{NO}$ & $\mathrm{NO}$ \\
\hline 310 & C9JS14 & B5MCZ9 & 40.88 & $21.10 \%$ & NO & YES & NO & $\mathrm{NO}$ & $\mathrm{NO}$ \\
\hline 311 & Q96RQ9 & OXLA & 62.88 & $9.88 \%$ & YES & NO & $\mathrm{NO}$ & $\mathrm{NO}$ & $\mathrm{NO}$ \\
\hline 312 & P61225 & RAP2B & 20.50 & $31.70 \%$ & NO & YES & YES & YES & $\mathrm{NO}$ \\
\hline 313 & P09525 & ANXA4 & 35.88 & $18.80 \%$ & NO & NO & $\mathrm{NO}$ & $\mathrm{NO}$ & $\mathrm{NO}$ \\
\hline 314 & E1P660 & E1P660 & 50.75 & $7.29 \%$ & $\mathrm{NO}$ & $\mathrm{NO}$ & $\mathrm{NO}$ & $\mathrm{NO}$ & $\mathrm{NO}$ \\
\hline 315 & Q00688 & FKBP3 & 25.18 & $12.50 \%$ & YES & YES & $\mathrm{NO}$ & $\mathrm{NO}$ & YES \\
\hline 316 & P55145 & MANF & 20.70 & $15.90 \%$ & YES & $\mathrm{NO}$ & YES & YES & YES \\
\hline 317 & P05187 & PPB1 & 57.95 & $19.80 \%$ & $\mathrm{NO}$ & NO & YES & YES & YES \\
\hline 318 & F5H6E2 & F5H6E2 & 121.68 & $7.31 \%$ & NO & $\mathrm{NO}$ & $\mathrm{NO}$ & NO & YES \\
\hline 319 & O14929 & HAT1 & 49.51 & $11.90 \%$ & NO & NO & NO & NO & YES \\
\hline 320 & Q96DG6 & CMBL & 28.05 & $19.20 \%$ & NO & NO & YES & NO & $\mathrm{NO}$ \\
\hline 321 & Q9Y5X9 & LIPE & 56.80 & $6.60 \%$ & NO & NO & YES & $\mathrm{NO}$ & YES \\
\hline 322 & O14657 & TOR1B & 37.98 & $11.00 \%$ & YES & $\mathrm{NO}$ & $\mathrm{NO}$ & NO & $\mathrm{NO}$ \\
\hline 323 & P45877 & PPIC & 22.76 & $17.50 \%$ & $\mathrm{NO}$ & NO & YES & NO & $\mathrm{NO}$ \\
\hline 324 & O43240 & KLK10 & 30.17 & $14.10 \%$ & YES & $\mathrm{NO}$ & YES & YES & YES \\
\hline 325 & O75223 & GGCT & 21.01 & $14.90 \%$ & YES & YES & YES & NO & $\mathrm{NO}$ \\
\hline 326 & P11166 & GTR1 & 54.09 & $3.66 \%$ & $\mathrm{NO}$ & $\mathrm{NO}$ & YES & $\mathrm{NO}$ & $\mathrm{NO}$ \\
\hline 327 & O43854 & EDIL3 & 53.77 & $15.20 \%$ & NO & NO & $\mathrm{NO}$ & $\mathrm{NO}$ & YES \\
\hline 328 & P35244 & RFA3 & 13.57 & $47.90 \%$ & NO & $\mathrm{NO}$ & $\mathrm{NO}$ & $\mathrm{NO}$ & $\mathrm{NO}$ \\
\hline 329 & P12830 & CADH1 & 97.46 & $4.65 \%$ & $\mathrm{NO}$ & NO & YES & YES & YES \\
\hline
\end{tabular}




\begin{tabular}{|c|c|c|c|c|c|c|c|c|c|}
\hline 330 & P46063 & RECQ1 & 73.46 & $5.24 \%$ & YES & NO & NO & YES & $\mathrm{NO}$ \\
\hline 331 & Q92882 & OSTF1 & 23.79 & $21.50 \%$ & NO & YES & YES & $\mathrm{NO}$ & $\mathrm{NO}$ \\
\hline 332 & I3L4C9 & F5H6A3 & 56.70 & $10.00 \%$ & NO & NO & NO & $\mathrm{NO}$ & YES \\
\hline 333 & B7Z9D1 & B7Z9D1 & 33.26 & $13.80 \%$ & YES & YES & $\mathrm{NO}$ & $\mathrm{NO}$ & $\mathrm{NO}$ \\
\hline 334 & P13861 & KAP2 & 45.52 & $12.10 \%$ & YES & YES & NO & $\mathrm{NO}$ & YES \\
\hline 335 & P39019 & RS19 & 16.06 & $20.00 \%$ & YES & YES & YES & YES & $\mathrm{NO}$ \\
\hline 336 & Q969P0 & IGSF8 & 65.03 & $7.34 \%$ & $\mathrm{NO}$ & NO & YES & $\mathrm{NO}$ & $\mathrm{NO}$ \\
\hline 337 & Q14192 & FHL2 & 32.19 & $14.30 \%$ & $\mathrm{NO}$ & YES & NO & $\mathrm{NO}$ & YES \\
\hline 338 & P12931 & SRC & 59.84 & $7.65 \%$ & NO & NO & NO & $\mathrm{NO}$ & $\mathrm{NO}$ \\
\hline 339 & Q9NRN5 & OLFL3 & 46.01 & $6.16 \%$ & YES & NO & NO & $\mathrm{NO}$ & $\mathrm{NO}$ \\
\hline 340 & H0YK46 & H0YK46 & 15.55 & $16.80 \%$ & NO & NO & NO & YES & $\mathrm{NO}$ \\
\hline 341 & P35613 & BASI & 33.07 & $5.97 \%$ & NO & NO & YES & $\mathrm{NO}$ & $\mathrm{NO}$ \\
\hline 342 & Q9ULA0 & DNPEP & 52.43 & $26.90 \%$ & NO & NO & $\mathrm{NO}$ & $\mathrm{NO}$ & YES \\
\hline 343 & P22223 & CADH3 & 91.42 & $9.77 \%$ & NO & NO & YES & $\mathrm{NO}$ & YES \\
\hline 344 & C9JP16 & C9JP16 & 46.56 & $13.10 \%$ & $\mathrm{NO}$ & NO & $\mathrm{NO}$ & $\mathrm{NO}$ & $\mathrm{NO}$ \\
\hline 345 & A6NI00 & A6NI00 & 111.03 & $15.90 \%$ & YES & YES & NO & $\mathrm{NO}$ & YES \\
\hline 346 & Q07954 & LRP1 & 504.59 & $1.30 \%$ & YES & NO & YES & $\mathrm{NO}$ & YES \\
\hline 347 & E7ER89 & E7ER89 & 69.50 & $8.41 \%$ & NO & NO & $\mathrm{NO}$ & $\mathrm{NO}$ & NO \\
\hline 348 & E9PNS7 & E9PNS7 & 94.77 & $9.64 \%$ & NO & YES & NO & $\mathrm{NO}$ & YES \\
\hline 349 & Q99999 & G3ST1 & 48.77 & $12.50 \%$ & NO & NO & NO & $\mathrm{NO}$ & $\mathrm{NO}$ \\
\hline 350 & P20933 & ASPG & 37.21 & $18.50 \%$ & YES & NO & YES & $\mathrm{NO}$ & $\mathrm{NO}$ \\
\hline 351 & Q99714 & HCD2 & 26.92 & $19.90 \%$ & NO & NO & YES & $\mathrm{NO}$ & YES \\
\hline 352 & P10451 & OSTP & 35.42 & $13.40 \%$ & NO & NO & YES & $\mathrm{NO}$ & $\mathrm{NO}$ \\
\hline 353 & P28340 & DPOD1 & 123.63 & $2.44 \%$ & NO & NO & NO & $\mathrm{NO}$ & YES \\
\hline 354 & Q8WXX5 & DNJC9 & 29.91 & $26.20 \%$ & $\mathrm{NO}$ & NO & $\mathrm{NO}$ & $\mathrm{NO}$ & $\mathrm{NO}$ \\
\hline 355 & P21283 & VATC1 & 43.94 & $16.80 \%$ & NO & NO & YES & $\mathrm{NO}$ & $\mathrm{NO}$ \\
\hline 356 & G5E9Y2 & G5E9Y2 & 69.92 & $7.21 \%$ & NO & YES & NO & YES & YES \\
\hline 357 & J3KNX3 & J3KNX3 & 85.18 & $6.93 \%$ & YES & NO & NO & $\mathrm{NO}$ & $\mathrm{NO}$ \\
\hline 358 & Q9Y5S9 & RBM8A & 19.89 & $24.70 \%$ & YES & NO & NO & $\mathrm{NO}$ & $\mathrm{NO}$ \\
\hline 359 & G5E960 & G5E960 & 138.80 & $2.74 \%$ & NO & NO & NO & $\mathrm{NO}$ & $\mathrm{NO}$ \\
\hline 360 & O14561 & ACPM & 17.42 & $14.70 \%$ & NO & YES & NO & $\mathrm{NO}$ & $\mathrm{NO}$ \\
\hline 361 & E9PHD4 & A6NDW3 & 71.44 & $3.79 \%$ & NO & NO & NO & YES & YES \\
\hline 362 & B4DTU4 & B4DTU4 & 101.74 & $2.93 \%$ & YES & NO & NO & YES & YES \\
\hline 363 & Q9NR45 & SIAS & 40.31 & $7.80 \%$ & YES & YES & NO & YES & $\mathrm{NO}$ \\
\hline 364 & P39687 & AN32A & 28.59 & $24.50 \%$ & YES & NO & YES & $\mathrm{NO}$ & $\mathrm{NO}$ \\
\hline 365 & Q9Y337 & KLK5 & 32.02 & $16.70 \%$ & YES & NO & NO & YES & YES \\
\hline 366 & Q9NP79 & VTA1 & 33.88 & $15.00 \%$ & NO & NO & YES & $\mathrm{NO}$ & YES \\
\hline 367 & J3KN01 & A8MQ02 & 206.81 & $2.41 \%$ & YES & NO & NO & $\mathrm{NO}$ & YES \\
\hline 368 & P04637 & P53 & 43.65 & $10.90 \%$ & NO & YES & NO & $\mathrm{NO}$ & $\mathrm{NO}$ \\
\hline 369 & Q5T123 & Q5T123 & 10.44 & $33.00 \%$ & $\mathrm{NO}$ & NO & NO & $\mathrm{NO}$ & $\mathrm{NO}$ \\
\hline 370 & B7Z588 & B7Z588 & 47.88 & $10.00 \%$ & NO & YES & NO & $\mathrm{NO}$ & $\mathrm{NO}$ \\
\hline 371 & P62304 & RUXE & 10.80 & $25.00 \%$ & NO & YES & YES & NO & YES \\
\hline 372 & Q68BL7 & OLM2A & 73.06 & $2.76 \%$ & NO & NO & NO & $\mathrm{NO}$ & YES \\
\hline 373 & Q13501 & SQSTM & 47.69 & $4.77 \%$ & YES & YES & NO & NO & $\mathrm{NO}$ \\
\hline 374 & F5GZL7 & F5GZL7 & 838.31 & $0.81 \%$ & YES & NO & NO & $\mathrm{NO}$ & $\mathrm{NO}$ \\
\hline 375 & H3BQZ7 & H3BQZ7 & 85.11 & $6.57 \%$ & NO & NO & NO & $\mathrm{NO}$ & $\mathrm{NO}$ \\
\hline 376 & Q9UHG2 & PCSK1 & 27.37 & $11.90 \%$ & NO & NO & YES & $\mathrm{NO}$ & $\mathrm{NO}$ \\
\hline 377 & P29992 & GNA11 & 42.13 & $6.13 \%$ & YES & NO & YES & NO & $\mathrm{NO}$ \\
\hline 378 & Q9NZN4 & EHD2 & 61.16 & $7.73 \%$ & $\mathrm{NO}$ & NO & $\mathrm{NO}$ & $\mathrm{NO}$ & NO \\
\hline 379 & A2BHY 4 & A2BHY4 & 192.77 & $1.20 \%$ & NO & NO & YES & YES & YES \\
\hline 380 & B3КТC7 & B3КТC7 & 80.60 & $9.14 \%$ & YES & NO & NO & $\mathrm{NO}$ & YES \\
\hline 381 & P39748 & FEN1 & 42.59 & $19.70 \%$ & NO & NO & NO & NO & $\mathrm{NO}$ \\
\hline 382 & Q9NTZ6 & RBM12 & 97.40 & $4.40 \%$ & YES & NO & NO & $\mathrm{NO}$ & $\mathrm{NO}$ \\
\hline 383 & Q13591 & SEM5A & 120.61 & $2.61 \%$ & YES & NO & YES & $\mathrm{NO}$ & $\mathrm{NO}$ \\
\hline 384 & O75351 & VPS4B & 49.30 & $8.11 \%$ & NO & YES & YES & YES & $\mathrm{NO}$ \\
\hline 385 & F5GWE5 & F5GWE5 & 31.81 & $11.90 \%$ & NO & NO & NO & YES & YES \\
\hline
\end{tabular}




\begin{tabular}{|c|c|c|c|c|c|c|c|c|c|}
\hline 386 & A6NDG6 & PGP & 34.01 & $9.66 \%$ & NO & NO & YES & YES & NO \\
\hline 387 & P62851 & RS25 & 13.74 & $14.40 \%$ & NO & NO & YES & NO & NO \\
\hline 388 & Q9BRA2 & TXD17 & 13.94 & $15.40 \%$ & NO & $\mathrm{NO}$ & YES & YES & NO \\
\hline 389 & Q969H8 & $\mathrm{CS} 010$ & 18.79 & $8.67 \%$ & $\mathrm{NO}$ & NO & YES & NO & YES \\
\hline 390 & Q5T760 & Q5T760 & 53.54 & $10.00 \%$ & YES & NO & NO & NO & NO \\
\hline 391 & O43813 & LANC1 & 45.28 & $7.02 \%$ & NO & YES & YES & NO & NO \\
\hline 392 & B7ZC38 & B7ZC38 & 43.98 & $6.25 \%$ & NO & NO & NO & YES & NO \\
\hline 393 & Q6EMK4 & VASN & 71.71 & $4.61 \%$ & NO & NO & YES & YES & YES \\
\hline 394 & P21291 & CSRP1 & 20.57 & $12.40 \%$ & NO & NO & NO & YES & NO \\
\hline 395 & P18754 & $\mathrm{RCC1}$ & 44.97 & $9.26 \%$ & NO & YES & NO & NO & YES \\
\hline 396 & Q9UHY7 & ENOPH & 28.93 & $20.70 \%$ & NO & YES & YES & NO & NO \\
\hline 397 & F5H3L5 & F5H3L5 & 114.74 & $2.60 \%$ & NO & NO & NO & NO & NO \\
\hline 398 & A6NGH2 & A6NGH2 & 35.06 & $12.90 \%$ & NO & YES & NO & NO & NO \\
\hline 399 & O15514 & RPB4 & 16.31 & $25.40 \%$ & NO & YES & NO & NO & NO \\
\hline 400 & O00425 & IF2B3 & 63.71 & $5.18 \%$ & NO & NO & NO & YES & YES \\
\hline 401 & Q8NC51 & PAIRB & 44.97 & $5.39 \%$ & NO & NO & NO & NO & NO \\
\hline 402 & C9JGI3 & C9JGI3 & 49.96 & $7.64 \%$ & NO & YES & NO & NO & YES \\
\hline 403 & P32320 & CDD & 16.18 & $28.10 \%$ & NO & YES & YES & YES & YES \\
\hline 404 & Q96CS3 & FAF2 & 52.62 & $7.87 \%$ & NO & YES & NO & YES & YES \\
\hline 405 & H3BSS5 & H3BSS5 & 26.09 & $10.20 \%$ & NO & YES & NO & NO & NO \\
\hline 406 & O00186 & STXB3 & 67.77 & $3.21 \%$ & NO & NO & YES & NO & NO \\
\hline 407 & E7EWT1 & E7EWT1 & 50.80 & $4.53 \%$ & NO & NO & NO & NO & YES \\
\hline 408 & Q9BXR0 & TGT & 44.05 & $5.21 \%$ & NO & YES & NO & NO & YES \\
\hline 409 & Q93099 & HGD & 49.96 & $5.17 \%$ & NO & YES & YES & NO & NO \\
\hline 410 & Q5ZPR3 & CD276 & 57.23 & $4.49 \%$ & YES & NO & YES & NO & NO \\
\hline 411 & P50895 & BCAM & 67.40 & $7.17 \%$ & NO & NO & YES & NO & YES \\
\hline 412 & B4E2C0 & B4E2C0 & 62.41 & $8.23 \%$ & NO & NO & NO & YES & NO \\
\hline 413 & P38117 & ETFB & 27.84 & $5.49 \%$ & NO & NO & NO & NO & YES \\
\hline 414 & Q9UBI6 & GBG12 & 8.01 & $31.90 \%$ & NO & YES & YES & NO & NO \\
\hline 415 & P16401 & H15 & 22.58 & $11.90 \%$ & NO & NO & YES & YES & YES \\
\hline 416 & Q4G148 & GXLT1 & 50.57 & $4.32 \%$ & NO & NO & NO & YES & NO \\
\hline 417 & Q9UBQ5 & EIF3K & 25.06 & $12.80 \%$ & NO & YES & NO & NO & NO \\
\hline 418 & Q15828 & CYTM & 16.51 & $15.40 \%$ & NO & NO & YES & YES & YES \\
\hline 419 & E7EP74 & E7EP74 & 376.02 & $0.89 \%$ & NO & $\mathrm{NO}$ & NO & NO & YES \\
\hline 420 & P49862 & KLK7 & 27.52 & $13.40 \%$ & NO & NO & NO & YES & YES \\
\hline 421 & P78504 & JAG1 & 133.80 & $2.96 \%$ & NO & $\mathrm{NO}$ & YES & NO & NO \\
\hline 422 & Q14008 & CKAP5 & 225.50 & $1.18 \%$ & YES & NO & NO & NO & YES \\
\hline 423 & Q5JW30 & Q5JW30 & 63.18 & $4.86 \%$ & YES & NO & NO & NO & NO \\
\hline 424 & Q8IWX8 & CHERP & 103.70 & $1.64 \%$ & NO & NO & NO & NO & YES \\
\hline 425 & B8ZZM6 & B8ZZM6 & 30.22 & $15.60 \%$ & YES & $\mathrm{NO}$ & NO & NO & NO \\
\hline 426 & H9KV70 & H9KV70 & 22.59 & $66.50 \%$ & NO & NO & NO & YES & NO \\
\hline 427 & Q99715 & COCA1 & 333.15 & $40.50 \%$ & YES & $\mathrm{NO}$ & YES & YES & NO \\
\hline 428 & P01024 & $\mathrm{CO} 3$ & 187.15 & $58.10 \%$ & NO & NO & YES & YES & NO \\
\hline 429 & P09486 & SPRC & 34.63 & $61.40 \%$ & YES & NO & YES & YES & NO \\
\hline 430 & P05067 & A4 & 86.94 & $33.80 \%$ & NO & NO & YES & YES & YES \\
\hline 431 & P05121 & PAI1 & 45.06 & $56.70 \%$ & $\mathrm{NO}$ & NO & YES & YES & YES \\
\hline 432 & F8W6I7 & F8VRQ1 & 38.75 & $39.70 \%$ & $\mathrm{NO}$ & NO & NO & YES & NO \\
\hline 433 & P24821 & TENA & 240.85 & $31.20 \%$ & YES & NO & YES & YES & NO \\
\hline 434 & P67936 & TPM4 & 28.52 & $35.50 \%$ & NO & NO & NO & YES & NO \\
\hline 435 & P08253 & MMP2 & 73.89 & $45.60 \%$ & NO & NO & YES & YES & NO \\
\hline 436 & P04264 & $\mathrm{K} 2 \mathrm{C} 1$ & 66.04 & $47.40 \%$ & $\mathrm{NO}$ & NO & YES & YES & NO \\
\hline 437 & P04083 & ANXA1 & 38.72 & $45.70 \%$ & NO & YES & YES & YES & NO \\
\hline 438 & P15121 & ALDR & 35.85 & $55.10 \%$ & YES & NO & YES & YES & YES \\
\hline 439 & Q92820 & GGH & 35.97 & $36.20 \%$ & $\mathrm{NO}$ & NO & YES & YES & NO \\
\hline 440 & P14543 & NID1 & 136.38 & $25.30 \%$ & $\mathrm{NO}$ & NO & YES & YES & YES \\
\hline 441 & P03956 & MMP1 & 54.01 & $48.60 \%$ & $\mathrm{NO}$ & NO & YES & YES & NO \\
\hline
\end{tabular}




\begin{tabular}{|c|c|c|c|c|c|c|c|c|c|}
\hline 442 & Q9GZM7 & TINAL & 52.39 & $45.00 \%$ & $\mathrm{NO}$ & NO & YES & YES & YES \\
\hline 443 & P08572 & $\mathrm{CO} 4 \mathrm{~A} 2$ & 167.56 & $18.00 \%$ & YES & NO & YES & YES & $\mathrm{NO}$ \\
\hline 444 & P23142 & FBLN1 & 77.21 & $42.40 \%$ & $\mathrm{NO}$ & NO & YES & YES & NO \\
\hline 445 & P27797 & CALR & 48.14 & $34.10 \%$ & YES & NO & YES & YES & NO \\
\hline 446 & P06576 & ATPB & 56.56 & $47.60 \%$ & $\mathrm{NO}$ & YES & YES & YES & YES \\
\hline 447 & P09238 & MMP10 & 54.15 & $22.30 \%$ & $\mathrm{NO}$ & NO & YES & YES & NO \\
\hline 448 & Q16363 & LAMA4 & 202.53 & $14.40 \%$ & $\mathrm{NO}$ & NO & YES & YES & YES \\
\hline 449 & Q16658 & FSCN1 & 54.53 & $43.00 \%$ & YES & NO & $\mathrm{NO}$ & YES & $\mathrm{NO}$ \\
\hline 450 & P13010 & XRCC5 & 82.71 & $22.10 \%$ & $\mathrm{NO}$ & NO & $\mathrm{NO}$ & YES & YES \\
\hline 451 & F5H7N9 & F5H7N9 & 43.12 & $34.00 \%$ & NO & NO & $\mathrm{NO}$ & YES & $\mathrm{NO}$ \\
\hline 452 & P08476 & INHBA & 47.44 & $34.70 \%$ & NO & NO & $\mathrm{NO}$ & YES & $\mathrm{NO}$ \\
\hline 453 & G3V1S6 & G3V1S6 & 64.73 & $44.10 \%$ & $\mathrm{NO}$ & NO & NO & YES & $\mathrm{NO}$ \\
\hline 454 & Q9UI42 & CBPA4 & 47.35 & $35.60 \%$ & $\mathrm{NO}$ & NO & YES & YES & $\mathrm{NO}$ \\
\hline 455 & P04792 & HSPB1 & 22.78 & $52.70 \%$ & $\mathrm{NO}$ & YES & YES & YES & NO \\
\hline 456 & P35527 & K1C9 & 62.07 & $38.50 \%$ & YES & NO & YES & YES & NO \\
\hline 457 & P17936 & IBP3 & 31.67 & $36.40 \%$ & $\mathrm{NO}$ & NO & YES & YES & YES \\
\hline 458 & P09668 & CATH & 37.39 & $31.90 \%$ & $\mathrm{NO}$ & NO & YES & YES & NO \\
\hline 459 & P20908 & CO5A1 & 183.56 & $8.43 \%$ & $\mathrm{NO}$ & NO & YES & YES & YES \\
\hline 460 & B1AHL2 & B1AHL2 & 78.33 & $32.50 \%$ & $\mathrm{NO}$ & NO & NO & YES & NO \\
\hline 461 & Q96KP4 & CNDP2 & 52.88 & $44.60 \%$ & YES & NO & YES & YES & NO \\
\hline 462 & P54136 & SYRC & 75.38 & $16.50 \%$ & YES & NO & YES & YES & YES \\
\hline 463 & Q93063 & EXT2 & 82.26 & $17.80 \%$ & YES & YES & $\mathrm{NO}$ & YES & NO \\
\hline 464 & P61158 & ARP3 & 47.37 & $37.80 \%$ & $\mathrm{NO}$ & NO & YES & YES & YES \\
\hline 465 & Q8IXL6 & DMP4 & 66.24 & $14.40 \%$ & $\mathrm{NO}$ & NO & NO & YES & NO \\
\hline 466 & O95782 & AP2A1 & 107.55 & $13.60 \%$ & YES & NO & $\mathrm{NO}$ & YES & YES \\
\hline 467 & P46777 & RL5 & 34.36 & $28.60 \%$ & $\mathrm{NO}$ & NO & YES & YES & YES \\
\hline 468 & Q96RW7 & HMCN1 & 613.38 & $2.91 \%$ & $\mathrm{NO}$ & NO & YES & YES & NO \\
\hline 469 & P35908 & K22E & 65.43 & $20.80 \%$ & $\mathrm{NO}$ & NO & YES & YES & NO \\
\hline 470 & B3KS31 & B3KS31 & 49.86 & $38.80 \%$ & YES & YES & NO & YES & NO \\
\hline 471 & Q16394 & EXT1 & 86.26 & $13.50 \%$ & $\mathrm{NO}$ & NO & $\mathrm{NO}$ & YES & NO \\
\hline 472 & Q8NES3 & LFNG & 41.77 & $21.60 \%$ & $\mathrm{NO}$ & NO & YES & YES & NO \\
\hline 473 & B0YIW6 & B0YIW6 & 57.21 & $13.90 \%$ & NO & NO & NO & YES & YES \\
\hline 474 & P09341 & GROA & 11.30 & $30.80 \%$ & $\mathrm{NO}$ & NO & YES & YES & YES \\
\hline 475 & P42785 & PCP & 55.80 & $17.10 \%$ & $\mathrm{NO}$ & NO & YES & YES & YES \\
\hline 476 & C9JFR7 & C9JFR7 & 11.75 & $25.70 \%$ & $\mathrm{NO}$ & NO & NO & YES & YES \\
\hline 477 & Q9BZM5 & N2DL2 & 27.37 & $12.60 \%$ & $\mathrm{NO}$ & NO & $\mathrm{NO}$ & YES & NO \\
\hline 478 & P02538 & K2C6A & 60.05 & $23.80 \%$ & YES & NO & NO & YES & NO \\
\hline 479 & P62280 & RS11 & 18.43 & $38.00 \%$ & $\mathrm{NO}$ & YES & $\mathrm{NO}$ & YES & YES \\
\hline 480 & P02462 & CO4A1 & 160.62 & $7.07 \%$ & YES & NO & YES & YES & NO \\
\hline 481 & Q9UBQ0 & VPS29 & 20.51 & $40.70 \%$ & YES & NO & NO & YES & NO \\
\hline 482 & B4DUC8 & B4DUC8 & 31.24 & $32.00 \%$ & $\mathrm{NO}$ & YES & NO & YES & NO \\
\hline 483 & P21796 & VDAC1 & 30.77 & $18.00 \%$ & $\mathrm{NO}$ & NO & YES & YES & YES \\
\hline 484 & O60763 & USO1 & 107.90 & $6.34 \%$ & $\mathrm{NO}$ & NO & YES & YES & YES \\
\hline 485 & O14672 & ADA10 & 84.14 & $11.40 \%$ & YES & NO & YES & YES & NO \\
\hline 486 & Q24JP5 & T132A & 110.11 & $11.20 \%$ & $\mathrm{NO}$ & NO & YES & YES & NO \\
\hline 487 & P62277 & RS13 & 17.22 & $29.10 \%$ & $\mathrm{NO}$ & YES & YES & YES & NO \\
\hline 488 & B4DER1 & B4DER1 & 134.47 & $9.66 \%$ & $\mathrm{NO}$ & NO & NO & YES & YES \\
\hline 489 & Q7Z304 & MAMC2 & 77.56 & $10.60 \%$ & $\mathrm{NO}$ & NO & $\mathrm{NO}$ & YES & $\mathrm{NO}$ \\
\hline 490 & F2Z2Y0 & F5H1Z7 & 85.21 & $13.90 \%$ & YES & NO & $\mathrm{NO}$ & YES & NO \\
\hline 491 & P45452 & MMP13 & 53.82 & $16.60 \%$ & YES & NO & NO & YES & NO \\
\hline 492 & P42830 & CXCL5 & 11.97 & $36.00 \%$ & $\mathrm{NO}$ & NO & $\mathrm{NO}$ & YES & YES \\
\hline 493 & P15151 & PVR & 45.30 & $6.47 \%$ & $\mathrm{NO}$ & NO & YES & YES & YES \\
\hline 494 & O15212 & PFD6 & 14.58 & $15.50 \%$ & $\mathrm{NO}$ & YES & $\mathrm{NO}$ & YES & YES \\
\hline 495 & P16870 & CBPE & 53.15 & $10.10 \%$ & $\mathrm{NO}$ & NO & YES & YES & NO \\
\hline 496 & P50570 & DYN2 & 98.07 & $7.36 \%$ & $\mathrm{NO}$ & NO & YES & YES & YES \\
\hline 497 & P61812 & TGFB2 & 47.75 & $9.42 \%$ & NO & NO & $\mathrm{NO}$ & YES & $\mathrm{NO}$ \\
\hline
\end{tabular}




\begin{tabular}{|c|c|c|c|c|c|c|c|c|c|}
\hline 498 & B0S872 & B0S872 & 34.23 & $14.30 \%$ & NO & NO & $\mathrm{NO}$ & YES & YES \\
\hline 499 & Q9NYQ8 & FAT2 & 479.31 & $2.87 \%$ & NO & NO & NO & YES & $\mathrm{NO}$ \\
\hline 500 & O60687 & SRPX2 & 52.97 & $24.30 \%$ & NO & NO & YES & YES & $\mathrm{NO}$ \\
\hline 501 & P49411 & EFTU & 49.54 & $11.30 \%$ & NO & YES & YES & YES & YES \\
\hline 502 & P55001 & MFAP2 & 20.83 & $20.20 \%$ & NO & NO & NO & YES & NO \\
\hline 503 & Q9UKP4 & ATS7 & 184.09 & $1.42 \%$ & NO & NO & NO & YES & $\mathrm{NO}$ \\
\hline 504 & Q13217 & DNJC3 & 57.58 & $12.10 \%$ & NO & NO & NO & YES & $\mathrm{NO}$ \\
\hline 505 & F5GWS3 & F5GWS3 & 50.99 & $6.76 \%$ & YES & YES & NO & YES & YES \\
\hline 506 & Q9NR99 & MXRA5 & 312.15 & $3.08 \%$ & NO & $\mathrm{NO}$ & YES & YES & $\mathrm{NO}$ \\
\hline 507 & E9PC48 & Е9PC48 & 38.01 & $19.10 \%$ & YES & NO & NO & YES & YES \\
\hline 508 & O43681 & ASNA & 38.79 & $10.90 \%$ & NO & YES & YES & YES & YES \\
\hline 509 & P09758 & TACD2 & 35.71 & $13.00 \%$ & NO & NO & YES & YES & YES \\
\hline 510 & P45880 & VDAC2 & 31.57 & $12.90 \%$ & NO & NO & YES & YES & YES \\
\hline 511 & Q14563 & SEM3A & 88.89 & $5.84 \%$ & YES & NO & YES & YES & $\mathrm{NO}$ \\
\hline 512 & Q9Y2T3 & GUAD & 51.00 & $15.20 \%$ & NO & NO & YES & YES & YES \\
\hline 513 & Q00577 & PURA & 34.91 & $11.80 \%$ & YES & NO & NO & YES & $\mathrm{NO}$ \\
\hline 514 & C9JNT3 & C9JNT3 & 13.68 & $29.70 \%$ & YES & YES & NO & YES & YES \\
\hline 515 & P25685 & DNJB1 & 38.05 & $7.06 \%$ & NO & YES & YES & YES & YES \\
\hline 516 & Q9P2B2 & FPRP & 98.56 & $3.98 \%$ & NO & NO & YES & YES & $\mathrm{NO}$ \\
\hline 517 & P35556 & FBN2 & 314.76 & $2.85 \%$ & NO & NO & NO & YES & $\mathrm{NO}$ \\
\hline 518 & P49748 & ACADV & 70.39 & $8.40 \%$ & NO & NO & YES & YES & YES \\
\hline 519 & B1AHB1 & B1AHB1 & 82.29 & $5.50 \%$ & YES & NO & NO & YES & YES \\
\hline 520 & C9JL12 & C9JL12 & 61.05 & $5.23 \%$ & NO & NO & NO & YES & $\mathrm{NO}$ \\
\hline 521 & P18085 & ARF4 & 20.51 & $40.00 \%$ & NO & YES & YES & YES & $\mathrm{NO}$ \\
\hline 522 & Q99650 & OSMR & 110.51 & $1.94 \%$ & YES & NO & NO & YES & $\mathrm{NO}$ \\
\hline 523 & A2A274 & A2A274 & 85.43 & $6.09 \%$ & NO & NO & NO & YES & $\mathrm{NO}$ \\
\hline 524 & Q86SQ4 & GP126 & 136.70 & $2.62 \%$ & YES & NO & YES & YES & $\mathrm{NO}$ \\
\hline 525 & Q9UBQ6 & EXTL2 & 37.47 & $10.60 \%$ & NO & NO & YES & YES & YES \\
\hline 526 & P09455 & RET1 & 15.85 & $20.70 \%$ & NO & NO & NO & YES & $\mathrm{NO}$ \\
\hline 527 & P61764 & STXB1 & 67.57 & $5.22 \%$ & YES & NO & NO & YES & YES \\
\hline 528 & Q9BZQ8 & NIBAN & 103.13 & $2.16 \%$ & NO & NO & NO & YES & YES \\
\hline 529 & C9IZN5 & H7BYD0 & 13.46 & $32.10 \%$ & YES & NO & NO & YES & NO \\
\hline 530 & B7Z214 & B7Z1C4 & 52.82 & $9.69 \%$ & YES & YES & NO & YES & NO \\
\hline 531 & P07996 & TSP1 & 129.38 & $34.20 \%$ & YES & NO & YES & YES & $\mathrm{NO}$ \\
\hline 532 & Q12805 & FBLN3 & 54.64 & $41.60 \%$ & NO & NO & YES & YES & YES \\
\hline 533 & O43405 & $\mathrm{COCH}$ & 59.48 & $21.60 \%$ & YES & NO & YES & YES & $\mathrm{NO}$ \\
\hline 534 & P62269 & RS18 & 17.72 & $26.30 \%$ & NO & NO & YES & YES & YES \\
\hline 535 & O95336 & 6PGL & 27.55 & $41.50 \%$ & NO & YES & YES & YES & YES \\
\hline 536 & P05198 & IF2A & 36.11 & $16.80 \%$ & NO & NO & NO & YES & YES \\
\hline 537 & O15145 & ARPC3 & 20.55 & $16.90 \%$ & YES & YES & YES & YES & NO \\
\hline 538 & P33908 & MA1A1 & 72.97 & $7.96 \%$ & NO & NO & YES & YES & $\mathrm{NO}$ \\
\hline 539 & P30040 & ERP29 & 28.99 & $22.60 \%$ & $\mathrm{NO}$ & NO & YES & YES & $\mathrm{NO}$ \\
\hline 540 & P49419 & AL7A1 & 58.49 & $13.00 \%$ & YES & YES & YES & YES & YES \\
\hline 541 & C9JD73 & C9J177 & 41.57 & $20.10 \%$ & NO & YES & NO & YES & YES \\
\hline 542 & P05156 & CFAI & 65.75 & $4.46 \%$ & NO & NO & YES & YES & YES \\
\hline 543 & B3KSQ1 & B3KSQ1 & 36.04 & $5.26 \%$ & NO & NO & NO & YES & YES \\
\hline 544 & Q8WVM8 & SCFD1 & 72.38 & $10.70 \%$ & YES & NO & NO & YES & YES \\
\hline 545 & A6NKH4 & A6NKH4 & 59.07 & $8.65 \%$ & YES & NO & NO & YES & YES \\
\hline 546 & Q9UBG0 & MRC2 & 166.67 & $1.96 \%$ & YES & NO & YES & YES & NO \\
\hline 547 & Q01484 & ANK2 & 433.71 & $0.43 \%$ & NO & NO & NO & YES & $\mathrm{NO}$ \\
\hline 548 & O00468 & AGRIN & 214.84 & $43.20 \%$ & YES & NO & YES & $\mathrm{NO}$ & YES \\
\hline 549 & P04406 & G3P & 36.05 & $74.30 \%$ & NO & NO & YES & $\mathrm{NO}$ & YES \\
\hline 550 & P98160 & PGBM & 468.81 & $29.60 \%$ & NO & NO & YES & $\mathrm{NO}$ & YES \\
\hline 551 & P12109 & CO6A1 & 108.53 & $32.60 \%$ & NO & NO & YES & $\mathrm{NO}$ & YES \\
\hline 552 & B4E3Q1 & B4E3Q1 & 109.79 & $40.60 \%$ & YES & NO & NO & $\mathrm{NO}$ & YES \\
\hline 553 & Q5H9B5 & Q5H9A7 & 23.17 & $83.20 \%$ & $\mathrm{NO}$ & NO & NO & $\mathrm{NO}$ & YES \\
\hline
\end{tabular}




\begin{tabular}{|c|c|c|c|c|c|c|c|c|c|}
\hline 554 & P07195 & LDHB & 36.64 & $64.10 \%$ & NO & YES & YES & $\mathrm{NO}$ & YES \\
\hline 555 & P60174 & TPIS & 30.79 & $73.40 \%$ & NO & YES & YES & $\mathrm{NO}$ & YES \\
\hline 556 & Q00610 & CLH1 & 191.62 & $41.90 \%$ & NO & NO & YES & $\mathrm{NO}$ & YES \\
\hline 557 & Q01082 & SPTB2 & 274.61 & $23.30 \%$ & NO & NO & NO & $\mathrm{NO}$ & YES \\
\hline 558 & Q02818 & NUCB1 & 53.88 & $39.70 \%$ & NO & NO & YES & $\mathrm{NO}$ & YES \\
\hline 559 & Q9UBP4 & DKK3 & 38.39 & $45.70 \%$ & YES & NO & YES & $\mathrm{NO}$ & YES \\
\hline 560 & P08107 & HSP71 & 70.05 & $47.90 \%$ & NO & NO & YES & $\mathrm{NO}$ & YES \\
\hline 561 & F8WA83 & B5MCQ5 & 48.12 & $37.70 \%$ & YES & NO & NO & $\mathrm{NO}$ & YES \\
\hline 562 & Q06481 & APLP2 & 86.96 & $23.90 \%$ & NO & NO & YES & $\mathrm{NO}$ & YES \\
\hline 563 & B4DUR8 & B4DUR8 & 60.54 & $39.00 \%$ & NO & NO & NO & $\mathrm{NO}$ & YES \\
\hline 564 & Q14974 & IMB1 & 97.17 & $28.80 \%$ & NO & YES & YES & $\mathrm{NO}$ & YES \\
\hline 565 & G5EA52 & G5EA52 & 56.78 & $41.20 \%$ & NO & NO & NO & $\mathrm{NO}$ & YES \\
\hline 566 & P30530 & UFO & 98.34 & $12.20 \%$ & NO & NO & YES & $\mathrm{NO}$ & YES \\
\hline 567 & B4DTU0 & B4DTU0 & 65.10 & $41.40 \%$ & NO & NO & NO & $\mathrm{NO}$ & YES \\
\hline 568 & P55268 & LAMB2 & 195.98 & $20.50 \%$ & $\mathrm{NO}$ & NO & YES & $\mathrm{NO}$ & YES \\
\hline 569 & P12110 & CO6A2 & 108.58 & $18.40 \%$ & $\mathrm{NO}$ & NO & YES & $\mathrm{NO}$ & YES \\
\hline 570 & Q08211 & DHX9 & 140.96 & $23.30 \%$ & YES & NO & YES & $\mathrm{NO}$ & YES \\
\hline 571 & Q7KZF4 & SND1 & 102.00 & $28.60 \%$ & YES & NO & YES & $\mathrm{NO}$ & YES \\
\hline 572 & Q01813 & К6РP & 85.60 & $20.50 \%$ & $\mathrm{NO}$ & NO & NO & $\mathrm{NO}$ & YES \\
\hline 573 & P19022 & CADH2 & 99.81 & $23.50 \%$ & $\mathrm{NO}$ & NO & YES & $\mathrm{NO}$ & YES \\
\hline 574 & Q9Y240 & CLC11 & 35.69 & $32.80 \%$ & $\mathrm{NO}$ & NO & NO & $\mathrm{NO}$ & YES \\
\hline 575 & P50454 & SERPH & 46.44 & $47.10 \%$ & $\mathrm{NO}$ & NO & YES & $\mathrm{NO}$ & YES \\
\hline 576 & Q86VP6 & CAND1 & 136.38 & $17.10 \%$ & NO & NO & YES & $\mathrm{NO}$ & YES \\
\hline 577 & O75326 & SEM7A & 74.82 & $29.10 \%$ & NO & NO & NO & $\mathrm{NO}$ & YES \\
\hline 578 & O00299 & CLIC1 & 26.92 & $62.20 \%$ & YES & NO & YES & $\mathrm{NO}$ & YES \\
\hline 579 & P78527 & PRKDC & 469.10 & $8.53 \%$ & NO & NO & YES & $\mathrm{NO}$ & YES \\
\hline 580 & P30153 & $2 \mathrm{AAA}$ & 65.31 & $32.80 \%$ & YES & YES & YES & $\mathrm{NO}$ & YE \\
\hline 581 & Q12905 & ILF2 & 43.06 & $42.80 \%$ & NO & NO & NO & $\mathrm{NO}$ & YES \\
\hline 582 & Q16531 & DDB1 & 126.97 & $14.40 \%$ & NO & YES & $\mathrm{NO}$ & NO & YES \\
\hline 583 & H0Y8E6 & H0Y8E6 & 101.90 & $19.10 \%$ & $\mathrm{NO}$ & NO & NO & $\mathrm{NO}$ & YES \\
\hline 584 & B7ZKN0 & B7ZKN0 & 160.02 & $14.20 \%$ & $\mathrm{NO}$ & NO & NO & $\mathrm{NO}$ & YES \\
\hline 585 & Q9Y265 & RUVB1 & 50.23 & $40.10 \%$ & $\mathrm{NO}$ & NO & NO & $\mathrm{NO}$ & YES \\
\hline 586 & P62701 & RS4X & 29.60 & $26.60 \%$ & YES & YES & YES & $\mathrm{NO}$ & YES \\
\hline 587 & D3DRA3 & D3DRA3 & 27.57 & $25.80 \%$ & YES & NO & NO & $\mathrm{NO}$ & YES \\
\hline 588 & P15018 & LIF & 22.01 & $25.20 \%$ & $\mathrm{NO}$ & NO & NO & $\mathrm{NO}$ & YE \\
\hline 589 & P19021 & AMD & 108.33 & $17.60 \%$ & YES & NO & YES & $\mathrm{NO}$ & YES \\
\hline 590 & P49720 & PSB3 & 22.95 & $34.10 \%$ & $\mathrm{NO}$ & NO & YES & $\mathrm{NO}$ & YES \\
\hline 591 & O95407 & TNF6B & 32.68 & $30.30 \%$ & YES & NO & NO & $\mathrm{NO}$ & YES \\
\hline 592 & B4DGU4 & B4DGU4 & 85.50 & $20.30 \%$ & $\mathrm{NO}$ & YES & NO & $\mathrm{NO}$ & YES \\
\hline 593 & Q15019 & SEPT2 & 41.49 & $40.40 \%$ & NO & NO & YES & $\mathrm{NO}$ & YES \\
\hline 594 & G3V3X5 & G3V3X5 & 195.04 & $5.85 \%$ & $\mathrm{NO}$ & NO & NO & $\mathrm{NO}$ & YES \\
\hline 595 & P20700 & LMNB1 & 66.41 & $16.90 \%$ & $\mathrm{NO}$ & NO & NO & $\mathrm{NO}$ & YES \\
\hline 596 & P26639 & SYTC & 83.44 & $16.00 \%$ & $\mathrm{NO}$ & NO & YES & $\mathrm{NO}$ & YES \\
\hline 597 & O15144 & ARPC2 & 34.33 & $34.30 \%$ & NO & NO & YES & $\mathrm{NO}$ & YES \\
\hline 598 & P35555 & FBN1 & 312.23 & $5.54 \%$ & YES & NO & YES & $\mathrm{NO}$ & YES \\
\hline 599 & Q15393 & SF3B3 & 135.58 & $16.70 \%$ & NO & YES & NO & $\mathrm{NO}$ & YES \\
\hline 600 & Q14152 & EIF3A & 166.57 & $8.32 \%$ & NO & NO & NO & $\mathrm{NO}$ & $\mathrm{YE}$ \\
\hline 601 & P62241 & RS8 & 24.21 & $44.70 \%$ & NO & NO & YES & $\mathrm{NO}$ & YE \\
\hline 602 & Q14126 & DSG2 & 122.29 & $16.60 \%$ & NO & NO & YES & $\mathrm{NO}$ & YE \\
\hline 603 & J3KNP4 & J3KNP4 & 92.19 & $19.40 \%$ & NO & NO & NO & $\mathrm{NO}$ & YE \\
\hline 604 & D6RBV2 & D6RBV2 & 40.23 & $34.20 \%$ & YES & NO & NO & $\mathrm{NO}$ & YES \\
\hline 605 & P39023 & RL3 & 46.11 & $21.80 \%$ & YES & YES & NO & $\mathrm{NO}$ & YES \\
\hline 606 & Q92743 & HTRA1 & 51.29 & $24.80 \%$ & NO & NO & YES & $\mathrm{NO}$ & YE \\
\hline 607 & P51149 & RAB7A & 23.49 & $47.30 \%$ & YES & NO & YES & $\mathrm{NO}$ & YE \\
\hline 608 & P52565 & GDIR1 & 23.21 & $18.60 \%$ & NO & NO & YES & NO & YE \\
\hline 609 & P50914 & RL14 & 23.43 & $15.80 \%$ & $\mathrm{NO}$ & NO & YES & $\mathrm{NO}$ & YE \\
\hline
\end{tabular}




\begin{tabular}{|c|c|c|c|c|c|c|c|c|c|}
\hline 610 & P48723 & HSP13 & 51.93 & $17.00 \%$ & YES & NO & $\mathrm{NO}$ & $\mathrm{NO}$ & YES \\
\hline 611 & P10124 & SRGN & 17.65 & $27.80 \%$ & NO & NO & NO & $\mathrm{NO}$ & YES \\
\hline 612 & P06703 & S10A6 & 10.18 & $16.70 \%$ & $\mathrm{NO}$ & YES & YES & $\mathrm{NO}$ & YES \\
\hline 613 & P13497 & BMP1 & 111.25 & $15.40 \%$ & NO & $\mathrm{NO}$ & YES & $\mathrm{NO}$ & YES \\
\hline 614 & P10253 & LYAG & 105.32 & $15.10 \%$ & NO & NO & YES & $\mathrm{NO}$ & YES \\
\hline 615 & O14980 & XPO1 & 123.39 & $20.40 \%$ & NO & YES & YES & $\mathrm{NO}$ & YES \\
\hline 616 & O00764 & PDXK & 35.10 & $23.40 \%$ & NO & YES & YES & $\mathrm{NO}$ & YES \\
\hline 617 & Q8WVQ1 & CANT1 & 44.84 & $28.40 \%$ & NO & YES & YES & $\mathrm{NO}$ & YES \\
\hline 618 & P61604 & $\mathrm{CH} 10$ & 10.93 & $68.60 \%$ & NO & YES & YES & $\mathrm{NO}$ & YES \\
\hline 619 & E9PL22 & E9PL22 & 111.34 & $21.10 \%$ & NO & NO & NO & $\mathrm{NO}$ & YES \\
\hline 620 & Q9Y230 & RUVB2 & 51.16 & $26.10 \%$ & NO & NO & NO & $\mathrm{NO}$ & YES \\
\hline 621 & O00571 & DDX3X & 73.25 & $18.40 \%$ & NO & NO & YES & $\mathrm{NO}$ & YES \\
\hline 622 & O43143 & DHX15 & 90.94 & $17.50 \%$ & NO & NO & NO & $\mathrm{NO}$ & YE \\
\hline 623 & P55884 & EIF3B & 92.48 & $13.40 \%$ & NO & NO & NO & $\mathrm{NO}$ & YES \\
\hline 624 & P36578 & RL4 & 47.70 & $26.90 \%$ & NO & NO & YES & $\mathrm{NO}$ & YES \\
\hline 625 & P23246 & SFPQ & 76.15 & $19.50 \%$ & YES & NO & NO & $\mathrm{NO}$ & YES \\
\hline 626 & Q96QK1 & VPS35 & 91.71 & $18.00 \%$ & YES & NO & YES & $\mathrm{NO}$ & YES \\
\hline 627 & Q15181 & IPYR & 32.66 & $35.30 \%$ & NO & NO & YES & $\mathrm{NO}$ & YES \\
\hline 628 & Q96TA1 & NIBL1 & 84.14 & $16.40 \%$ & NO & NO & YES & $\mathrm{NO}$ & YES \\
\hline 629 & Q9P258 & $\mathrm{RCC} 2$ & 56.08 & $22.20 \%$ & NO & NO & NO & $\mathrm{NO}$ & YE \\
\hline 630 & P47897 & SYQ & 87.80 & $18.10 \%$ & NO & NO & YES & $\mathrm{NO}$ & YE \\
\hline 631 & D6RER5 & D6RER5 & 49.40 & $15.30 \%$ & NO & NO & NO & $\mathrm{NO}$ & YE \\
\hline 632 & Q06323 & PSME1 & 28.72 & $27.30 \%$ & YES & NO & YES & $\mathrm{NO}$ & YES \\
\hline 633 & Q14566 & MCM6 & 92.89 & $14.60 \%$ & NO & NO & NO & $\mathrm{NO}$ & YES \\
\hline 634 & Q99460 & PSMD1 & 105.84 & $19.40 \%$ & NO & NO & YES & $\mathrm{NO}$ & YES \\
\hline 635 & P53621 & COPA & 138.35 & $15.60 \%$ & YES & NO & YES & $\mathrm{NO}$ & YES \\
\hline 636 & Q2VIR3 & IF2GL & 51.23 & $23.10 \%$ & NO & NO & NO & $\mathrm{NO}$ & YES \\
\hline 637 & P12270 & TPR & 267.29 & $4.57 \%$ & NO & NO & NO & $\mathrm{NO}$ & YE \\
\hline 638 & P35221 & CTNA1 & 100.07 & $15.30 \%$ & YES & NO & YES & $\mathrm{NO}$ & YE \\
\hline 639 & Q96P70 & IPO9 & 115.96 & $10.50 \%$ & NO & YES & NO & $\mathrm{NO}$ & YE \\
\hline 640 & P30405 & PPIF & 22.04 & $27.10 \%$ & NO & YES & NO & $\mathrm{NO}$ & YES \\
\hline 641 & P00505 & AATM & 47.52 & $28.60 \%$ & NO & NO & YES & $\mathrm{NO}$ & YES \\
\hline 642 & P07814 & SYEP & 170.59 & $9.79 \%$ & NO & NO & YES & $\mathrm{NO}$ & YES \\
\hline 643 & O43776 & SYNC & 62.94 & $21.00 \%$ & YES & NO & YES & NO & YES \\
\hline 644 & Q9H0U4 & RAB1B & 22.17 & $27.40 \%$ & NO & NO & YES & $\mathrm{NO}$ & YES \\
\hline 645 & C9JZR2 & C9JZR2 & 108.17 & $11.60 \%$ & NO & NO & NO & $\mathrm{NO}$ & YE \\
\hline 646 & P05556 & ITB1 & 88.42 & $13.40 \%$ & NO & NO & YES & $\mathrm{NO}$ & YE \\
\hline 647 & Q14512 & FGFP1 & 26.26 & $20.90 \%$ & NO & NO & NO & $\mathrm{NO}$ & YE \\
\hline 648 & P33993 & MCM7 & 81.31 & $12.70 \%$ & YES & NO & NO & $\mathrm{NO}$ & YES \\
\hline 649 & Q92499 & DDX1 & 82.43 & $19.50 \%$ & NO & NO & YES & $\mathrm{NO}$ & YES \\
\hline 650 & P22234 & PUR6 & 47.08 & $24.90 \%$ & YES & YES & YES & $\mathrm{NO}$ & YES \\
\hline 651 & Q14574 & DSC3 & 99.97 & $5.69 \%$ & NO & NO & YES & $\mathrm{NO}$ & YES \\
\hline 652 & P46778 & RL21 & 18.57 & $20.60 \%$ & NO & YES & NO & $\mathrm{NO}$ & YE \\
\hline 653 & Q92896 & GSLG1 & 134.55 & $9.92 \%$ & NO & NO & YES & $\mathrm{NO}$ & YE \\
\hline 654 & J3KPV4 & J3KPV4 & 96.56 & $20.10 \%$ & NO & NO & NO & $\mathrm{NO}$ & YE \\
\hline 655 & P67775 & PP2AA & 35.59 & $28.20 \%$ & NO & NO & NO & $\mathrm{NO}$ & YE \\
\hline 656 & F8W181 & F8W181 & 32.73 & $15.90 \%$ & NO & YES & NO & $\mathrm{NO}$ & YE \\
\hline 657 & B3KVR1 & B3KVR1 & 24.61 & $18.40 \%$ & NO & NO & NO & $\mathrm{NO}$ & YE \\
\hline 658 & F8VV71 & F8VSD4 & 17.14 & $42.90 \%$ & YES & YES & NO & NO & YES \\
\hline 659 & P61353 & RL27 & 15.80 & $14.00 \%$ & YES & YES & YES & $\mathrm{NO}$ & YE \\
\hline 660 & D3YTI8 & D3YTB1 & 15.86 & $35.30 \%$ & NO & YES & NO & $\mathrm{NO}$ & YE \\
\hline 661 & P22692 & IBP4 & 27.93 & $20.50 \%$ & YES & NO & YES & $\mathrm{NO}$ & YE \\
\hline 662 & Q9UNM6 & PSD13 & 42.95 & $22.90 \%$ & NO & YES & YES & $\mathrm{NO}$ & YES \\
\hline 663 & B5ME19 & B5ME19 & 105.35 & $9.19 \%$ & NO & NO & NO & $\mathrm{NO}$ & YE \\
\hline 664 & Q15046 & SYK & 68.05 & $12.90 \%$ & NO & NO & NO & NO & YE \\
\hline 665 & P10155 & RO60 & 60.67 & $13.80 \%$ & YES & NO & NO & $\mathrm{NO}$ & YE \\
\hline
\end{tabular}




\begin{tabular}{|c|c|c|c|c|c|c|c|c|c|}
\hline 666 & Q6ZRP7 & QSOX2 & 77.53 & $9.17 \%$ & NO & YES & $\mathrm{NO}$ & $\mathrm{NO}$ & YES \\
\hline 667 & O00622 & CYR61 & 42.03 & $17.10 \%$ & NO & NO & YES & $\mathrm{NO}$ & YES \\
\hline 668 & O43747 & AP1G1 & 91.35 & $8.03 \%$ & NO & NO & YES & $\mathrm{NO}$ & YES \\
\hline 669 & O43852 & CALU & 37.11 & $26.70 \%$ & NO & NO & YES & $\mathrm{NO}$ & YES \\
\hline 670 & P60228 & EIF3E & 52.22 & $14.80 \%$ & NO & NO & NO & $\mathrm{NO}$ & YES \\
\hline 671 & Q7Z6Z7 & HUWE1 & 481.89 & $3.57 \%$ & NO & NO & YES & $\mathrm{NO}$ & YES \\
\hline 672 & O00231 & PSD11 & 47.47 & $23.70 \%$ & NO & NO & NO & $\mathrm{NO}$ & YES \\
\hline 673 & Q08257 & QOR & 35.21 & $27.40 \%$ & YES & YES & YES & $\mathrm{NO}$ & YES \\
\hline 674 & Q9BRK5 & CAB45 & 41.81 & $17.40 \%$ & YES & NO & YES & $\mathrm{NO}$ & YES \\
\hline 675 & J3KQU0 & E9PKG6 & 50.20 & $21.60 \%$ & NO & NO & NO & $\mathrm{NO}$ & YES \\
\hline 676 & A6NKB8 & A6NKB8 & 72.60 & $14.60 \%$ & YES & YES & NO & $\mathrm{NO}$ & YES \\
\hline 677 & Q76M96 & CCD80 & 108.18 & $9.47 \%$ & NO & NO & NO & $\mathrm{NO}$ & YES \\
\hline 678 & Q9H4M9 & EHD1 & 60.63 & $17.00 \%$ & NO & NO & YES & $\mathrm{NO}$ & YE \\
\hline 679 & Q68CR9 & Q68CR9 & 57.14 & $20.90 \%$ & YES & NO & NO & $\mathrm{NO}$ & YES \\
\hline 680 & P04843 & RPN1 & 68.57 & $16.50 \%$ & NO & NO & YES & $\mathrm{NO}$ & YES \\
\hline 681 & Q9BPX3 & CND3 & 114.34 & $5.02 \%$ & YES & NO & NO & $\mathrm{NO}$ & YES \\
\hline 682 & O15511 & ARPC5 & 16.32 & $20.50 \%$ & NO & YES & YES & $\mathrm{NO}$ & YES \\
\hline 683 & Q9UQE7 & SMC3 & 141.55 & $7.56 \%$ & YES & NO & NO & $\mathrm{NO}$ & YES \\
\hline 684 & P05997 & $\mathrm{CO} 5 \mathrm{~A} 2$ & 144.91 & $5.14 \%$ & YES & NO & NO & $\mathrm{NO}$ & YES \\
\hline 685 & B4DM74 & B4DM74 & 20.76 & $22.10 \%$ & NO & YES & NO & $\mathrm{NO}$ & YE \\
\hline 686 & Q8IYS5 & OSCAR & 30.48 & $12.80 \%$ & NO & NO & YES & $\mathrm{NO}$ & YE \\
\hline 687 & Q9UN70 & PCDGK & 101.08 & $8.46 \%$ & NO & NO & YES & $\mathrm{NO}$ & YE \\
\hline 688 & Q9UBT2 & SAE2 & 71.23 & $21.30 \%$ & YES & NO & NO & $\mathrm{NO}$ & YES \\
\hline 689 & P27635 & RL10 & 24.60 & $30.40 \%$ & NO & YES & YES & $\mathrm{NO}$ & YES \\
\hline 690 & O95433 & AHSA1 & 38.27 & $15.70 \%$ & NO & NO & NO & $\mathrm{NO}$ & YES \\
\hline 691 & C9IZE4 & C9IZE4 & 45.53 & $8.14 \%$ & NO & NO & NO & $\mathrm{NO}$ & YE \\
\hline 692 & $\mathrm{~A} 2 \mathrm{~A} 2 \mathrm{D} 0$ & $\mathrm{~A} 2 \mathrm{~A} 2 \mathrm{D} 0$ & 17.30 & $25.60 \%$ & NO & YES & NO & $\mathrm{NO}$ & $\mathrm{YE}$ \\
\hline 693 & B2RDM2 & B2RDM2 & 47.63 & $31.20 \%$ & NO & YES & NO & $\mathrm{NO}$ & YE \\
\hline 694 & B4DLZ5 & B4DLZ5 & 37.38 & $12.60 \%$ & NO & YES & NO & $\mathrm{NO}$ & YE \\
\hline 695 & Q9BXJ9 & NAA15 & 101.28 & $6.00 \%$ & NO & NO & NO & $\mathrm{NO}$ & YES \\
\hline 696 & B4DKY1 & B4DKY1 & 85.48 & $11.90 \%$ & NO & NO & NO & $\mathrm{NO}$ & YES \\
\hline 697 & Q7L576 & CYFP1 & 145.19 & $7.90 \%$ & YES & NO & YES & $\mathrm{NO}$ & YES \\
\hline 698 & P50452 & SPB8 & 42.77 & $19.00 \%$ & YES & NO & YES & $\mathrm{NO}$ & YEs \\
\hline 699 & Q99584 & S10AD & 11.47 & $23.50 \%$ & YES & NO & YES & $\mathrm{NO}$ & YE \\
\hline 700 & P41252 & SYIC & 144.50 & $7.37 \%$ & NO & NO & NO & $\mathrm{NO}$ & YE \\
\hline 701 & P47755 & CAZA2 & 32.95 & $39.20 \%$ & NO & NO & YES & $\mathrm{NO}$ & YE \\
\hline 702 & Q6P2Q9 & PRP8 & 273.61 & $5.35 \%$ & NO & NO & NO & $\mathrm{NO}$ & $\mathrm{YL}$ \\
\hline 703 & B4DVY1 & B4DVY1 & 63.97 & $16.40 \%$ & NO & NO & NO & $\mathrm{NO}$ & YE \\
\hline 704 & Q96CW1 & AP2M1 & 49.66 & $9.43 \%$ & YES & NO & $\mathrm{NO}$ & $\mathrm{NO}$ & YES \\
\hline 705 & A6NFX8 & A6NFX8 & 24.33 & $15.50 \%$ & NO & NO & NO & $\mathrm{NO}$ & YES \\
\hline 706 & A8K3Z3 & A8K3Z3 & 45.63 & $20.10 \%$ & NO & YES & NO & $\mathrm{NO}$ & YES \\
\hline 707 & Q99536 & VAT1 & 41.92 & $23.90 \%$ & NO & NO & YES & $\mathrm{NO}$ & YE \\
\hline 708 & B4DNJ6 & B4DNJ6 & 38.44 & $15.20 \%$ & YES & NO & NO & $\mathrm{NO}$ & YE \\
\hline 709 & P14923 & PLAK & 81.75 & $13.00 \%$ & YES & NO & YES & $\mathrm{NO}$ & YE \\
\hline 710 & P62854 & RS26 & 13.02 & $23.50 \%$ & NO & NO & YES & $\mathrm{NO}$ & YE \\
\hline 711 & Q9BQA1 & MEP50 & 36.72 & $8.48 \%$ & NO & YES & NO & $\mathrm{NO}$ & YES \\
\hline 712 & F5H7E2 & F5H7E2 & 117.81 & $8.08 \%$ & YES & NO & NO & $\mathrm{NO}$ & YE \\
\hline 713 & Q92900 & RENT1 & 124.35 & $5.05 \%$ & NO & NO & NO & $\mathrm{NO}$ & YE \\
\hline 714 & O76003 & GLRX3 & 37.43 & $18.50 \%$ & NO & YES & NO & $\mathrm{NO}$ & YE \\
\hline 715 & B7Z815 & B7Z815 & 117.00 & $8.84 \%$ & YES & NO & NO & $\mathrm{NO}$ & YE \\
\hline 716 & Q9UBB4 & ATX10 & 53.49 & $11.60 \%$ & NO & NO & NO & $\mathrm{NO}$ & YE \\
\hline 717 & A6NJA2 & A6NJA2 & 56.07 & $10.50 \%$ & NO & YES & NO & $\mathrm{NO}$ & YE \\
\hline 718 & P17858 & K6PL & 85.02 & $10.30 \%$ & YES & NO & YES & $\mathrm{NO}$ & YE \\
\hline 719 & A0AVT1 & UBA6 & 117.97 & $8.27 \%$ & NO & NO & YES & $\mathrm{NO}$ & YE \\
\hline 720 & P46109 & CRKL & 33.78 & $19.10 \%$ & NO & YES & YES & NO & YE \\
\hline 721 & P37235 & HPCL1 & 22.31 & $21.80 \%$ & NO & YES & YES & $\mathrm{NO}$ & YE \\
\hline
\end{tabular}




\begin{tabular}{|c|c|c|c|c|c|c|c|c|c|}
\hline 722 & B0QYN7 & B0QYN7 & 20.46 & $21.70 \%$ & $\mathrm{NO}$ & $\mathrm{NO}$ & NO & NO & YES \\
\hline 723 & G3V2F7 & G3V2F7 & 16.50 & $4.59 \%$ & $\mathrm{NO}$ & YES & $\mathrm{NO}$ & $\mathrm{NO}$ & YES \\
\hline 724 & F5H897 & F5H897 & 80.11 & $17.20 \%$ & $\mathrm{NO}$ & YES & $\mathrm{NO}$ & $\mathrm{NO}$ & YES \\
\hline 725 & P78324 & SHPS1 & 54.97 & $16.10 \%$ & NO & $\mathrm{NO}$ & YES & NO & YES \\
\hline 726 & Q8NBJ4 & GOLM1 & 45.33 & $13.20 \%$ & $\mathrm{NO}$ & NO & YES & NO & YES \\
\hline 727 & P03950 & ANGI & 16.55 & $33.30 \%$ & NO & NO & YES & NO & YES \\
\hline 728 & Q99471 & PFD5 & 17.33 & $21.40 \%$ & NO & NO & YES & NO & YES \\
\hline 729 & P49767 & VEGFC & 46.88 & $17.40 \%$ & NO & NO & YES & NO & YES \\
\hline 730 & A0AVL1 & A0AVL1 & 90.56 & $5.62 \%$ & NO & NO & $\mathrm{NO}$ & $\mathrm{NO}$ & YES \\
\hline 731 & O00241 & SIRB1 & 43.21 & $12.30 \%$ & NO & NO & YES & NO & YES \\
\hline 732 & O95881 & TXD12 & 19.21 & $19.20 \%$ & NO & NO & NO & NO & YES \\
\hline 733 & B4E3K9 & B3KUK2 & 24.72 & $15.90 \%$ & NO & YES & $\mathrm{NO}$ & NO & YES \\
\hline 734 & P43034 & LIS1 & 46.64 & $10.50 \%$ & NO & NO & $\mathrm{NO}$ & $\mathrm{NO}$ & YES \\
\hline 735 & P63151 & $2 \mathrm{ABA}$ & 51.69 & $11.20 \%$ & NO & NO & YES & NO & YES \\
\hline 736 & C9JNW5 & C9JNW5 & 17.78 & $20.00 \%$ & NO & YES & NO & NO & YES \\
\hline 737 & Q8WUT4 & LRRN4 & 78.84 & $5.27 \%$ & NO & $\mathrm{NO}$ & YES & NO & YES \\
\hline 738 & F5H124 & F5H6P7 & 17.28 & $29.40 \%$ & NO & YES & $\mathrm{NO}$ & $\mathrm{NO}$ & YES \\
\hline 739 & P21583 & SCF & 30.90 & $8.06 \%$ & YES & $\mathrm{NO}$ & $\mathrm{NO}$ & NO & YES \\
\hline 740 & Q9UBF2 & COPG2 & 97.62 & $10.20 \%$ & NO & NO & NO & NO & YES \\
\hline 741 & Q9UGI8 & TES & 48.00 & $15.20 \%$ & NO & NO & NO & NO & YES \\
\hline 742 & P35232 & PHB & 29.80 & $18.80 \%$ & NO & NO & YES & $\mathrm{NO}$ & YES \\
\hline 743 & B2CPU0 & B2CPU0 & 52.82 & $12.60 \%$ & NO & $\mathrm{NO}$ & $\mathrm{NO}$ & NO & YES \\
\hline 744 & Q9H3U1 & UN45A & 103.08 & $7.52 \%$ & NO & YES & NO & NO & YES \\
\hline 745 & B0QY89 & B0QY89 & 66.73 & $8.40 \%$ & NO & YES & $\mathrm{NO}$ & $\mathrm{NO}$ & YES \\
\hline 746 & Q9NPC4 & A4GAT & 40.50 & $15.00 \%$ & YES & YES & $\mathrm{NO}$ & $\mathrm{NO}$ & YES \\
\hline 747 & Q5VYK3 & ECM29 & 204.30 & $2.44 \%$ & YES & $\mathrm{NO}$ & YES & $\mathrm{NO}$ & YES \\
\hline 748 & P08621 & RU17 & 51.56 & $9.61 \%$ & NO & $\mathrm{NO}$ & $\mathrm{NO}$ & NO & YES \\
\hline 749 & P51665 & PSD7 & 37.03 & $16.40 \%$ & YES & NO & YES & $\mathrm{NO}$ & YES \\
\hline 750 & B3KRS5 & B3KRS5 & 55.37 & $11.60 \%$ & NO & NO & $\mathrm{NO}$ & $\mathrm{NO}$ & YES \\
\hline 751 & Q13630 & FCL & 35.89 & $12.80 \%$ & NO & NO & $\mathrm{NO}$ & $\mathrm{NO}$ & YES \\
\hline 752 & B5MC21 & B5MCZ3 & 23.72 & $19.00 \%$ & NO & YES & $\mathrm{NO}$ & NO & YES \\
\hline 753 & Q9UKD2 & MRT4 & 27.56 & $23.80 \%$ & NO & NO & $\mathrm{NO}$ & $\mathrm{NO}$ & YES \\
\hline 754 & P54920 & SNAA & 33.23 & $18.30 \%$ & NO & YES & YES & NO & YES \\
\hline 755 & P21964 & COMT & 30.04 & $19.90 \%$ & NO & NO & YES & $\mathrm{NO}$ & YES \\
\hline 756 & F5GYL8 & F5GYL8 & 60.03 & $5.18 \%$ & NO & NO & $\mathrm{NO}$ & NO & YES \\
\hline 757 & B5ME97 & B5ME97 & 52.59 & $5.15 \%$ & NO & YES & NO & NO & YES \\
\hline 758 & E5RJ48 & E5RGR0 & 24.67 & $25.40 \%$ & NO & YES & $\mathrm{NO}$ & NO & YES \\
\hline 759 & Q13151 & ROA0 & 30.84 & $12.80 \%$ & NO & NO & NO & NO & YES \\
\hline 760 & P04844 & RPN2 & 69.28 & $6.66 \%$ & NO & NO & YES & NO & YES \\
\hline 761 & F8W7S5 & F8W7S5 & 152.47 & $4.13 \%$ & NO & NO & $\mathrm{NO}$ & $\mathrm{NO}$ & YES \\
\hline 762 & Q9P287 & BCCIP & 35.98 & $9.87 \%$ & NO & YES & $\mathrm{NO}$ & NO & YES \\
\hline 763 & F5H4L7 & F5H4L7 & 38.17 & $8.71 \%$ & NO & $\mathrm{NO}$ & NO & NO & YES \\
\hline 764 & J3KNF6 & J3KNF6 & 47.55 & $5.23 \%$ & NO & YES & $\mathrm{NO}$ & $\mathrm{NO}$ & YES \\
\hline 765 & B4DW05 & B4DP75 & 33.30 & $21.80 \%$ & YES & YES & $\mathrm{NO}$ & NO & YES \\
\hline 766 & P36776 & LONM & 106.49 & $3.02 \%$ & NO & NO & YES & $\mathrm{NO}$ & YES \\
\hline 767 & Q2TAY7 & SMU1 & 57.54 & $6.82 \%$ & NO & NO & NO & NO & YES \\
\hline 768 & P46087 & NOP2 & 89.30 & $2.96 \%$ & NO & YES & $\mathrm{NO}$ & $\mathrm{NO}$ & YES \\
\hline 769 & B7Z5W1 & B7Z5W1 & 32.58 & $7.59 \%$ & NO & NO & $\mathrm{NO}$ & NO & YES \\
\hline 770 & Q9GZX9 & TWSG1 & 25.02 & $10.80 \%$ & YES & NO & YES & $\mathrm{NO}$ & YES \\
\hline 771 & B3KVK7 & B3KVK7 & 101.12 & $10.60 \%$ & NO & YES & NO & NO & YES \\
\hline 772 & H7C 579 & H7C579 & 30.61 & $14.70 \%$ & $\mathrm{NO}$ & YES & $\mathrm{NO}$ & NO & YES \\
\hline 773 & Q5JXH7 & Q5JXI8 & 29.16 & $17.50 \%$ & NO & NO & $\mathrm{NO}$ & NO & YES \\
\hline 774 & D6RHU3 & D6RHU3 & 16.72 & $14.70 \%$ & YES & NO & NO & NO & YES \\
\hline 775 & O75153 & CLU & 146.67 & $2.60 \%$ & NO & NO & $\mathrm{NO}$ & NO & YES \\
\hline 776 & B3KS98 & B3KS98 & 39.93 & $7.10 \%$ & NO & NO & NO & NO & YES \\
\hline 777 & P36542 & ATPG & 33.00 & $8.39 \%$ & NO & NO & YES & NO & YES \\
\hline
\end{tabular}




\begin{tabular}{|c|c|c|c|c|c|c|c|c|c|}
\hline 778 & P31040 & DHSA & 72.69 & $3.61 \%$ & YES & NO & YES & NO & YES \\
\hline 779 & Q6P2E9 & EDC4 & 151.66 & $3.21 \%$ & YES & $\mathrm{NO}$ & $\mathrm{NO}$ & $\mathrm{NO}$ & YES \\
\hline 780 & O94826 & TOM70 & 67.46 & $4.11 \%$ & NO & NO & YES & NO & YES \\
\hline 781 & Q99622 & $\mathrm{C} 10$ & 13.18 & $28.60 \%$ & NO & YES & NO & NO & YES \\
\hline 782 & B1AKP7 & B1AKP7 & 44.74 & $7.46 \%$ & NO & YES & $\mathrm{NO}$ & NO & YES \\
\hline 783 & Q6ZR08 & DYH12 & 356.95 & $0.36 \%$ & YES & $\mathrm{NO}$ & $\mathrm{NO}$ & $\mathrm{NO}$ & YES \\
\hline 784 & E9PJD9 & E9PLL6 & 16.56 & $16.70 \%$ & $\mathrm{NO}$ & YES & $\mathrm{NO}$ & NO & YES \\
\hline 785 & O00232 & PSD12 & 52.91 & $8.55 \%$ & NO & $\mathrm{NO}$ & YES & NO & YES \\
\hline 786 & Q6WCQ1 & MPRIP & 116.54 & $3.80 \%$ & NO & NO & $\mathrm{NO}$ & $\mathrm{NO}$ & YES \\
\hline 787 & Q9BTE3 & MCMBP & 72.98 & $4.21 \%$ & NO & NO & $\mathrm{NO}$ & $\mathrm{NO}$ & YES \\
\hline 788 & Q13610 & PWP1 & 55.83 & $7.39 \%$ & NO & YES & $\mathrm{NO}$ & $\mathrm{NO}$ & YES \\
\hline 789 & Q9BQ52 & RNZ2 & 92.22 & $9.32 \%$ & NO & NO & NO & NO & YES \\
\hline 790 & P26196 & DDX6 & 54.42 & $7.45 \%$ & NO & NO & $\mathrm{NO}$ & $\mathrm{NO}$ & YES \\
\hline 791 & Q14738 & $2 \mathrm{~A} 5 \mathrm{D}$ & 69.99 & $3.49 \%$ & NO & NO & $\mathrm{NO}$ & $\mathrm{NO}$ & YES \\
\hline 792 & F6XY72 & F6XY72 & 8.67 & $79.20 \%$ & NO & YES & NO & NO & YES \\
\hline 793 & P63000 & $\mathrm{RAC1}$ & 21.45 & $12.00 \%$ & NO & $\mathrm{NO}$ & NO & NO & YES \\
\hline 794 & Q9UKV8 & $\mathrm{AGO} 2$ & 97.21 & $4.54 \%$ & NO & NO & $\mathrm{NO}$ & $\mathrm{NO}$ & YES \\
\hline 795 & O95347 & SMC2 & 135.66 & $4.51 \%$ & YES & NO & $\mathrm{NO}$ & $\mathrm{NO}$ & YES \\
\hline 796 & Q15021 & CND1 & 157.19 & $2.43 \%$ & YES & NO & $\mathrm{NO}$ & $\mathrm{NO}$ & YES \\
\hline 797 & Q86TU7 & SETD3 & 67.26 & $5.05 \%$ & NO & NO & NO & NO & YES \\
\hline 798 & B0UX83 & B0UX83 & 119.41 & $3.55 \%$ & NO & NO & $\mathrm{NO}$ & $\mathrm{NO}$ & YES \\
\hline 799 & O43396 & TXNL1 & 32.25 & $17.00 \%$ & NO & NO & YES & $\mathrm{NO}$ & YES \\
\hline 800 & Q9H488 & OFUT1 & 43.96 & $6.70 \%$ & NO & NO & $\mathrm{NO}$ & $\mathrm{NO}$ & YES \\
\hline 801 & Q03154 & ACY1 & 45.88 & $5.64 \%$ & NO & NO & YES & NO & YES \\
\hline 802 & B4DFK6 & B4DFK6 & 36.41 & $8.68 \%$ & NO & YES & $\mathrm{NO}$ & $\mathrm{NO}$ & YES \\
\hline 803 & P52735 & VAV2 & 101.29 & $3.08 \%$ & NO & NO & YES & $\mathrm{NO}$ & YES \\
\hline 804 & P62330 & ARF6 & 20.08 & $12.00 \%$ & NO & YES & YES & $\mathrm{NO}$ & YES \\
\hline 805 & O14737 & PDCD5 & 14.29 & $19.20 \%$ & YES & YES & $\mathrm{NO}$ & NO & YES \\
\hline 806 & Q8NE71 & ABCF1 & 95.93 & $7.57 \%$ & NO & $\mathrm{NO}$ & $\mathrm{NO}$ & $\mathrm{NO}$ & YES \\
\hline 807 & O14578 & CTRO & 231.44 & $1.48 \%$ & YES & $\mathrm{NO}$ & $\mathrm{NO}$ & NO & YES \\
\hline 808 & B4DW92 & B4DW92 & 15.88 & $11.40 \%$ & YES & YES & NO & NO & YES \\
\hline 809 & P07384 & CAN1 & 81.89 & $4.34 \%$ & NO & YES & YES & $\mathrm{NO}$ & YES \\
\hline 810 & P08123 & CO1A2 & 129.31 & $1.46 \%$ & NO & NO & YES & $\mathrm{NO}$ & YES \\
\hline 811 & Q13641 & TPBG & 46.03 & $5.71 \%$ & NO & NO & NO & $\mathrm{NO}$ & YES \\
\hline 812 & A8MU58 & A8MU58 & 35.35 & $9.92 \%$ & NO & YES & NO & NO & YES \\
\hline 813 & Q2NL82 & TSR1 & 91.81 & $3.48 \%$ & YES & $\mathrm{NO}$ & NO & $\mathrm{NO}$ & YES \\
\hline 814 & Q09161 & NCBP1 & 91.84 & $4.68 \%$ & NO & NO & NO & $\mathrm{NO}$ & YES \\
\hline 815 & O14497 & ARI1A & 68.04 & $1.14 \%$ & NO & $\mathrm{NO}$ & $\mathrm{NO}$ & NO & YES \\
\hline 816 & C9JPG5 & C9JPG5 & 88.38 & $2.92 \%$ & NO & NO & NO & NO & YES \\
\hline 817 & P49366 & DHYS & 40.97 & $10.00 \%$ & NO & YES & NO & $\mathrm{NO}$ & YES \\
\hline 818 & P41567 & EIF1 & 12.73 & $25.70 \%$ & NO & YES & NO & $\mathrm{NO}$ & YES \\
\hline 819 & C9JG87 & C9JG87 & 38.71 & $8.75 \%$ & NO & NO & $\mathrm{NO}$ & $\mathrm{NO}$ & YES \\
\hline 820 & C9J4M6 & C9J2Y9 & 133.90 & $1.89 \%$ & NO & NO & NO & NO & YES \\
\hline 821 & H0YLH3 & H0YLH3 & 65.07 & $7.31 \%$ & NO & NO & $\mathrm{NO}$ & $\mathrm{NO}$ & YES \\
\hline 822 & Q15056 & IF4H & 27.39 & $10.90 \%$ & NO & YES & YES & NO & YES \\
\hline 823 & P54886 & P5CS & 87.30 & $3.02 \%$ & NO & YES & NO & NO & YES \\
\hline 824 & P19875 & CXCL2 & 11.39 & $22.40 \%$ & NO & NO & NO & NO & YES \\
\hline 825 & Q9NQW6 & ANLN & 124.20 & $1.51 \%$ & NO & NO & $\mathrm{NO}$ & $\mathrm{NO}$ & YES \\
\hline 826 & E7EW00 & E7ET15 & 118.30 & $2.14 \%$ & NO & NO & $\mathrm{NO}$ & NO & YES \\
\hline 827 & B4DVN3 & B4DQT1 & 45.29 & $8.62 \%$ & NO & NO & NO & NO & YES \\
\hline 828 & E9PF63 & E9PF63 & 160.91 & $2.18 \%$ & NO & NO & NO & NO & YES \\
\hline 829 & O76061 & STC2 & 33.25 & $31.10 \%$ & YES & NO & $\mathrm{NO}$ & $\mathrm{NO}$ & YES \\
\hline 830 & F8VPD4 & F8VPD4 & 242.98 & $13.20 \%$ & NO & NO & $\mathrm{NO}$ & NO & YES \\
\hline 831 & H0Y6E7 & H0Y6E7 & 42.33 & $14.70 \%$ & YES & YES & $\mathrm{NO}$ & NO & YES \\
\hline 832 & B4DGP8 & B4DGP8 & 67.57 & $14.40 \%$ & NO & NO & $\mathrm{NO}$ & NO & YES \\
\hline 833 & F5H1A8 & F5H1A8 & 85.70 & $22.30 \%$ & NO & NO & $\mathrm{NO}$ & NO & YES \\
\hline
\end{tabular}




\begin{tabular}{|c|c|c|c|c|c|c|c|c|c|}
\hline 834 & P27695 & APEX1 & 35.56 & $24.20 \%$ & NO & YES & $\mathrm{NO}$ & $\mathrm{NO}$ & YES \\
\hline 835 & Q9Y376 & CAB39 & 39.87 & $17.90 \%$ & NO & YES & YES & $\mathrm{NO}$ & YES \\
\hline 836 & P35442 & TSP2 & 129.99 & $9.39 \%$ & $\mathrm{NO}$ & NO & YES & $\mathrm{NO}$ & YES \\
\hline 837 & P11766 & ADHX & 39.72 & $14.20 \%$ & $\mathrm{NO}$ & NO & YES & $\mathrm{NO}$ & YES \\
\hline 838 & Q8N163 & K1967 & 102.90 & $12.80 \%$ & $\mathrm{NO}$ & NO & NO & $\mathrm{NO}$ & YES \\
\hline 839 & P09661 & RU2A & 28.42 & $13.30 \%$ & $\mathrm{NO}$ & YES & NO & $\mathrm{NO}$ & YES \\
\hline 840 & P55809 & SCOT1 & 56.16 & $8.27 \%$ & $\mathrm{NO}$ & NO & YES & $\mathrm{NO}$ & YES \\
\hline 841 & Q99873 & ANM1 & 41.52 & $9.97 \%$ & $\mathrm{NO}$ & NO & NO & $\mathrm{NO}$ & YES \\
\hline 842 & Q06124 & PTN11 & 68.44 & $11.10 \%$ & $\mathrm{NO}$ & NO & NO & $\mathrm{NO}$ & YES \\
\hline 843 & P78347 & GTF2I & 112.42 & $2.20 \%$ & $\mathrm{NO}$ & NO & NO & $\mathrm{NO}$ & YES \\
\hline 844 & P04080 & CYTB & 11.14 & $45.90 \%$ & $\mathrm{NO}$ & NO & YES & $\mathrm{NO}$ & YES \\
\hline 845 & P05141 & ADT2 & 32.85 & $7.38 \%$ & $\mathrm{NO}$ & NO & YES & $\mathrm{NO}$ & YES \\
\hline 846 & P27694 & RFA1 & 68.14 & $7.63 \%$ & $\mathrm{NO}$ & NO & NO & $\mathrm{NO}$ & YES \\
\hline 847 & B4E2J3 & B4E2J3 & 39.64 & $11.40 \%$ & YES & NO & NO & $\mathrm{NO}$ & YES \\
\hline 848 & P61313 & RL15 & 24.15 & $24.50 \%$ & YES & NO & YES & $\mathrm{NO}$ & YES \\
\hline 849 & E7ESE0 & D6RAN4 & 21.86 & $20.30 \%$ & $\mathrm{NO}$ & YES & NO & $\mathrm{NO}$ & YES \\
\hline 850 & Q9BVP2 & GNL3 & 61.99 & $4.92 \%$ & $\mathrm{NO}$ & NO & NO & $\mathrm{NO}$ & YES \\
\hline 851 & Q9BZK7 & TBL1R & 55.59 & $8.37 \%$ & NO & YES & $\mathrm{NO}$ & $\mathrm{NO}$ & YES \\
\hline 852 & A8MW61 & A8MW61 & 57.19 & $3.50 \%$ & $\mathrm{NO}$ & NO & NO & $\mathrm{NO}$ & YES \\
\hline 853 & P68104 & EF1A1 & 50.14 & $53.70 \%$ & $\mathrm{NO}$ & NO & YES & $\mathrm{NO}$ & YES \\
\hline 854 & P11047 & LAMC1 & 177.60 & $27.20 \%$ & $\mathrm{NO}$ & NO & YES & $\mathrm{NO}$ & YES \\
\hline 855 & P09382 & LEG1 & 14.72 & $57.00 \%$ & $\mathrm{NO}$ & NO & YES & $\mathrm{NO}$ & YES \\
\hline 856 & Q12841 & FSTL1 & 34.99 & $45.80 \%$ & $\mathrm{NO}$ & NO & YES & $\mathrm{NO}$ & YES \\
\hline 857 & P34932 & HSP74 & 94.33 & $37.40 \%$ & NO & NO & YES & $\mathrm{NO}$ & YES \\
\hline 858 & P26022 & PTX3 & 41.98 & $34.40 \%$ & $\mathrm{NO}$ & NO & YES & $\mathrm{NO}$ & YES \\
\hline 859 & F5H025 & F5H025 & 140.00 & $24.90 \%$ & NO & NO & NO & $\mathrm{NO}$ & YES \\
\hline 860 & I3L404 & H0YEN5 & 31.33 & $48.20 \%$ & $\mathrm{NO}$ & YES & NO & $\mathrm{NO}$ & YES \\
\hline 861 & D6RAT0 & D6RAT0 & 29.95 & $27.80 \%$ & NO & YES & NO & $\mathrm{NO}$ & YES \\
\hline 862 & B7ZL00 & B7ZL00 & 133.02 & $8.40 \%$ & YES & $\mathrm{NO}$ & NO & $\mathrm{NO}$ & YES \\
\hline 863 & A6NG06 & A6NG06 & 22.09 & $55.30 \%$ & NO & YES & NO & $\mathrm{NO}$ & YES \\
\hline 864 & Q92520 & FAM3C & 24.68 & $30.40 \%$ & $\mathrm{NO}$ & $\mathrm{NO}$ & YES & $\mathrm{NO}$ & YES \\
\hline 865 & P46781 & RS9 & 22.59 & $22.20 \%$ & YES & NO & YES & $\mathrm{NO}$ & YES \\
\hline 866 & Q5VY30 & Q5VY30 & 23.01 & $30.70 \%$ & $\mathrm{NO}$ & YES & NO & $\mathrm{NO}$ & YES \\
\hline 867 & P21589 & 5NTD & 63.37 & $11.50 \%$ & YES & NO & NO & $\mathrm{NO}$ & YE \\
\hline 868 & Q13444 & ADA15 & 92.96 & $8.69 \%$ & YES & NO & NO & $\mathrm{NO}$ & YES \\
\hline 869 & A6NKQ9 & CGB1 & 20.47 & $19.80 \%$ & $\mathrm{NO}$ & NO & NO & $\mathrm{NO}$ & YES \\
\hline 870 & P55036 & PSMD4 & 40.74 & $21.00 \%$ & $\mathrm{NO}$ & NO & NO & $\mathrm{NO}$ & YES \\
\hline 871 & A8MXP8 & F8WCY5 & 36.88 & $11.90 \%$ & $\mathrm{NO}$ & NO & NO & $\mathrm{NO}$ & YES \\
\hline 872 & P48147 & PPCE & 80.70 & $10.60 \%$ & $\mathrm{NO}$ & YES & NO & $\mathrm{NO}$ & YES \\
\hline 873 & Q9NS15 & LTBP3 & 139.35 & $2.15 \%$ & $\mathrm{NO}$ & NO & NO & $\mathrm{NO}$ & YES \\
\hline 874 & G3V531 & F5H365 & 86.16 & $9.38 \%$ & $\mathrm{NO}$ & NO & $\mathrm{NO}$ & $\mathrm{NO}$ & YES \\
\hline 875 & E9PF18 & E9PF18 & 34.29 & $6.60 \%$ & $\mathrm{NO}$ & NO & NO & $\mathrm{NO}$ & YES \\
\hline 876 & G3V126 & G3V126 & 55.88 & $6.77 \%$ & YES & $\mathrm{NO}$ & NO & $\mathrm{NO}$ & YES \\
\hline 877 & B4E2W0 & B4E2W0 & 51.30 & $5.53 \%$ & $\mathrm{NO}$ & NO & NO & $\mathrm{NO}$ & YES \\
\hline 878 & P48729 & $\mathrm{KC} 1 \mathrm{~A}$ & 38.92 & $6.53 \%$ & NO & NO & YES & $\mathrm{NO}$ & YES \\
\hline 879 & D6RBW1 & D6RBW1 & 25.10 & $10.20 \%$ & $\mathrm{NO}$ & YES & NO & $\mathrm{NO}$ & YES \\
\hline 880 & B4DE16 & B4DE16 & 65.18 & $3.73 \%$ & $\mathrm{NO}$ & YES & NO & $\mathrm{NO}$ & YE \\
\hline 881 & H7C215 & $\mathrm{H} 7 \mathrm{C} 0 \mathrm{~V} 4$ & 50.71 & $4.87 \%$ & NO & NO & NO & $\mathrm{NO}$ & YES \\
\hline 882 & Q8TEQ6 & GEMI5 & 168.59 & $1.33 \%$ & YES & $\mathrm{NO}$ & NO & $\mathrm{NO}$ & YES \\
\hline 883 & P14618 & KPYM & 57.94 & $69.70 \%$ & YES & NO & YES & $\mathrm{NO}$ & $\mathrm{NO}$ \\
\hline 884 & Q08380 & LG3BP & 65.33 & $60.30 \%$ & $\mathrm{NO}$ & $\mathrm{NO}$ & YES & $\mathrm{NO}$ & $\mathrm{NO}$ \\
\hline 885 & P11142 & HSP7C & 70.90 & $59.90 \%$ & NO & NO & YES & $\mathrm{NO}$ & $\mathrm{NO}$ \\
\hline 886 & F8VYX6 & F8VW92 & 49.67 & $72.40 \%$ & NO & YES & NO & NO & NO \\
\hline 887 & P07355 & ANXA2 & 38.61 & $63.70 \%$ & NO & YES & YES & $\mathrm{NO}$ & $\mathrm{NO}$ \\
\hline 888 & P21333 & FLNA & 280.73 & $43.60 \%$ & $\mathrm{NO}$ & NO & YES & $\mathrm{NO}$ & $\mathrm{NO}$ \\
\hline 889 & P63104 & $1433 Z$ & 27.75 & $65.70 \%$ & YES & NO & YES & $\mathrm{NO}$ & $\mathrm{NO}$ \\
\hline
\end{tabular}




\begin{tabular}{|c|c|c|c|c|c|c|c|c|c|}
\hline 890 & O43707 & ACTN4 & 104.86 & $63.80 \%$ & NO & NO & YES & $\mathrm{NO}$ & NO \\
\hline 891 & P11021 & GRP78 & 72.33 & $52.80 \%$ & YES & NO & YES & $\mathrm{NO}$ & $\mathrm{NO}$ \\
\hline 892 & P55072 & TERA & 89.32 & $48.10 \%$ & NO & NO & YES & $\mathrm{NO}$ & $\mathrm{NO}$ \\
\hline 893 & F8VXB4 & F8VXB4 & 53.71 & $40.70 \%$ & NO & NO & NO & $\mathrm{NO}$ & $\mathrm{NO}$ \\
\hline 894 & P62937 & PPIA & 18.01 & $83.00 \%$ & NO & NO & YES & $\mathrm{NO}$ & $\mathrm{NO}$ \\
\hline 895 & O00391 & QSOX1 & 82.58 & $45.10 \%$ & YES & NO & YES & $\mathrm{NO}$ & $\mathrm{NO}$ \\
\hline 896 & Р07237 & PDIA1 & 57.12 & $51.80 \%$ & NO & NO & YES & $\mathrm{NO}$ & NO \\
\hline 897 & P09211 & GSTP1 & 23.36 & $89.50 \%$ & YES & YES & YES & $\mathrm{NO}$ & $\mathrm{NO}$ \\
\hline 898 & P22626 & ROA2 & 37.43 & $50.40 \%$ & YES & NO & YES & $\mathrm{NO}$ & $\mathrm{NO}$ \\
\hline 899 & P18206 & VINC & 123.80 & $36.30 \%$ & NO & NO & YES & $\mathrm{NO}$ & $\mathrm{NO}$ \\
\hline 900 & P13639 & EF2 & 95.34 & $47.60 \%$ & NO & NO & YES & $\mathrm{NO}$ & $\mathrm{NO}$ \\
\hline 901 & E7EPA7 & E7EPA7 & 67.88 & $60.90 \%$ & NO & NO & NO & $\mathrm{NO}$ & $\mathrm{NO}$ \\
\hline 902 & P02545 & LMNA & 74.14 & $38.10 \%$ & NO & NO & YES & $\mathrm{NO}$ & $\mathrm{NO}$ \\
\hline 903 & Q9Y490 & TLN1 & 269.77 & $29.00 \%$ & NO & NO & YES & $\mathrm{NO}$ & $\mathrm{NO}$ \\
\hline 904 & P49327 & FAS & 273.43 & $25.40 \%$ & YES & NO & YES & $\mathrm{NO}$ & NO \\
\hline 905 & P08238 & HS90B & 83.27 & $49.60 \%$ & YES & NO & YES & $\mathrm{NO}$ & NO \\
\hline 906 & A6NG51 & A6NG51 & 284.54 & $24.90 \%$ & NO & NO & NO & $\mathrm{NO}$ & $\mathrm{NO}$ \\
\hline 907 & P60842 & IF4A1 & 46.16 & $31.50 \%$ & YES & YES & YES & $\mathrm{NO}$ & $\mathrm{NO}$ \\
\hline 908 & P18669 & PGAM1 & 28.80 & $57.10 \%$ & YES & NO & YES & $\mathrm{NO}$ & $\mathrm{NO}$ \\
\hline 909 & P26038 & MOES & 67.82 & $36.90 \%$ & YES & YES & YES & $\mathrm{NO}$ & $\mathrm{NO}$ \\
\hline 910 & P40926 & MDHM & 35.50 & $56.20 \%$ & NO & YES & YES & $\mathrm{NO}$ & $\mathrm{NO}$ \\
\hline 911 & P61978 & HNRPK & 50.98 & $44.30 \%$ & YES & $\mathrm{NO}$ & YES & $\mathrm{NO}$ & $\mathrm{NO}$ \\
\hline 912 & Q6YHK3 & CD109 & 161.69 & $19.60 \%$ & NO & NO & YES & $\mathrm{NO}$ & NO \\
\hline 913 & P31939 & PUR9 & 64.62 & $48.00 \%$ & NO & NO & YES & $\mathrm{NO}$ & $\mathrm{NO}$ \\
\hline 914 & P22314 & UBA1 & 117.85 & $34.50 \%$ & NO & YES & YES & $\mathrm{NO}$ & $\mathrm{NO}$ \\
\hline 915 & Q32Q12 & Q32Q12 & 32.64 & $48.60 \%$ & NO & YES & YES & $\mathrm{NO}$ & $\mathrm{NO}$ \\
\hline 916 & P05783 & K1C18 & 48.06 & $50.20 \%$ & NO & YES & NO & $\mathrm{NO}$ & $\mathrm{NO}$ \\
\hline 917 & P05388 & RLA0 & 34.27 & $63.70 \%$ & YES & NO & YES & $\mathrm{NO}$ & $\mathrm{NO}$ \\
\hline 918 & E7EWK4 & H0Y8C6 & 123.63 & $23.70 \%$ & NO & YES & NO & $\mathrm{NO}$ & $\mathrm{NO}$ \\
\hline 919 & P48643 & TCPE & 59.67 & $48.80 \%$ & NO & NO & YES & $\mathrm{NO}$ & $\mathrm{NO}$ \\
\hline 920 & P14625 & ENPL & 92.47 & $29.80 \%$ & YES & NO & YES & $\mathrm{NO}$ & $\mathrm{NO}$ \\
\hline 921 & P13667 & PDIA4 & 72.93 & $28.20 \%$ & YES & NO & YES & $\mathrm{NO}$ & $\mathrm{NO}$ \\
\hline 922 & P30041 & PRDX6 & 25.04 & $57.60 \%$ & NO & NO & YES & $\mathrm{NO}$ & NO \\
\hline 923 & P08195 & $4 \mathrm{~F} 2$ & 68.00 & $21.70 \%$ & NO & YES & YES & $\mathrm{NO}$ & NO \\
\hline 924 & G5E9M5 & G5E9M5 & 95.34 & $22.20 \%$ & NO & NO & NO & $\mathrm{NO}$ & $\mathrm{NO}$ \\
\hline 925 & Q14697 & GANAB & 106.88 & $24.60 \%$ & NO & NO & YES & $\mathrm{NO}$ & $\mathrm{NO}$ \\
\hline 926 & Q5VU61 & Q5VU66 & 28.87 & $35.10 \%$ & YES & NO & NO & $\mathrm{NO}$ & $\mathrm{NO}$ \\
\hline 927 & G3V5Z7 & G3V5Z7 & 27.40 & $36.10 \%$ & NO & YES & NO & $\mathrm{NO}$ & NO \\
\hline 928 & D6R9D3 & G8JLB6 & 49.23 & $29.20 \%$ & NO & YES & NO & $\mathrm{NO}$ & $\mathrm{NO}$ \\
\hline 929 & P63010 & AP2B1 & 104.56 & $29.70 \%$ & YES & YES & NO & $\mathrm{NO}$ & $\mathrm{NO}$ \\
\hline 930 & P40227 & TCPZ & 58.03 & $36.90 \%$ & YES & NO & YES & NO & NO \\
\hline 931 & P11940 & PABP1 & 70.67 & $25.00 \%$ & YES & NO & YES & $\mathrm{NO}$ & NO \\
\hline 932 & B4DEM7 & B4DEM7 & 59.62 & $44.80 \%$ & NO & YES & NO & $\mathrm{NO}$ & $\mathrm{NO}$ \\
\hline 933 & P37837 & TALDO & 37.54 & $32.90 \%$ & NO & $\mathrm{NO}$ & YES & $\mathrm{NO}$ & $\mathrm{NO}$ \\
\hline 934 & E7EU23 & E7EU23 & 50.67 & $45.00 \%$ & NO & NO & NO & $\mathrm{NO}$ & $\mathrm{NO}$ \\
\hline 935 & H0YMA1 & H0YL69 & 29.48 & $51.70 \%$ & NO & YES & NO & $\mathrm{NO}$ & $\mathrm{NO}$ \\
\hline 936 & P53618 & COPB & 107.15 & $31.00 \%$ & NO & NO & YES & $\mathrm{NO}$ & NO \\
\hline 937 & Q9Y678 & COPG1 & 97.72 & $27.70 \%$ & YES & NO & YES & $\mathrm{NO}$ & $\mathrm{NO}$ \\
\hline 938 & H3BS10 & Н3ВР20 & 60.70 & $22.40 \%$ & NO & NO & NO & $\mathrm{NO}$ & $\mathrm{NO}$ \\
\hline 939 & P38646 & GRP75 & 73.68 & $24.20 \%$ & YES & NO & NO & $\mathrm{NO}$ & $\mathrm{NO}$ \\
\hline 940 & P15291 & B4GT1 & 43.92 & $28.40 \%$ & NO & YES & YES & $\mathrm{NO}$ & $\mathrm{NO}$ \\
\hline 941 & P13489 & RINI & 49.97 & $59.90 \%$ & YES & YES & YES & $\mathrm{NO}$ & $\mathrm{NO}$ \\
\hline 942 & Q9UBR2 & CATZ & 33.87 & $25.70 \%$ & NO & NO & YES & $\mathrm{NO}$ & NO \\
\hline 943 & B4DTC3 & B4DTC3 & 38.43 & $23.40 \%$ & NO & NO & NO & NO & $\mathrm{NO}$ \\
\hline 944 & P00491 & PNPH & 32.12 & $51.20 \%$ & YES & YES & YES & $\mathrm{NO}$ & $\mathrm{NO}$ \\
\hline 945 & P10586 & PTPRF & 212.88 & $13.70 \%$ & NO & NO & YES & $\mathrm{NO}$ & $\mathrm{NO}$ \\
\hline
\end{tabular}




\begin{tabular}{|c|c|c|c|c|c|c|c|c|}
\hline 946 & Q8WUM4 & PDC6I & 96.03 & $30.80 \%$ & $\mathrm{NO}$ & NO & YES & NO \\
\hline 947 & Q13200 & PSMD2 & 100.20 & $22.20 \%$ & YES & $\mathrm{NO}$ & YES & $\mathrm{NO}$ \\
\hline 948 & P63241 & IF5A1 & 16.83 & $44.20 \%$ & YES & NO & YES & $\mathrm{NO}$ \\
\hline 949 & P26006 & ITA3 & 116.61 & $14.80 \%$ & YES & NO & YES & NO \\
\hline 950 & B5MDF5 & B5MDF5 & 24.42 & $33.00 \%$ & $\mathrm{NO}$ & YES & NO & $\mathrm{NO}$ \\
\hline 951 & P17987 & TCPA & 60.35 & $48.20 \%$ & YES & YES & $\mathrm{NO}$ & $\mathrm{NO}$ \\
\hline 952 & P40121 & CAPG & 38.50 & $34.20 \%$ & $\mathrm{NO}$ & NO & YES & NO \\
\hline 953 & P50991 & TCPD & 57.93 & $34.90 \%$ & NO & YES & YES & NO \\
\hline 954 & P02786 & TFR1 & 84.87 & $26.80 \%$ & NO & YES & YES & $\mathrm{NO}$ \\
\hline 955 & E9PQZ1 & E9PRY8 & 31.12 & $15.80 \%$ & YES & NO & $\mathrm{NO}$ & $\mathrm{NO}$ \\
\hline 956 & A7MAP1 & A7MAP0 & 53.25 & $21.50 \%$ & NO & NO & $\mathrm{NO}$ & $\mathrm{NO}$ \\
\hline 957 & O75083 & WDR1 & 66.19 & $34.00 \%$ & NO & YES & YES & NO \\
\hline 958 & O95373 & IPO7 & 119.52 & $11.40 \%$ & YES & YES & $\mathrm{NO}$ & $\mathrm{NO}$ \\
\hline 959 & E7EWI9 & E7EWI9 & 39.60 & $30.40 \%$ & NO & NO & NO & $\mathrm{NO}$ \\
\hline 960 & O14979 & HNRDL & 46.44 & $21.00 \%$ & NO & NO & NO & $\mathrm{NO}$ \\
\hline 961 & P28070 & PSB4 & 29.21 & $35.60 \%$ & YES & YES & YES & NO \\
\hline 962 & P62333 & PRS10 & 44.17 & $28.80 \%$ & YES & YES & $\mathrm{NO}$ & $\mathrm{NO}$ \\
\hline 963 & P25398 & RS12 & 14.51 & $53.80 \%$ & NO & YES & YES & $\mathrm{NO}$ \\
\hline 964 & P48637 & GSHB & 52.39 & $28.70 \%$ & NO & NO & YES & $\mathrm{NO}$ \\
\hline 965 & Q9UNN8 & EPCR & 26.67 & $20.60 \%$ & NO & NO & YES & NO \\
\hline 966 & P16152 & CBR1 & 30.37 & $39.00 \%$ & NO & YES & YES & $\mathrm{NO}$ \\
\hline 967 & Q9UQ80 & PA2G4 & 43.79 & $42.10 \%$ & NO & NO & YES & $\mathrm{NO}$ \\
\hline 968 & P60981 & DEST & 18.51 & $43.00 \%$ & YES & YES & YES & $\mathrm{NO}$ \\
\hline 969 & P14866 & HNRPL & 64.13 & $19.20 \%$ & $\mathrm{NO}$ & NO & NO & $\mathrm{NO}$ \\
\hline 970 & B3KNT8 & B3KNT8 & 45.38 & $23.90 \%$ & $\mathrm{NO}$ & NO & NO & $\mathrm{NO}$ \\
\hline 971 & P23396 & RS3 & 26.69 & $45.70 \%$ & NO & NO & YES & $\mathrm{NO}$ \\
\hline 972 & P30050 & RL12 & 17.82 & $48.50 \%$ & NO & YES & YES & NO \\
\hline 973 & Q15365 & PCBP1 & 37.50 & $30.90 \%$ & $\mathrm{NO}$ & YES & YES & $\mathrm{NO}$ \\
\hline 974 & P49588 & SYAC & 106.81 & $13.20 \%$ & $\mathrm{NO}$ & NO & YES & $\mathrm{NO}$ \\
\hline 975 & P05023 & AT1A1 & 112.90 & $18.90 \%$ & NO & YES & YES & $\mathrm{NO}$ \\
\hline 976 & P62249 & RS16 & 16.45 & $39.70 \%$ & YES & YES & YES & $\mathrm{NO}$ \\
\hline 977 & E7ENR4 & E7ENR4 & 102.49 & $16.40 \%$ & $\mathrm{NO}$ & NO & NO & $\mathrm{NO}$ \\
\hline 978 & P04062 & GLCM & 59.72 & $25.40 \%$ & NO & NO & NO & NO \\
\hline 979 & B4DR31 & B4DR31 & 62.29 & $35.10 \%$ & $\mathrm{NO}$ & $\mathrm{NO}$ & $\mathrm{NO}$ & $\mathrm{NO}$ \\
\hline 980 & P52907 & CAZA1 & 32.92 & $39.20 \%$ & NO & NO & YES & NO \\
\hline 981 & P25788 & PSA3 & 28.43 & $28.20 \%$ & YES & NO & YES & $\mathrm{NO}$ \\
\hline 982 & P22102 & PUR2 & 107.77 & $21.50 \%$ & YES & YES & YES & $\mathrm{NO}$ \\
\hline 983 & P43686 & PRS6B & 47.37 & $16.50 \%$ & NO & YES & YES & $\mathrm{NO}$ \\
\hline 984 & O43505 & B3GN1 & 47.12 & $22.20 \%$ & YES & $\mathrm{NO}$ & YES & $\mathrm{NO}$ \\
\hline 985 & Q04917 & $1433 \mathrm{~F}$ & 28.22 & $27.20 \%$ & $\mathrm{NO}$ & NO & NO & $\mathrm{NO}$ \\
\hline 986 & E9PDC5 & E9PDC5 & 217.04 & $9.08 \%$ & NO & NO & NO & $\mathrm{NO}$ \\
\hline 987 & P30520 & PURA2 & 50.10 & $21.90 \%$ & YES & $\mathrm{NO}$ & NO & $\mathrm{NO}$ \\
\hline 988 & Q92945 & FUBP2 & 73.12 & $11.30 \%$ & YES & NO & NO & NO \\
\hline 989 & P62191 & PRS4 & 49.19 & $35.70 \%$ & $\mathrm{NO}$ & YES & $\mathrm{NO}$ & $\mathrm{NO}$ \\
\hline 990 & P13797 & PLST & 70.81 & $33.00 \%$ & NO & NO & YES & $\mathrm{NO}$ \\
\hline 991 & A6NHF2 & A6NHF2 & 61.33 & $16.30 \%$ & $\mathrm{NO}$ & YES & NO & NO \\
\hline 992 & A8K092 & A8K092 & 59.75 & $21.70 \%$ & YES & NO & NO & NO \\
\hline 993 & P49915 & GUAA & 76.72 & $25.70 \%$ & NO & NO & NO & $\mathrm{NO}$ \\
\hline 994 & P20618 & PSB1 & 26.49 & $54.80 \%$ & $\mathrm{NO}$ & NO & YES & NO \\
\hline 995 & E9PM69 & E9PM69 & 49.21 & $27.20 \%$ & $\mathrm{NO}$ & $\mathrm{NO}$ & NO & NO \\
\hline 996 & Q15437 & $\mathrm{SC} 23 \mathrm{~B}$ & 86.48 & $14.20 \%$ & $\mathrm{NO}$ & YES & NO & NO \\
\hline 997 & Q13263 & TIF1B & 88.55 & $16.50 \%$ & $\mathrm{NO}$ & $\mathrm{NO}$ & YES & $\mathrm{NO}$ \\
\hline 998 & F8VZJ2 & E9PAV3 & 205.42 & $3.37 \%$ & NO & YES & NO & $\mathrm{NO}$ \\
\hline 999 & P52272 & HNRPM & 77.52 & $17.80 \%$ & NO & NO & YES & $\mathrm{NO}$ \\
\hline 1000 & O15031 & PLXB2 & 205.13 & $10.30 \%$ & $\mathrm{NO}$ & $\mathrm{NO}$ & YES & $\mathrm{NO}$ \\
\hline 1001 & P00568 & KAD1 & 21.64 & $36.60 \%$ & YES & YES & YES & NO \\
\hline
\end{tabular}




\begin{tabular}{|c|c|c|c|c|c|c|c|c|c|}
\hline 1002 & P52788 & SPSY & 41.27 & $24.00 \%$ & $\mathrm{NO}$ & NO & $\mathrm{NO}$ & NO & $\mathrm{NO}$ \\
\hline 1003 & J3QQV5 & J3KTL2 & 27.75 & $20.90 \%$ & NO & YES & $\mathrm{NO}$ & $\mathrm{NO}$ & $\mathrm{NO}$ \\
\hline 1004 & P17900 & SAP3 & 20.84 & $29.00 \%$ & NO & $\mathrm{NO}$ & YES & NO & $\mathrm{NO}$ \\
\hline 1005 & E9PH29 & E9PH29 & 27.69 & $22.70 \%$ & YES & YES & $\mathrm{NO}$ & NO & $\mathrm{NO}$ \\
\hline 1006 & P54802 & ANAG & 82.27 & $20.20 \%$ & $\mathrm{NO}$ & NO & YES & NO & $\mathrm{NO}$ \\
\hline 1007 & Q07021 & C1QBP & 31.36 & $36.20 \%$ & YES & NO & $\mathrm{NO}$ & NO & $\mathrm{NO}$ \\
\hline 1008 & P35998 & PRS7 & 48.64 & $21.90 \%$ & YES & YES & $\mathrm{NO}$ & NO & $\mathrm{NO}$ \\
\hline 1009 & P28074 & PSB5 & 28.48 & $45.20 \%$ & YES & YES & YES & NO & $\mathrm{NO}$ \\
\hline 1010 & P56537 & IF6 & 26.60 & $41.20 \%$ & NO & YES & YES & NO & $\mathrm{NO}$ \\
\hline 1011 & P19623 & SPEE & 33.82 & $24.20 \%$ & YES & YES & $\mathrm{NO}$ & $\mathrm{NO}$ & $\mathrm{NO}$ \\
\hline 1012 & P07741 & APT & 19.61 & $40.00 \%$ & YES & NO & YES & NO & $\mathrm{NO}$ \\
\hline 1013 & P00533 & EGFR & 134.28 & $11.20 \%$ & NO & NO & YES & $\mathrm{NO}$ & $\mathrm{NO}$ \\
\hline 1014 & P62495 & ERF1 & 49.03 & $22.20 \%$ & NO & NO & $\mathrm{NO}$ & NO & $\mathrm{NO}$ \\
\hline 1015 & P23381 & SYWC & 53.17 & $13.80 \%$ & NO & NO & NO & NO & $\mathrm{NO}$ \\
\hline 1016 & P63167 & DYL1 & 10.37 & $46.10 \%$ & NO & NO & NO & NO & NO \\
\hline 1017 & P00492 & HPRT & 24.58 & $31.20 \%$ & YES & YES & YES & NO & $\mathrm{NO}$ \\
\hline 1018 & Q96EP5 & DAZP1 & 43.38 & $22.10 \%$ & NO & NO & NO & NO & $\mathrm{NO}$ \\
\hline 1019 & P30084 & ECHM & 31.39 & $21.70 \%$ & NO & YES & YES & $\mathrm{NO}$ & $\mathrm{NO}$ \\
\hline 1020 & P12429 & ANXA3 & 36.38 & $33.40 \%$ & NO & NO & YES & NO & $\mathrm{NO}$ \\
\hline 1021 & Q96AG4 & LRC59 & 34.93 & $28.00 \%$ & YES & YES & NO & NO & $\mathrm{NO}$ \\
\hline 1022 & B4DT69 & B4DT69 & 54.18 & $23.20 \%$ & $\mathrm{NO}$ & YES & NO & NO & $\mathrm{NO}$ \\
\hline 1023 & Q9H3G5 & CPVL & 54.17 & $18.90 \%$ & NO & NO & YES & NO & $\mathrm{NO}$ \\
\hline 1024 & O75534 & CSDE1 & 88.89 & $10.80 \%$ & NO & NO & NO & NO & $\mathrm{NO}$ \\
\hline 1025 & P45974 & UBP5 & 95.79 & $8.97 \%$ & NO & NO & YES & NO & $\mathrm{NO}$ \\
\hline 1026 & B4DHP2 & B4DP11 & 18.70 & $32.40 \%$ & NO & NO & NO & NO & $\mathrm{NO}$ \\
\hline 1027 & Q8NCH0 & CHSTE & 43.00 & $19.40 \%$ & NO & YES & $\mathrm{NO}$ & $\mathrm{NO}$ & $\mathrm{NO}$ \\
\hline 1028 & B4DLC0 & B4DLC0 & 38.58 & $23.90 \%$ & NO & YES & NO & NO & $\mathrm{NO}$ \\
\hline 1029 & B4E2V5 & B4E2V5 & 31.73 & $44.30 \%$ & NO & YES & NO & NO & $\mathrm{NO}$ \\
\hline 1030 & O15067 & PUR4 & 144.73 & $8.67 \%$ & NO & NO & NO & NO & $\mathrm{NO}$ \\
\hline 1031 & P21281 & VATB2 & 56.50 & $24.50 \%$ & NO & YES & YES & NO & NO \\
\hline 1032 & P02452 & CO1A1 & 138.94 & $2.80 \%$ & YES & NO & YES & NO & $\mathrm{NO}$ \\
\hline 1033 & P61160 & ARP2 & 44.76 & $22.60 \%$ & NO & NO & YES & NO & $\mathrm{NO}$ \\
\hline 1034 & O00505 & IMA3 & 57.81 & $23.60 \%$ & NO & YES & NO & $\mathrm{NO}$ & $\mathrm{NO}$ \\
\hline 1035 & F8W914 & F8W914 & 37.15 & $16.50 \%$ & NO & YES & $\mathrm{NO}$ & NO & $\mathrm{NO}$ \\
\hline 1036 & Q6NZI2 & PTRF & 43.48 & $11.30 \%$ & NO & YES & NO & NO & $\mathrm{NO}$ \\
\hline 1037 & Q9UL46 & PSME2 & 27.40 & $17.20 \%$ & NO & NO & YES & NO & $\mathrm{NO}$ \\
\hline 1038 & B4DJV2 & B4DJV2 & 51.71 & $14.10 \%$ & NO & YES & NO & NO & $\mathrm{NO}$ \\
\hline 1039 & Q02790 & FKBP4 & 51.81 & $17.40 \%$ & NO & NO & YES & NO & $\mathrm{NO}$ \\
\hline 1040 & P23919 & KTHY & 23.82 & $30.70 \%$ & YES & YES & NO & NO & $\mathrm{NO}$ \\
\hline 1041 & Q86UP2 & KTN1 & 156.28 & $7.37 \%$ & YES & NO & $\mathrm{NO}$ & NO & $\mathrm{NO}$ \\
\hline 1042 & Q09328 & MGT5A & 84.54 & $7.96 \%$ & $\mathrm{NO}$ & NO & YES & $\mathrm{NO}$ & $\mathrm{NO}$ \\
\hline 1043 & O43809 & CPSF5 & 26.23 & $16.30 \%$ & NO & NO & $\mathrm{NO}$ & NO & $\mathrm{NO}$ \\
\hline 1044 & Q9BTT0 & AN32E & 30.69 & $24.60 \%$ & NO & NO & NO & NO & $\mathrm{NO}$ \\
\hline 1045 & Q96HY6 & DDRGK & 35.61 & $15.60 \%$ & $\mathrm{NO}$ & NO & YES & NO & $\mathrm{NO}$ \\
\hline 1046 & B7Z1R5 & B7Z1R5 & 68.31 & $13.20 \%$ & NO & NO & NO & NO & $\mathrm{NO}$ \\
\hline 1047 & P34897 & GLYM & 55.99 & $14.50 \%$ & $\mathrm{NO}$ & NO & NO & NO & $\mathrm{NO}$ \\
\hline 1048 & H0Y4R1 & H0Y4R1 & 55.81 & $17.00 \%$ & YES & NO & NO & NO & $\mathrm{NO}$ \\
\hline 1049 & P10644 & KAP0 & 42.98 & $17.60 \%$ & $\mathrm{NO}$ & $\mathrm{NO}$ & $\mathrm{NO}$ & $\mathrm{NO}$ & $\mathrm{NO}$ \\
\hline 1050 & P61758 & PFD3 & 22.66 & $25.90 \%$ & YES & YES & $\mathrm{NO}$ & NO & $\mathrm{NO}$ \\
\hline 1051 & P61224 & RAP1B & 20.82 & $27.20 \%$ & $\mathrm{NO}$ & YES & NO & NO & $\mathrm{NO}$ \\
\hline 1052 & Q9UJJ9 & GNPTG & 33.97 & $23.00 \%$ & $\mathrm{NO}$ & NO & NO & NO & $\mathrm{NO}$ \\
\hline 1053 & Q6IBS0 & TWF2 & 39.55 & $26.40 \%$ & $\mathrm{NO}$ & NO & NO & NO & $\mathrm{NO}$ \\
\hline 1054 & P06132 & DCUP & 40.79 & $24.30 \%$ & $\mathrm{NO}$ & YES & $\mathrm{NO}$ & $\mathrm{NO}$ & $\mathrm{NO}$ \\
\hline 1055 & P61106 & RAB14 & 23.90 & $29.80 \%$ & NO & NO & YES & NO & $\mathrm{NO}$ \\
\hline 1056 & P62753 & RS6 & 28.68 & $17.70 \%$ & NO & $\mathrm{NO}$ & $\mathrm{NO}$ & $\mathrm{NO}$ & $\mathrm{NO}$ \\
\hline 1057 & P49773 & HINT1 & 13.80 & $40.50 \%$ & NO & YES & YES & $\mathrm{NO}$ & $\mathrm{NO}$ \\
\hline
\end{tabular}




\begin{tabular}{|c|c|c|c|c|c|c|c|c|c|}
\hline 1058 & J3KP15 & J3KP15 & 25.48 & $23.90 \%$ & NO & NO & NO & $\mathrm{NO}$ & NO \\
\hline 1059 & Q04837 & SSBP & 17.26 & $27.70 \%$ & YES & YES & $\mathrm{NO}$ & $\mathrm{NO}$ & $\mathrm{NO}$ \\
\hline 1060 & P28838 & AMPL & 56.17 & $19.80 \%$ & YES & NO & YES & $\mathrm{NO}$ & $\mathrm{NO}$ \\
\hline 1061 & O14579 & COPE & 34.48 & $30.80 \%$ & NO & NO & NO & $\mathrm{NO}$ & $\mathrm{NO}$ \\
\hline 1062 & Q15459 & SF3A1 & 88.89 & $10.20 \%$ & NO & NO & NO & $\mathrm{NO}$ & $\mathrm{NO}$ \\
\hline 1063 & A8MV58 & A8MV58 & 71.43 & $8.06 \%$ & NO & NO & $\mathrm{NO}$ & $\mathrm{NO}$ & $\mathrm{NO}$ \\
\hline 1064 & P38919 & IF4A3 & 46.87 & $29.90 \%$ & NO & YES & NO & $\mathrm{NO}$ & NO \\
\hline 1065 & Q5T4S7 & UBR4 & 573.84 & $2.72 \%$ & NO & NO & NO & $\mathrm{NO}$ & $\mathrm{NO}$ \\
\hline 1066 & E9PKL7 & E9PKL7 & 20.78 & $20.40 \%$ & NO & YES & NO & $\mathrm{NO}$ & $\mathrm{NO}$ \\
\hline 1067 & F2Z2Q5 & C9IZG4 & 19.12 & $23.70 \%$ & YES & YES & NO & $\mathrm{NO}$ & $\mathrm{NO}$ \\
\hline 1068 & P17812 & PYRG1 & 66.69 & $14.20 \%$ & YES & NO & NO & $\mathrm{NO}$ & NO \\
\hline 1069 & Q99598 & TSNAX & 33.11 & $35.50 \%$ & NO & NO & NO & $\mathrm{NO}$ & $\mathrm{NO}$ \\
\hline 1070 & P62424 & RL7A & 30.00 & $18.00 \%$ & NO & NO & NO & $\mathrm{NO}$ & $\mathrm{NO}$ \\
\hline 1071 & P31150 & GDIA & 50.58 & $27.70 \%$ & NO & NO & NO & NO & NO \\
\hline 1072 & P28062 & PSB8 & 30.35 & $21.70 \%$ & YES & YES & NO & $\mathrm{NO}$ & NO \\
\hline 1073 & B4E2Z2 & B4E2Z2 & 104.75 & $6.45 \%$ & NO & NO & NO & $\mathrm{NO}$ & NO \\
\hline 1074 & Q9Y3I0 & RTCB & 55.21 & $11.50 \%$ & YES & YES & NO & $\mathrm{NO}$ & $\mathrm{NO}$ \\
\hline 1075 & P78536 & ADA17 & 93.02 & $4.73 \%$ & NO & NO & NO & $\mathrm{NO}$ & $\mathrm{NO}$ \\
\hline 1076 & C9JJE2 & С9JJE2 & 68.33 & $6.53 \%$ & YES & NO & NO & $\mathrm{NO}$ & NO \\
\hline 1077 & A8MY36 & A8MY36 & 141.70 & $7.16 \%$ & NO & NO & NO & $\mathrm{NO}$ & $\mathrm{NO}$ \\
\hline 1078 & Q13347 & EIF3I & 36.50 & $20.30 \%$ & NO & YES & NO & $\mathrm{NO}$ & $\mathrm{NO}$ \\
\hline 1079 & Q5VZU9 & Q5VZU9 & 138.35 & $7.13 \%$ & YES & $\mathrm{NO}$ & NO & $\mathrm{NO}$ & $\mathrm{NO}$ \\
\hline 1080 & B4DVB8 & B4DVB8 & 36.09 & $18.70 \%$ & NO & YES & NO & $\mathrm{NO}$ & NO \\
\hline 1081 & A6NF51 & A6NF51 & 33.39 & $20.30 \%$ & NO & YES & NO & $\mathrm{NO}$ & $\mathrm{NO}$ \\
\hline 1082 & A8MTH6 & A8MTH6 & 21.68 & $21.90 \%$ & NO & YES & NO & $\mathrm{NO}$ & $\mathrm{NO}$ \\
\hline 1083 & Q03405 & UPAR & 36.98 & $17.00 \%$ & YES & NO & YES & NO & NO \\
\hline 1084 & Q10567 & AP1B1 & 104.64 & $25.30 \%$ & NO & NO & YES & $\mathrm{NO}$ & $\mathrm{NO}$ \\
\hline 1085 & P17655 & CAN2 & 80.00 & $14.90 \%$ & NO & YES & YES & $\mathrm{NO}$ & $\mathrm{NO}$ \\
\hline 1086 & F8WDG7 & E9PFZ5 & 118.39 & $9.28 \%$ & NO & $\mathrm{NO}$ & NO & $\mathrm{NO}$ & $\mathrm{NO}$ \\
\hline 1087 & F5H886 & F5H301 & 133.96 & $4.91 \%$ & YES & NO & NO & $\mathrm{NO}$ & $\mathrm{NO}$ \\
\hline 1088 & Q9Y266 & NUDC & 38.24 & $22.10 \%$ & NO & NO & NO & $\mathrm{NO}$ & $\mathrm{NO}$ \\
\hline 1089 & P33176 & KINH & 109.69 & $6.13 \%$ & NO & NO & YES & $\mathrm{NO}$ & $\mathrm{NO}$ \\
\hline 1090 & Q14444 & CAPR1 & 78.36 & $5.22 \%$ & YES & NO & NO & $\mathrm{NO}$ & NO \\
\hline 1091 & P20290 & BTF3 & 22.17 & $33.50 \%$ & NO & NO & NO & $\mathrm{NO}$ & NO \\
\hline 1092 & G5E9M3 & С9JAB2 & 27.37 & $14.90 \%$ & NO & YES & NO & $\mathrm{NO}$ & $\mathrm{NO}$ \\
\hline 1093 & P31949 & S10AB & 11.74 & $34.30 \%$ & NO & YES & YES & $\mathrm{NO}$ & $\mathrm{NO}$ \\
\hline 1094 & P21399 & ACOC & 98.40 & $15.40 \%$ & NO & $\mathrm{NO}$ & YES & $\mathrm{NO}$ & $\mathrm{NO}$ \\
\hline 1095 & O75533 & SF3B1 & 145.84 & $6.44 \%$ & NO & NO & NO & $\mathrm{NO}$ & NO \\
\hline 1096 & B7Z4B8 & B7Z4B8 & 95.74 & $9.26 \%$ & YES & $\mathrm{NO}$ & NO & $\mathrm{NO}$ & $\mathrm{NO}$ \\
\hline 1097 & E9PN11 & E9PQH6 & 22.01 & $37.30 \%$ & YES & YES & NO & $\mathrm{NO}$ & $\mathrm{NO}$ \\
\hline 1098 & Q15274 & NADC & 30.85 & $21.90 \%$ & YES & NO & YES & NO & NO \\
\hline 1099 & Q9H832 & UBE2Z & 38.21 & $4.52 \%$ & NO & YES & NO & $\mathrm{NO}$ & NO \\
\hline 1100 & O00487 & PSDE & 34.58 & $21.60 \%$ & NO & YES & NO & $\mathrm{NO}$ & $\mathrm{NO}$ \\
\hline 1101 & E9PKU4 & E9PKU4 & 28.02 & $26.00 \%$ & YES & YES & NO & $\mathrm{NO}$ & $\mathrm{NO}$ \\
\hline 1102 & B1AKZ3 & B1AKZ3 & 15.04 & $31.80 \%$ & NO & YES & NO & $\mathrm{NO}$ & $\mathrm{NO}$ \\
\hline 1103 & D6RB78 & D6RB78 & 63.94 & $5.92 \%$ & NO & $\mathrm{NO}$ & NO & $\mathrm{NO}$ & $\mathrm{NO}$ \\
\hline 1104 & Q14790 & CASP8 & 55.39 & $8.56 \%$ & NO & NO & NO & $\mathrm{NO}$ & NO \\
\hline 1105 & Q9BPX5 & ARP5L & 16.94 & $12.40 \%$ & NO & YES & YES & $\mathrm{NO}$ & $\mathrm{NO}$ \\
\hline 1106 & P30566 & PUR8 & 54.89 & $16.10 \%$ & NO & YES & NO & $\mathrm{NO}$ & $\mathrm{NO}$ \\
\hline 1107 & Q9UMX0 & UBQL1 & 62.52 & $14.80 \%$ & NO & NO & NO & $\mathrm{NO}$ & $\mathrm{NO}$ \\
\hline 1108 & $\mathrm{O} 43175$ & SERA & 56.65 & $12.80 \%$ & YES & NO & YES & $\mathrm{NO}$ & $\mathrm{NO}$ \\
\hline 1109 & Q9NPF2 & CHSTB & 41.56 & $15.90 \%$ & NO & NO & NO & $\mathrm{NO}$ & $\mathrm{NO}$ \\
\hline 1110 & Q07960 & RHG01 & 50.44 & $16.40 \%$ & NO & NO & YES & $\mathrm{NO}$ & NO \\
\hline 1111 & P50552 & VASP & 39.83 & $12.90 \%$ & NO & YES & NO & NO & $\mathrm{NO}$ \\
\hline 1112 & P11908 & PRPS2 & 34.77 & $12.90 \%$ & NO & NO & NO & $\mathrm{NO}$ & $\mathrm{NO}$ \\
\hline 1113 & P62873 & GBB1 & 37.38 & $35.60 \%$ & NO & NO & YES & $\mathrm{NO}$ & $\mathrm{NO}$ \\
\hline
\end{tabular}




\begin{tabular}{|c|c|c|c|c|c|c|c|c|}
\hline 1114 & Q9BX68 & HINT2 & 17.16 & $32.50 \%$ & YES & NO & NO & NO \\
\hline 1115 & P04183 & KITH & 25.47 & $15.80 \%$ & YES & YES & NO & $\mathrm{NO}$ \\
\hline 1116 & O00629 & IMA4 & 57.89 & $14.80 \%$ & YES & YES & NO & $\mathrm{NO}$ \\
\hline 1117 & P61026 & RAB10 & 22.54 & $20.00 \%$ & YES & NO & YES & $\mathrm{NO}$ \\
\hline 1118 & C9JQ42 & C9JQ42 & 21.51 & $9.02 \%$ & NO & YES & NO & NO \\
\hline 1119 & P36551 & HEM6 & 50.15 & $14.80 \%$ & NO & YES & NO & $\mathrm{NO}$ \\
\hline 1120 & Q12904 & AIMP1 & 34.35 & $17.30 \%$ & NO & YES & NO & $\mathrm{NO}$ \\
\hline 1121 & P49189 & AL9A1 & 53.80 & $9.11 \%$ & NO & NO & NO & NO \\
\hline 1122 & Q14703 & MBTP1 & 117.75 & $6.84 \%$ & YES & NO & YES & $\mathrm{NO}$ \\
\hline 1123 & Q01085 & TIAR & 41.59 & $13.10 \%$ & NO & NO & NO & $\mathrm{NO}$ \\
\hline 1124 & B4DLW8 & B4DLW8 & 69.15 & $15.10 \%$ & NO & YES & NO & $\mathrm{NO}$ \\
\hline 1125 & P53004 & BIEA & 33.43 & $12.50 \%$ & NO & YES & YES & $\mathrm{NO}$ \\
\hline 1126 & Q9Y696 & CLIC4 & 28.77 & $25.70 \%$ & NO & YES & YES & $\mathrm{NO}$ \\
\hline 1127 & P49746 & TSP3 & 104.20 & $9.21 \%$ & NO & NO & NO & NO \\
\hline 1128 & J3KPM9 & J3KPM9 & 87.34 & $9.52 \%$ & NO & NO & NO & $\mathrm{NO}$ \\
\hline 1129 & B4DZM8 & B4DZM8 & 56.20 & $16.10 \%$ & YES & YES & NO & NO \\
\hline 1130 & A8MV37 & A8MV37 & 35.54 & $25.30 \%$ & YES & YES & NO & $\mathrm{NO}$ \\
\hline 1131 & J3KPM5 & J3KPM5 & 13.53 & $35.20 \%$ & NO & YES & NO & $\mathrm{NO}$ \\
\hline 1132 & P68032 & ACTC & 42.02 & $41.60 \%$ & NO & YES & NO & $\mathrm{NO}$ \\
\hline 1133 & G5E9V1 & G5E9V1 & 43.45 & $15.40 \%$ & YES & NO & NO & NO \\
\hline 1134 & Q16630 & CPSF6 & 59.21 & $12.50 \%$ & NO & NO & NO & $\mathrm{NO}$ \\
\hline 1135 & Q8NDH3 & PEPL1 & 55.86 & $10.70 \%$ & YES & NO & NO & NO \\
\hline 1136 & Q01459 & DIAC & 43.76 & $14.50 \%$ & YES & NO & YES & $\mathrm{NO}$ \\
\hline 1137 & B4DVE7 & ANX11 & 54.39 & $9.96 \%$ & YES & YES & NO & NO \\
\hline 1138 & Q9NR31 & SAR1A & 22.37 & $33.30 \%$ & YES & YES & YES & $\mathrm{NO}$ \\
\hline 1139 & P48556 & PSMD8 & 39.61 & $14.60 \%$ & NO & YES & NO & $\mathrm{NO}$ \\
\hline 1140 & O00148 & DX39A & 49.13 & $29.30 \%$ & NO & YES & YES & NO \\
\hline 1141 & B0V0T3 & A2ACR1 & 23.26 & $24.50 \%$ & NO & YES & NO & NO \\
\hline 1142 & P34096 & RNAS4 & 16.84 & $17.00 \%$ & NO & NO & YES & $\mathrm{NO}$ \\
\hline 1143 & Q9H773 & DCTP1 & 18.68 & $16.50 \%$ & NO & YES & NO & NO \\
\hline 1144 & P46926 & GNPI1 & 32.67 & $24.20 \%$ & NO & NO & YES & NO \\
\hline 1145 & Q86VR8 & FJX1 & 48.51 & $13.50 \%$ & NO & NO & NO & NO \\
\hline 1146 & P34896 & GLYC & 53.08 & $12.40 \%$ & $\mathrm{NO}$ & NO & YES & $\mathrm{NO}$ \\
\hline 1147 & Q9BQG0 & MBB1A & 148.86 & $3.92 \%$ & NO & NO & NO & $\mathrm{NO}$ \\
\hline 1148 & Q9BQ67 & GRWD1 & 49.42 & $12.30 \%$ & NO & YES & NO & NO \\
\hline 1149 & P52888 & THOP1 & 78.84 & $4.50 \%$ & NO & NO & NO & $\mathrm{NO}$ \\
\hline 1150 & Q01581 & HMCS1 & 57.30 & $6.35 \%$ & YES & YES & NO & NO \\
\hline 1151 & P00734 & THRB & 70.04 & $4.02 \%$ & NO & NO & YES & $\mathrm{NO}$ \\
\hline 1152 & Q96IU4 & ABHEB & 22.35 & $17.60 \%$ & NO & YES & YES & NO \\
\hline 1153 & E9PPG9 & E9PQ57 & 40.97 & $9.38 \%$ & NO & YES & NO & NO \\
\hline 1154 & Q9H0C8 & ILKAP & 42.91 & $8.93 \%$ & $\mathrm{NO}$ & YES & NO & $\mathrm{NO}$ \\
\hline 1155 & Q9H008 & LHPP & 29.17 & $20.40 \%$ & NO & NO & YES & $\mathrm{NO}$ \\
\hline 1156 & P53999 & ТCP4 & 14.40 & $17.30 \%$ & NO & YES & NO & NO \\
\hline 1157 & P09012 & SNRPA & 31.28 & $13.10 \%$ & NO & YES & NO & $\mathrm{NO}$ \\
\hline 1158 & Q9UBS4 & DJB11 & 40.51 & $10.90 \%$ & NO & NO & NO & $\mathrm{NO}$ \\
\hline 1159 & F2Z2U8 & F2Z2U8 & 227.87 & $4.24 \%$ & NO & NO & NO & $\mathrm{NO}$ \\
\hline 1160 & Q8WUW1 & BRK1 & 8.75 & $32.00 \%$ & NO & YES & NO & NO \\
\hline 1161 & Q9UHV9 & PFD2 & 16.65 & $16.90 \%$ & NO & YES & NO & $\mathrm{NO}$ \\
\hline 1162 & Q6UXH8 & CCBE1 & 44.10 & $5.67 \%$ & NO & NO & NO & $\mathrm{NO}$ \\
\hline 1163 & Q9Y5L4 & TIM13 & 10.50 & $25.30 \%$ & NO & YES & NO & $\mathrm{NO}$ \\
\hline 1164 & P61201 & CSN2 & 51.60 & $10.60 \%$ & YES & NO & NO & $\mathrm{NO}$ \\
\hline 1165 & P67870 & CSK2B & 24.94 & $28.80 \%$ & NO & YES & NO & $\mathrm{NO}$ \\
\hline 1166 & Q00534 & CDK6 & 36.94 & $7.36 \%$ & YES & NO & $\mathrm{NO}$ & $\mathrm{NO}$ \\
\hline 1167 & Q96CT7 & CC124 & 25.84 & $15.70 \%$ & NO & NO & NO & NO \\
\hline 1168 & P24666 & PPAC & 18.04 & $20.90 \%$ & $\mathrm{NO}$ & YES & YES & $\mathrm{NO}$ \\
\hline 1169 & B1AKC9 & B1AKC9 & 117.49 & $5.29 \%$ & NO & NO & NO & $\mathrm{NO}$ \\
\hline
\end{tabular}




\begin{tabular}{|c|c|c|c|c|c|c|c|c|}
\hline 1170 & J3KMW6 & I3L2T3 & 31.21 & $18.90 \%$ & YES & YES & NO & NO \\
\hline 1171 & Q13509 & TBB3 & 50.43 & $38.70 \%$ & YES & NO & NO & $\mathrm{NO}$ \\
\hline 1172 & P62995 & TRA2B & 33.67 & $12.20 \%$ & $\mathrm{NO}$ & NO & NO & NO \\
\hline 1173 & P13804 & ETFA & 35.08 & $8.71 \%$ & NO & NO & YES & NO \\
\hline 1174 & P13987 & CD59 & 14.18 & $15.60 \%$ & $\mathrm{NO}$ & $\mathrm{NO}$ & YES & $\mathrm{NO}$ \\
\hline 1175 & O95967 & FBLN4 & 49.40 & $5.64 \%$ & $\mathrm{NO}$ & $\mathrm{NO}$ & YES & NO \\
\hline 1176 & D6REA1 & D6REA1 & 52.09 & $4.27 \%$ & NO & NO & NO & NO \\
\hline 1177 & Q7L2H7 & EIF3M & 42.50 & $18.70 \%$ & YES & NO & NO & NO \\
\hline 1178 & Q8TAT6 & NPL4 & 68.12 & $10.40 \%$ & NO & YES & NO & NO \\
\hline 1179 & O43286 & B4GT5 & 45.12 & $13.40 \%$ & $\mathrm{NO}$ & NO & NO & NO \\
\hline 1180 & O95831 & AIFM1 & 66.90 & $10.10 \%$ & $\mathrm{NO}$ & $\mathrm{NO}$ & NO & $\mathrm{NO}$ \\
\hline 1181 & $\mathrm{O} 43684$ & BUB3 & 37.15 & $20.40 \%$ & NO & YES & NO & NO \\
\hline 1182 & O00264 & PGRC1 & 21.67 & $16.40 \%$ & NO & NO & YES & NO \\
\hline 1183 & Q96AY3 & FKB10 & 64.25 & $9.11 \%$ & $\mathrm{NO}$ & NO & NO & NO \\
\hline 1184 & P43487 & RANG & 23.31 & $21.40 \%$ & YES & YES & YES & NO \\
\hline 1185 & P07225 & PROS & 75.12 & $5.92 \%$ & NO & NO & YES & NO \\
\hline 1186 & C9JIZ0 & C9JIZ0 & 10.40 & $78.70 \%$ & NO & YES & NO & NO \\
\hline 1187 & Q5T5C7 & Q5T5C7 & 58.78 & $3.17 \%$ & YES & NO & NO & NO \\
\hline 1188 & F5H4C2 & F5H4C2 & 60.21 & $7.29 \%$ & NO & NO & NO & NO \\
\hline 1189 & E9PCI9 & E9PCI9 & 48.28 & $21.00 \%$ & NO & NO & NO & NO \\
\hline 1190 & C9JV77 & C9JV77 & 39.32 & $5.16 \%$ & YES & $\mathrm{NO}$ & NO & $\mathrm{NO}$ \\
\hline 1191 & P84090 & ERH & 12.26 & $32.70 \%$ & $\mathrm{NO}$ & YES & NO & NO \\
\hline 1192 & P59998 & ARPC4 & 19.67 & $17.90 \%$ & NO & YES & YES & NO \\
\hline 1193 & Q96HE7 & ERO1A & 54.39 & $11.80 \%$ & $\mathrm{NO}$ & NO & YES & $\mathrm{NO}$ \\
\hline 1194 & P26572 & MGAT1 & 50.88 & $12.10 \%$ & NO & NO & NO & $\mathrm{NO}$ \\
\hline 1195 & Q96RS6 & NUDC1 & 66.76 & $9.43 \%$ & $\mathrm{NO}$ & NO & NO & $\mathrm{NO}$ \\
\hline 1196 & Р50281 & MMP14 & 65.90 & $7.39 \%$ & NO & NO & NO & NO \\
\hline 1197 & $\mathrm{O} 43172$ & PRP4 & 58.45 & $9.58 \%$ & $\mathrm{NO}$ & NO & NO & $\mathrm{NO}$ \\
\hline 1198 & P17405 & ASM & 69.75 & $8.90 \%$ & NO & YES & YES & $\mathrm{NO}$ \\
\hline 1199 & Q9Y315 & DEOC & 35.23 & $15.70 \%$ & $\mathrm{NO}$ & NO & NO & $\mathrm{NO}$ \\
\hline 1200 & B4DHT4 & B4DHT4 & 65.38 & $13.40 \%$ & YES & NO & NO & NO \\
\hline 1201 & Q9UBE0 & SAE1 & 38.45 & $11.60 \%$ & NO & YES & NO & NO \\
\hline 1202 & P36405 & ARL3 & 20.46 & $27.50 \%$ & $\mathrm{NO}$ & YES & YES & NO \\
\hline 1203 & P10768 & ESTD & 31.46 & $18.10 \%$ & NO & YES & YES & NO \\
\hline 1204 & Q15024 & EXOS7 & 31.82 & $17.90 \%$ & NO & NO & NO & NO \\
\hline 1205 & B4DWK0 & D6PSQ9 & 42.91 & $15.10 \%$ & YES & YES & NO & $\mathrm{NO}$ \\
\hline 1206 & E9PC52 & E9PC52 & 47.82 & $25.70 \%$ & $\mathrm{NO}$ & YES & NO & NO \\
\hline 1207 & Q15907 & RB11B & 24.49 & $11.90 \%$ & NO & YES & YES & $\mathrm{NO}$ \\
\hline 1208 & P11388 & TOP2A & 174.39 & $2.09 \%$ & NO & NO & NO & NO \\
\hline 1209 & O15116 & LSM1 & 15.18 & $16.50 \%$ & YES & $\mathrm{NO}$ & NO & $\mathrm{NO}$ \\
\hline 1210 & B1ANR0 & B1ANR0 & 70.78 & $20.20 \%$ & $\mathrm{NO}$ & NO & NO & NO \\
\hline 1211 & F8VZW6 & F8VTJ5 & 126.93 & $4.95 \%$ & NO & YES & NO & NO \\
\hline 1212 & Q9UI30 & TR112 & 14.20 & $44.80 \%$ & $\mathrm{NO}$ & YES & NO & NO \\
\hline 1213 & B7Z1G9 & B7Z6H3 & 69.16 & $10.60 \%$ & $\mathrm{NO}$ & YES & NO & NO \\
\hline 1214 & P20042 & IF2B & 38.39 & $7.21 \%$ & $\mathrm{NO}$ & NO & YES & $\mathrm{NO}$ \\
\hline 1215 & Q9Y3A5 & SBDS & 28.76 & $11.20 \%$ & NO & YES & NO & NO \\
\hline 1216 & Q9H0W9 & CK054 & 35.12 & $12.70 \%$ & $\mathrm{NO}$ & NO & YES & $\mathrm{NO}$ \\
\hline 1217 & Q9UKK3 & PARP4 & 192.60 & $2.26 \%$ & $\mathrm{NO}$ & NO & NO & NO \\
\hline 1218 & B7Z5Z2 & B7Z5Z2 & 23.40 & $18.60 \%$ & $\mathrm{NO}$ & YES & NO & NO \\
\hline 1219 & Q9HCD5 & NCOA5 & 65.54 & $8.64 \%$ & NO & NO & NO & NO \\
\hline 1220 & Q14258 & TRI25 & 70.97 & $5.71 \%$ & NO & NO & NO & NO \\
\hline 1221 & Q92890 & UFD1 & 34.50 & $7.49 \%$ & $\mathrm{NO}$ & YES & NO & NO \\
\hline 1222 & Q14320 & FA50A & 40.24 & $9.44 \%$ & $\mathrm{NO}$ & NO & NO & NO \\
\hline 1223 & Q13242 & SRSF9 & 25.54 & $17.20 \%$ & $\mathrm{NO}$ & NO & NO & $\mathrm{NO}$ \\
\hline 1224 & F8WAA0 & E7ENG9 & 165.68 & $2.46 \%$ & $\mathrm{NO}$ & YES & NO & NO \\
\hline 1225 & F6SBX2 & F6SBX2 & 113.79 & $2.66 \%$ & NO & NO & NO & NO \\
\hline
\end{tabular}




\begin{tabular}{|c|c|c|c|c|c|c|c|c|c|}
\hline 1226 & O00273 & DFFA & 36.52 & $8.76 \%$ & NO & NO & $\mathrm{NO}$ & NO & $\mathrm{NO}$ \\
\hline 1227 & G3V1L9 & G3V1L9 & 195.46 & $1.13 \%$ & $\mathrm{NO}$ & NO & $\mathrm{NO}$ & $\mathrm{NO}$ & $\mathrm{NO}$ \\
\hline 1228 & Q9BU89 & DOHH & 32.90 & $6.29 \%$ & NO & YES & $\mathrm{NO}$ & NO & $\mathrm{NO}$ \\
\hline 1229 & C9J8U2 & С9JC60 & 57.58 & $17.50 \%$ & YES & YES & $\mathrm{NO}$ & NO & $\mathrm{NO}$ \\
\hline 1230 & P50579 & AMPM2 & 52.89 & $12.10 \%$ & NO & NO & $\mathrm{NO}$ & $\mathrm{NO}$ & $\mathrm{NO}$ \\
\hline 1231 & F5H468 & E7EMD0 & 76.69 & $9.33 \%$ & NO & YES & $\mathrm{NO}$ & NO & $\mathrm{NO}$ \\
\hline 1232 & Q96J02 & ITCH & 102.81 & $6.42 \%$ & YES & NO & YES & NO & $\mathrm{NO}$ \\
\hline 1233 & P48163 & MAOX & 64.15 & $9.62 \%$ & NO & NO & YES & NO & $\mathrm{NO}$ \\
\hline 1234 & O14617 & AP3D1 & 130.16 & $5.46 \%$ & NO & YES & $\mathrm{NO}$ & NO & $\mathrm{NO}$ \\
\hline 1235 & P15170 & ERF3A & 55.76 & $8.02 \%$ & NO & $\mathrm{NO}$ & $\mathrm{NO}$ & $\mathrm{NO}$ & $\mathrm{NO}$ \\
\hline 1236 & O00170 & AIP & 37.64 & $13.60 \%$ & NO & NO & $\mathrm{NO}$ & NO & $\mathrm{NO}$ \\
\hline 1237 & Q16186 & ADRM1 & 42.15 & $9.09 \%$ & NO & NO & YES & NO & $\mathrm{NO}$ \\
\hline 1238 & P29218 & IMPA1 & 30.19 & $12.30 \%$ & NO & YES & YES & NO & $\mathrm{NO}$ \\
\hline 1239 & Q6UY14 & ATL4 & 116.54 & $2.61 \%$ & NO & NO & YES & NO & $\mathrm{NO}$ \\
\hline 1240 & Q9H223 & EHD4 & 61.18 & $5.91 \%$ & NO & NO & YES & NO & NO \\
\hline 1241 & B4DUA9 & B4DQI6 & 32.69 & $16.70 \%$ & NO & NO & NO & NO & $\mathrm{NO}$ \\
\hline 1242 & Q9GZT8 & NIF3L & 41.97 & $7.16 \%$ & NO & YES & NO & NO & $\mathrm{NO}$ \\
\hline 1243 & А8MXH2 & A $8 \mathrm{MXH} 2$ & 42.82 & $25.00 \%$ & NO & YES & $\mathrm{NO}$ & $\mathrm{NO}$ & $\mathrm{NO}$ \\
\hline 1244 & P27701 & CD82 & 29.63 & $11.20 \%$ & NO & NO & YES & NO & $\mathrm{NO}$ \\
\hline 1245 & Q5JU69 & TOR2A & 35.71 & $5.61 \%$ & NO & NO & NO & NO & $\mathrm{NO}$ \\
\hline 1246 & E9PGT1 & E9PGT1 & 26.18 & $13.90 \%$ & YES & NO & NO & NO & $\mathrm{NO}$ \\
\hline 1247 & P61163 & ACTZ & 42.62 & $20.70 \%$ & YES & NO & NO & NO & $\mathrm{NO}$ \\
\hline 1248 & Q9NQT4 & EXOS5 & 25.25 & $21.30 \%$ & NO & NO & NO & NO & $\mathrm{NO}$ \\
\hline 1249 & Q9H2M9 & RBGPR & 155.99 & $2.30 \%$ & YES & NO & NO & NO & $\mathrm{NO}$ \\
\hline 1250 & P30740 & ILEU & 42.74 & $11.90 \%$ & NO & YES & YES & NO & $\mathrm{NO}$ \\
\hline 1251 & Q13043 & STK4 & 55.63 & $7.80 \%$ & NO & NO & $\mathrm{NO}$ & $\mathrm{NO}$ & $\mathrm{NO}$ \\
\hline 1252 & Q12933 & TRAF2 & 55.86 & $6.19 \%$ & NO & NO & NO & NO & $\mathrm{NO}$ \\
\hline 1253 & P00918 & CAH2 & 29.25 & $16.90 \%$ & NO & NO & YES & NO & $\mathrm{NO}$ \\
\hline 1254 & B4DN22 & B4DN22 & 27.62 & $15.70 \%$ & NO & YES & NO & NO & $\mathrm{NO}$ \\
\hline 1255 & F5H345 & F5H345 & 39.33 & $6.36 \%$ & YES & NO & NO & NO & $\mathrm{NO}$ \\
\hline 1256 & I3L3C4 & I3L3U9 & 54.97 & $12.50 \%$ & NO & YES & NO & NO & $\mathrm{NO}$ \\
\hline 1257 & E7ESP4 & E7ESP4 & 129.30 & $3.61 \%$ & NO & NO & $\mathrm{NO}$ & NO & $\mathrm{NO}$ \\
\hline 1258 & C9J1U4 & C9J1U4 & 34.08 & $11.30 \%$ & NO & YES & NO & $\mathrm{NO}$ & $\mathrm{NO}$ \\
\hline 1259 & Q9UQB8 & BAIP2 & 60.87 & $4.89 \%$ & NO & NO & YES & NO & $\mathrm{NO}$ \\
\hline 1260 & P14550 & AK1A1 & 36.57 & $11.10 \%$ & NO & NO & YES & NO & $\mathrm{NO}$ \\
\hline 1261 & E9PAY3 & E9PAY3 & 21.88 & $18.00 \%$ & YES & YES & $\mathrm{NO}$ & NO & $\mathrm{NO}$ \\
\hline 1262 & O75915 & PRAF3 & 21.62 & $16.00 \%$ & NO & YES & NO & NO & $\mathrm{NO}$ \\
\hline 1263 & P12259 & FA5 & 251.71 & $1.08 \%$ & NO & NO & YES & NO & $\mathrm{NO}$ \\
\hline 1264 & P46779 & RL28 & 15.75 & $10.90 \%$ & NO & YES & NO & NO & $\mathrm{NO}$ \\
\hline 1265 & O60664 & PLIN3 & 47.07 & $13.10 \%$ & NO & YES & YES & NO & $\mathrm{NO}$ \\
\hline 1266 & Q12913 & PTPRJ & 145.94 & $4.41 \%$ & NO & NO & YES & $\mathrm{NO}$ & $\mathrm{NO}$ \\
\hline 1267 & A6NCK0 & A6NCK0 & 60.25 & $10.20 \%$ & NO & YES & $\mathrm{NO}$ & NO & $\mathrm{NO}$ \\
\hline 1268 & E7EM71 & E7EM71 & 141.46 & $3.72 \%$ & NO & NO & $\mathrm{NO}$ & NO & $\mathrm{NO}$ \\
\hline 1269 & P49902 & $5 \mathrm{NTC}$ & 64.97 & $7.66 \%$ & NO & NO & YES & NO & $\mathrm{NO}$ \\
\hline 1270 & P58397 & ATS12 & 177.67 & $2.57 \%$ & NO & NO & NO & NO & $\mathrm{NO}$ \\
\hline 1271 & Q9UJW0 & DCTN4 & 52.34 & $8.48 \%$ & NO & NO & NO & NO & $\mathrm{NO}$ \\
\hline 1272 & Q9HAV7 & GRPE1 & 24.28 & $14.30 \%$ & NO & NO & NO & NO & $\mathrm{NO}$ \\
\hline 1273 & P45973 & CBX5 & 22.23 & $21.50 \%$ & NO & YES & $\mathrm{NO}$ & $\mathrm{NO}$ & $\mathrm{NO}$ \\
\hline 1274 & B4DZW6 & B4DZW6 & 35.81 & $6.34 \%$ & NO & NO & $\mathrm{NO}$ & NO & $\mathrm{NO}$ \\
\hline 1275 & D6RE95 & E9PH06 & 66.32 & $5.23 \%$ & NO & YES & NO & NO & $\mathrm{NO}$ \\
\hline 1276 & Q9NXH8 & TOR4A & 46.91 & $6.38 \%$ & YES & YES & $\mathrm{NO}$ & NO & $\mathrm{NO}$ \\
\hline 1277 & P42771 & CD2A1 & 16.53 & $15.40 \%$ & NO & YES & NO & NO & $\mathrm{NO}$ \\
\hline 1278 & H0YMF9 & H0YL19 & 33.58 & $16.00 \%$ & NO & YES & $\mathrm{NO}$ & NO & $\mathrm{NO}$ \\
\hline 1279 & Q9P260 & K1468 & 134.63 & $2.38 \%$ & NO & NO & NO & NO & $\mathrm{NO}$ \\
\hline 1280 & P17050 & NAGAB & 46.57 & $6.57 \%$ & YES & NO & YES & NO & $\mathrm{NO}$ \\
\hline 1281 & P49247 & RPIA & 33.27 & $10.60 \%$ & NO & YES & $\mathrm{NO}$ & $\mathrm{NO}$ & $\mathrm{NO}$ \\
\hline
\end{tabular}




\begin{tabular}{|c|c|c|c|c|c|c|c|c|}
\hline 1282 & Q53H96 & P5CR3 & 28.65 & $10.60 \%$ & NO & YES & NO & $\mathrm{NO}$ \\
\hline 1283 & F8WEB5 & A6NK56 & 60.54 & $5.87 \%$ & NO & NO & NO & NO \\
\hline 1284 & Q9NPD3 & EXOS4 & 26.38 & $10.60 \%$ & NO & NO & NO & NO \\
\hline 1285 & B4E016 & B4E016 & 24.93 & $13.10 \%$ & NO & YES & NO & NO \\
\hline 1286 & O43765 & SGTA & 34.06 & $7.99 \%$ & NO & YES & NO & NO \\
\hline 1287 & P58107 & EPIPL & 555.62 & $3.01 \%$ & YES & $\mathrm{NO}$ & NO & NO \\
\hline 1288 & B1AH89 & B1AH89 & 74.40 & $8.81 \%$ & NO & YES & NO & NO \\
\hline 1289 & O95372 & LYPA2 & 24.74 & $22.50 \%$ & NO & YES & YES & $\mathrm{NO}$ \\
\hline 1290 & E9PBL7 & E9PBL7 & 79.68 & $12.40 \%$ & $\mathrm{NO}$ & $\mathrm{NO}$ & NO & NO \\
\hline 1291 & Q9UNE7 & CHIP & 34.86 & $12.50 \%$ & NO & YES & NO & NO \\
\hline 1292 & Q15642 & CIP4 & 67.73 & $8.82 \%$ & NO & NO & NO & NO \\
\hline 1293 & Q9NZL9 & MAT2B & 37.55 & $10.50 \%$ & YES & YES & NO & $\mathrm{NO}$ \\
\hline 1294 & Q9BSJ8 & ESYT1 & 122.86 & $3.99 \%$ & YES & NO & YES & NO \\
\hline 1295 & B3KQC5 & B3KQC5 & 79.58 & $11.20 \%$ & NO & YES & NO & NO \\
\hline 1296 & Q9UNS2 & CSN3 & 47.87 & $9.22 \%$ & NO & YES & YES & NO \\
\hline 1297 & O95502 & NPTXR & 52.85 & $12.00 \%$ & NO & NO & YES & $\mathrm{NO}$ \\
\hline 1298 & O15355 & PPM1G & 59.27 & $4.76 \%$ & $\mathrm{NO}$ & $\mathrm{NO}$ & NO & NO \\
\hline 1299 & A3KFL2 & A3KFL4 & 32.79 & $16.00 \%$ & NO & YES & NO & NO \\
\hline 1300 & F5GX82 & F5GX82 & 339.60 & $1.43 \%$ & NO & NO & NO & NO \\
\hline 1301 & O94855 & SC24D & 113.01 & $4.07 \%$ & NO & YES & NO & $\mathrm{NO}$ \\
\hline 1302 & O60488 & ACSL4 & 79.19 & $4.36 \%$ & $\mathrm{NO}$ & $\mathrm{NO}$ & NO & NO \\
\hline 1303 & Q15404 & RSU1 & 31.54 & $15.50 \%$ & YES & YES & YES & NO \\
\hline 1304 & Q9BZE9 & ASPC1 & 60.18 & $5.97 \%$ & NO & NO & NO & NO \\
\hline 1305 & Q9NT62 & ATG3 & 35.86 & $7.32 \%$ & NO & YES & NO & NO \\
\hline 1306 & Q9Y281 & COF2 & 18.74 & $39.80 \%$ & NO & YES & YES & NO \\
\hline 1307 & P51452 & DUS3 & 20.48 & $13.50 \%$ & NO & YES & NO & NO \\
\hline 1308 & H0Y5R9 & F8W031 & 29.22 & $15.60 \%$ & NO & YES & NO & $\mathrm{NO}$ \\
\hline 1309 & P35268 & RL22 & 14.79 & $28.90 \%$ & YES & YES & YES & NO \\
\hline 1310 & O43615 & TIM44 & 51.36 & $5.09 \%$ & NO & YES & NO & NO \\
\hline 1311 & Q8NBT2 & SPC24 & 22.48 & $21.80 \%$ & NO & YES & NO & $\mathrm{NO}$ \\
\hline 1312 & F5H2H7 & F5H2H7 & 83.12 & $3.48 \%$ & YES & NO & NO & $\mathrm{NO}$ \\
\hline 1313 & Q9NY97 & B3GN2 & 46.02 & $6.05 \%$ & YES & NO & YES & NO \\
\hline 1314 & P13598 & ICAM2 & 30.65 & $11.30 \%$ & NO & NO & YES & NO \\
\hline 1315 & E9PJT1 & E9PN05 & 70.76 & $5.81 \%$ & NO & YES & NO & NO \\
\hline 1316 & Q1ET66 & Q1ET66 & 34.20 & $7.64 \%$ & NO & YES & NO & $\mathrm{NO}$ \\
\hline 1317 & O15160 & RPAC1 & 39.25 & $9.25 \%$ & YES & $\mathrm{NO}$ & NO & NO \\
\hline 1318 & Q93052 & LPP & 65.75 & $6.05 \%$ & YES & YES & NO & NO \\
\hline 1319 & O43865 & SAHH2 & 58.95 & $6.04 \%$ & NO & NO & NO & $\mathrm{NO}$ \\
\hline 1320 & Q9H0E2 & TOLIP & 30.28 & $8.39 \%$ & NO & NO & YES & $\mathrm{NO}$ \\
\hline 1321 & Q27J81 & INF2 & 135.63 & $4.00 \%$ & $\mathrm{NO}$ & $\mathrm{NO}$ & NO & NO \\
\hline 1322 & P12955 & PEPD & 54.55 & $8.72 \%$ & NO & NO & YES & NO \\
\hline 1323 & P15374 & UCHL3 & 26.18 & $17.00 \%$ & NO & YES & NO & $\mathrm{NO}$ \\
\hline 1324 & Q92797 & SYMPK & 141.15 & $2.20 \%$ & NO & NO & NO & $\mathrm{NO}$ \\
\hline 1325 & P30519 & HMOX2 & 36.03 & $16.10 \%$ & NO & NO & YES & NO \\
\hline 1326 & Q8TBC4 & UBA3 & 51.85 & $10.20 \%$ & NO & YES & NO & $\mathrm{NO}$ \\
\hline 1327 & Q7Z4H8 & KDEL2 & 58.57 & $8.88 \%$ & NO & NO & NO & $\mathrm{NO}$ \\
\hline 1328 & P07099 & HYEP & 52.95 & $6.15 \%$ & NO & NO & NO & NO \\
\hline 1329 & Q9ULC4 & MCTS1 & 20.56 & $19.30 \%$ & NO & NO & NO & NO \\
\hline 1330 & Q5M775 & CYTSB & 118.59 & $2.53 \%$ & NO & NO & NO & NO \\
\hline 1331 & Q9H4G0 & E41L1 & 98.51 & $4.20 \%$ & YES & NO & NO & $\mathrm{NO}$ \\
\hline 1332 & P30533 & AMRP & 41.47 & $5.60 \%$ & NO & NO & NO & $\mathrm{NO}$ \\
\hline 1333 & F5H4E0 & F2Z2B9 & 102.54 & $3.33 \%$ & NO & NO & NO & NO \\
\hline 1334 & P78318 & IGBP1 & 39.22 & $6.49 \%$ & YES & NO & NO & NO \\
\hline 1335 & Q7Z434 & MAVS & 56.53 & $6.11 \%$ & NO & NO & NO & NO \\
\hline 1336 & O00442 & RTCA & 39.34 & $6.56 \%$ & $\mathrm{NO}$ & YES & NO & NO \\
\hline 1337 & P22059 & OSBP1 & 89.42 & $2.48 \%$ & NO & NO & NO & NO \\
\hline
\end{tabular}




\begin{tabular}{|c|c|c|c|c|c|c|c|c|c|}
\hline 1338 & P05109 & S10A8 & 10.84 & $20.40 \%$ & NO & NO & YES & NO & $\mathrm{NO}$ \\
\hline 1339 & A1L4H1 & SRCRL & 165.74 & $1.59 \%$ & $\mathrm{NO}$ & NO & YES & $\mathrm{NO}$ & $\mathrm{NO}$ \\
\hline 1340 & Q9BWJ5 & SF3B5 & 10.14 & $25.60 \%$ & YES & YES & $\mathrm{NO}$ & NO & $\mathrm{NO}$ \\
\hline 1341 & F5H012 & F5H012 & 54.17 & $3.79 \%$ & NO & NO & NO & NO & $\mathrm{NO}$ \\
\hline 1342 & O00203 & AP3B1 & 121.32 & $1.74 \%$ & YES & NO & $\mathrm{NO}$ & NO & $\mathrm{NO}$ \\
\hline 1343 & C9JLZ0 & C9JLZ0 & 125.87 & $1.94 \%$ & NO & $\mathrm{NO}$ & $\mathrm{NO}$ & $\mathrm{NO}$ & $\mathrm{NO}$ \\
\hline 1344 & Q9BR76 & COR1B & 54.23 & $4.29 \%$ & NO & YES & YES & NO & NO \\
\hline 1345 & E5RH77 & E5RH77 & 14.30 & $23.10 \%$ & NO & YES & NO & NO & $\mathrm{NO}$ \\
\hline 1346 & H7C3A1 & H7C3A1 & 100.67 & $3.61 \%$ & NO & NO & $\mathrm{NO}$ & $\mathrm{NO}$ & $\mathrm{NO}$ \\
\hline 1347 & Q9Y3C0 & CCD53 & 21.17 & $13.40 \%$ & NO & YES & $\mathrm{NO}$ & $\mathrm{NO}$ & $\mathrm{NO}$ \\
\hline 1348 & Q6P1N9 & TATD1 & 33.60 & $7.07 \%$ & NO & NO & $\mathrm{NO}$ & NO & NO \\
\hline 1349 & O75144 & ICOSL & 33.35 & $6.62 \%$ & NO & NO & YES & NO & $\mathrm{NO}$ \\
\hline 1350 & Q9P2R3 & ANFY1 & 128.40 & $3.93 \%$ & NO & NO & YES & $\mathrm{NO}$ & $\mathrm{NO}$ \\
\hline 1351 & O00300 & TR11B & 46.03 & $9.73 \%$ & NO & NO & YES & $\mathrm{NO}$ & $\mathrm{NO}$ \\
\hline 1352 & P55265 & DSRAD & 136.07 & $4.24 \%$ & NO & NO & NO & NO & $\mathrm{NO}$ \\
\hline 1353 & J3QLD9 & E7EMK3 & 47.06 & $7.66 \%$ & NO & NO & NO & NO & $\mathrm{NO}$ \\
\hline 1354 & P50583 & AP4A & 16.83 & $21.80 \%$ & NO & YES & $\mathrm{NO}$ & $\mathrm{NO}$ & $\mathrm{NO}$ \\
\hline 1355 & Q8N8Z6 & DCBD1 & 77.92 & $6.99 \%$ & NO & $\mathrm{NO}$ & $\mathrm{NO}$ & $\mathrm{NO}$ & $\mathrm{NO}$ \\
\hline 1356 & E9PGT0 & E9PGT0 & 358.20 & $0.98 \%$ & NO & NO & NO & NO & $\mathrm{NO}$ \\
\hline 1357 & A8MX49 & A8MX49 & 49.83 & $8.59 \%$ & YES & YES & NO & NO & $\mathrm{NO}$ \\
\hline 1358 & Q13177 & PAK2 & 58.04 & $4.96 \%$ & YES & NO & $\mathrm{NO}$ & $\mathrm{NO}$ & $\mathrm{NO}$ \\
\hline 1359 & Q9HDC9 & APMAP & 46.48 & $8.41 \%$ & NO & YES & $\mathrm{NO}$ & $\mathrm{NO}$ & $\mathrm{NO}$ \\
\hline 1360 & E9PJ81 & E9PJ81 & 33.33 & $8.97 \%$ & NO & YES & NO & NO & $\mathrm{NO}$ \\
\hline 1361 & Q7Z2Z2 & ETUD1 & 125.43 & $2.86 \%$ & NO & NO & $\mathrm{NO}$ & NO & $\mathrm{NO}$ \\
\hline 1362 & Q6YP21 & KAT3 & 51.40 & $5.51 \%$ & NO & YES & $\mathrm{NO}$ & $\mathrm{NO}$ & $\mathrm{NO}$ \\
\hline 1363 & F5H296 & F5H296 & 9.20 & $31.80 \%$ & NO & YES & $\mathrm{NO}$ & $\mathrm{NO}$ & $\mathrm{NO}$ \\
\hline 1364 & O95833 & CLIC3 & 26.65 & $10.20 \%$ & NO & YES & YES & NO & $\mathrm{NO}$ \\
\hline 1365 & E9PCB6 & E9PCB6 & 80.65 & $5.67 \%$ & YES & NO & $\mathrm{NO}$ & NO & NO \\
\hline 1366 & O14907 & TX1B3 & 13.73 & $28.20 \%$ & NO & YES & $\mathrm{NO}$ & $\mathrm{NO}$ & $\mathrm{NO}$ \\
\hline 1367 & P62487 & RPB7 & 19.29 & $14.00 \%$ & YES & NO & $\mathrm{NO}$ & NO & $\mathrm{NO}$ \\
\hline 1368 & Р23634 & AT2B4 & 137.92 & $2.74 \%$ & NO & NO & NO & NO & NO \\
\hline 1369 & Q96G03 & PGM2 & 68.29 & $4.41 \%$ & NO & $\mathrm{NO}$ & YES & $\mathrm{NO}$ & $\mathrm{NO}$ \\
\hline 1370 & Н3ВР04 & Н3ВР04 & 48.44 & $22.20 \%$ & NO & YES & NO & $\mathrm{NO}$ & $\mathrm{NO}$ \\
\hline 1371 & P22455 & FGFR4 & 87.96 & $3.12 \%$ & NO & NO & $\mathrm{NO}$ & NO & $\mathrm{NO}$ \\
\hline 1372 & A6NEM2 & A6NEM2 & 208.72 & $1.06 \%$ & NO & NO & NO & NO & NO \\
\hline 1373 & Q96QR8 & PURB & 33.24 & $12.20 \%$ & YES & $\mathrm{NO}$ & NO & $\mathrm{NO}$ & $\mathrm{NO}$ \\
\hline 1374 & B4DUT8 & B4DDF4 & 33.70 & $9.40 \%$ & YES & NO & NO & $\mathrm{NO}$ & $\mathrm{NO}$ \\
\hline 1375 & B1AKR6 & B1AKR6 & 16.25 & $25.00 \%$ & NO & NO & $\mathrm{NO}$ & NO & $\mathrm{NO}$ \\
\hline 1376 & A6NG10 & A6NG10 & 28.09 & $6.69 \%$ & NO & NO & NO & NO & NO \\
\hline 1377 & O43488 & ARK72 & 39.59 & $10.90 \%$ & NO & YES & YES & $\mathrm{NO}$ & $\mathrm{NO}$ \\
\hline 1378 & O94874 & UFL1 & 89.60 & $5.29 \%$ & NO & NO & NO & $\mathrm{NO}$ & $\mathrm{NO}$ \\
\hline 1379 & O75063 & XYLK & 46.43 & $7.82 \%$ & NO & $\mathrm{NO}$ & $\mathrm{NO}$ & $\mathrm{NO}$ & $\mathrm{NO}$ \\
\hline 1380 & P01111 & RASN & 21.23 & $18.50 \%$ & NO & YES & YES & NO & $\mathrm{NO}$ \\
\hline 1381 & Q7LGC8 & CHST3 & 54.71 & $5.64 \%$ & NO & YES & $\mathrm{NO}$ & $\mathrm{NO}$ & $\mathrm{NO}$ \\
\hline 1382 & Q14108 & E7EM68 & 54.29 & $6.49 \%$ & YES & NO & YES & NO & $\mathrm{NO}$ \\
\hline 1383 & F5H4Z4 & F5H4Z4 & 88.34 & $4.00 \%$ & $\mathrm{NO}$ & YES & $\mathrm{NO}$ & $\mathrm{NO}$ & $\mathrm{NO}$ \\
\hline 1384 & O75934 & SPF27 & 26.13 & $12.00 \%$ & NO & YES & NO & NO & $\mathrm{NO}$ \\
\hline 1385 & Q9NR09 & BIRC6 & 530.25 & $0.68 \%$ & YES & $\mathrm{NO}$ & $\mathrm{NO}$ & $\mathrm{NO}$ & $\mathrm{NO}$ \\
\hline 1386 & F5H2U2 & F5H2U2 & 116.99 & $2.42 \%$ & YES & NO & $\mathrm{NO}$ & NO & $\mathrm{NO}$ \\
\hline 1387 & P55795 & HNRH2 & 49.26 & $17.40 \%$ & NO & NO & NO & NO & NO \\
\hline 1388 & P46939 & UTRO & 394.47 & $1.05 \%$ & NO & NO & NO & NO & $\mathrm{NO}$ \\
\hline 1389 & Q9GZL7 & WDR12 & 47.71 & $5.20 \%$ & NO & YES & NO & NO & NO \\
\hline 1390 & B4E322 & B4E322 & 41.73 & $9.45 \%$ & YES & YES & $\mathrm{NO}$ & $\mathrm{NO}$ & $\mathrm{NO}$ \\
\hline 1391 & E9PB21 & E9PB21 & 24.54 & $6.67 \%$ & NO & YES & $\mathrm{NO}$ & NO & $\mathrm{NO}$ \\
\hline 1392 & P31930 & QCR1 & 52.65 & $6.46 \%$ & NO & YES & YES & NO & $\mathrm{NO}$ \\
\hline 1393 & B5MDN3 & B5MDN3 & 266.94 & $1.47 \%$ & NO & NO & $\mathrm{NO}$ & NO & $\mathrm{NO}$ \\
\hline
\end{tabular}




\begin{tabular}{|c|c|c|c|c|c|c|c|c|}
\hline 1394 & Q96HD1 & CREL1 & 45.44 & $5.48 \%$ & NO & NO & YES & NO \\
\hline 1395 & O75506 & HSBP1 & 8.54 & $39.50 \%$ & NO & NO & $\mathrm{NO}$ & $\mathrm{NO}$ \\
\hline 1396 & B1AH49 & B1AH49 & 33.18 & $13.80 \%$ & NO & NO & $\mathrm{NO}$ & $\mathrm{NO}$ \\
\hline 1397 & B4DJS7 & B4DJS7 & 46.65 & $6.80 \%$ & YES & NO & NO & $\mathrm{NO}$ \\
\hline 1398 & $\mathrm{~A} 2 \mathrm{~A} 2 \mathrm{Q} 9$ & $\mathrm{~A} 2 \mathrm{~A} 2 \mathrm{Q} 9$ & 43.47 & $7.54 \%$ & NO & NO & NO & NO \\
\hline 1399 & Q9UK59 & DBR1 & 61.56 & $5.88 \%$ & NO & NO & NO & $\mathrm{NO}$ \\
\hline 1400 & Q9H993 & CF211 & 51.17 & $3.85 \%$ & NO & YES & NO & NO \\
\hline 1401 & B4DH53 & B4DH53 & 112.21 & $2.42 \%$ & NO & NO & NO & NO \\
\hline 1402 & P80217 & IN35 & 31.55 & $10.80 \%$ & $\mathrm{NO}$ & $\mathrm{NO}$ & NO & $\mathrm{NO}$ \\
\hline 1403 & O96019 & ACL6A & 47.46 & $5.59 \%$ & NO & NO & $\mathrm{NO}$ & $\mathrm{NO}$ \\
\hline 1404 & C9JEV0 & C9JEV0 & 34.26 & $14.50 \%$ & NO & NO & NO & NO \\
\hline 1405 & Q8TEA8 & DTD1 & 23.42 & $17.70 \%$ & NO & YES & NO & $\mathrm{NO}$ \\
\hline 1406 & Q9H3P7 & GCP60 & 60.59 & $3.03 \%$ & NO & YES & NO & NO \\
\hline 1407 & C9JDM9 & C9JDM9 & 329.48 & $2.72 \%$ & NO & NO & NO & NO \\
\hline 1408 & Е9PРJ0 & Е9PРJ0 & 100.23 & $2.16 \%$ & NO & NO & NO & $\mathrm{NO}$ \\
\hline 1409 & Q86X55 & CARM1 & 65.85 & $2.80 \%$ & NO & YES & NO & NO \\
\hline 1410 & Q9BVJ7 & DUS23 & 16.59 & $14.70 \%$ & NO & YES & NO & NO \\
\hline 1411 & P57678 & GEMI4 & 120.04 & $3.31 \%$ & NO & YES & $\mathrm{NO}$ & $\mathrm{NO}$ \\
\hline 1412 & Q13643 & FHL3 & 31.19 & $9.64 \%$ & YES & YES & NO & NO \\
\hline 1413 & E5RG77 & H0YBB9 & 30.34 & $10.70 \%$ & NO & NO & NO & NO \\
\hline 1414 & H0Y855 & C9JXC1 & 51.09 & $10.00 \%$ & NO & NO & NO & NO \\
\hline 1415 & F5GXG0 & F5GXG0 & 89.81 & $2.40 \%$ & NO & NO & NO & NO \\
\hline 1416 & P40261 & NNMT & 29.58 & $11.40 \%$ & NO & NO & NO & NO \\
\hline 1417 & Q9UKN8 & TF3C4 & 91.98 & $3.77 \%$ & NO & YES & NO & NO \\
\hline 1418 & Q8IXK2 & GLT12 & 66.94 & $4.99 \%$ & YES & NO & NO & NO \\
\hline 1419 & Q96L58 & B3GT6 & 37.14 & $6.08 \%$ & NO & NO & $\mathrm{NO}$ & $\mathrm{NO}$ \\
\hline 1420 & Q7KZ85 & SPT6H & 199.08 & $1.45 \%$ & YES & NO & NO & NO \\
\hline 1421 & Q16763 & UBE2S & 23.85 & $5.86 \%$ & YES & NO & NO & NO \\
\hline 1422 & P23458 & JAK1 & 133.28 & $2.17 \%$ & NO & NO & NO & NO \\
\hline 1423 & B0AZT5 & E7EQ69 & 19.40 & $14.30 \%$ & NO & YES & NO & NO \\
\hline 1424 & B7Z3I9 & B7Z3I9 & 36.30 & $7.67 \%$ & NO & YES & $\mathrm{NO}$ & $\mathrm{NO}$ \\
\hline 1425 & Q9BRF8 & CPPED & 35.55 & $13.10 \%$ & YES & NO & YES & NO \\
\hline 1426 & P35475 & IDUA & 72.67 & $3.68 \%$ & NO & NO & $\mathrm{NO}$ & $\mathrm{NO}$ \\
\hline 1427 & F2Z2E2 & F2Z2E2 & 184.70 & $2.08 \%$ & NO & NO & NO & NO \\
\hline 1428 & B5MCL2 & B5MCL2 & 27.51 & $11.70 \%$ & NO & YES & NO & $\mathrm{NO}$ \\
\hline 1429 & O95571 & ETHE1 & 27.87 & $10.60 \%$ & NO & YES & NO & NO \\
\hline 1430 & P10599 & THIO & 11.74 & $63.80 \%$ & NO & NO & YES & NO \\
\hline 1431 & P23284 & PPIB & 23.74 & $38.40 \%$ & YES & NO & YES & NO \\
\hline 1432 & G5EA09 & G5EA09 & 32.45 & $45.60 \%$ & NO & NO & NO & NO \\
\hline 1433 & С9J9K3 & С9J9K3 & 32.85 & $47.30 \%$ & NO & NO & NO & NO \\
\hline 1434 & P26599 & PTBP1 & 57.22 & $35.20 \%$ & NO & NO & YES & $\mathrm{NO}$ \\
\hline 1435 & O75874 & IDHC & 46.66 & $24.90 \%$ & NO & YES & YES & NO \\
\hline 1436 & O14818 & PSA7 & 27.89 & $50.40 \%$ & NO & NO & YES & NO \\
\hline 1437 & Q9Y617 & SERC & 40.42 & $35.40 \%$ & NO & NO & YES & NO \\
\hline 1438 & Q14195 & DPYL3 & 61.96 & $34.40 \%$ & NO & NO & NO & NO \\
\hline 1439 & P17174 & AATC & 46.25 & $32.20 \%$ & NO & NO & YES & NO \\
\hline 1440 & O43390 & HNRPR & 70.94 & $24.60 \%$ & NO & NO & NO & NO \\
\hline 1441 & Q16706 & MA2A1 & 131.15 & $12.20 \%$ & NO & NO & YES & NO \\
\hline 1442 & Q9NYU2 & UGGG1 & 177.20 & $9.32 \%$ & NO & NO & $\mathrm{NO}$ & $\mathrm{NO}$ \\
\hline 1443 & P61289 & PSME3 & 29.51 & $28.30 \%$ & NO & NO & NO & NO \\
\hline 1444 & Q9NQG5 & RPR1B & 36.90 & $15.30 \%$ & YES & NO & NO & NO \\
\hline 1445 & P51858 & HDGF & 26.79 & $14.60 \%$ & NO & NO & NO & NO \\
\hline 1446 & B1AK88 & B1AK87 & 33.78 & $20.40 \%$ & NO & YES & NO & NO \\
\hline 1447 & А8MXР9 & А 8 MXР9 & 94.63 & $10.80 \%$ & NO & NO & NO & NO \\
\hline 1448 & Q8NBJ5 & GT251 & 71.64 & $9.00 \%$ & $\mathrm{NO}$ & NO & NO & NO \\
\hline 1449 & C9JEJ7 & C9JBI3 & 25.01 & $29.40 \%$ & NO & YES & $\mathrm{NO}$ & $\mathrm{NO}$ \\
\hline
\end{tabular}




\begin{tabular}{|c|c|c|c|c|c|c|c|c|c|}
\hline 1450 & P60903 & S10AA & 11.20 & $35.10 \%$ & $\mathrm{NO}$ & NO & YES & NO & $\mathrm{NO}$ \\
\hline 1451 & Q5JPE7 & NOMO2 & 139.44 & $7.10 \%$ & $\mathrm{NO}$ & NO & $\mathrm{NO}$ & $\mathrm{NO}$ & $\mathrm{NO}$ \\
\hline 1452 & Q86SR1 & GLT10 & 68.99 & $10.90 \%$ & NO & $\mathrm{NO}$ & $\mathrm{NO}$ & NO & $\mathrm{NO}$ \\
\hline 1453 & Q9BQT9 & CSTN3 & 106.10 & $8.79 \%$ & YES & NO & YES & NO & $\mathrm{NO}$ \\
\hline 1454 & P02792 & FRIL & 20.02 & $21.10 \%$ & NO & NO & YES & NO & $\mathrm{NO}$ \\
\hline 1455 & P68402 & PA1B2 & 25.57 & $15.30 \%$ & NO & NO & YES & NO & $\mathrm{NO}$ \\
\hline 1456 & O94760 & DDAH1 & 31.12 & $22.10 \%$ & NO & NO & YES & NO & $\mathrm{NO}$ \\
\hline 1457 & Q9NUQ9 & FA49B & 36.75 & $18.20 \%$ & NO & NO & YES & NO & $\mathrm{NO}$ \\
\hline 1458 & P09104 & ENOG & 47.27 & $34.60 \%$ & NO & YES & $\mathrm{NO}$ & NO & $\mathrm{NO}$ \\
\hline 1459 & Q86XP3 & DDX42 & 102.98 & $8.53 \%$ & YES & $\mathrm{NO}$ & $\mathrm{NO}$ & $\mathrm{NO}$ & NO \\
\hline 1460 & A8KAH5 & A8KAH5 & 39.75 & $8.97 \%$ & NO & NO & $\mathrm{NO}$ & NO & NO \\
\hline 1461 & O43242 & PSMD3 & 60.98 & $8.05 \%$ & NO & NO & YES & NO & $\mathrm{NO}$ \\
\hline 1462 & Q53FA7 & QORX & 35.54 & $14.80 \%$ & NO & YES & YES & NO & $\mathrm{NO}$ \\
\hline 1463 & B5MBZ0 & B5MBZ0 & 108.92 & $4.13 \%$ & NO & NO & NO & NO & $\mathrm{NO}$ \\
\hline 1464 & Q9BVG4 & CX026 & 26.06 & $15.50 \%$ & NO & NO & NO & NO & NO \\
\hline 1465 & Q01433 & AMPD2 & 100.69 & $2.39 \%$ & NO & NO & NO & NO & NO \\
\hline 1466 & E7ES17 & E7ES17 & 116.22 & $3.35 \%$ & NO & NO & NO & NO & $\mathrm{NO}$ \\
\hline 1467 & A2A2N2 & $\mathrm{A} 2 \mathrm{~A} 2 \mathrm{~N} 2$ & 21.45 & $19.60 \%$ & YES & YES & $\mathrm{NO}$ & $\mathrm{NO}$ & $\mathrm{NO}$ \\
\hline 1468 & E9PIE3 & E9PIE3 & 27.70 & $7.17 \%$ & NO & NO & NO & NO & $\mathrm{NO}$ \\
\hline 1469 & B4DP31 & B4DP31 & 39.39 & $9.57 \%$ & YES & NO & NO & NO & $\mathrm{NO}$ \\
\hline 1470 & Q08752 & PPID & 40.76 & $4.86 \%$ & NO & NO & NO & NO & $\mathrm{NO}$ \\
\hline 1471 & F8WE33 & F5H7K4 & 45.81 & $6.25 \%$ & NO & YES & NO & NO & $\mathrm{NO}$ \\
\hline 1472 & Q12996 & CSTF3 & 82.92 & $4.32 \%$ & NO & NO & NO & NO & $\mathrm{NO}$ \\
\hline 1473 & E7ENA9 & E7ENA9 & 78.41 & $6.65 \%$ & NO & NO & NO & NO & $\mathrm{NO}$ \\
\hline 1474 & Q96ST3 & SIN3A & 145.18 & $2.04 \%$ & NO & NO & NO & NO & $\mathrm{NO}$ \\
\hline 1475 & P63172 & DYLT1 & 12.45 & $24.80 \%$ & NO & YES & $\mathrm{NO}$ & $\mathrm{NO}$ & $\mathrm{NO}$ \\
\hline 1476 & O14656 & TOR1A & 37.81 & $6.93 \%$ & NO & NO & NO & NO & $\mathrm{NO}$ \\
\hline 1477 & Q9HCU4 & CELR2 & 317.45 & $1.33 \%$ & NO & NO & NO & NO & $\mathrm{NO}$ \\
\hline 1478 & Q15223 & PVRL1 & 57.16 & $9.48 \%$ & NO & NO & YES & NO & $\mathrm{NO}$ \\
\hline 1479 & $\mathrm{~J} 3 \mathrm{KS} 23$ & J3KS23 & 21.51 & $31.70 \%$ & YES & YES & $\mathrm{NO}$ & NO & NO \\
\hline 1480 & D6R9S0 & D6RBJ9 & 20.02 & $11.20 \%$ & NO & YES & NO & NO & $\mathrm{NO}$ \\
\hline 1481 & Q96MX6 & WDR92 & 39.74 & $3.64 \%$ & NO & NO & $\mathrm{NO}$ & NO & $\mathrm{NO}$ \\
\hline 1482 & D6RH03 & D6RH03 & 103.54 & $9.61 \%$ & NO & NO & NO & $\mathrm{NO}$ & $\mathrm{NO}$ \\
\hline 1483 & F5H4Z3 & F5H4Z3 & 29.82 & $11.40 \%$ & NO & YES & $\mathrm{NO}$ & NO & $\mathrm{NO}$ \\
\hline 1484 & O60512 & B4GT3 & 43.93 & $3.82 \%$ & NO & NO & NO & NO & $\mathrm{NO}$ \\
\hline 1485 & Q08379 & GOGA2 & 113.09 & $2.50 \%$ & NO & NO & $\mathrm{NO}$ & NO & $\mathrm{NO}$ \\
\hline 1486 & B4DEH8 & B4DEH8 & 32.75 & $23.20 \%$ & NO & YES & NO & NO & $\mathrm{NO}$ \\
\hline 1487 & J3KNK7 & E7EMH5 & 86.71 & $5.60 \%$ & NO & NO & $\mathrm{NO}$ & NO & $\mathrm{NO}$ \\
\hline 1488 & Q13253 & NOGG & 25.77 & $23.30 \%$ & NO & NO & NO & NO & $\mathrm{NO}$ \\
\hline 1489 & P35251 & RFC1 & 128.26 & $2.00 \%$ & NO & NO & $\mathrm{NO}$ & NO & $\mathrm{NO}$ \\
\hline 1490 & Q8IXB1 & DJC10 & 91.08 & $2.77 \%$ & YES & YES & $\mathrm{NO}$ & NO & $\mathrm{NO}$ \\
\hline 1491 & Q8IW52 & SLIK4 & 94.34 & $2.63 \%$ & YES & NO & $\mathrm{NO}$ & NO & $\mathrm{NO}$ \\
\hline 1492 & Q6UWE0 & LRSM1 & 83.60 & $4.29 \%$ & YES & NO & NO & NO & $\mathrm{NO}$ \\
\hline 1493 & Q9NRN7 & ADPPT & 35.78 & $6.80 \%$ & NO & YES & NO & NO & $\mathrm{NO}$ \\
\hline 1494 & P60709 & АСТВ & 41.74 & $64.80 \%$ & NO & NO & YES & NO & $\mathrm{NO}$ \\
\hline 1495 & Q15149 & PLEC & 531.78 & $24.70 \%$ & NO & NO & YES & NO & $\mathrm{NO}$ \\
\hline 1496 & P10809 & CH60 & 61.06 & $45.90 \%$ & YES & NO & YES & NO & $\mathrm{NO}$ \\
\hline 1497 & Q92598 & HS105 & 96.87 & $29.60 \%$ & YES & $\mathrm{NO}$ & $\mathrm{NO}$ & NO & $\mathrm{NO}$ \\
\hline 1498 & Q01518 & CAP1 & 51.90 & $32.80 \%$ & YES & NO & YES & NO & $\mathrm{NO}$ \\
\hline 1499 & P13645 & K1C10 & 58.83 & $39.90 \%$ & NO & NO & YES & NO & $\mathrm{NO}$ \\
\hline 1500 & P61981 & $1433 \mathrm{G}$ & 28.30 & $38.10 \%$ & YES & NO & YES & NO & $\mathrm{NO}$ \\
\hline 1501 & J9JID7 & J9JID7 & 67.69 & $12.30 \%$ & NO & NO & NO & NO & $\mathrm{NO}$ \\
\hline 1502 & J3KQ69 & J3KQ69 & 90.98 & $14.30 \%$ & $\mathrm{NO}$ & NO & $\mathrm{NO}$ & $\mathrm{NO}$ & $\mathrm{NO}$ \\
\hline 1503 & B4DQV7 & B4DQV7 & 16.57 & $52.00 \%$ & NO & NO & $\mathrm{NO}$ & NO & $\mathrm{NO}$ \\
\hline 1504 & E7EWS7 & E7EWS7 & 17.86 & $26.40 \%$ & NO & NO & $\mathrm{NO}$ & NO & $\mathrm{NO}$ \\
\hline 1505 & O75882 & ATRN & 158.54 & $5.04 \%$ & NO & NO & YES & $\mathrm{NO}$ & $\mathrm{NO}$ \\
\hline
\end{tabular}




\begin{tabular}{|c|c|c|c|c|c|c|c|c|}
\hline 1506 & Q9Y383 & LC7L2 & 46.51 & $8.93 \%$ & YES & YES & NO & $\mathrm{NO}$ \\
\hline 1507 & Q15691 & MARE1 & 30.00 & $17.50 \%$ & NO & NO & NO & NO \\
\hline 1508 & C9K025 & С9K025 & 12.54 & $17.00 \%$ & NO & YES & $\mathrm{NO}$ & NO \\
\hline 1509 & Q9H1B5 & XYLT2 & 96.77 & $5.66 \%$ & NO & NO & YES & NO \\
\hline 1510 & Q07666 & KHDR1 & 48.23 & $9.93 \%$ & NO & $\mathrm{NO}$ & $\mathrm{NO}$ & NO \\
\hline 1511 & B4E1Z4 & B4E1Z4 & 85.53 & $6.64 \%$ & NO & $\mathrm{NO}$ & $\mathrm{NO}$ & NO \\
\hline 1512 & P98172 & EFNB1 & 38.01 & $9.83 \%$ & NO & NO & YES & NO \\
\hline 1513 & Q5T8U5 & Q5T8U5 & 30.40 & $21.00 \%$ & NO & YES & $\mathrm{NO}$ & $\mathrm{NO}$ \\
\hline 1514 & O94766 & B3GA3 & 37.12 & $9.25 \%$ & $\mathrm{NO}$ & $\mathrm{NO}$ & NO & NO \\
\hline 1515 & P49207 & RL34 & 13.29 & $12.80 \%$ & NO & YES & $\mathrm{NO}$ & NO \\
\hline 1516 & P40939 & ECHA & 83.00 & $5.64 \%$ & YES & NO & YES & NO \\
\hline 1517 & B3KV55 & B4DGN5 & 61.40 & $8.94 \%$ & NO & NO & $\mathrm{NO}$ & $\mathrm{NO}$ \\
\hline 1518 & Q86SJ2 & AMGO2 & 57.94 & $6.70 \%$ & NO & NO & NO & NO \\
\hline 1519 & Q9H444 & CHM4B & 24.95 & $10.30 \%$ & NO & NO & YES & NO \\
\hline 1520 & Q11201 & SIA4A & 39.08 & $10.30 \%$ & YES & NO & NO & NO \\
\hline 1521 & B4DEJ6 & В3КТ28 & 53.65 & $4.74 \%$ & NO & YES & NO & $\mathrm{NO}$ \\
\hline 1522 & P62993 & GRB2 & 25.21 & $18.00 \%$ & $\mathrm{NO}$ & $\mathrm{NO}$ & YES & NO \\
\hline 1523 & Q10469 & MGAT2 & 51.55 & $6.94 \%$ & NO & NO & $\mathrm{NO}$ & NO \\
\hline 1524 & F5H442 & F5H442 & 43.95 & $6.58 \%$ & NO & YES & NO & NO \\
\hline 1525 & P24752 & THIL & 45.20 & $6.09 \%$ & NO & YES & YES & $\mathrm{NO}$ \\
\hline 1526 & H7C449 & H7BZM7 & 50.93 & $3.95 \%$ & $\mathrm{NO}$ & YES & $\mathrm{NO}$ & NO \\
\hline 1527 & C9JWC5 & C9JWC5 & 56.95 & $12.50 \%$ & YES & YES & NO & $\mathrm{NO}$ \\
\hline 1528 & F5GY99 & F5GY99 & 64.22 & $1.80 \%$ & YES & NO & NO & NO \\
\hline 1529 & Q96CV9 & OPTN & 65.92 & $3.99 \%$ & NO & NO & $\mathrm{NO}$ & NO \\
\hline 1530 & O75976 & CBPD & 152.93 & $1.67 \%$ & NO & NO & NO & NO \\
\hline 1531 & A8MUS3 & A8MUS3 & 17.80 & $11.90 \%$ & NO & YES & $\mathrm{NO}$ & NO \\
\hline 1532 & Q5EBL8 & PDZ11 & 16.13 & $14.30 \%$ & NO & NO & $\mathrm{NO}$ & $\mathrm{NO}$ \\
\hline 1533 & Q3KQV9 & UAP1L & 57.03 & $7.10 \%$ & YES & NO & NO & NO \\
\hline 1534 & P08236 & BGLR & 74.73 & $2.76 \%$ & NO & NO & YES & NO \\
\hline 1535 & P11586 & $\mathrm{C} 1 \mathrm{TC}$ & 101.56 & $11.20 \%$ & NO & NO & YES & $\mathrm{NO}$ \\
\hline 1536 & P26373 & RL13 & 24.26 & $17.50 \%$ & YES & NO & YES & $\mathrm{NO}$ \\
\hline 1537 & B7Z5W8 & B7Z5W8 & 48.76 & $3.81 \%$ & $\mathrm{NO}$ & $\mathrm{NO}$ & $\mathrm{NO}$ & NO \\
\hline 1538 & Q9H2G2 & SLK & 142.70 & $2.83 \%$ & NO & NO & YES & NO \\
\hline 1539 & E7EWE1 & E7EQ61 & 44.86 & $13.80 \%$ & NO & YES & NO & NO \\
\hline 1540 & B4E2N0 & B4E2N0 & 23.93 & $15.60 \%$ & NO & YES & $\mathrm{NO}$ & $\mathrm{NO}$ \\
\hline 1541 & P35579 & MYH9 & 226.54 & $35.80 \%$ & $\mathrm{NO}$ & $\mathrm{NO}$ & YES & NO \\
\hline 1542 & P23528 & COF1 & 18.50 & $61.40 \%$ & NO & YES & $\mathrm{NO}$ & NO \\
\hline 1543 & P78371 & ТСРВ & 57.49 & $60.60 \%$ & NO & NO & YES & $\mathrm{NO}$ \\
\hline 1544 & Q14315 & FLNC & 291.02 & $27.80 \%$ & NO & NO & YES & $\mathrm{NO}$ \\
\hline 1545 & Q9Y4K0 & LOXL2 & 86.72 & $33.60 \%$ & $\mathrm{NO}$ & $\mathrm{NO}$ & $\mathrm{NO}$ & NO \\
\hline 1546 & P27348 & $1433 \mathrm{~T}$ & 27.77 & $55.90 \%$ & NO & NO & YES & NO \\
\hline 1547 & P46940 & IQGA1 & 189.26 & $33.00 \%$ & YES & NO & YES & $\mathrm{NO}$ \\
\hline 1548 & Q06830 & PRDX1 & 22.11 & $70.90 \%$ & NO & YES & YES & $\mathrm{NO}$ \\
\hline 1549 & Q14204 & DYHC1 & 532.41 & $13.10 \%$ & NO & NO & YES & NO \\
\hline 1550 & Q99832 & ТCPH & 59.37 & $42.70 \%$ & NO & NO & YES & $\mathrm{NO}$ \\
\hline 1551 & P11216 & PYGB & 96.70 & $28.10 \%$ & NO & NO & YES & $\mathrm{NO}$ \\
\hline 1552 & P63244 & GBLP & 35.08 & $53.60 \%$ & YES & NO & YES & NO \\
\hline 1553 & P52209 & 6PGD & 53.14 & $41.40 \%$ & YES & NO & YES & NO \\
\hline 1554 & P37802 & TAGL2 & 22.39 & $61.30 \%$ & NO & YES & YES & NO \\
\hline 1555 & Q13838 & DX39B & 48.99 & $39.50 \%$ & NO & YES & NO & $\mathrm{NO}$ \\
\hline 1556 & Q15233 & NONO & 54.23 & $28.70 \%$ & NO & NO & NO & NO \\
\hline 1557 & Q15029 & U5S1 & 109.44 & $23.40 \%$ & NO & NO & NO & $\mathrm{NO}$ \\
\hline 1558 & P25786 & PSA1 & 29.56 & $36.90 \%$ & NO & NO & YES & NO \\
\hline 1559 & O75643 & U520 & 244.51 & $9.60 \%$ & NO & NO & NO & NO \\
\hline 1560 & P62136 & PP1A & 37.51 & $40.00 \%$ & $\mathrm{NO}$ & YES & $\mathrm{NO}$ & NO \\
\hline 1561 & P84077 & ARF1 & 20.70 & $50.30 \%$ & NO & YES & YES & NO \\
\hline
\end{tabular}




\begin{tabular}{|c|c|c|c|c|c|c|c|c|}
\hline 1562 & P62829 & RL23 & 14.87 & $44.30 \%$ & NO & YES & NO & NO \\
\hline 1563 & P62906 & RL10A & 24.83 & $26.70 \%$ & NO & YES & NO & $\mathrm{NO}$ \\
\hline 1564 & Q92973 & TNPO1 & 102.36 & $15.40 \%$ & NO & $\mathrm{NO}$ & YES & NO \\
\hline 1565 & E5RI99 & E5RI99 & 12.78 & $46.50 \%$ & NO & NO & NO & NO \\
\hline 1566 & P04899 & GNAI2 & 40.45 & $26.50 \%$ & YES & YES & NO & $\mathrm{NO}$ \\
\hline 1567 & Q13561 & DCTN2 & 44.23 & $21.90 \%$ & $\mathrm{NO}$ & $\mathrm{NO}$ & NO & NO \\
\hline 1568 & F8WC70 & E7EUC7 & 56.94 & $13.70 \%$ & NO & YES & NO & NO \\
\hline 1569 & P30043 & BLVRB & 22.12 & $34.50 \%$ & NO & YES & YES & NO \\
\hline 1570 & E9PFM1 & D3DNT2 & 175.49 & $6.88 \%$ & NO & NO & NO & NO \\
\hline 1571 & O60513 & B4GT4 & 40.04 & $13.10 \%$ & YES & NO & NO & NO \\
\hline 1572 & P09972 & ALDOC & 39.46 & $23.10 \%$ & NO & NO & YES & NO \\
\hline 1573 & Q00796 & DHSO & 38.32 & $23.50 \%$ & YES & YES & YES & NO \\
\hline 1574 & P08582 & TRFM & 80.21 & $11.50 \%$ & NO & NO & YES & NO \\
\hline 1575 & Q9Y224 & CN166 & 28.07 & $22.10 \%$ & NO & NO & NO & NO \\
\hline 1576 & Q8IZ83 & A16A1 & 85.13 & $11.10 \%$ & NO & YES & NO & NO \\
\hline 1577 & P30085 & $\mathrm{KCY}$ & 22.22 & $24.50 \%$ & YES & YES & YES & NO \\
\hline 1578 & Q9NY33 & DPP3 & 82.59 & $14.70 \%$ & YES & NO & NO & NO \\
\hline 1579 & E7EP00 & E7EP00 & 118.33 & $7.69 \%$ & NO & NO & NO & NO \\
\hline 1580 & J3KSJ0 & J3KRX5 & 21.40 & $20.10 \%$ & NO & YES & NO & NO \\
\hline 1581 & P30419 & NMT1 & 56.81 & $7.46 \%$ & YES & YES & NO & NO \\
\hline 1582 & C9J7E5 & E9PFH4 & 104.20 & $11.70 \%$ & NO & YES & NO & NO \\
\hline 1583 & P62913 & RL11 & 20.25 & $24.20 \%$ & NO & $\mathrm{NO}$ & YES & NO \\
\hline 1584 & Q13045 & FLII & 144.76 & $6.70 \%$ & NO & NO & NO & NO \\
\hline 1585 & P40429 & RL13A & 23.58 & $15.80 \%$ & NO & YES & YES & $\mathrm{NO}$ \\
\hline 1586 & P31689 & DNJA1 & 44.87 & $8.06 \%$ & NO & NO & YES & $\mathrm{NO}$ \\
\hline 1587 & Q14839 & CHD4 & 218.01 & $3.71 \%$ & NO & NO & NO & NO \\
\hline 1588 & P05362 & ICAM1 & 57.82 & $8.08 \%$ & NO & NO & YES & NO \\
\hline 1589 & Q92616 & GCN1L & 292.76 & $3.44 \%$ & NO & NO & YES & $\mathrm{NO}$ \\
\hline 1590 & P42704 & LPPRC & 157.91 & $6.03 \%$ & NO & NO & YES & $\mathrm{NO}$ \\
\hline 1591 & P14735 & IDE & 117.97 & $4.81 \%$ & NO & NO & NO & NO \\
\hline 1592 & Q8TEX9 & IPO4 & 118.72 & $10.70 \%$ & NO & NO & YES & NO \\
\hline 1593 & P42765 & THIM & 41.92 & $11.10 \%$ & NO & $\mathrm{NO}$ & YES & NO \\
\hline 1594 & Q9Y6E2 & BZW2 & 48.16 & $8.11 \%$ & $\mathrm{NO}$ & NO & NO & NO \\
\hline 1595 & Q07065 & CKAP4 & 66.02 & $6.15 \%$ & NO & YES & YES & NO \\
\hline 1596 & Q14683 & SMC1A & 143.24 & $4.06 \%$ & $\mathrm{NO}$ & NO & NO & NO \\
\hline 1597 & Q09666 & AHNK & 629.10 & $0.99 \%$ & NO & $\mathrm{NO}$ & YES & NO \\
\hline 1598 & Q9BT78 & CSN4 & 46.27 & $11.10 \%$ & $\mathrm{NO}$ & YES & NO & NO \\
\hline 1599 & H0YFF6 & E7EPK1 & 50.68 & $19.00 \%$ & NO & NO & NO & NO \\
\hline 1600 & P49458 & SRP09 & 10.11 & $30.20 \%$ & NO & YES & NO & NO \\
\hline 1601 & B4DZI8 & B4DZI8 & 102.49 & $3.88 \%$ & NO & $\mathrm{NO}$ & NO & NO \\
\hline 1602 & Q10570 & CPSF1 & 160.89 & $2.56 \%$ & $\mathrm{NO}$ & NO & NO & NO \\
\hline 1603 & P35637 & FUS & 53.43 & $5.13 \%$ & YES & $\mathrm{NO}$ & NO & NO \\
\hline 1604 & D6RAH7 & D6RAH7 & 16.74 & $24.60 \%$ & NO & YES & NO & $\mathrm{NO}$ \\
\hline 1605 & Q96PD2 & DCBD2 & 85.04 & $14.60 \%$ & NO & $\mathrm{NO}$ & NO & NO \\
\hline 1606 & Q9NRW7 & VPS45 & 65.08 & $4.74 \%$ & NO & NO & NO & NO \\
\hline 1607 & B0QZ18 & B0QZ18 & 59.06 & $5.54 \%$ & NO & NO & NO & $\mathrm{NO}$ \\
\hline 1608 & G5E935 & C9JDE9 & 40.27 & $8.62 \%$ & NO & YES & NO & $\mathrm{NO}$ \\
\hline 1609 & F8W871 & A8MQB8 & 71.17 & $3.78 \%$ & NO & YES & NO & NO \\
\hline 1610 & P54577 & SYYC & 59.15 & $9.09 \%$ & NO & NO & NO & NO \\
\hline 1611 & B4DQ14 & B4DQ14 & 64.99 & $5.89 \%$ & YES & NO & NO & NO \\
\hline 1612 & P35241 & A7YIJ8 & 68.57 & $19.70 \%$ & NO & NO & NO & $\mathrm{NO}$ \\
\hline 1613 & O95428 & PPN & 137.70 & $4.46 \%$ & NO & NO & YES & NO \\
\hline 1614 & H0YDU8 & H0YDU8 & 56.88 & $8.87 \%$ & NO & NO & $\mathrm{NO}$ & $\mathrm{NO}$ \\
\hline 1615 & P04632 & CPNS1 & 28.32 & $12.30 \%$ & NO & YES & YES & $\mathrm{NO}$ \\
\hline 1616 & Q16222 & UAP1 & 58.77 & $5.36 \%$ & NO & YES & NO & NO \\
\hline 1617 & P05161 & ISG15 & 17.89 & $17.60 \%$ & NO & YES & NO & $\mathrm{NO}$ \\
\hline
\end{tabular}




\begin{tabular}{|c|c|c|c|c|c|c|c|c|}
\hline 1618 & J3KN06 & E7ESG3 & 48.15 & $7.98 \%$ & YES & YES & $\mathrm{NO}$ & NO \\
\hline 1619 & B1AKJ5 & B1AKJ5 & 131.58 & $4.43 \%$ & $\mathrm{NO}$ & $\mathrm{NO}$ & $\mathrm{NO}$ & $\mathrm{NO}$ \\
\hline 1620 & G3XAE6 & G3XAE6 & 100.46 & $3.29 \%$ & $\mathrm{NO}$ & $\mathrm{NO}$ & $\mathrm{NO}$ & NO \\
\hline 1621 & O94973 & AP2A2 & 103.96 & $8.31 \%$ & $\mathrm{NO}$ & $\mathrm{NO}$ & YES & NO \\
\hline 1622 & E9PGZ2 & E9PGZ2 & 73.06 & $5.16 \%$ & $\mathrm{NO}$ & YES & $\mathrm{NO}$ & $\mathrm{NO}$ \\
\hline 1623 & Q08378 & GOGA3 & 167.36 & $1.94 \%$ & $\mathrm{NO}$ & $\mathrm{NO}$ & $\mathrm{NO}$ & NO \\
\hline 1624 & B4DDP6 & B4DDP6 & 48.21 & $9.16 \%$ & $\mathrm{NO}$ & YES & $\mathrm{NO}$ & $\mathrm{NO}$ \\
\hline 1625 & A8MUN4 & A8MUN4 & 26.15 & $12.20 \%$ & YES & $\mathrm{NO}$ & $\mathrm{NO}$ & NO \\
\hline 1626 & E5RJT4 & E5RJG0 & 79.29 & $25.00 \%$ & NO & NO & NO & NO \\
\hline 1627 & O75095 & MEGF6 & 161.17 & $3.83 \%$ & $\mathrm{NO}$ & $\mathrm{NO}$ & YES & NO \\
\hline 1628 & Q9Y6D6 & BIG1 & 208.77 & $1.57 \%$ & $\mathrm{NO}$ & $\mathrm{NO}$ & $\mathrm{NO}$ & NO \\
\hline 1629 & Q92888 & ARHG1 & 102.44 & $3.40 \%$ & $\mathrm{NO}$ & $\mathrm{NO}$ & $\mathrm{NO}$ & NO \\
\hline 1630 & P35573 & GDE & 174.77 & $2.55 \%$ & YES & NO & NO & NO \\
\hline 1631 & G3XAL7 & G3XAL7 & 46.70 & $10.30 \%$ & $\mathrm{NO}$ & YES & $\mathrm{NO}$ & NO \\
\hline 1632 & E5RGI1 & F5H0B0 & 31.17 & $10.40 \%$ & $\mathrm{NO}$ & $\mathrm{NO}$ & NO & NO \\
\hline 1633 & Q9UN86 & G3BP2 & 54.12 & $7.88 \%$ & $\mathrm{NO}$ & $\mathrm{NO}$ & $\mathrm{NO}$ & NO \\
\hline 1634 & P53680 & AP2S1 & 17.02 & $14.80 \%$ & YES & $\mathrm{NO}$ & $\mathrm{NO}$ & $\mathrm{NO}$ \\
\hline 1635 & Q6DD88 & ATLA3 & 60.54 & $3.88 \%$ & $\mathrm{NO}$ & YES & $\mathrm{NO}$ & NO \\
\hline 1636 & Q01081 & U2AF1 & 27.87 & $13.30 \%$ & $\mathrm{NO}$ & $\mathrm{NO}$ & YES & NO \\
\hline 1637 & A6NDN0 & A6NDN0 & 20.27 & $15.70 \%$ & $\mathrm{NO}$ & YES & $\mathrm{NO}$ & NO \\
\hline 1638 & Q13617 & CUL2 & 86.99 & $8.59 \%$ & $\mathrm{NO}$ & $\mathrm{NO}$ & $\mathrm{NO}$ & $\mathrm{NO}$ \\
\hline 1639 & Q14CX7 & NAA25 & 112.30 & $4.12 \%$ & $\mathrm{NO}$ & $\mathrm{NO}$ & $\mathrm{NO}$ & NO \\
\hline 1640 & J3KQB0 & J3KQB0 & 39.32 & $6.83 \%$ & $\mathrm{NO}$ & YES & NO & NO \\
\hline 1641 & O00429 & DNM1L & 81.88 & $7.20 \%$ & $\mathrm{NO}$ & $\mathrm{NO}$ & $\mathrm{NO}$ & $\mathrm{NO}$ \\
\hline 1642 & P43121 & MUC18 & 71.61 & $4.18 \%$ & $\mathrm{NO}$ & $\mathrm{NO}$ & YES & $\mathrm{NO}$ \\
\hline 1643 & B4DRZ7 & B4DRZ7 & 53.98 & $7.73 \%$ & $\mathrm{NO}$ & $\mathrm{NO}$ & $\mathrm{NO}$ & $\mathrm{NO}$ \\
\hline 1644 & Н7C0P8 & E7EN38 & 153.90 & $5.45 \%$ & $\mathrm{NO}$ & YES & $\mathrm{NO}$ & NO \\
\hline 1645 & Q9BXS5 & AP1M1 & 48.59 & $7.33 \%$ & $\mathrm{NO}$ & YES & $\mathrm{NO}$ & $\mathrm{NO}$ \\
\hline 1646 & B3KW71 & B3KW71 & 25.79 & $14.10 \%$ & $\mathrm{NO}$ & $\mathrm{NO}$ & $\mathrm{NO}$ & $\mathrm{NO}$ \\
\hline 1647 & Q6DKJ4 & NXN & 48.39 & $6.90 \%$ & YES & $\mathrm{NO}$ & $\mathrm{NO}$ & $\mathrm{NO}$ \\
\hline 1648 & P01137 & TGFB1 & 44.34 & $7.69 \%$ & $\mathrm{NO}$ & $\mathrm{NO}$ & YES & NO \\
\hline 1649 & Q99805 & TM9S2 & 75.78 & $4.37 \%$ & NO & NO & NO & NO \\
\hline 1650 & F8W6L6 & F8VTL3 & 229.01 & $6.48 \%$ & YES & $\mathrm{NO}$ & $\mathrm{NO}$ & NO \\
\hline 1651 & Q15738 & NSDHL & 41.90 & $15.00 \%$ & $\mathrm{NO}$ & $\mathrm{NO}$ & YES & NO \\
\hline 1652 & Q9NR30 & DDX21 & 87.35 & $4.21 \%$ & $\mathrm{NO}$ & YES & YES & NO \\
\hline 1653 & P17931 & LEG3 & 26.15 & $8.80 \%$ & YES & YES & YES & $\mathrm{NO}$ \\
\hline 1654 & Q9Y3L5 & RAP2C & 20.74 & $31.70 \%$ & $\mathrm{NO}$ & YES & YES & NO \\
\hline 1655 & B4DKT0 & B4DKT0 & 47.15 & $6.03 \%$ & $\mathrm{NO}$ & $\mathrm{NO}$ & NO & $\mathrm{NO}$ \\
\hline 1656 & Q96J84 & KIRR1 & 83.54 & $3.83 \%$ & YES & $\mathrm{NO}$ & YES & NO \\
\hline 1657 & Q14694 & UBP10 & 87.13 & $3.76 \%$ & $\mathrm{NO}$ & $\mathrm{NO}$ & $\mathrm{NO}$ & $\mathrm{NO}$ \\
\hline 1658 & Е9РB90 & E9PB90 & 102.38 & $7.31 \%$ & $\mathrm{NO}$ & $\mathrm{NO}$ & $\mathrm{NO}$ & NO \\
\hline 1659 & Q02487 & DSC2 & 99.96 & $4.11 \%$ & YES & $\mathrm{NO}$ & YES & NO \\
\hline 1660 & Q9NX46 & ARHL2 & 38.95 & $6.34 \%$ & $\mathrm{NO}$ & NO & NO & $\mathrm{NO}$ \\
\hline 1661 & Q9UL15 & BAG5 & 51.20 & $7.16 \%$ & $\mathrm{NO}$ & YES & $\mathrm{NO}$ & NO \\
\hline 1662 & Е9РНH9 & Е9РНH9 & 60.61 & $5.84 \%$ & $\mathrm{NO}$ & $\mathrm{NO}$ & $\mathrm{NO}$ & $\mathrm{NO}$ \\
\hline 1663 & H3BR38 & H3BM50 & 35.88 & $19.80 \%$ & $\mathrm{NO}$ & YES & $\mathrm{NO}$ & NO \\
\hline 1664 & Q9BTV4 & TMM43 & 44.88 & $4.75 \%$ & NO & YES & NO & NO \\
\hline 1665 & P30837 & AL1B1 & 57.21 & $6.77 \%$ & $\mathrm{NO}$ & YES & NO & NO \\
\hline 1666 & Q9HAV4 & XPO5 & 136.31 & $1.91 \%$ & $\mathrm{NO}$ & NO & NO & $\mathrm{NO}$ \\
\hline 1667 & F8VYA6 & F5GYK2 & 80.60 & $4.73 \%$ & $\mathrm{NO}$ & $\mathrm{NO}$ & $\mathrm{NO}$ & NO \\
\hline 1668 & F8WBK9 & C9JIG9 & 58.02 & $4.91 \%$ & YES & $\mathrm{NO}$ & $\mathrm{NO}$ & $\mathrm{NO}$ \\
\hline 1669 & Q9H3H3 & CK068 & 27.35 & $8.76 \%$ & $\mathrm{NO}$ & YES & $\mathrm{NO}$ & NO \\
\hline 1670 & P18031 & PTN1 & 49.97 & $5.29 \%$ & $\mathrm{NO}$ & $\mathrm{NO}$ & $\mathrm{NO}$ & NO \\
\hline 1671 & Q8NHH9 & ATLA2 & 64.52 & $5.83 \%$ & $\mathrm{NO}$ & YES & NO & NO \\
\hline 1672 & Q9NVX2 & NLE1 & 53.32 & $5.98 \%$ & $\mathrm{NO}$ & $\mathrm{NO}$ & $\mathrm{NO}$ & NO \\
\hline 1673 & Q92879 & CELF1 & 52.06 & $4.73 \%$ & YES & $\mathrm{NO}$ & $\mathrm{NO}$ & NO \\
\hline
\end{tabular}




\begin{tabular}{|c|c|c|c|c|c|c|c|c|c|}
\hline 1674 & D6RA13 & E9PF10 & 155.20 & $1.43 \%$ & NO & YES & $\mathrm{NO}$ & NO & $\mathrm{NO}$ \\
\hline 1675 & Q8N8S7 & ENAH & 66.51 & $4.23 \%$ & YES & $\mathrm{NO}$ & $\mathrm{NO}$ & $\mathrm{NO}$ & $\mathrm{NO}$ \\
\hline 1676 & P61221 & ABCE1 & 67.32 & $4.34 \%$ & NO & $\mathrm{NO}$ & $\mathrm{NO}$ & NO & $\mathrm{NO}$ \\
\hline 1677 & Q86UD1 & OAF & 30.69 & $42.10 \%$ & NO & NO & YES & NO & $\mathrm{NO}$ \\
\hline 1678 & Q13283 & G3BP1 & 52.16 & $32.00 \%$ & NO & NO & YES & $\mathrm{NO}$ & $\mathrm{NO}$ \\
\hline 1679 & Q00839 & HNRPU & 90.59 & $13.90 \%$ & NO & NO & YES & NO & $\mathrm{NO}$ \\
\hline 1680 & Q9Y5B9 & SP16H & 119.92 & $8.69 \%$ & NO & NO & $\mathrm{NO}$ & NO & $\mathrm{NO}$ \\
\hline 1681 & Q9UMS4 & PRP19 & 55.18 & $22.00 \%$ & NO & NO & NO & NO & $\mathrm{NO}$ \\
\hline 1682 & O43592 & XPOT & 109.97 & $7.17 \%$ & NO & YES & $\mathrm{NO}$ & NO & $\mathrm{NO}$ \\
\hline 1683 & P49721 & PSB2 & 22.84 & $14.40 \%$ & NO & NO & YES & NO & $\mathrm{NO}$ \\
\hline 1684 & F8VUA6 & F8VUA6 & 21.64 & $28.50 \%$ & YES & YES & $\mathrm{NO}$ & NO & $\mathrm{NO}$ \\
\hline 1685 & P00390 & GSHR & 56.26 & $15.10 \%$ & NO & YES & YES & NO & $\mathrm{NO}$ \\
\hline 1686 & A8MUD9 & A8MUD9 & 29.23 & $32.70 \%$ & YES & YES & NO & NO & $\mathrm{NO}$ \\
\hline 1687 & B4DPJ6 & B4DPJ6 & 22.24 & $14.70 \%$ & NO & YES & NO & NO & $\mathrm{NO}$ \\
\hline 1688 & B4DT31 & B4DT31 & 67.56 & $6.02 \%$ & NO & NO & NO & NO & $\mathrm{NO}$ \\
\hline 1689 & Q9Y3U8 & RL36 & 12.25 & $24.80 \%$ & YES & YES & NO & NO & $\mathrm{NO}$ \\
\hline 1690 & A8CZ64 & A8CZ64 & 41.39 & $15.50 \%$ & NO & NO & NO & NO & $\mathrm{NO}$ \\
\hline 1691 & B7WPD3 & B7WPD3 & 13.13 & $21.10 \%$ & YES & YES & NO & NO & $\mathrm{NO}$ \\
\hline 1692 & B4DR80 & B4DR80 & 49.31 & $13.10 \%$ & YES & YES & NO & NO & $\mathrm{NO}$ \\
\hline 1693 & Q96PK6 & RBM14 & 69.49 & $3.59 \%$ & NO & NO & NO & NO & $\mathrm{NO}$ \\
\hline 1694 & Q9H3K6 & BOLA2 & 10.12 & $30.20 \%$ & NO & YES & YES & NO & $\mathrm{NO}$ \\
\hline 1695 & Q9UBV8 & PEF1 & 30.38 & $8.45 \%$ & NO & YES & YES & NO & $\mathrm{NO}$ \\
\hline 1696 & Q9P035 & HACD3 & 43.16 & $8.84 \%$ & NO & NO & NO & NO & $\mathrm{NO}$ \\
\hline 1697 & P37173 & TGFR2 & 64.57 & $3.88 \%$ & NO & NO & YES & NO & $\mathrm{NO}$ \\
\hline 1698 & Q9UHD8 & SEPT9 & 65.40 & $15.50 \%$ & NO & YES & NO & NO & $\mathrm{NO}$ \\
\hline 1699 & O15143 & ARC1B & 40.95 & $7.53 \%$ & YES & $\mathrm{NO}$ & $\mathrm{NO}$ & $\mathrm{NO}$ & $\mathrm{NO}$ \\
\hline 1700 & E9PDF6 & E9PDF6 & 131.99 & $2.89 \%$ & NO & NO & NO & NO & $\mathrm{NO}$ \\
\hline 1701 & Q9H6S3 & ES8L2 & 80.62 & $3.92 \%$ & NO & NO & YES & NO & $\mathrm{NO}$ \\
\hline 1702 & E7EPS8 & E7EPS8 & 163.68 & $1.22 \%$ & NO & NO & NO & NO & $\mathrm{NO}$ \\
\hline 1703 & Q8NFL0 & B3GN7 & 45.99 & $6.73 \%$ & NO & NO & NO & NO & $\mathrm{NO}$ \\
\hline 1704 & O60841 & IF2P & 138.83 & $3.11 \%$ & NO & NO & NO & NO & $\mathrm{NO}$ \\
\hline 1705 & H3BRY4 & Н3ВТН8 & 40.89 & $11.80 \%$ & YES & NO & NO & NO & $\mathrm{NO}$ \\
\hline 1706 & Q92905 & CSN5 & 37.58 & $6.59 \%$ & YES & NO & NO & NO & $\mathrm{NO}$ \\
\hline 1707 & B4E321 & B4E321 & 75.56 & $11.80 \%$ & NO & NO & $\mathrm{NO}$ & NO & $\mathrm{NO}$ \\
\hline 1708 & Q9Y295 & DRG1 & 40.54 & $8.17 \%$ & NO & NO & NO & NO & $\mathrm{NO}$ \\
\hline 1709 & Q9Y219 & JAG2 & 133.36 & $2.34 \%$ & NO & NO & $\mathrm{NO}$ & $\mathrm{NO}$ & $\mathrm{NO}$ \\
\hline
\end{tabular}


Table S6. 724 differential proteins quantified by 2DE and/or iTRAQ.

\begin{tabular}{|c|c|c|c|c|c|c|}
\hline NO. & $\begin{array}{l}\text { Swiss-prot } \\
\text { Number }\end{array}$ & $\begin{array}{l}\text { Protein } \\
\text { name }\end{array}$ & $\begin{array}{l}\text { classical secreted } \\
\text { proteins }\end{array}$ & $\begin{array}{l}\text { non-classical } \\
\text { secreted } \\
\text { proteins }\end{array}$ & urinary proteins & $\begin{array}{l}\text { Analysis } \\
\text { resource }\end{array}$ \\
\hline 1 & P62736 & ACTA & & & & $2 \mathrm{DE}$ \\
\hline 2 & P02768 & ALBU & NO & NO & NO & $2 \mathrm{DE}$ \\
\hline 3 & P07339 & CATD & YES & NO & YES & $2 \mathrm{DE}$ \\
\hline 4 & P46087 & NOP2 & YES & NO & YES & 2DE \& iTRAQ \\
\hline 5 & P22626 & ROA2 & NO & YES & NO & $2 \mathrm{DE}$ \\
\hline 6 & Q13885 & TBB2A & NO & NO & YES & $2 \mathrm{DE}$ \\
\hline 7 & Q18PE1 & DOK7 & NO & NO & NO & 2DE \\
\hline 8 & P31947 & $1433 \mathrm{~S}$ & NO & NO & NO & 2DE \& iTRAQ \\
\hline 9 & O95336 & 6PGL & NO & NO & $\mathrm{NO}$ & 2DE \& iTRAQ \\
\hline 10 & P05091 & ALDH2 & NO & YES & YES & $2 \mathrm{DE}$ \\
\hline 11 & P15121 & ALDR & NO & YES & NO & 2DE \& iTRAQ \\
\hline 12 & P39687 & AN32A & NO & NO & YES & $2 \mathrm{DE}$ \\
\hline 13 & P05089 & ARGI1 & NO & NO & YES & $2 \mathrm{DE}$ \\
\hline 14 & P10809 & CH60 & NO & NO & YES & $2 \mathrm{DE}$ \\
\hline 15 & P00167 & CYB5 & NO & NO & YES & $2 \mathrm{DE}$ \\
\hline 16 & P16930 & FAAA & NO & NO & YES & 2DE \\
\hline 17 & Q13283 & G3BP1 & NO & NO & YES & $2 \mathrm{DE}$ \\
\hline 18 & Q96QV6 & H2A1A & NO & NO & YES & $2 \mathrm{DE}$ \\
\hline 19 & Q96A08 & H2B1A & NO & NO & NO & $2 \mathrm{DE}$ \\
\hline 20 & P62805 & H4 & NO & NO & NO & 2DE \& iTRAQ \\
\hline 21 & P04792 & HSPB1 & NO & NO & YES & 2DE \& iTRAQ \\
\hline 22 & P14618 & KPYM & NO & YES & YES & 2DE \\
\hline 23 & P40925 & MDHC & NO & NO & YES & $2 \mathrm{DE}$ \\
\hline 24 & P14174 & MIF & NO & NO & YES & 2DE \\
\hline 25 & P06748 & NPM & NO & YES & YES & $2 \mathrm{DE}$ \\
\hline 26 & P61970 & NTF2 & NO & YES & NO & 2DE \& iTRAQ \\
\hline 27 & Q99497 & PARK7 & NO & YES & YES & 2DE \& iTRAQ \\
\hline 28 & P12004 & PCNA & NO & NO & YES & $2 \mathrm{DE}$ \\
\hline 29 & P30101 & PDIA3 & NO & NO & NO & $2 \mathrm{DE}$ \\
\hline 30 & P30086 & PEBP1 & YES & NO & YES & 2DE \\
\hline 31 & P18669 & PGAM1 & NO & YES & YES & $2 \mathrm{DE}$ \\
\hline 32 & P30048 & PRDX3 & NO & NO & YES & $2 \mathrm{DE}$ \\
\hline 33 & P30041 & PRDX6 & NO & YES & YES & $2 \mathrm{DE}$ \\
\hline 34 & P28066 & PSA5 & NO & NO & YES & 2DE \\
\hline 35 & P49721 & PSB2 & NO & NO & YES & $2 \mathrm{DE}$ \\
\hline 36 & P49720 & PSB3 & NO & NO & YES & 2DE \& iTRAQ \\
\hline 37 & P28070 & PSB4 & $\mathrm{NO}$ & NO & YES & $2 \mathrm{DE}$ \\
\hline 38 & P28074 & PSB5 & $\mathrm{NO}$ & YES & YES & 2DE \\
\hline 39 & P28072 & PSB6 & NO & YES & YES & 2DE \\
\hline 40 & Q99436 & PSB7 & NO & NO & YES & $2 \mathrm{DE}$ \\
\hline 41 & Q32P51 & RA1L2 & NO & NO & YES & $2 \mathrm{DE}$ \\
\hline 42 & P62306 & RUXF & $\mathrm{NO}$ & NO & NO & 2DE \\
\hline 43 & Q01105 & SET & $\mathrm{NO}$ & NO & NO & $2 \mathrm{DE}$ \\
\hline 44 & P09486 & SPARC & NO & NO & NO & 2DE \& iTRAQ \\
\hline 45 & P36952 & SPB5 & YES & NO & YES & 2DE \& iTRAQ \\
\hline 46 & Q04837 & SSBP & $\mathrm{NO}$ & NO & YES & 2DE \\
\hline 47 & P06753 & TPM3 & NO & YES & NO & 2DE \\
\hline 48 & Q9UJN7 & ZN391 & NO & NO & YES & $2 \mathrm{DE}$ \\
\hline 49 & P61769 & B2MG & NO & YES & NO & 2DE \& iTRAQ \\
\hline 50 & P23528 & COF1 & YES & NO & YES & 2DE \\
\hline
\end{tabular}




\begin{tabular}{|c|c|c|c|c|c|c|}
\hline 51 & P02751 & FINC & NO & YES & $\mathrm{NO}$ & 2DE \& iTRAQ \\
\hline 52 & P40926 & MDHM & YES & NO & YES & $2 \mathrm{DE}$ \\
\hline 53 & P60174 & TPIS & $\mathrm{NO}$ & YES & YES & 2DE \& iTRAQ \\
\hline 54 & Q30KQ2 & DB130 & NO & NO & YES & $2 \mathrm{DE}$ \\
\hline 55 & P33778 & H2B1B & YES & NO & NO & $2 \mathrm{DE}$ \\
\hline 56 & P11047 & LAMC1 & NO & NO & NO & 2DE \& iTRAQ \\
\hline 57 & P08253 & MMP2 & YES & NO & YES & 2DE \& iTRAQ \\
\hline 58 & P80188 & NGAL & YES & NO & YES & $2 \mathrm{DE}$ \\
\hline 59 & P98160 & PGBM & YES & NO & YES & 2DE \& iTRAQ \\
\hline 60 & P32119 & PRDX2 & YES & NO & YES & $2 \mathrm{DE}$ \\
\hline 61 & Q15223 & PVRL1 & NO & NO & YES & $2 \mathrm{DE}$ \\
\hline 62 & P00441 & SODC & YES & NO & YES & $2 \mathrm{DE}$ \\
\hline 63 & P40763 & STAT3 & NO & YES & YES & $2 \mathrm{DE}$ \\
\hline 64 & Q9NU19 & TB22B & $\mathrm{NO}$ & NO & YES & $2 \mathrm{DE}$ \\
\hline 65 & Q9GZM7 & TINAL & $\mathrm{NO}$ & NO & $\mathrm{NO}$ & 2DE \& iTRAQ \\
\hline 66 & P07996 & TSP1 & YES & NO & YES & 2DE \& iTRAQ \\
\hline 67 & Q8N2E2 & VWDE & YES & NO & YES & $2 \mathrm{DE}$ \\
\hline 68 & P04075 & ALDOA & YES & NO & $\mathrm{NO}$ & 2DE \& iTRAQ \\
\hline 69 & Q92876 & KLK6 & $\mathrm{NO}$ & NO & YES & 2DE \& iTRAQ \\
\hline 70 & P00558 & PGK1 & YES & NO & YES & 2DE \& iTRAQ \\
\hline 71 & P61457 & PHS & $\mathrm{NO}$ & NO & YES & $2 \mathrm{DE}$ \\
\hline 72 & P07737 & PROF1 & NO & NO & YES & 2DE \& iTRAQ \\
\hline 73 & P06733 & ENOA & $\mathrm{NO}$ & NO & YES & $2 \mathrm{DE}$ \\
\hline 74 & P09211 & GSTP1 & NO & NO & YES & $2 \mathrm{DE}$ \\
\hline 75 & P09382 & LEG1 & $\mathrm{NO}$ & NO & YES & 2DE \& iTRAQ \\
\hline 76 & Q12912 & LRMP & $\mathrm{NO}$ & NO & YES & $2 \mathrm{DE}$ \\
\hline 77 & P15531 & NDKA & NO & YES & $\mathrm{NO}$ & $2 \mathrm{DE}$ \\
\hline 78 & P05067 & A4 & NO & NO & $\mathrm{NO}$ & 2DE \& iTRAQ \\
\hline 79 & O00468 & AGRIN & YES & NO & YES & 2DE \& iTRAQ \\
\hline 80 & P01008 & ANT3 & YES & NO & YES & 2DE \\
\hline 81 & P02649 & APOE & YES & NO & YES & $2 \mathrm{DE}$ \\
\hline 82 & P01034 & CYTC & YES & NO & YES & 2DE \& iTRAQ \\
\hline 83 & Q15392 & DHC24 & YES & NO & YES & $2 \mathrm{DE}$ \\
\hline 84 & P04406 & G3P & YES & NO & $\mathrm{NO}$ & 2DE \& iTRAQ \\
\hline 85 & P78417 & GSTO1 & NO & NO & YES & $2 \mathrm{DE}$ \\
\hline 86 & P68871 & HBB & NO & NO & YES & $2 \mathrm{DE}$ \\
\hline 87 & P12277 & KCRB & NO & NO & YES & $2 \mathrm{DE}$ \\
\hline 88 & P06732 & KCRM & NO & NO & YES & 2DE \\
\hline 89 & Q96G25 & MED8 & NO & NO & $\mathrm{NO}$ & $2 \mathrm{DE}$ \\
\hline 90 & P05121 & PAI1 & NO & NO & $\mathrm{NO}$ & 2DE \& iTRAQ \\
\hline 91 & Q9UNA4 & POLI & YES & NO & YES & $2 \mathrm{DE}$ \\
\hline 92 & P27169 & PON1 & NO & NO & $\mathrm{NO}$ & $2 \mathrm{DE}$ \\
\hline 93 & P60900 & PSA6 & YES & NO & YES & $2 \mathrm{DE}$ \\
\hline 94 & O00560 & SDCB1 & NO & NO & YES & $2 \mathrm{DE}$ \\
\hline 95 & Q9P273 & TEN3 & NO & NO & YES & 2DE \\
\hline 96 & P01033 & TIMP1 & NO & NO & $\mathrm{NO}$ & $2 \mathrm{DE}$ \\
\hline 97 & P16035 & TIMP2 & YES & NO & YES & 2DE \\
\hline 98 & O00755 & WNT7A & YES & NO & YES & $2 \mathrm{DE}$ \\
\hline 99 & P04040 & CATA & YES & NO & $\mathrm{NO}$ & $2 \mathrm{DE}$ \\
\hline 100 & Р07237 & PDIA1 & NO & NO & YES & $2 \mathrm{DE}$ \\
\hline 101 & P25787 & PSA2 & YES & NO & YES & $2 \mathrm{DE}$ \\
\hline 102 & P37802 & TAGL2 & NO & NO & YES & $2 \mathrm{DE}$ \\
\hline 103 & P13693 & ТСТР & $\mathrm{NO}$ & YES & YES & $2 \mathrm{DE}$ \\
\hline 104 & P00338 & LDHA & NO & NO & YES & iTRAQ \\
\hline 105 & Р07093 & GDN & YES & YES & $\mathrm{NO}$ & iTRAQ \\
\hline 106 & P25391 & LAMA1 & $\mathrm{NO}$ & NO & $\mathrm{NO}$ & iTRAQ \\
\hline
\end{tabular}




\begin{tabular}{|c|c|c|c|c|c|c|}
\hline 107 & P06744 & G6PI & $\mathrm{NO}$ & NO & NO & iTRAQ \\
\hline 108 & P01009 & $\mathrm{A} 1 \mathrm{AT}$ & $\mathrm{NO}$ & NO & YES & iTRAQ \\
\hline 109 & P49788 & TIG1 & $\mathrm{NO}$ & NO & YES & iTRAQ \\
\hline 110 & B4DDD8 & B3KWE1 & YES & NO & YES & iTRAQ \\
\hline 111 & O00151 & PDLI1 & YES & NO & NO & iTRAQ \\
\hline 112 & P13928 & ANXA8 & $\mathrm{NO}$ & NO & YES & iTRAQ \\
\hline 113 & E9PF66 & F5H1G4 & $\mathrm{NO}$ & NO & YES & iTRAQ \\
\hline 114 & С9JXX7 & C9JK10 & YES & NO & NO & iTRAQ \\
\hline 115 & P61225 & RAP2B & YES & YES & NO & iTRAQ \\
\hline 116 & O43240 & KLK10 & $\mathrm{NO}$ & YES & YES & iTRAQ \\
\hline 117 & P12830 & CADH1 & YES & NO & YES & iTRAQ \\
\hline 118 & B4DTU4 & B4DTU4 & $\mathrm{NO}$ & NO & YES & iTRAQ \\
\hline 119 & Q6EMK4 & VASN & YES & NO & NO & iTRAQ \\
\hline 120 & P32320 & CDD & $\mathrm{NO}$ & NO & YES & iTRAQ \\
\hline 121 & P49862 & KLK7 & $\mathrm{NO}$ & YES & YES & iTRAQ \\
\hline 122 & H9KV70 & H9KV70 & $\mathrm{NO}$ & NO & NO & iTRAQ \\
\hline 123 & Q99715 & COCA1 & $\mathrm{NO}$ & NO & NO & iTRAQ \\
\hline 124 & P67936 & TPM4 & YES & NO & YES & iTRAQ \\
\hline 125 & Q16363 & LAMA4 & $\mathrm{NO}$ & NO & NO & iTRAQ \\
\hline 126 & P08476 & INHBA & $\mathrm{NO}$ & NO & YES & iTRAQ \\
\hline 127 & Q96KP4 & CNDP2 & $\mathrm{NO}$ & NO & NO & iTRAQ \\
\hline 128 & P61158 & ARP3 & YES & NO & YES & iTRAQ \\
\hline 129 & Q16394 & EXT1 & $\mathrm{NO}$ & NO & YES & iTRAQ \\
\hline 130 & P09341 & GROA & $\mathrm{NO}$ & NO & NO & iTRAQ \\
\hline 131 & P42785 & PCP & $\mathrm{NO}$ & NO & YES & iTRAQ \\
\hline 132 & P02462 & CO4A1 & $\mathrm{NO}$ & NO & YES & iTRAQ \\
\hline 133 & O60763 & USO1 & YES & NO & YES & iTRAQ \\
\hline 134 & O14672 & ADA10 & $\mathrm{NO}$ & NO & YES & iTRAQ \\
\hline 135 & P45452 & MMP13 & YES & NO & YES & iTRAQ \\
\hline 136 & P61812 & TGFB2 & YES & NO & NO & iTRAQ \\
\hline 137 & B0S872 & B0S872 & $\mathrm{NO}$ & NO & NO & iTRAQ \\
\hline 138 & Q9UKP4 & ATS7 & $\mathrm{NO}$ & NO & NO & iTRAQ \\
\hline 139 & F5GWS3 & F5GWS3 & $\mathrm{NO}$ & NO & NO & iTRAQ \\
\hline 140 & O43405 & $\mathrm{COCH}$ & YES & YES & NO & iTRAQ \\
\hline 141 & B3KSQ1 & B3KSQ1 & YES & NO & YES & iTRAQ \\
\hline 142 & Q01484 & ANK2 & $\mathrm{NO}$ & NO & NO & iTRAQ \\
\hline 143 & O15230 & LAMA5 & $\mathrm{NO}$ & NO & NO & iTRAQ \\
\hline 144 & G3XAI2 & G3XAI2 & YES & NO & YES & iTRAQ \\
\hline 145 & O75369 & FLNB & YES & NO & NO & iTRAQ \\
\hline 146 & P53634 & CATC & $\mathrm{NO}$ & NO & YES & iTRAQ \\
\hline 147 & Q02388 & CO7A1 & $\mathrm{NO}$ & NO & YES & iTRAQ \\
\hline 148 & B4DR52 & B4DR52 & $\mathrm{NO}$ & NO & YES & iTRAQ \\
\hline 149 & E7ET40 & E7ET40 & $\mathrm{NO}$ & YES & NO & iTRAQ \\
\hline 150 & P13796 & PLSL & YES & NO & NO & iTRAQ \\
\hline 151 & P08581 & MET & YES & YES & YES & iTRAQ \\
\hline 152 & Q14112 & NID2 & $\mathrm{NO}$ & NO & NO & iTRAQ \\
\hline 153 & P12956 & XRCC6 & $\mathrm{NO}$ & NO & YES & iTRAQ \\
\hline 154 & P35052 & GPC1 & YES & NO & NO & iTRAQ \\
\hline 155 & P00750 & TPA & YES & NO & YES & iTRAQ \\
\hline 156 & Q9HB40 & RISC & $\mathrm{NO}$ & NO & NO & iTRAQ \\
\hline 157 & Q99538 & LGMN & YES & NO & YES & iTRAQ \\
\hline 158 & P00736 & $\mathrm{C} 1 \mathrm{R}$ & $\mathrm{NO}$ & NO & YES & iTRAQ \\
\hline 159 & P11717 & MPRI & $\mathrm{NO}$ & NO & NO & iTRAQ \\
\hline 160 & B7ZMG0 & B7ZMG0 & YES & NO & YES & iTRAQ \\
\hline 161 & Q71UI9 & $\mathrm{H} 2 \mathrm{AV}$ & $\mathrm{NO}$ & NO & NO & iTRAQ \\
\hline 162 & Q9BTY2 & FUCO2 & YES & YES & YES & iTRAQ \\
\hline
\end{tabular}




\begin{tabular}{|c|c|c|c|c|c|c|}
\hline 163 & P46782 & RS5 & $\mathrm{NO}$ & NO & YES & iTRAQ \\
\hline 164 & P24534 & EF1B & $\mathrm{NO}$ & YES & YES & iTRAQ \\
\hline 165 & Е9РЕР6 & Е9PEP6 & $\mathrm{NO}$ & YES & NO & iTRAQ \\
\hline 166 & O00462 & MANBA & $\mathrm{NO}$ & NO & NO & iTRAQ \\
\hline 167 & B9ZVX0 & B9ZVX0 & $\mathrm{NO}$ & NO & YES & iTRAQ \\
\hline 168 & Q05639 & EF1A2 & $\mathrm{NO}$ & NO & NO & iTRAQ \\
\hline 169 & P62081 & RS7 & YES & NO & YES & iTRAQ \\
\hline 170 & B4E2S7 & B4E2S7 & YES & YES & YES & iTRAQ \\
\hline 171 & P55263 & ADK & YES & NO & NO & iTRAQ \\
\hline 172 & P31942 & HNRH3 & $\mathrm{NO}$ & NO & NO & iTRAQ \\
\hline 173 & P16144 & ITB4 & YES & NO & NO & iTRAQ \\
\hline 174 & Q14508 & WFDC2 & $\mathrm{NO}$ & NO & NO & iTRAQ \\
\hline 175 & P14314 & GLU2B & $\mathrm{NO}$ & NO & YES & iTRAQ \\
\hline 176 & A8MUB1 & A8MUB1 & $\mathrm{NO}$ & NO & YES & iTRAQ \\
\hline 177 & H3BLZ8 & C9JMU5 & YES & YES & NO & iTRAQ \\
\hline 178 & B7ZBP9 & A6PVN5 & $\mathrm{NO}$ & NO & NO & iTRAQ \\
\hline 179 & Q14517 & FAT1 & $\mathrm{NO}$ & YES & NO & iTRAQ \\
\hline 180 & Q16719 & KYNU & $\mathrm{NO}$ & NO & NO & iTRAQ \\
\hline 181 & P78539 & SRPX & $\mathrm{NO}$ & NO & NO & iTRAQ \\
\hline 182 & F6U211 & F6U211 & $\mathrm{NO}$ & NO & YES & iTRAQ \\
\hline 183 & P04439 & $1 \mathrm{~A} 03$ & $\mathrm{NO}$ & YES & NO & iTRAQ \\
\hline 184 & P09529 & INHBB & $\mathrm{NO}$ & NO & NO & iTRAQ \\
\hline 185 & P04066 & FUCO & YES & NO & NO & iTRAQ \\
\hline 186 & P00966 & ASSY & $\mathrm{NO}$ & NO & YES & iTRAQ \\
\hline 187 & O75367 & H2AY & $\mathrm{NO}$ & NO & YES & iTRAQ \\
\hline 188 & P30046 & DOPD & YES & NO & YES & iTRAQ \\
\hline 189 & E7EPK6 & E7ЕPK6 & $\mathrm{NO}$ & NO & YES & iTRAQ \\
\hline 190 & Q9NRA1 & PDGFC & YES & YES & NO & iTRAQ \\
\hline 191 & P55145 & MANF & $\mathrm{NO}$ & NO & YES & iTRAQ \\
\hline 192 & P05187 & PPB1 & YES & NO & YES & iTRAQ \\
\hline 193 & P46063 & RECQ1 & $\mathrm{NO}$ & NO & YES & iTRAQ \\
\hline 194 & P39019 & RS19 & YES & NO & NO & iTRAQ \\
\hline 195 & H0YK46 & H0YK46 & YES & YES & YES & iTRAQ \\
\hline 196 & G5E9Y2 & G5E9Y2 & $\mathrm{NO}$ & NO & NO & iTRAQ \\
\hline 197 & E9PHD4 & A6NDW3 & $\mathrm{NO}$ & YES & NO & iTRAQ \\
\hline 198 & Q9NR45 & SIAS & $\mathrm{NO}$ & NO & NO & iTRAQ \\
\hline 199 & Q9Y337 & KLK5 & YES & YES & NO & iTRAQ \\
\hline 200 & A2BHY4 & A2BHY4 & YES & NO & NO & iTRAQ \\
\hline 201 & O75351 & VPS4B & $\mathrm{NO}$ & NO & YES & iTRAQ \\
\hline 202 & F5GWE5 & F5GWE5 & $\mathrm{NO}$ & YES & YES & iTRAQ \\
\hline 203 & A6NDG6 & PGP & $\mathrm{NO}$ & NO & NO & iTRAQ \\
\hline 204 & Q9BRA2 & TXD17 & $\mathrm{NO}$ & NO & YES & iTRAQ \\
\hline 205 & B7ZC38 & B7ZC38 & $\mathrm{NO}$ & NO & YES & iTRAQ \\
\hline 206 & P21291 & CSRP1 & $\mathrm{NO}$ & NO & NO & iTRAQ \\
\hline 207 & O00425 & IF2B3 & $\mathrm{NO}$ & NO & NO & iTRAQ \\
\hline 208 & Q96CS3 & FAF2 & $\mathrm{NO}$ & NO & NO & iTRAQ \\
\hline 209 & B4E2C0 & B4E2C0 & $\mathrm{NO}$ & YES & NO & iTRAQ \\
\hline 210 & P16401 & H15 & $\mathrm{NO}$ & NO & NO & iTRAQ \\
\hline 211 & Q4G148 & GXLT1 & NO & NO & YES & iTRAQ \\
\hline 212 & Q15828 & CYTM & $\mathrm{NO}$ & NO & NO & iTRAQ \\
\hline 213 & P01024 & $\mathrm{CO} 3$ & $\mathrm{NO}$ & NO & YES & iTRAQ \\
\hline 214 & F8W6I7 & F8VRQ1 & $\mathrm{NO}$ & NO & YES & iTRAQ \\
\hline 215 & P24821 & TENA & $\mathrm{NO}$ & NO & NO & iTRAQ \\
\hline 216 & P04264 & $\mathrm{K} 2 \mathrm{C} 1$ & YES & NO & YES & iTRAQ \\
\hline 217 & P04083 & ANXA1 & $\mathrm{NO}$ & NO & YES & iTRAQ \\
\hline 218 & Q92820 & GGH & $\mathrm{NO}$ & YES & YES & iTRAQ \\
\hline
\end{tabular}




\begin{tabular}{|c|c|c|c|c|c|c|}
\hline 219 & P14543 & NID1 & $\mathrm{NO}$ & NO & YES & iTRAQ \\
\hline 220 & P03956 & MMP1 & $\mathrm{NO}$ & NO & YES & iTRAQ \\
\hline 221 & P08572 & $\mathrm{CO} 4 \mathrm{~A} 2$ & $\mathrm{NO}$ & NO & YES & iTRAQ \\
\hline 222 & P23142 & FBLN1 & YES & NO & YES & iTRAQ \\
\hline 223 & P27797 & CALR & $\mathrm{NO}$ & NO & YES & iTRAQ \\
\hline 224 & P06576 & АТРB & YES & NO & YES & iTRAQ \\
\hline 225 & P09238 & MMP10 & $\mathrm{NO}$ & YES & YES & iTRAQ \\
\hline 226 & Q16658 & FSCN1 & NO & NO & YES & iTRAQ \\
\hline 227 & P13010 & XRCC5 & YES & NO & NO & iTRAQ \\
\hline 228 & F5H7N9 & F5H7N9 & $\mathrm{NO}$ & NO & NO & iTRAQ \\
\hline 229 & G3V1S6 & G3V1S6 & $\mathrm{NO}$ & NO & NO & iTRAQ \\
\hline 230 & Q9UI42 & CBPA4 & NO & NO & NO & iTRAQ \\
\hline 231 & P35527 & K1C9 & $\mathrm{NO}$ & NO & YES & iTRAQ \\
\hline 232 & P17936 & IBP3 & YES & NO & YES & iTRAQ \\
\hline 233 & P09668 & CATH & $\mathrm{NO}$ & NO & YES & iTRAQ \\
\hline 234 & P20908 & CO5A1 & $\mathrm{NO}$ & NO & YES & iTRAQ \\
\hline 235 & B1AHL2 & B1AHL2 & $\mathrm{NO}$ & NO & YES & iTRAQ \\
\hline 236 & P54136 & SYRC & $\mathrm{NO}$ & NO & NO & iTRAQ \\
\hline 237 & Q93063 & EXT2 & YES & NO & YES & iTRAQ \\
\hline 238 & Q8IXL6 & DMP4 & YES & YES & NO & iTRAQ \\
\hline 239 & O95782 & AP2A1 & $\mathrm{NO}$ & NO & NO & iTRAQ \\
\hline 240 & P46777 & RL5 & YES & NO & NO & iTRAQ \\
\hline 241 & Q96RW7 & HMCN1 & $\mathrm{NO}$ & NO & YES & iTRAQ \\
\hline 242 & P35908 & K22E & $\mathrm{NO}$ & NO & YES & iTRAQ \\
\hline 243 & B3KS31 & B3KS31 & $\mathrm{NO}$ & NO & YES & iTRAQ \\
\hline 244 & Q8NES3 & LFNG & YES & YES & NO & iTRAQ \\
\hline 245 & B0YIW6 & B0YIW6 & $\mathrm{NO}$ & NO & YES & iTRAQ \\
\hline 246 & C9JFR7 & C9JFR7 & $\mathrm{NO}$ & NO & NO & iTRAQ \\
\hline 247 & Q9BZM5 & N2DL2 & $\mathrm{NO}$ & NO & NO & iTRAQ \\
\hline 248 & P02538 & K2C6A & $\mathrm{NO}$ & NO & NO & iTRAQ \\
\hline 249 & P62280 & RS11 & YES & NO & NO & ITRAQ \\
\hline 250 & Q9UBQ0 & VPS29 & $\mathrm{NO}$ & YES & NO & iTRAQ \\
\hline 251 & B4DUC8 & B4DUC8 & YES & NO & NO & iTRAQ \\
\hline 252 & P21796 & VDAC1 & $\mathrm{NO}$ & YES & NO & iTRAQ \\
\hline 253 & Q24JP5 & T132A & $\mathrm{NO}$ & NO & YES & iTRAQ \\
\hline 254 & P62277 & RS13 & $\mathrm{NO}$ & NO & YES & iTRAQ \\
\hline 255 & B4DER1 & B4DER1 & $\mathrm{NO}$ & YES & YES & ITRAQ \\
\hline 256 & Q7Z304 & MAMC2 & $\mathrm{NO}$ & NO & NO & iTRAQ \\
\hline 257 & F2Z2Y0 & F5H1Z7 & $\mathrm{NO}$ & NO & NO & ITRAQ \\
\hline 258 & P42830 & CXCL5 & YES & NO & NO & iTRAQ \\
\hline 259 & P15151 & PVR & $\mathrm{NO}$ & NO & NO & iTRAQ \\
\hline 260 & O15212 & PFD6 & $\mathrm{NO}$ & NO & YES & iTRAQ \\
\hline 261 & P16870 & CBPE & $\mathrm{NO}$ & YES & NO & iTRAQ \\
\hline 262 & P50570 & DYN2 & $\mathrm{NO}$ & NO & YES & iTRAQ \\
\hline 263 & Q9NYQ8 & FAT2 & $\mathrm{NO}$ & NO & YES & iTRAQ \\
\hline 264 & O60687 & SRPX2 & $\mathrm{NO}$ & NO & NO & iTRAQ \\
\hline 265 & P49411 & EFTU & $\mathrm{NO}$ & NO & YES & iTRAQ \\
\hline 266 & P55001 & MFAP2 & $\mathrm{NO}$ & YES & YES & iTRAQ \\
\hline 267 & Q13217 & DNJC3 & $\mathrm{NO}$ & NO & NO & iTRAQ \\
\hline 268 & Q9NR99 & MXRA5 & $\mathrm{NO}$ & NO & NO & iTRAQ \\
\hline 269 & Е9PC48 & E9PC48 & $\mathrm{NO}$ & NO & YES & iTRAQ \\
\hline 270 & O43681 & ASNA & YES & NO & NO & iTRAQ \\
\hline 271 & P09758 & TACD2 & $\mathrm{NO}$ & YES & YES & iTRAQ \\
\hline 272 & P45880 & VDAC2 & $\mathrm{NO}$ & NO & YES & iTRAQ \\
\hline 273 & Q14563 & SEM3A & $\mathrm{NO}$ & $\mathrm{NO}$ & YES & iTRAQ \\
\hline 274 & Q9Y2T3 & GUAD & YES & NO & YES & iTRAQ \\
\hline
\end{tabular}




\begin{tabular}{|c|c|c|c|c|c|c|}
\hline 275 & Q00577 & PURA & $\mathrm{NO}$ & NO & YES & iTRAQ \\
\hline 276 & C9JNT3 & C9JNT3 & YES & NO & $\mathrm{NO}$ & iTRAQ \\
\hline 277 & P25685 & DNJB1 & YES & YES & $\mathrm{NO}$ & iTRAQ \\
\hline 278 & Q9P2B2 & FPRP & $\mathrm{NO}$ & YES & YES & iTRAQ \\
\hline 279 & P35556 & FBN2 & $\mathrm{NO}$ & NO & YES & iTRAQ \\
\hline 280 & P49748 & ACADV & $\mathrm{NO}$ & NO & NO & iTRAQ \\
\hline 281 & B1AHB1 & B1AHB1 & $\mathrm{NO}$ & NO & YES & iTRAQ \\
\hline 282 & C9JL12 & C9JL12 & YES & NO & $\mathrm{NO}$ & iTRAQ \\
\hline 283 & P18085 & ARF4 & $\mathrm{NO}$ & NO & $\mathrm{NO}$ & iTRAQ \\
\hline 284 & Q99650 & OSMR & $\mathrm{NO}$ & YES & YES & iTRAQ \\
\hline 285 & A2A274 & A2A274 & YES & NO & $\mathrm{NO}$ & iTRAQ \\
\hline 286 & Q86SQ4 & GP126 & $\mathrm{NO}$ & NO & $\mathrm{NO}$ & iTRAQ \\
\hline 287 & Q9UBQ6 & EXTL2 & YES & NO & YES & iTRAQ \\
\hline 288 & P09455 & RET1 & $\mathrm{NO}$ & NO & YES & iTRAQ \\
\hline 289 & P61764 & STXB1 & $\mathrm{NO}$ & NO & $\mathrm{NO}$ & iTRAQ \\
\hline 290 & Q9BZQ8 & NIBAN & YES & NO & $\mathrm{NO}$ & iTRAQ \\
\hline 291 & C9IZN5 & H7BYD0 & $\mathrm{NO}$ & NO & $\mathrm{NO}$ & iTRAQ \\
\hline 292 & B7Z214 & B7Z1C4 & YES & NO & $\mathrm{NO}$ & iTRAQ \\
\hline 293 & Q12805 & FBLN3 & YES & YES & $\mathrm{NO}$ & iTRAQ \\
\hline 294 & P62269 & RS18 & $\mathrm{NO}$ & NO & YES & iTRAQ \\
\hline 295 & P05198 & IF2A & $\mathrm{NO}$ & NO & YES & iTRAQ \\
\hline 296 & O15145 & ARPC3 & $\mathrm{NO}$ & NO & NO & iTRAQ \\
\hline 297 & P33908 & MA1A1 & YES & YES & YES & iTRAQ \\
\hline 298 & P30040 & ERP29 & $\mathrm{NO}$ & NO & YES & iTRAQ \\
\hline 299 & P49419 & AL7A1 & $\mathrm{NO}$ & NO & YES & iTRAQ \\
\hline 300 & C9JD73 & C9J177 & YES & YES & YES & iTRAQ \\
\hline 301 & P05156 & CFAI & $\mathrm{NO}$ & YES & $\mathrm{NO}$ & iTRAQ \\
\hline 302 & Q8WVM8 & SCFD1 & $\mathrm{NO}$ & NO & YES & iTRAQ \\
\hline 303 & A6NKH4 & A6NKH4 & YES & NO & $\mathrm{NO}$ & iTRAQ \\
\hline 304 & Q9UBG0 & MRC2 & YES & NO & $\mathrm{NO}$ & iTRAQ \\
\hline 305 & Р07858 & CATB & YES & NO & YES & iTRAQ \\
\hline 306 & Q92626 & PXDN & YES & NO & YES & iTRAQ \\
\hline 307 & B4E1N2 & B4E1N2 & YES & NO & YES & iTRAQ \\
\hline 308 & P24592 & IBP6 & YES & NO & $\mathrm{NO}$ & iTRAQ \\
\hline 309 & P12814 & ACTN1 & YES & NO & YES & iTRAQ \\
\hline 310 & P31431 & SDC4 & $\mathrm{NO}$ & NO & $\mathrm{NO}$ & iTRAQ \\
\hline 311 & P13647 & K2C5 & $\mathrm{NO}$ & NO & YES & iTRAQ \\
\hline 312 & Q9BWD1 & THIC & $\mathrm{NO}$ & NO & $\mathrm{NO}$ & iTRAQ \\
\hline 313 & O94907 & DKK1 & YES & YES & YES & iTRAQ \\
\hline 314 & Q9UBX1 & CATF & YES & NO & NO & iTRAQ \\
\hline 315 & P31153 & METK2 & YES & NO & YES & iTRAQ \\
\hline 316 & O60911 & CATL2 & $\mathrm{NO}$ & YES & YES & iTRAQ \\
\hline 317 & F5H013 & F5H013 & $\mathrm{NO}$ & NO & $\mathrm{NO}$ & iTRAQ \\
\hline 318 & P52292 & IMA2 & YES & YES & $\mathrm{NO}$ & iTRAQ \\
\hline 319 & Q14980 & NUMA1 & YES & YES & $\mathrm{NO}$ & iTRAQ \\
\hline 320 & O75531 & BAF & YES & NO & $\mathrm{NO}$ & iTRAQ \\
\hline 321 & Q14019 & COTL1 & NO & YES & YES & iTRAQ \\
\hline 322 & Q00688 & FKBP3 & $\mathrm{NO}$ & YES & YES & iTRAQ \\
\hline 323 & I3L4C9 & F5H6A3 & YES & YES & NO & iTRAQ \\
\hline 324 & Q9ULA0 & DNPEP & $\mathrm{NO}$ & NO & $\mathrm{NO}$ & iTRAQ \\
\hline 325 & A6NI00 & A6NI00 & $\mathrm{NO}$ & NO & $\mathrm{NO}$ & iTRAQ \\
\hline 326 & Q68BL7 & OLM2A & YES & YES & $\mathrm{NO}$ & iTRAQ \\
\hline 327 & В3КТC7 & B3КТC7 & $\mathrm{NO}$ & NO & $\mathrm{NO}$ & iTRAQ \\
\hline 328 & P50895 & BCAM & YES & NO & NO & iTRAQ \\
\hline 329 & P08107 & HSP71 & $\mathrm{NO}$ & NO & YES & iTRAQ \\
\hline 330 & F8WA83 & B5MCQ5 & $\mathrm{NO}$ & NO & YES & iTRAQ \\
\hline
\end{tabular}




\begin{tabular}{|c|c|c|c|c|c|c|}
\hline 331 & P30530 & UFO & YES & NO & NO & iTRAQ \\
\hline 332 & Q7KZF4 & SND1 & NO & NO & YES & iTRAQ \\
\hline 333 & D3DRA3 & D3DRA3 & YES & NO & YES & iTRAQ \\
\hline 334 & B4DGU4 & B4DGU4 & YES & NO & NO & iTRAQ \\
\hline 335 & Q15019 & SEPT2 & $\mathrm{NO}$ & YES & NO & iTRAQ \\
\hline 336 & G3V3X5 & G3V3X5 & $\mathrm{NO}$ & NO & YES & iTRAQ \\
\hline 337 & P26639 & SYTC & $\mathrm{NO}$ & NO & NO & iTRAQ \\
\hline 338 & P48723 & HSP13 & $\mathrm{NO}$ & NO & YES & iTRAQ \\
\hline 339 & O14980 & XPO1 & YES & NO & NO & iTRAQ \\
\hline 340 & O00764 & PDXK & $\mathrm{NO}$ & YES & YES & iTRAQ \\
\hline 341 & Q8WVQ1 & CANT1 & $\mathrm{NO}$ & YES & YES & iTRAQ \\
\hline 342 & E9PL22 & E9PL22 & NO & YES & YES & iTRAQ \\
\hline 343 & P55884 & EIF3B & $\mathrm{NO}$ & NO & NO & iTRAQ \\
\hline 344 & P36578 & RL4 & $\mathrm{NO}$ & NO & NO & iTRAQ \\
\hline 345 & P23246 & SFPQ & $\mathrm{NO}$ & NO & YES & iTRAQ \\
\hline 346 & D6RER5 & D6RER5 & YES & NO & NO & iTRAQ \\
\hline 347 & Q99460 & PSMD1 & $\mathrm{NO}$ & NO & NO & iTRAQ \\
\hline 348 & P53621 & COPA & $\mathrm{NO}$ & NO & YES & iTRAQ \\
\hline 349 & O43776 & SYNC & YES & NO & YES & iTRAQ \\
\hline 350 & P05556 & ITB1 & YES & NO & YES & iTRAQ \\
\hline 351 & Q14512 & FGFP1 & NO & NO & YES & iTRAQ \\
\hline 352 & Q92499 & DDX1 & $\mathrm{NO}$ & NO & NO & iTRAQ \\
\hline 353 & Q14574 & DSC3 & $\mathrm{NO}$ & NO & YES & iTRAQ \\
\hline 354 & B3KVR1 & B3KVR1 & $\mathrm{NO}$ & NO & YES & iTRAQ \\
\hline 355 & F8VV71 & F8VSD4 & $\mathrm{NO}$ & NO & NO & iTRAQ \\
\hline 356 & P61353 & RL27 & YES & YES & NO & iTRAQ \\
\hline 357 & D3YTI8 & D3YTB1 & YES & YES & YES & iTRAQ \\
\hline 358 & P22692 & IBP4 & $\mathrm{NO}$ & YES & NO & iTRAQ \\
\hline 359 & Q9UNM6 & PSD13 & YES & NO & YES & iTRAQ \\
\hline 360 & B5ME19 & B5ME19 & $\mathrm{NO}$ & YES & YES & iTRAQ \\
\hline 361 & Q15046 & SYK & $\mathrm{NO}$ & NO & NO & iTRAQ \\
\hline 362 & P10155 & RO60 & $\mathrm{NO}$ & NO & NO & iTRAQ \\
\hline 363 & Q6ZRP7 & QSOX2 & YES & NO & NO & iTRAQ \\
\hline 364 & O00622 & CYR61 & $\mathrm{NO}$ & YES & NO & iTRAQ \\
\hline 365 & O43747 & AP1G1 & $\mathrm{NO}$ & NO & YES & iTRAQ \\
\hline 366 & O43852 & CALU & $\mathrm{NO}$ & NO & YES & iTRAQ \\
\hline 367 & P60228 & EIF3E & $\mathrm{NO}$ & NO & YES & iTRAQ \\
\hline 368 & Q7Z6Z7 & HUWE1 & $\mathrm{NO}$ & NO & NO & iTRAQ \\
\hline 369 & O00231 & PSD11 & $\mathrm{NO}$ & NO & YES & iTRAQ \\
\hline 370 & Q08257 & QOR & $\mathrm{NO}$ & NO & NO & iTRAQ \\
\hline 371 & Q9BRK5 & CAB45 & YES & YES & YES & iTRAQ \\
\hline 372 & J3KQU0 & E9PKG6 & YES & NO & YES & iTRAQ \\
\hline 373 & A6NKB8 & A6NKB8 & $\mathrm{NO}$ & NO & NO & iTRAQ \\
\hline 374 & Q76M96 & CCD80 & YES & YES & NO & iTRAQ \\
\hline 375 & Q9H4M9 & EHD1 & $\mathrm{NO}$ & NO & NO & iTRAQ \\
\hline 376 & Q68CR9 & Q68CR9 & $\mathrm{NO}$ & NO & YES & iTRAQ \\
\hline 377 & P04843 & RPN1 & YES & NO & NO & iTRAQ \\
\hline 378 & Q9BPX3 & CND3 & $\mathrm{NO}$ & NO & YES & iTRAQ \\
\hline 379 & O15511 & ARPC5 & YES & NO & NO & iTRAQ \\
\hline 380 & Q9UQE7 & SMC3 & $\mathrm{NO}$ & YES & YES & iTRAQ \\
\hline 381 & P05997 & $\mathrm{CO} 5 \mathrm{~A} 2$ & YES & NO & NO & iTRAQ \\
\hline 382 & B4DM74 & B4DM74 & YES & NO & NO & iTRAQ \\
\hline 383 & Q8IYS5 & OSCAR & $\mathrm{NO}$ & YES & NO & iTRAQ \\
\hline 384 & Q9UN70 & PCDGK & $\mathrm{NO}$ & NO & YES & iTRAQ \\
\hline 385 & Q9UBT2 & SAE2 & $\mathrm{NO}$ & NO & YES & iTRAQ \\
\hline 386 & P27635 & RL10 & YES & NO & NO & iTRAQ \\
\hline
\end{tabular}




\begin{tabular}{|c|c|c|c|c|c|c|}
\hline 387 & O95433 & AHSA1 & $\mathrm{NO}$ & YES & YES & iTRAQ \\
\hline 388 & C9IZE4 & C9IZE4 & $\mathrm{NO}$ & NO & NO & iTRAQ \\
\hline 389 & A2A2D0 & $\mathrm{A} 2 \mathrm{~A} 2 \mathrm{D} 0$ & $\mathrm{NO}$ & NO & NO & iTRAQ \\
\hline 390 & B2RDM2 & B2RDM2 & $\mathrm{NO}$ & YES & NO & iTRAQ \\
\hline 391 & B4DLZ5 & B4DLZ5 & $\mathrm{NO}$ & YES & NO & iTRAQ \\
\hline 392 & Q9BXJ9 & NAA15 & $\mathrm{NO}$ & YES & NO & iTRAQ \\
\hline 393 & B4DKY1 & B4DKY1 & $\mathrm{NO}$ & NO & NO & iTRAQ \\
\hline 394 & Q7L576 & CYFP1 & $\mathrm{NO}$ & NO & NO & iTRAQ \\
\hline 395 & P50452 & SPB8 & YES & NO & YES & iTRAQ \\
\hline 396 & Q99584 & S10AD & YES & NO & YES & iTRAQ \\
\hline 397 & P41252 & SYIC & YES & NO & YES & iTRAQ \\
\hline 398 & P47755 & CAZA2 & $\mathrm{NO}$ & NO & NO & iTRAQ \\
\hline 399 & Q6P2Q9 & PRP8 & $\mathrm{NO}$ & NO & YES & iTRAQ \\
\hline 400 & B4DVY1 & B4DVY1 & $\mathrm{NO}$ & NO & NO & iTRAQ \\
\hline 401 & Q96CW1 & AP2M1 & $\mathrm{NO}$ & NO & NO & iTRAQ \\
\hline 402 & A6NFX8 & A6NFX8 & YES & NO & NO & iTRAQ \\
\hline 403 & A8K3Z3 & A8K3Z3 & $\mathrm{NO}$ & NO & NO & iTRAQ \\
\hline 404 & Q99536 & VAT1 & $\mathrm{NO}$ & YES & NO & iTRAQ \\
\hline 405 & B4DNJ6 & B4DNJ6 & $\mathrm{NO}$ & NO & YES & iTRAQ \\
\hline 406 & P14923 & PLAK & YES & NO & NO & iTRAQ \\
\hline 407 & P62854 & RS26 & YES & NO & YES & iTRAQ \\
\hline 408 & Q9BQA1 & MEP50 & $\mathrm{NO}$ & NO & YES & iTRAQ \\
\hline 409 & F5H7E2 & F5H7E2 & $\mathrm{NO}$ & YES & NO & iTRAQ \\
\hline 410 & Q92900 & RENT1 & YES & NO & NO & iTRAQ \\
\hline 411 & O76003 & GLRX3 & $\mathrm{NO}$ & NO & NO & iTRAQ \\
\hline 412 & B7Z815 & B7Z815 & $\mathrm{NO}$ & YES & NO & iTRAQ \\
\hline 413 & Q9UBB4 & ATX10 & YES & NO & NO & iTRAQ \\
\hline 414 & A6NJA2 & A6NJA2 & $\mathrm{NO}$ & NO & NO & iTRAQ \\
\hline 415 & P17858 & K6PL & $\mathrm{NO}$ & YES & NO & iTRAQ \\
\hline 416 & A0AVT1 & UBA6 & YES & NO & YES & iTRAQ \\
\hline 417 & P46109 & CRKL & $\mathrm{NO}$ & NO & YES & iTRAQ \\
\hline 418 & P37235 & HPCL1 & $\mathrm{NO}$ & YES & YES & iTRAQ \\
\hline 419 & B0QYN7 & B0QYN7 & $\mathrm{NO}$ & YES & YES & iTRAQ \\
\hline 420 & G3V2F7 & G3V2F7 & $\mathrm{NO}$ & NO & NO & iTRAQ \\
\hline 421 & F5H897 & F5H897 & $\mathrm{NO}$ & YES & NO & iTRAQ \\
\hline 422 & P78324 & SHPS1 & $\mathrm{NO}$ & YES & NO & iTRAQ \\
\hline 423 & Q8NBJ4 & GOLM1 & $\mathrm{NO}$ & NO & YES & iTRAQ \\
\hline 424 & P03950 & ANGI & $\mathrm{NO}$ & NO & YES & iTRAQ \\
\hline 425 & Q99471 & PFD5 & $\mathrm{NO}$ & NO & YES & iTRAQ \\
\hline 426 & P49767 & VEGFC & $\mathrm{NO}$ & NO & YES & iTRAQ \\
\hline 427 & A0AVL1 & A0AVL1 & $\mathrm{NO}$ & NO & YES & iTRAQ \\
\hline 428 & O00241 & SIRB1 & $\mathrm{NO}$ & NO & NO & iTRAQ \\
\hline 429 & O95881 & TXD12 & $\mathrm{NO}$ & NO & YES & iTRAQ \\
\hline 430 & B4E3K9 & B3KUK2 & $\mathrm{NO}$ & NO & NO & iTRAQ \\
\hline 431 & P43034 & LIS1 & $\mathrm{NO}$ & YES & NO & iTRAQ \\
\hline 432 & P63151 & $2 \mathrm{ABA}$ & $\mathrm{NO}$ & NO & NO & iTRAQ \\
\hline 433 & C9JNW5 & C9JNW5 & $\mathrm{NO}$ & NO & YES & iTRAQ \\
\hline 434 & Q8WUT4 & LRRN4 & $\mathrm{NO}$ & YES & NO & iTRAQ \\
\hline 435 & F5H124 & F5H6P7 & $\mathrm{NO}$ & NO & YES & iTRAQ \\
\hline 436 & P21583 & SCF & $\mathrm{NO}$ & YES & NO & iTRAQ \\
\hline 437 & Q9UBF2 & COPG2 & YES & NO & NO & iTRAQ \\
\hline 438 & Q9UGI8 & TES & $\mathrm{NO}$ & NO & NO & iTRAQ \\
\hline 439 & P35232 & PHB & $\mathrm{NO}$ & NO & NO & iTRAQ \\
\hline 440 & B2CPU0 & B2CPU0 & $\mathrm{NO}$ & NO & YES & iTRAQ \\
\hline 441 & Q9H3U1 & UN45A & $\mathrm{NO}$ & NO & NO & iTRAQ \\
\hline 442 & B0QY89 & B0QY89 & $\mathrm{NO}$ & YES & NO & iTRAQ \\
\hline
\end{tabular}




\begin{tabular}{|c|c|c|c|c|c|c|}
\hline 443 & Q9NPC4 & A4GAT & $\mathrm{NO}$ & YES & NO & iTRAQ \\
\hline 444 & Q5VYK3 & ECM29 & YES & YES & NO & iTRAQ \\
\hline 445 & P08621 & RU17 & YES & NO & YES & iTRAQ \\
\hline 446 & P51665 & PSD7 & $\mathrm{NO}$ & NO & NO & iTRAQ \\
\hline 447 & B3KRS5 & B3KRS5 & YES & NO & YES & iTRAQ \\
\hline 448 & Q13630 & FCL & $\mathrm{NO}$ & NO & NO & iTRAQ \\
\hline 449 & B5MC21 & B5MCZ3 & $\mathrm{NO}$ & NO & NO & iTRAQ \\
\hline 450 & Q9UKD2 & MRT4 & NO & YES & NO & iTRAQ \\
\hline 451 & P54920 & SNAA & $\mathrm{NO}$ & NO & NO & iTRAQ \\
\hline 452 & P21964 & COMT & NO & YES & YES & iTRAQ \\
\hline 453 & F5GYL8 & F5GYL8 & $\mathrm{NO}$ & NO & YES & iTRAQ \\
\hline 454 & B5ME97 & B5ME97 & NO & NO & NO & iTRAQ \\
\hline 455 & E5RJ48 & E5RGR0 & $\mathrm{NO}$ & YES & NO & iTRAQ \\
\hline 456 & Q13151 & ROA0 & $\mathrm{NO}$ & YES & NO & iTRAQ \\
\hline 457 & Р04844 & RPN2 & $\mathrm{NO}$ & NO & NO & iTRAQ \\
\hline 458 & F8W7S5 & F8W7S5 & $\mathrm{NO}$ & NO & YES & iTRAQ \\
\hline 459 & Q9P287 & BCCIP & $\mathrm{NO}$ & NO & NO & ITRAQ \\
\hline 460 & F5H4L7 & F5H4L 7 & $\mathrm{NO}$ & YES & NO & iTRAQ \\
\hline 461 & J3KNF6 & J3KNF6 & $\mathrm{NO}$ & NO & NO & iTRAQ \\
\hline 462 & B4DW05 & B4DP75 & $\mathrm{NO}$ & YES & NO & iTRAQ \\
\hline 463 & P36776 & LONM & YES & YES & NO & iTRAQ \\
\hline 464 & Q2TAY7 & SMU1 & NO & NO & YES & iTRAQ \\
\hline 465 & B7Z5W1 & B7Z5W1 & $\mathrm{NO}$ & NO & NO & iTRAQ \\
\hline 466 & Q9GZX9 & TWSG1 & $\mathrm{NO}$ & NO & NO & iTRAQ \\
\hline 467 & B3KVK7 & B3KVK7 & YES & NO & YES & ITRAQ \\
\hline 468 & H7C579 & H7C579 & $\mathrm{NO}$ & YES & $\mathrm{NO}$ & iTRAQ \\
\hline 469 & Q5JXH7 & Q5JXI8 & $\mathrm{NO}$ & YES & NO & iTRAQ \\
\hline 470 & D6RHU3 & D6RHU3 & $\mathrm{NO}$ & NO & NO & iTRAQ \\
\hline 471 & O75153 & CLU & YES & NO & NO & iTRAQ \\
\hline 472 & B3KS98 & B3KS98 & $\mathrm{NO}$ & NO & NO & iTRAQ \\
\hline 473 & P36542 & ATPG & $\mathrm{NO}$ & NO & NO & iTRAQ \\
\hline 474 & P31040 & DHSA & $\mathrm{NO}$ & NO & YES & iTRAQ \\
\hline 475 & P08670 & VIME & YES & NO & YES & iTRAQ \\
\hline 476 & B1AVU8 & B1AVU8,SAP & $\mathrm{NO}$ & YES & NO & iTRAQ \\
\hline 477 & Q15582 & BGH3 & $\mathrm{NO}$ & NO & NO & iTRAQ \\
\hline 478 & Q16787 & LAMA3 & $\mathrm{NO}$ & NO & YES & iTRAQ \\
\hline 479 & P16070 & CD44 & YES & NO & YES & iTRAQ \\
\hline 480 & P08727 & $\mathrm{K} 1 \mathrm{C} 19$ & YES & NO & YES & iTRAQ \\
\hline 481 & Q13421 & MSLN & $\mathrm{NO}$ & YES & YES & iTRAQ \\
\hline 482 & P15311 & EZRI & YES & NO & YES & iTRAQ \\
\hline 483 & B4DTG2 & B4DTG2 & YES & YES & YES & iTRAQ \\
\hline 484 & P47895 & AL1A3 & $\mathrm{NO}$ & YES & NO & iTRAQ \\
\hline 485 & P62158 & CALM & $\mathrm{NO}$ & NO & NO & iTRAQ \\
\hline 486 & P23526 & SAHH & $\mathrm{NO}$ & YES & YES & iTRAQ \\
\hline 487 & E9PJY4 & E9PLK3 & YES & YES & YES & iTRAQ \\
\hline 488 & Q9UNW1 & MINP1 & $\mathrm{NO}$ & NO & NO & iTRAQ \\
\hline 489 & Q15904 & VAS1 & $\mathrm{NO}$ & NO & YES & iTRAQ \\
\hline 490 & Q08629 & TICN1 & $\mathrm{NO}$ & NO & YES & iTRAQ \\
\hline 491 & Q08945 & SSRP1 & YES & NO & YES & iTRAQ \\
\hline 492 & P50502 & F10A1 & YES & NO & NO & iTRAQ \\
\hline 493 & P13284 & GILT & $\mathrm{NO}$ & YES & YES & iTRAQ \\
\hline 494 & P41250 & SYG & $\mathrm{NO}$ & NO & YES & iTRAQ \\
\hline 495 & F8W8J4 & F8W8J4 & $\mathrm{NO}$ & NO & YES & iTRAQ \\
\hline 496 & Q16695 & $\mathrm{H} 31 \mathrm{~T}$ & YES & NO & NO & iTRAQ \\
\hline 497 & P09874 & PARP1 & $\mathrm{NO}$ & YES & NO & iTRAQ \\
\hline 498 & H0YME6 & H7BYN4 & $\mathrm{NO}$ & NO & NO & iTRAQ \\
\hline
\end{tabular}




\begin{tabular}{|c|c|c|c|c|c|c|}
\hline 499 & B4DJP7 & B4DJP7 & $\mathrm{NO}$ & YES & NO & iTRAQ \\
\hline 500 & B3KSH1 & B3KSH1 & YES & YES & NO & iTRAQ \\
\hline 501 & P46821 & MAP1B & $\mathrm{NO}$ & YES & NO & iTRAQ \\
\hline 502 & E9PFT6 & E9PFT6 & YES & NO & NO & iTRAQ \\
\hline 503 & P09429 & HMGB1 & $\mathrm{NO}$ & YES & NO & iTRAQ \\
\hline 504 & Q32P28 & P3H1 & $\mathrm{NO}$ & NO & YES & iTRAQ \\
\hline 505 & Q9NZV1 & CRIM1 & $\mathrm{NO}$ & NO & NO & iTRAQ \\
\hline 506 & P29317 & EPHA2 & YES & NO & YES & iTRAQ \\
\hline 507 & O94925 & GLSK & YES & NO & YES & iTRAQ \\
\hline 508 & J3KR44 & F5GYJ8 & $\mathrm{NO}$ & NO & YES & iTRAQ \\
\hline 509 & Q96C86 & DCPS & YES & NO & NO & iTRAQ \\
\hline 510 & P35237 & SPB6 & YES & YES & NO & iTRAQ \\
\hline 511 & E7EUT8 & E7EUT8 & $\mathrm{NO}$ & NO & NO & iTRAQ \\
\hline 512 & P10412 & H14 & $\mathrm{NO}$ & NO & NO & iTRAQ \\
\hline 513 & Q9NSD9 & SYFB & $\mathrm{NO}$ & NO & NO & iTRAQ \\
\hline 514 & P10646 & TFPI1 & $\mathrm{NO}$ & NO & NO & iTRAQ \\
\hline 515 & Q15102 & PA1B3 & $\mathrm{NO}$ & NO & YES & iTRAQ \\
\hline 516 & J3QT22 & J3QT22 & $\mathrm{NO}$ & NO & YES & iTRAQ \\
\hline 517 & Q6PCB0 & VWA1 & $\mathrm{NO}$ & YES & NO & iTRAQ \\
\hline 518 & P15924 & DESP & $\mathrm{NO}$ & NO & YES & iTRAQ \\
\hline 519 & Q8WXF1 & PSPC1 & YES & NO & YES & iTRAQ \\
\hline 520 & Q63HN8 & RN213 & NO & NO & NO & iTRAQ \\
\hline 521 & F5H6E2 & F5H6E2 & YES & NO & NO & iTRAQ \\
\hline 522 & O14929 & HAT1 & $\mathrm{NO}$ & NO & NO & iTRAQ \\
\hline 523 & Q9Y5X9 & LIPE & $\mathrm{NO}$ & NO & NO & iTRAQ \\
\hline 524 & O43854 & EDIL3 & $\mathrm{NO}$ & NO & YES & iTRAQ \\
\hline 525 & P13861 & KAP2 & $\mathrm{NO}$ & NO & NO & iTRAQ \\
\hline 526 & Q14192 & FHL2 & YES & YES & NO & iTRAQ \\
\hline 527 & P22223 & CADH3 & NO & YES & NO & iTRAQ \\
\hline 528 & Q07954 & LRP1 & $\mathrm{NO}$ & NO & YES & iTRAQ \\
\hline 529 & E9PNS7 & E9PNS7 & YES & NO & YES & iTRAQ \\
\hline 530 & Q99714 & HCD2 & $\mathrm{NO}$ & YES & NO & iTRAQ \\
\hline 531 & P28340 & DPOD1 & $\mathrm{NO}$ & NO & YES & iTRAQ \\
\hline 532 & Q9NP79 & VTA1 & $\mathrm{NO}$ & NO & NO & iTRAQ \\
\hline 533 & J3KN01 & A8MQ02 & $\mathrm{NO}$ & NO & YES & iTRAQ \\
\hline 534 & P62304 & RUXE & YES & NO & NO & iTRAQ \\
\hline 535 & Q969H8 & CS010 & $\mathrm{NO}$ & YES & YES & iTRAQ \\
\hline 536 & P18754 & $\mathrm{RCC1}$ & $\mathrm{NO}$ & NO & YES & iTRAQ \\
\hline 537 & C9JGI3 & C9JGI3 & $\mathrm{NO}$ & YES & NO & iTRAQ \\
\hline 538 & E7EWT1 & E7EWT1 & $\mathrm{NO}$ & YES & NO & iTRAQ \\
\hline 539 & Q9BXR0 & TGT & $\mathrm{NO}$ & NO & NO & iTRAQ \\
\hline 540 & P38117 & ETFB & $\mathrm{NO}$ & YES & NO & iTRAQ \\
\hline 541 & E7EP74 & E7EP74 & $\mathrm{NO}$ & NO & NO & iTRAQ \\
\hline 542 & Q14008 & CKAP5 & $\mathrm{NO}$ & NO & NO & iTRAQ \\
\hline 543 & Q8IWX8 & CHERP & YES & NO & NO & iTRAQ \\
\hline 544 & P12109 & CO6A1 & $\mathrm{NO}$ & NO & NO & iTRAQ \\
\hline 545 & B4E3Q1 & B4E3Q1 & $\mathrm{NO}$ & NO & YES & iTRAQ \\
\hline 546 & Q5H9B5 & Q5H9A7 & YES & NO & NO & iTRAQ \\
\hline 547 & P07195 & LDHB & $\mathrm{NO}$ & NO & NO & iTRAQ \\
\hline 548 & Q00610 & CLH1 & $\mathrm{NO}$ & YES & YES & iTRAQ \\
\hline 549 & Q01082 & SPTB2 & $\mathrm{NO}$ & NO & YES & iTRAQ \\
\hline 550 & Q02818 & NUCB1 & $\mathrm{NO}$ & NO & NO & iTRAQ \\
\hline 551 & Q9UBP4 & DKK3 & $\mathrm{NO}$ & NO & YES & iTRAQ \\
\hline 552 & Q06481 & APLP2 & YES & NO & YES & iTRAQ \\
\hline 553 & B4DUR8 & B4DUR8 & $\mathrm{NO}$ & NO & YES & iTRAQ \\
\hline 554 & Q14974 & IMB1 & $\mathrm{NO}$ & NO & NO & iTRAQ \\
\hline
\end{tabular}




\begin{tabular}{|c|c|c|c|c|c|c|}
\hline 555 & G5EA52 & G5EA52 & $\mathrm{NO}$ & YES & YES & iTRAQ \\
\hline 556 & B4DTU0 & B4DTU0 & $\mathrm{NO}$ & NO & NO & iTRAQ \\
\hline 557 & P55268 & LAMB2 & $\mathrm{NO}$ & NO & NO & iTRAQ \\
\hline 558 & P12110 & CO6A2 & $\mathrm{NO}$ & NO & YES & iTRAQ \\
\hline 559 & Q08211 & DHX9 & $\mathrm{NO}$ & NO & YES & iTRAQ \\
\hline 560 & Q01813 & К6РP & YES & NO & YES & iTRAQ \\
\hline 561 & P19022 & CADH2 & $\mathrm{NO}$ & NO & NO & iTRAQ \\
\hline 562 & Q9Y240 & CLC11 & $\mathrm{NO}$ & NO & YES & iTRAQ \\
\hline 563 & P50454 & SERPH & $\mathrm{NO}$ & NO & NO & iTRAQ \\
\hline 564 & Q86VP6 & CAND1 & $\mathrm{NO}$ & NO & YES & iTRAQ \\
\hline 565 & O75326 & SEM7A & $\mathrm{NO}$ & NO & YES & iTRAQ \\
\hline 566 & O00299 & CLIC1 & $\mathrm{NO}$ & NO & NO & iTRAQ \\
\hline 567 & P78527 & PRKDC & YES & NO & YES & iTRAQ \\
\hline 568 & P30153 & $2 \mathrm{AAA}$ & $\mathrm{NO}$ & NO & YES & iTRAQ \\
\hline 569 & Q12905 & ILF2 & YES & YES & YES & iTRAQ \\
\hline 570 & Q16531 & DDB1 & $\mathrm{NO}$ & NO & NO & iTRAQ \\
\hline 571 & H0Y8E6 & H0Y8E6 & $\mathrm{NO}$ & YES & NO & iTRAQ \\
\hline 572 & B7ZKN0 & B7ZKN0 & $\mathrm{NO}$ & NO & NO & iTRAQ \\
\hline 573 & Q9Y265 & RUVB1 & $\mathrm{NO}$ & NO & NO & iTRAQ \\
\hline 574 & P62701 & RS4X & $\mathrm{NO}$ & NO & NO & iTRAQ \\
\hline 575 & P15018 & LIF & YES & YES & YES & iTRAQ \\
\hline 576 & P19021 & AMD & NO & NO & NO & iTRAQ \\
\hline 577 & O95407 & TNF6B & YES & NO & YES & iTRAQ \\
\hline 578 & P20700 & LMNB1 & YES & NO & NO & iTRAQ \\
\hline 579 & O15144 & ARPC2 & $\mathrm{NO}$ & NO & NO & iTRAQ \\
\hline 580 & P35555 & FBN1 & $\mathrm{NO}$ & NO & YES & iTRAQ \\
\hline 581 & Q15393 & SF3B3 & YES & NO & YES & iTRAQ \\
\hline 582 & Q14152 & EIF3A & $\mathrm{NO}$ & YES & NO & iTRAQ \\
\hline 583 & P62241 & RS8 & $\mathrm{NO}$ & NO & NO & iTRAQ \\
\hline 584 & Q14126 & DSG2 & $\mathrm{NO}$ & NO & YES & iTRAQ \\
\hline 585 & J3KNP4 & J3KNP4 & $\mathrm{NO}$ & NO & YES & iTRAQ \\
\hline 586 & D6RBV2 & D6RBV2 & $\mathrm{NO}$ & NO & NO & iTRAQ \\
\hline 587 & P39023 & RL3 & YES & NO & NO & iTRAQ \\
\hline 588 & Q92743 & HTRA1 & YES & YES & NO & iTRAQ \\
\hline 589 & P51149 & RAB7A & $\mathrm{NO}$ & NO & YES & iTRAQ \\
\hline 590 & P52565 & GDIR1 & YES & NO & YES & iTRAQ \\
\hline 591 & P50914 & RL14 & $\mathrm{NO}$ & NO & YES & iTRAQ \\
\hline 592 & P10124 & SRGN & $\mathrm{NO}$ & NO & YES & iTRAQ \\
\hline 593 & P06703 & S10A6 & $\mathrm{NO}$ & NO & NO & iTRAQ \\
\hline 594 & P13497 & BMP1 & $\mathrm{NO}$ & YES & YES & iTRAQ \\
\hline 595 & P10253 & LYAG & $\mathrm{NO}$ & NO & YES & iTRAQ \\
\hline 596 & P61604 & CH10 & $\mathrm{NO}$ & NO & YES & iTRAQ \\
\hline 597 & Q9Y230 & RUVB2 & $\mathrm{NO}$ & YES & YES & iTRAQ \\
\hline 598 & O00571 & DDX3X & $\mathrm{NO}$ & NO & NO & iTRAQ \\
\hline 599 & O43143 & DHX15 & $\mathrm{NO}$ & NO & YES & iTRAQ \\
\hline 600 & Q96QK1 & VPS35 & $\mathrm{NO}$ & NO & NO & iTRAQ \\
\hline 601 & Q15181 & IPYR & YES & NO & YES & iTRAQ \\
\hline 602 & Q96TA1 & NIBL1 & $\mathrm{NO}$ & NO & YES & iTRAQ \\
\hline 603 & Q9P258 & RCC2 & $\mathrm{NO}$ & NO & YES & iTRAQ \\
\hline 604 & P47897 & SYQ & $\mathrm{NO}$ & NO & NO & iTRAQ \\
\hline 605 & Q06323 & PSME1 & $\mathrm{NO}$ & NO & YES & iTRAQ \\
\hline 606 & Q14566 & MCM6 & YES & NO & YES & iTRAQ \\
\hline 607 & Q2VIR3 & IF2GL & $\mathrm{NO}$ & NO & NO & iTRAQ \\
\hline 608 & P12270 & TPR & $\mathrm{NO}$ & NO & NO & iTRAQ \\
\hline 609 & P35221 & CTNA1 & $\mathrm{NO}$ & NO & NO & iTRAQ \\
\hline 610 & Q96P70 & IPO9 & YES & NO & YES & iTRAQ \\
\hline
\end{tabular}




\begin{tabular}{|c|c|c|c|c|c|c|}
\hline 611 & P30405 & PPIF & $\mathrm{NO}$ & YES & NO & iTRAQ \\
\hline 612 & P00505 & AATM & $\mathrm{NO}$ & YES & NO & iTRAQ \\
\hline 613 & P07814 & SYEP & $\mathrm{NO}$ & NO & YES & iTRAQ \\
\hline 614 & Q9H0U4 & RAB1B & $\mathrm{NO}$ & NO & YES & iTRAQ \\
\hline 615 & C9JZR2 & C9JZR2 & $\mathrm{NO}$ & NO & YES & iTRAQ \\
\hline 616 & P33993 & MCM7 & $\mathrm{NO}$ & NO & NO & iTRAQ \\
\hline 617 & P22234 & PUR6 & YES & NO & NO & iTRAQ \\
\hline 618 & P46778 & RL21 & YES & YES & YES & iTRAQ \\
\hline 619 & Q92896 & GSLG1 & $\mathrm{NO}$ & YES & NO & iTRAQ \\
\hline 620 & J3KPV4 & J3KPV4 & $\mathrm{NO}$ & NO & YES & iTRAQ \\
\hline 621 & P67775 & PP2AA & $\mathrm{NO}$ & NO & NO & iTRAQ \\
\hline 622 & F8W181 & F8W181 & $\mathrm{NO}$ & NO & NO & iTRAQ \\
\hline 623 & Q6P2E9 & EDC4 & $\mathrm{NO}$ & YES & NO & iTRAQ \\
\hline 624 & O94826 & TOM70 & YES & NO & NO & iTRAQ \\
\hline 625 & Q99622 & $\mathrm{C} 10$ & NO & NO & YES & iTRAQ \\
\hline 626 & B1AKP7 & B1AKP7 & $\mathrm{NO}$ & YES & NO & iTRAQ \\
\hline 627 & Q6ZR08 & DYH12 & $\mathrm{NO}$ & YES & NO & iTRAQ \\
\hline 628 & E9PJD9 & E9PLL6 & YES & NO & NO & iTRAQ \\
\hline 629 & O00232 & PSD12 & NO & YES & NO & iTRAQ \\
\hline 630 & Q6WCQ1 & MPRIP & $\mathrm{NO}$ & NO & YES & iTRAQ \\
\hline 631 & Q9BTE3 & МCMBP & $\mathrm{NO}$ & NO & NO & iTRAQ \\
\hline 632 & Q13610 & PWP1 & $\mathrm{NO}$ & NO & NO & iTRAQ \\
\hline 633 & Q9BQ52 & RNZ2 & $\mathrm{NO}$ & YES & NO & iTRAQ \\
\hline 634 & P26196 & DDX6 & $\mathrm{NO}$ & NO & NO & iTRAQ \\
\hline 635 & Q14738 & $2 \mathrm{~A} 5 \mathrm{D}$ & $\mathrm{NO}$ & NO & NO & iTRAQ \\
\hline 636 & F6XY72 & F6XY72 & $\mathrm{NO}$ & NO & NO & iTRAQ \\
\hline 637 & P63000 & RAC1 & $\mathrm{NO}$ & YES & NO & iTRAQ \\
\hline 638 & Q9UKV8 & $\mathrm{AGO} 2$ & $\mathrm{NO}$ & NO & NO & iTRAQ \\
\hline 639 & O95347 & SMC2 & $\mathrm{NO}$ & NO & NO & iTRAQ \\
\hline 640 & Q15021 & CND1 & YES & NO & NO & iTRAQ \\
\hline 641 & Q86TU7 & SETD3 & YES & NO & NO & iTRAQ \\
\hline 642 & B0UX83 & B0UX83 & $\mathrm{NO}$ & NO & NO & iTRAQ \\
\hline 643 & O43396 & TXNL1 & $\mathrm{NO}$ & NO & NO & iTRAQ \\
\hline 644 & Q9H488 & OFUT1 & $\mathrm{NO}$ & NO & YES & iTRAQ \\
\hline 645 & Q03154 & ACY1 & $\mathrm{NO}$ & NO & NO & iTRAQ \\
\hline 646 & B4DFK6 & B4DFK6 & $\mathrm{NO}$ & NO & YES & iTRAQ \\
\hline 647 & P52735 & VAV2 & $\mathrm{NO}$ & YES & NO & iTRAQ \\
\hline 648 & P62330 & ARF6 & $\mathrm{NO}$ & NO & YES & iTRAQ \\
\hline 649 & O14737 & PDCD5 & $\mathrm{NO}$ & YES & YES & iTRAQ \\
\hline 650 & Q8NE71 & ABCF 1 & YES & YES & NO & iTRAQ \\
\hline 651 & O14578 & CTRO & $\mathrm{NO}$ & NO & NO & iTRAQ \\
\hline 652 & B4DW92 & B4DW92 & YES & NO & NO & iTRAQ \\
\hline 653 & P07384 & CAN1 & YES & YES & NO & iTRAQ \\
\hline 654 & P08123 & CO1A2 & $\mathrm{NO}$ & YES & YES & iTRAQ \\
\hline 655 & Q13641 & TPBG & $\mathrm{NO}$ & NO & YES & iTRAQ \\
\hline 656 & A8MU58 & A8MU58 & $\mathrm{NO}$ & NO & NO & iTRAQ \\
\hline 657 & Q2NL82 & TSR1 & $\mathrm{NO}$ & YES & NO & iTRAQ \\
\hline 658 & Q09161 & NCBP1 & YES & NO & NO & iTRAQ \\
\hline 659 & O14497 & ARI1A & NO & NO & NO & iTRAQ \\
\hline 660 & C9JPG5 & C9JPG5 & $\mathrm{NO}$ & NO & NO & iTRAQ \\
\hline 661 & P49366 & DHYS & $\mathrm{NO}$ & NO & NO & iTRAQ \\
\hline 662 & P41567 & EIF1 & $\mathrm{NO}$ & YES & NO & iTRAQ \\
\hline 663 & C9JG87 & C9JG87 & $\mathrm{NO}$ & YES & NO & iTRAQ \\
\hline 664 & С9J4M6 & C9J2Y9 & $\mathrm{NO}$ & NO & NO & iTRAQ \\
\hline 665 & H0YLH3 & H0YLH3 & $\mathrm{NO}$ & NO & NO & iTRAQ \\
\hline 666 & Q15056 & IF4H & $\mathrm{NO}$ & NO & NO & iTRAQ \\
\hline
\end{tabular}




\begin{tabular}{|c|c|c|c|c|c|c|}
\hline 667 & P54886 & P5CS & $\mathrm{NO}$ & YES & YES & iTRAQ \\
\hline 668 & P19875 & CXCL2 & $\mathrm{NO}$ & YES & $\mathrm{NO}$ & iTRAQ \\
\hline 669 & Q9NQW6 & ANLN & $\mathrm{NO}$ & NO & $\mathrm{NO}$ & iTRAQ \\
\hline 670 & E7EW00 & E7ET15 & $\mathrm{NO}$ & NO & $\mathrm{NO}$ & iTRAQ \\
\hline 671 & B4DVN3 & B4DQT1 & $\mathrm{NO}$ & NO & $\mathrm{NO}$ & iTRAQ \\
\hline 672 & E9PF63 & E9PF63 & $\mathrm{NO}$ & NO & $\mathrm{NO}$ & iTRAQ \\
\hline 673 & O76061 & STC2 & $\mathrm{NO}$ & NO & $\mathrm{NO}$ & iTRAQ \\
\hline 674 & F8VPD4 & F8VPD4 & YES & NO & $\mathrm{NO}$ & iTRAQ \\
\hline 675 & H0Y6E7 & H0Y6E7 & $\mathrm{NO}$ & NO & $\mathrm{NO}$ & iTRAQ \\
\hline 676 & B4DGP8 & B4DGP8 & YES & YES & $\mathrm{NO}$ & iTRAQ \\
\hline 677 & F5H1A8 & F5H1A8 & $\mathrm{NO}$ & NO & $\mathrm{NO}$ & iTRAQ \\
\hline 678 & P27695 & APEX1 & $\mathrm{NO}$ & NO & $\mathrm{NO}$ & iTRAQ \\
\hline 679 & Q9Y376 & CAB39 & $\mathrm{NO}$ & YES & $\mathrm{NO}$ & iTRAQ \\
\hline 680 & P35442 & TSP2 & $\mathrm{NO}$ & YES & YES & iTRAQ \\
\hline 681 & P11766 & ADHX & $\mathrm{NO}$ & NO & YES & iTRAQ \\
\hline 682 & Q8N163 & K1967 & $\mathrm{NO}$ & NO & YES & iTRAQ \\
\hline 683 & P09661 & RU2A & $\mathrm{NO}$ & NO & $\mathrm{NO}$ & iTRAQ \\
\hline 684 & P55809 & SCOT1 & $\mathrm{NO}$ & YES & $\mathrm{NO}$ & iTRAQ \\
\hline 685 & Q99873 & ANM1 & $\mathrm{NO}$ & NO & YES & iTRAQ \\
\hline 686 & Q06124 & PTN11 & $\mathrm{NO}$ & NO & $\mathrm{NO}$ & iTRAQ \\
\hline 687 & P78347 & GTF2I & $\mathrm{NO}$ & NO & $\mathrm{NO}$ & iTRAQ \\
\hline 688 & P04080 & CYTB & $\mathrm{NO}$ & NO & $\mathrm{NO}$ & iTRAQ \\
\hline 689 & P05141 & ADT2 & $\mathrm{NO}$ & NO & YES & iTRAQ \\
\hline 690 & P27694 & RFA1 & $\mathrm{NO}$ & NO & YES & iTRAQ \\
\hline 691 & B4E2J3 & B4E2J3 & $\mathrm{NO}$ & NO & $\mathrm{NO}$ & iTRAQ \\
\hline 692 & P61313 & RL15 & YES & NO & $\mathrm{NO}$ & iTRAQ \\
\hline 693 & E7ESE0 & D6RAN4 & YES & NO & YES & iTRAQ \\
\hline 694 & Q9BVP2 & GNL3 & $\mathrm{NO}$ & YES & $\mathrm{NO}$ & iTRAQ \\
\hline 695 & Q9BZK7 & TBL1R & $\mathrm{NO}$ & NO & $\mathrm{NO}$ & iTRAQ \\
\hline 696 & A8MW61 & A8MW61 & $\mathrm{NO}$ & YES & $\mathrm{NO}$ & iTRAQ \\
\hline 697 & P68104 & EF1A1 & $\mathrm{NO}$ & NO & $\mathrm{NO}$ & iTRAQ \\
\hline 698 & Q12841 & FSTL1 & $\mathrm{NO}$ & NO & YES & iTRAQ \\
\hline 699 & P34932 & HSP74 & $\mathrm{NO}$ & NO & YES & iTRAQ \\
\hline 700 & P26022 & PTX3 & $\mathrm{NO}$ & NO & YES & iTRAQ \\
\hline 701 & F5H025 & F5H025 & $\mathrm{NO}$ & NO & YES & iTRAQ \\
\hline 702 & I3L404 & H0YEN5 & $\mathrm{NO}$ & NO & $\mathrm{NO}$ & iTRAQ \\
\hline 703 & D6RAT0 & D6RAT0 & $\mathrm{NO}$ & YES & $\mathrm{NO}$ & iTRAQ \\
\hline 704 & B7ZL00 & B7ZL00 & $\mathrm{NO}$ & YES & $\mathrm{NO}$ & iTRAQ \\
\hline 705 & A6NG06 & A6NG06 & YES & NO & $\mathrm{NO}$ & iTRAQ \\
\hline 706 & Q92520 & FAM3C & $\mathrm{NO}$ & YES & $\mathrm{NO}$ & iTRAQ \\
\hline 707 & P46781 & RS9 & $\mathrm{NO}$ & $\mathrm{NO}$ & YES & iTRAQ \\
\hline 708 & Q5VY30 & Q5VY30 & YES & NO & YES & iTRAQ \\
\hline 709 & P21589 & $5 \mathrm{NTD}$ & $\mathrm{NO}$ & YES & $\mathrm{NO}$ & iTRAQ \\
\hline 710 & Q13444 & ADA15 & YES & NO & $\mathrm{NO}$ & iTRAQ \\
\hline 711 & A6NKQ9 & CGB1 & YES & NO & $\mathrm{NO}$ & iTRAQ \\
\hline 712 & P55036 & PSMD4 & $\mathrm{NO}$ & NO & $\mathrm{NO}$ & iTRAQ \\
\hline 713 & A8MXP8 & F8WCY5 & $\mathrm{NO}$ & NO & $\mathrm{NO}$ & iTRAQ \\
\hline 714 & P48147 & PPCE & $\mathrm{NO}$ & NO & $\mathrm{NO}$ & iTRAQ \\
\hline 715 & Q9NS15 & LTBP3 & $\mathrm{NO}$ & YES & $\mathrm{NO}$ & iTRAQ \\
\hline 716 & G3V531 & F5H365 & $\mathrm{NO}$ & NO & $\mathrm{NO}$ & iTRAQ \\
\hline 717 & E9PF18 & E9PF18 & $\mathrm{NO}$ & NO & $\mathrm{NO}$ & iTRAQ \\
\hline 718 & G3V126 & G3V126 & $\mathrm{NO}$ & NO & $\mathrm{NO}$ & iTRAQ \\
\hline 719 & B4E2W0 & B4E2W0 & YES & NO & $\mathrm{NO}$ & iTRAQ \\
\hline 720 & P48729 & $\mathrm{KC} 1 \mathrm{~A}$ & $\mathrm{NO}$ & NO & $\mathrm{NO}$ & iTRAQ \\
\hline 721 & D6RBW1 & D6RBW1 & $\mathrm{NO}$ & NO & YES & iTRAQ \\
\hline 722 & B4DE16 & B4DE16 & $\mathrm{NO}$ & YES & $\mathrm{NO}$ & iTRAQ \\
\hline
\end{tabular}


723 H7C215 H7C0V4

$\mathrm{NO}$

YES

NO

iTRAQ

724 Q8TEQ6

GEMI5

$\mathrm{NO}$

NO

$\mathrm{NO}$

ITRAQ 
Table S7. Information of overlapped proteins identified by both 2DE and iTRAQ.

\begin{tabular}{|c|c|c|c|}
\hline NO. & $\begin{array}{l}\text { Swissprot } \\
\text { number }\end{array}$ & $\begin{array}{l}\text { YES or NO different by } 2 \mathrm{DE} \text { in } 5637 \\
\text { vs. SV-HUC-1 or T24 vs. SV-HUC-1 }\end{array}$ & $\begin{array}{l}\text { YES or NO same significantly abundance } \\
\text { change pattern between 2DE and iTRAQ }\end{array}$ \\
\hline 1 & P31947 & YES & YES \\
\hline 2 & O95336 & YES & YES \\
\hline 3 & P04075 & YES & YES \\
\hline 4 & P15121 & YES & YES \\
\hline 5 & P61769 & YES & YES \\
\hline 6 & P04792 & YES & YES \\
\hline 7 & Q92876 & YES & YES \\
\hline 8 & P08253 & YES & YES \\
\hline 9 & P61970 & YES & YES \\
\hline 10 & P09486 & YES & YES \\
\hline 11 & P36952 & YES & YES \\
\hline 12 & Q9GZM7 & YES & YES \\
\hline 13 & P07996 & YES & YES \\
\hline 14 & P31947 & YES & YES \\
\hline 15 & O95336 & YES & YES \\
\hline 16 & P15121 & YES & YES \\
\hline 17 & P62805 & YES & YES \\
\hline 18 & P46087 & YES & YES \\
\hline 19 & P49720 & YES & YES \\
\hline 20 & P36952 & YES & YES \\
\hline 21 & P05067 & YES & YES \\
\hline 22 & O00468 & YES & YES \\
\hline 23 & P01034 & YES & YES \\
\hline 24 & Р04406 & YES & YES \\
\hline 25 & P09382 & YES & YES \\
\hline 26 & P05121 & YES & YES \\
\hline 27 & P16035 & YES & YES \\
\hline 28 & P02751 & YES & NO \\
\hline 29 & P02768 & YES & $\mathrm{NO}$ \\
\hline 30 & P46087 & YES & $\mathrm{NO}$ \\
\hline 31 & P06748 & YES & $\mathrm{NO}$ \\
\hline 32 & P07339 & YES & $\mathrm{NO}$ \\
\hline 33 & P14174 & YES & $\mathrm{NO}$ \\
\hline 34 & P00558 & YES & $\mathrm{NO}$ \\
\hline 35 & P07737 & YES & $\mathrm{NO}$ \\
\hline 36 & P22626 & YES & $\mathrm{NO}$ \\
\hline 37 & P60174 & YES & $\mathrm{NO}$ \\
\hline 38 & P04792 & YES & $\mathrm{NO}$ \\
\hline 39 & P25787 & $\mathrm{NO}$ & $\mathrm{NO}$ \\
\hline 40 & P09486 & YES & $\mathrm{NO}$ \\
\hline 41 & P09211 & $\mathrm{NO}$ & $\mathrm{NO}$ \\
\hline 42 & P06733 & $\mathrm{NO}$ & $\mathrm{NO}$ \\
\hline 43 & P18669 & YES & $\mathrm{NO}$ \\
\hline 44 & P39687 & YES & $\mathrm{NO}$ \\
\hline 45 & P10809 & YES & $\mathrm{NO}$ \\
\hline 46 & P23528 & YES & $\mathrm{NO}$ \\
\hline 47 & P16930 & YES & $\mathrm{NO}$ \\
\hline 48 & Q13283 & YES & $\mathrm{NO}$ \\
\hline 49 & P62805 & YES & $\mathrm{NO}$ \\
\hline 50 & P14618 & YES & $\mathrm{NO}$ \\
\hline 51 & P11047 & YES & $\mathrm{NO}$ \\
\hline
\end{tabular}




\begin{tabular}{llll}
52 & $\mathrm{P} 40926$ & YES & NO \\
53 & $\mathrm{Q} 99497$ & YES & NO \\
54 & $\mathrm{P} 12004$ & YES & NO \\
55 & $\mathrm{P} 30086$ & YES & NO \\
56 & $\mathrm{P} 98160$ & YES & NO \\
57 & $\mathrm{P} 32119$ & YES & NO \\
58 & $\mathrm{P} 30041$ & YES & NO \\
59 & $\mathrm{P} 28066$ & YES & NO \\
60 & $\mathrm{P} 49721$ & YES & NO \\
61 & $\mathrm{P} 49720$ & YES & NO \\
62 & $\mathrm{P} 28070$ & YES & NO \\
63 & $\mathrm{P} 28074$ & YES & NO \\
64 & $\mathrm{P} 28072$ & YES & NO \\
65 & $\mathrm{Q} 99436$ & YES & NO \\
66 & $\mathrm{Q} 15223$ & YES & NO \\
67 & $\mathrm{Q} 01105$ & YES & NO \\
68 & $\mathrm{P} 00441$ & YES & NO \\
69 & $\mathrm{Q} 04837$ & YES & NO \\
70 & $\mathrm{P} 04040$ & NO & YES \\
71 & $\mathrm{P} 61970$ & YES & NO \\
72 & $\mathrm{P} 37802$ & NO & NO \\
73 & $\mathrm{P} 07237$ & NO & \\
\hline
\end{tabular}

(NO.1-NO.13 represented the 13 proteins showed same significantly abundance change pattern between 2DE and iTRAQ in 5637 vs. SV-HUC-1. NO.14-NO.27 represented the 14 proteins showed same significantly abundance change pattern between 2DE and iTRAQ in T24 vs. SV-HUC-1. NO.28-NO.73 stood for the 46 proteins that showed conflict abundance change between 2DE and iTRAQ.) 
Table S8. Identification information of 427 proteins detected in urine samples by SWATH.

\begin{tabular}{|c|c|c|c|c|c|}
\hline NO. & $\begin{array}{c}\text { Swissprot } \\
\text { number }\end{array}$ & $\begin{array}{l}\text { Protein } \\
\text { name }\end{array}$ & $\begin{array}{l}\text { overlapped with } \\
\text { the } 724 \text { proteins }\end{array}$ & p-value & $\begin{array}{c}\text { BCa vs. Normal } \\
\text { fold changes }\end{array}$ \\
\hline 1 & O00468 & AGRIN & YES & 0.0000 & 0.57 \\
\hline 2 & O00560 & SDCB1 & YES & 0.0000 & 0.37 \\
\hline 3 & O14980 & XPO1 & YES & 0.8879 & 1.07 \\
\hline 4 & O75369 & FLNB & YES & 0.0012 & 0.48 \\
\hline 5 & O95336 & 6PGL & YES & 0.0170 & 0.66 \\
\hline 6 & P00338 & LDHA & YES & 0.0038 & 0.57 \\
\hline 7 & P00441 & SODC & YES & 0.0000 & 0.38 \\
\hline 8 & P00558 & PGK1 & YES & 0.3672 & 1.22 \\
\hline 9 & P00736 & C1R & YES & 0.0000 & 3.54 \\
\hline 10 & P00966 & ASSY & YES & 0.0000 & 0.21 \\
\hline 11 & P01008 & ANT3 & YES & 0.0000 & 1.98 \\
\hline 12 & P01009 & A1AT & YES & 0.0000 & 1.84 \\
\hline 13 & P01024 & $\mathrm{CO} 3$ & YES & 0.0000 & 7.83 \\
\hline 14 & P01033 & TIMP1 & YES & 0.0016 & 1.35 \\
\hline 15 & P01034 & CYTC & YES & 0.0007 & 0.64 \\
\hline 16 & P02649 & APOE & YES & 0.0000 & 1.71 \\
\hline 17 & P02751 & FINC & YES & 0.0000 & 1.28 \\
\hline 18 & P02768 & ALBU & YES & 0.0000 & 2.43 \\
\hline 19 & P04066 & FUCO & YES & 0.0000 & 0.46 \\
\hline 20 & P04075 & ALDOA & YES & 0.0041 & 2.22 \\
\hline 21 & P04080 & СYТВ & YES & 0.4282 & 1.15 \\
\hline 22 & P04083 & ANXA1 & YES & 0.0001 & 1.43 \\
\hline 23 & P04264 & $\mathrm{K} 2 \mathrm{C} 1$ & YES & 0.0000 & 1.91 \\
\hline 24 & P04406 & G3P & YES & 0.0876 & 0.74 \\
\hline 25 & P04792 & HSPB1 & YES & 0.0054 & 0.64 \\
\hline 26 & P05067 & A4 & YES & 0.1862 & 0.79 \\
\hline 27 & P05156 & CFAI & YES & 0.0049 & 1.20 \\
\hline 28 & P06703 & S10A6 & YES & 0.1126 & 0.48 \\
\hline 29 & P06733 & ENOA & YES & 0.0052 & 1.74 \\
\hline 30 & P07195 & LDHB & YES & 0.0000 & 0.54 \\
\hline 31 & P07237 & PDIA1 & YES & 0.0335 & 0.61 \\
\hline 32 & P07339 & CATD & YES & 0.0000 & 0.63 \\
\hline 33 & P07737 & PROF1 & YES & 0.0004 & 1.86 \\
\hline 34 & P07858 & CATB & YES & 0.0000 & 0.58 \\
\hline 35 & Р07996 & TSP1 & YES & 0.0000 & 0.55 \\
\hline 36 & P08107 & HS71A & YES & 0.2466 & 0.87 \\
\hline 37 & P08123 & $\mathrm{CO} 1 \mathrm{~A} 2$ & YES & 0.0591 & 0.67 \\
\hline 38 & P09211 & GSTP1 & YES & 0.0288 & 1.19 \\
\hline 39 & P09668 & CATH & YES & 0.0001 & 0.58 \\
\hline 40 & P10253 & LYAG & YES & 0.0000 & 0.39 \\
\hline 41 & P11047 & LAMC1 & YES & 0.0002 & 0.62 \\
\hline 42 & P11717 & MPRI & YES & 0.0000 & 1.34 \\
\hline 43 & P12109 & CO6A1 & YES & 0.0000 & 0.72 \\
\hline 44 & P12270 & TPR & YES & 0.0000 & 2.22 \\
\hline 45 & P12277 & KCRB & YES & 0.0000 & 0.28 \\
\hline 46 & P12830 & CADH1 & YES & 0.0000 & 0.54 \\
\hline 47 & P13284 & GILT & YES & 0.0000 & 0.26 \\
\hline 48 & P13796 & PLSL & YES & 0.0000 & 3.66 \\
\hline
\end{tabular}




\begin{tabular}{|c|c|c|c|c|c|}
\hline 49 & P14543 & NID1 & YES & 0.0846 & 0.64 \\
\hline 50 & P14618 & KPYM & YES & 0.1862 & 0.75 \\
\hline 51 & P15151 & PVR & YES & 0.9876 & 1.01 \\
\hline 52 & P15311 & EZRI & YES & 0.1330 & 0.77 \\
\hline 53 & P16035 & TIMP2 & YES & 0.0006 & 0.65 \\
\hline 54 & P16070 & CD44 & YES & 0.0000 & 0.49 \\
\hline 55 & P16870 & CBPE & YES & 0.0000 & 0.29 \\
\hline 56 & P17936 & IBP3 & YES & 0.3626 & 1.18 \\
\hline 57 & P19022 & CADH2 & YES & 0.0000 & 0.36 \\
\hline 58 & P23142 & FBLN1 & YES & 0.1663 & 0.84 \\
\hline 59 & P24821 & TENA & YES & 0.6090 & 1.07 \\
\hline 60 & P27169 & PON1 & YES & 0.0000 & 14.76 \\
\hline 61 & P30041 & PRDX6 & YES & 0.0001 & 0.64 \\
\hline 62 & P30086 & PEBP1 & YES & 0.0201 & 0.81 \\
\hline 63 & P30530 & UFO & YES & 0.0000 & 0.48 \\
\hline 64 & P32119 & PRDX2 & YES & 0.1965 & 1.32 \\
\hline 65 & P33908 & MA1A1 & YES & 0.0027 & 0.63 \\
\hline 66 & P35052 & GPC1 & YES & 0.5471 & 0.82 \\
\hline 67 & P35527 & K1C9 & YES & 0.0000 & 1.94 \\
\hline 68 & P35555 & FBN1 & YES & 0.0226 & 0.57 \\
\hline 69 & P35908 & K22E & YES & 0.0001 & 1.63 \\
\hline 70 & P37802 & TAGL2 & YES & 0.0000 & 2.18 \\
\hline 71 & P40925 & MDHC & YES & 0.6026 & 0.90 \\
\hline 72 & P42785 & PCP & YES & 0.0000 & 0.38 \\
\hline 73 & P50454 & SERPH & YES & 0.0000 & 5.27 \\
\hline 74 & P50895 & BCAM & YES & 0.5800 & 1.18 \\
\hline 75 & P52735 & VAV2 & YES & 0.0048 & 0.46 \\
\hline 76 & P53634 & CATC & YES & 0.0000 & 0.35 \\
\hline 77 & P60174 & TPIS & YES & 0.0287 & 0.71 \\
\hline 78 & P61769 & B2MG & YES & 0.0000 & 0.41 \\
\hline 79 & P61970 & NTF2 & YES & 0.0000 & 0.63 \\
\hline 80 & P62158 & CALM & YES & 0.0631 & 1.12 \\
\hline 81 & P62805 & $\mathrm{H} 4$ & YES & 0.0000 & 4.54 \\
\hline 82 & P68871 & HBB & YES & 0.0000 & 11.37 \\
\hline 83 & P78417 & GSTO1 & YES & 0.0007 & 0.55 \\
\hline 84 & P80188 & NGAL & YES & 0.0000 & 3.07 \\
\hline 85 & P98160 & PGBM & YES & 0.0000 & 0.50 \\
\hline 86 & Q01082 & SPTB2 & YES & 0.2065 & 0.83 \\
\hline 87 & Q02818 & NUCB1 & YES & 0.0155 & 0.72 \\
\hline 88 & Q03154 & ACY1 & YES & 0.0000 & 0.52 \\
\hline 89 & Q08629 & TICN1 & YES & 0.0728 & 1.54 \\
\hline 90 & Q12805 & FBLN3 & YES & 0.0000 & 0.37 \\
\hline 91 & Q12841 & FSTL1 & YES & 0.1357 & 1.35 \\
\hline 92 & Q14019 & COTL1 & YES & 0.0000 & 0.27 \\
\hline 93 & Q14126 & DSG2 & YES & 0.0843 & 0.72 \\
\hline 94 & Q14508 & WFDC2 & YES & 0.0000 & 0.44 \\
\hline 95 & A0M8Q6 & LAC7 & NO & 0.0000 & 1.15 \\
\hline 96 & B9A064 & IGLL5 & $\mathrm{NO}$ & 0.0000 & 0.59 \\
\hline 97 & O00115 & DNS2A & $\mathrm{NO}$ & 0.3908 & 0.82 \\
\hline 98 & O00187 & MASP2 & $\mathrm{NO}$ & 0.0000 & 0.48 \\
\hline 99 & O00391 & QSOX1 & $\mathrm{NO}$ & 0.2147 & 0.89 \\
\hline 100 & O00533 & NCHL1 & $\mathrm{NO}$ & 0.0031 & 0.48 \\
\hline 101 & O00584 & RNT2 & $\mathrm{NO}$ & 0.0000 & 0.50 \\
\hline 102 & O14745 & NHRF1 & $\mathrm{NO}$ & 0.0546 & 0.62 \\
\hline 103 & O14773 & TPP1 & NO & 0.0000 & 0.27 \\
\hline 104 & O14841 & OPLA & $\mathrm{NO}$ & 0.0113 & 1.62 \\
\hline
\end{tabular}




\begin{tabular}{|c|c|c|c|c|c|}
\hline 105 & O14981 & BTAF1 & $\mathrm{NO}$ & 0.0000 & 0.35 \\
\hline 106 & $\mathrm{O} 43278$ & SPIT1 & NO & 0.4282 & 0.85 \\
\hline 107 & O43291 & SPIT2 & $\mathrm{NO}$ & 0.0032 & 0.44 \\
\hline 108 & O43451 & MGA & $\mathrm{NO}$ & 0.0000 & 0.38 \\
\hline 109 & O43490 & PROM1 & $\mathrm{NO}$ & 0.0000 & 0.24 \\
\hline 110 & O43505 & B4GA1 & $\mathrm{NO}$ & 0.8536 & 0.95 \\
\hline 111 & $\mathrm{O} 43653$ & PSCA & $\mathrm{NO}$ & 0.0000 & 10.64 \\
\hline 112 & O43707 & ACTN4 & $\mathrm{NO}$ & 0.1008 & 1.31 \\
\hline 113 & O43847 & NRDC & $\mathrm{NO}$ & 0.0557 & 0.41 \\
\hline 114 & O43866 & CD5L & $\mathrm{NO}$ & 0.0802 & 1.11 \\
\hline 115 & O60494 & CUBN & $\mathrm{NO}$ & 0.0000 & 0.47 \\
\hline 116 & O60635 & TSN1 & $\mathrm{NO}$ & 0.0000 & 0.34 \\
\hline 117 & O60888 & CUTA & $\mathrm{NO}$ & 0.0000 & 0.46 \\
\hline 118 & O75144 & ICOSL & $\mathrm{NO}$ & 0.0000 & 0.27 \\
\hline 119 & O75309 & CAD16 & $\mathrm{NO}$ & 0.0038 & 0.59 \\
\hline 120 & O75340 & PDCD6 & $\mathrm{NO}$ & 0.0008 & 0.67 \\
\hline 121 & O75368 & SH3L1 & $\mathrm{NO}$ & 0.0187 & 0.75 \\
\hline 122 & O75594 & PGRP1 & $\mathrm{NO}$ & 0.0000 & 1.98 \\
\hline 123 & O75629 & CREG1 & $\mathrm{NO}$ & 0.0002 & 0.30 \\
\hline 124 & O75718 & CRTAP & $\mathrm{NO}$ & 0.2323 & 0.83 \\
\hline 125 & O75874 & IDHC & $\mathrm{NO}$ & 0.0023 & 0.54 \\
\hline 126 & O75882 & ATRN & $\mathrm{NO}$ & 0.0116 & 0.80 \\
\hline 127 & O94919 & ENDD1 & $\mathrm{NO}$ & 0.8919 & 0.98 \\
\hline 128 & O95274 & LYPD3 & $\mathrm{NO}$ & 0.0200 & 2.71 \\
\hline 129 & O95613 & PCNT & NO & 0.0000 & 1.55 \\
\hline 130 & O95865 & DDAH2 & $\mathrm{NO}$ & 0.0559 & 0.67 \\
\hline 131 & O96009 & NAPSA & $\mathrm{NO}$ & 0.0000 & 0.25 \\
\hline 132 & P00352 & AL1A1 & $\mathrm{NO}$ & 0.0120 & 0.62 \\
\hline 133 & P00450 & CERU & $\mathrm{NO}$ & 0.0000 & 2.06 \\
\hline 134 & P00491 & PNPH & NO & 0.8563 & 0.97 \\
\hline 135 & P00734 & THRB & $\mathrm{NO}$ & 0.8536 & 0.98 \\
\hline 136 & P00738 & HPT & NO & 0.0000 & 11.92 \\
\hline 137 & P00747 & PLMN & $\mathrm{NO}$ & 0.0000 & 1.69 \\
\hline 138 & P00748 & FA12 & $\mathrm{NO}$ & 0.0001 & 2.73 \\
\hline 139 & P00749 & UROK & $\mathrm{NO}$ & 0.2790 & 0.73 \\
\hline 140 & P00751 & CFAB & $\mathrm{NO}$ & 0.0000 & 2.10 \\
\hline 141 & P00915 & CAH1 & $\mathrm{NO}$ & 0.0000 & 2.09 \\
\hline 142 & P00918 & CAH2 & $\mathrm{NO}$ & 0.0000 & 0.46 \\
\hline 143 & P00995 & ISK1 & $\mathrm{NO}$ & 0.0061 & 0.39 \\
\hline 144 & P01011 & AACT & NO & 0.8151 & 0.98 \\
\hline 145 & P01019 & ANGT & $\mathrm{NO}$ & 0.0000 & 1.59 \\
\hline 146 & P01023 & A2MG & $\mathrm{NO}$ & 0.0000 & 19.17 \\
\hline 147 & P01040 & CYTA & $\mathrm{NO}$ & 0.0001 & 0.37 \\
\hline 148 & P01042 & KNG1 & $\mathrm{NO}$ & 0.0000 & 0.56 \\
\hline 149 & P01591 & IGJ & $\mathrm{NO}$ & 0.0174 & 1.08 \\
\hline 150 & P01597 & KV105 & $\mathrm{NO}$ & 0.1217 & 0.82 \\
\hline 151 & P01610 & KV118 & NO & 0.6765 & 0.96 \\
\hline 152 & P01611 & KV119 & $\mathrm{NO}$ & 0.0408 & 0.64 \\
\hline 153 & P01612 & KV120 & $\mathrm{NO}$ & 0.0000 & 1.64 \\
\hline 154 & P01613 & KV121 & $\mathrm{NO}$ & 0.0000 & 1.70 \\
\hline 155 & P01616 & KV203 & $\mathrm{NO}$ & 0.0000 & 2.89 \\
\hline 156 & P01619 & KV301 & $\mathrm{NO}$ & 0.3609 & 0.95 \\
\hline 157 & P01620 & KV302 & $\mathrm{NO}$ & 0.0112 & 1.66 \\
\hline 158 & P01621 & KV303 & $\mathrm{NO}$ & 0.0000 & 1.34 \\
\hline 159 & P01714 & LV301 & NO & 0.0000 & 2.27 \\
\hline 160 & P01717 & LV403 & $\mathrm{NO}$ & 0.0000 & 3.98 \\
\hline
\end{tabular}




\begin{tabular}{|c|c|c|c|c|c|}
\hline 161 & P01743 & HV102 & NO & 0.0000 & 2.65 \\
\hline 162 & P01763 & HV302 & NO & 0.0000 & 2.76 \\
\hline 163 & P01771 & HV310 & NO & 0.0000 & 2.03 \\
\hline 164 & P01773 & HV312 & NO & 0.0058 & 1.66 \\
\hline 165 & P01781 & HV320 & NO & 0.1811 & 1.27 \\
\hline 166 & P01833 & PIGR & NO & 0.0000 & 0.52 \\
\hline 167 & P01834 & IGKC & NO & 0.0000 & 1.45 \\
\hline 168 & P01857 & IGHG1 & NO & 0.0000 & 4.06 \\
\hline 169 & P01859 & IGHG2 & NO & 0.0037 & 1.67 \\
\hline 170 & P01860 & IGHG3 & NO & 0.0000 & 9.57 \\
\hline 171 & P01861 & IGHG4 & NO & 0.0001 & 3.20 \\
\hline 172 & P01871 & IGHM & NO & 0.0000 & 9.75 \\
\hline 173 & P01876 & IGHA1 & NO & 0.0000 & 1.91 \\
\hline 174 & P01877 & IGHA2 & NO & 0.0000 & 2.76 \\
\hline 175 & P02042 & HBD & NO & 0.0000 & 8.67 \\
\hline 176 & P02452 & CO1A1 & NO & 0.0000 & 0.51 \\
\hline 177 & P02647 & APOA1 & NO & 0.0000 & 14.94 \\
\hline 178 & P02652 & APOA2 & NO & 0.0000 & 22.87 \\
\hline 179 & P02654 & APOC1 & NO & 0.0000 & 6.22 \\
\hline 180 & P02656 & APOC3 & NO & 0.0000 & 3.19 \\
\hline 181 & P02671 & FIBA & NO & 0.0000 & 4.25 \\
\hline 182 & P02675 & FIBB & NO & 0.0000 & 29.84 \\
\hline 183 & P02679 & FIBG & NO & 0.0000 & 25.23 \\
\hline 184 & P02743 & SAMP & NO & 0.0315 & 1.32 \\
\hline 185 & P02748 & $\mathrm{CO} 9$ & NO & 0.0000 & 3.95 \\
\hline 186 & P02749 & APOH & NO & 0.8314 & 1.01 \\
\hline 187 & P02750 & A2GL & NO & 0.0000 & 1.45 \\
\hline 188 & P02753 & RET4 & NO & 0.4197 & 0.96 \\
\hline 189 & P02760 & AMBP & NO & 0.0000 & 0.60 \\
\hline 190 & P02763 & A1AG1 & NO & 0.0000 & 1.21 \\
\hline 191 & P02765 & FETUA & NO & 0.0148 & 0.78 \\
\hline 192 & P02766 & TTHY & NO & 0.0000 & 2.68 \\
\hline 193 & P02774 & VTDB & NO & 0.0000 & 3.47 \\
\hline 194 & P02788 & TRFL & NO & 0.0000 & 3.05 \\
\hline 195 & P02790 & HEMO & NO & 0.0000 & 2.72 \\
\hline 196 & P04004 & VTNC & NO & 0.0000 & 1.76 \\
\hline 197 & P04062 & GLCM & NO & 0.0705 & 0.54 \\
\hline 198 & P04156 & PRIO & NO & 0.0000 & 0.32 \\
\hline 199 & P04180 & LCAT & NO & 0.0000 & 0.42 \\
\hline 200 & P04196 & HRG & NO & 0.0000 & 6.80 \\
\hline 201 & P04211 & LV001 & NO & 0.0000 & 2.21 \\
\hline 202 & P04216 & THY1 & NO & 0.0799 & 0.74 \\
\hline 203 & P04217 & A1BG & NO & 0.0000 & 1.86 \\
\hline 204 & P04430 & KV122 & NO & 0.0264 & 1.42 \\
\hline 205 & P04433 & KV309 & NO & 0.0001 & 1.30 \\
\hline 206 & P04434 & KV310 & NO & 0.0000 & 1.37 \\
\hline 207 & P04746 & AMYP & NO & 0.0000 & 0.63 \\
\hline 208 & P05060 & SCG1 & NO & 0.0174 & 0.51 \\
\hline 209 & P05062 & ALDOB & NO & 0.0000 & 0.44 \\
\hline 210 & P05090 & APOD & NO & 0.0000 & 0.61 \\
\hline 211 & P05109 & S10A8 & NO & 0.0000 & 5.53 \\
\hline 212 & P05154 & IPSP & NO & 0.0000 & 0.46 \\
\hline 213 & P05155 & IC1 & NO & 0.0000 & 1.25 \\
\hline 214 & P05164 & PERM & NO & 0.0000 & 5.98 \\
\hline 215 & P05362 & ICAM1 & NO & 0.2938 & 0.62 \\
\hline 216 & P05451 & REG1A & NO & 0.0000 & 0.70 \\
\hline
\end{tabular}




\begin{tabular}{|c|c|c|c|c|c|}
\hline 217 & P05452 & TETN & $\mathrm{NO}$ & 0.7301 & 0.96 \\
\hline 218 & P05543 & THBG & $\mathrm{NO}$ & 0.0023 & 1.39 \\
\hline 219 & P05546 & HEP2 & $\mathrm{NO}$ & 0.0000 & 3.19 \\
\hline 220 & P05787 & $\mathrm{K} 2 \mathrm{C} 8$ & $\mathrm{NO}$ & 0.4282 & 0.88 \\
\hline 221 & P05937 & CALB1 & $\mathrm{NO}$ & 0.0000 & 0.53 \\
\hline 222 & P06280 & AGAL & $\mathrm{NO}$ & 0.0000 & 0.42 \\
\hline 223 & P06311 & KV311 & $\mathrm{NO}$ & 0.3437 & 0.92 \\
\hline 224 & P06331 & HV209 & $\mathrm{NO}$ & 0.0065 & 1.35 \\
\hline 225 & P06396 & GELS & $\mathrm{NO}$ & 0.1124 & 0.87 \\
\hline 226 & P06702 & S10A9 & $\mathrm{NO}$ & 0.0000 & 3.07 \\
\hline 227 & P06727 & APOA4 & $\mathrm{NO}$ & 0.0047 & 1.47 \\
\hline 228 & P06865 & HEXA & $\mathrm{NO}$ & 0.0000 & 0.58 \\
\hline 229 & P06870 & KLK1 & $\mathrm{NO}$ & 0.0000 & 0.51 \\
\hline 230 & P07108 & ACBP & $\mathrm{NO}$ & 0.0000 & 0.58 \\
\hline 231 & P07204 & TRBM & $\mathrm{NO}$ & 0.2576 & 0.68 \\
\hline 232 & P07288 & KLK3 & $\mathrm{NO}$ & 0.0002 & 0.44 \\
\hline 233 & P07355 & ANXA2 & $\mathrm{NO}$ & 0.3118 & 0.84 \\
\hline 234 & P07602 & SAP & $\mathrm{NO}$ & 0.0000 & 0.41 \\
\hline 235 & P07686 & HEXB & $\mathrm{NO}$ & 0.0000 & 0.58 \\
\hline 236 & P07711 & CATL1 & $\mathrm{NO}$ & 0.0001 & 0.62 \\
\hline 237 & P07911 & UROM & $\mathrm{NO}$ & 0.0000 & 0.29 \\
\hline 238 & P07998 & RNAS1 & $\mathrm{NO}$ & 0.0000 & 0.48 \\
\hline 239 & P08133 & ANXA6 & $\mathrm{NO}$ & 0.8178 & 1.06 \\
\hline 240 & P08174 & DAF & $\mathrm{NO}$ & 0.0000 & 0.37 \\
\hline 241 & P08185 & CBG & $\mathrm{NO}$ & 0.8879 & 1.02 \\
\hline 242 & P08195 & $4 \mathrm{~F} 2$ & $\mathrm{NO}$ & 0.2147 & 0.84 \\
\hline 243 & P08236 & BGLR & $\mathrm{NO}$ & 0.0000 & 0.20 \\
\hline 244 & P08294 & SODE & $\mathrm{NO}$ & 0.0002 & 0.62 \\
\hline 245 & P08473 & NEP & $\mathrm{NO}$ & 0.0000 & 0.37 \\
\hline 246 & P08519 & APOA & $\mathrm{NO}$ & 0.0000 & 0.73 \\
\hline 247 & P08571 & CD14 & $\mathrm{NO}$ & 0.0000 & 0.41 \\
\hline 248 & P08582 & TRFM & $\mathrm{NO}$ & 0.0631 & 0.52 \\
\hline 249 & P08603 & CFAH & $\mathrm{NO}$ & 0.0000 & 9.39 \\
\hline 250 & P08637 & FCG3A & $\mathrm{NO}$ & 0.0000 & 0.48 \\
\hline 251 & P08697 & A2AP & $\mathrm{NO}$ & 0.0005 & 1.67 \\
\hline 252 & P08758 & ANXA5 & $\mathrm{NO}$ & 0.0000 & 0.51 \\
\hline 253 & P09467 & F16P1 & $\mathrm{NO}$ & 0.0000 & 0.56 \\
\hline 254 & P09525 & ANXA4 & $\mathrm{NO}$ & 0.0000 & 0.36 \\
\hline 255 & P09619 & PGFRB & $\mathrm{NO}$ & 0.0014 & 0.55 \\
\hline 256 & P09871 & $\mathrm{C} 1 \mathrm{~S}$ & $\mathrm{NO}$ & 0.0000 & 1.92 \\
\hline 257 & P0C0L4 & CO4A & $\mathrm{NO}$ & 0.0011 & 2.30 \\
\hline 258 & P0C0L5 & CO4B & $\mathrm{NO}$ & 0.0065 & 1.43 \\
\hline 259 & P10153 & RNAS2 & $\mathrm{NO}$ & 0.0000 & 0.39 \\
\hline 260 & P10451 & OSTP & $\mathrm{NO}$ & 0.0000 & 0.37 \\
\hline 261 & P10599 & THIO & $\mathrm{NO}$ & 0.0000 & 0.60 \\
\hline 262 & P10619 & PPGB & $\mathrm{NO}$ & 0.0000 & 0.29 \\
\hline 263 & P10643 & $\mathrm{CO} 7$ & $\mathrm{NO}$ & 0.0023 & 1.59 \\
\hline 264 & P10645 & CMGA & $\mathrm{NO}$ & 0.0052 & 0.55 \\
\hline 265 & P10909 & CLUS & $\mathrm{NO}$ & 0.0000 & 0.72 \\
\hline 266 & P11021 & GRP78 & $\mathrm{NO}$ & 0.0471 & 1.46 \\
\hline 267 & P11117 & PPAL & $\mathrm{NO}$ & 0.0000 & 0.36 \\
\hline 268 & P11142 & HSP7C & $\mathrm{NO}$ & 0.0308 & 0.82 \\
\hline 269 & P11216 & PYGB & $\mathrm{NO}$ & 0.0000 & 0.17 \\
\hline 270 & P11279 & LAMP1 & $\mathrm{NO}$ & 0.0000 & 0.32 \\
\hline 271 & P12111 & CO6A3 & $\mathrm{NO}$ & 0.0288 & 0.75 \\
\hline 272 & P12259 & FA5 & $\mathrm{NO}$ & 0.0010 & 1.88 \\
\hline
\end{tabular}




\begin{tabular}{|c|c|c|c|c|c|}
\hline 273 & P12273 & PIP & $\mathrm{NO}$ & 0.0288 & 0.77 \\
\hline 274 & P12429 & ANXA3 & NO & 0.0012 & 1.90 \\
\hline 275 & P12821 & ACE & $\mathrm{NO}$ & 0.0002 & 0.48 \\
\hline 276 & P13473 & LAMP2 & NO & 0.0000 & 0.34 \\
\hline 277 & P13489 & RINI & NO & 0.0000 & 3.03 \\
\hline 278 & P13598 & ICAM2 & $\mathrm{NO}$ & 0.3437 & 1.30 \\
\hline 279 & P13645 & K1C10 & NO & 0.0000 & 1.50 \\
\hline 280 & P13671 & CO6 & $\mathrm{NO}$ & 0.0000 & 4.41 \\
\hline 281 & P13688 & CEAM1 & NO & 0.0000 & 0.32 \\
\hline 282 & P13797 & PLST & $\mathrm{NO}$ & 0.9388 & 1.02 \\
\hline 283 & P13987 & CD59 & NO & 0.0000 & 0.39 \\
\hline 284 & P14384 & СВРM & NO & 0.0007 & 0.64 \\
\hline 285 & P14550 & AK1A1 & NO & 0.0000 & 0.43 \\
\hline 286 & P14780 & MMP9 & NO & 0.0000 & 12.98 \\
\hline 287 & P15144 & AMPN & NO & 0.0000 & 0.38 \\
\hline 288 & P15289 & ARSA & NO & 0.0000 & 0.48 \\
\hline 289 & P15291 & B4GT1 & NO & 0.7089 & 1.06 \\
\hline 290 & P15309 & PPAP & NO & 0.0000 & 0.43 \\
\hline 291 & P15586 & GNS & NO & 0.0000 & 0.33 \\
\hline 292 & P15814 & IGLL1 & NO & 0.1151 & 1.73 \\
\hline 293 & P15848 & ARSB & NO & 0.0000 & 0.38 \\
\hline 294 & P15941 & MUC1 & NO & 0.0000 & 0.45 \\
\hline 295 & P16109 & LYAM3 & NO & 0.0023 & 2.12 \\
\hline 296 & P16278 & BGAL & NO & 0.0000 & 0.26 \\
\hline 297 & P16284 & PECA1 & NO & 0.0000 & 0.60 \\
\hline 298 & P16444 & DPEP1 & NO & 0.0761 & 0.80 \\
\hline 299 & P17050 & NAGAB & NO & 0.0000 & 0.60 \\
\hline 300 & P17405 & ASM & NO & 0.2921 & 1.54 \\
\hline 301 & P17844 & DDX5 & NO & 0.0000 & 2.45 \\
\hline 302 & P17900 & SAP3 & NO & 0.0000 & 0.35 \\
\hline 303 & P17931 & LEG3 & NO & 0.4784 & 0.89 \\
\hline 304 & P18065 & IBP2 & NO & 0.0081 & 0.57 \\
\hline 305 & P18428 & LBP & NO & 0.0000 & 2.35 \\
\hline 306 & P18827 & SDC1 & NO & 0.0902 & 0.64 \\
\hline 307 & P19256 & LFA3 & NO & 0.0000 & 0.56 \\
\hline 308 & P19320 & VCAM1 & NO & 0.0000 & 0.40 \\
\hline 309 & P19652 & A1AG2 & NO & 0.0013 & 0.83 \\
\hline 310 & P19801 & AOC1 & NO & 0.0693 & 0.69 \\
\hline 311 & P19823 & ITIH2 & NO & 0.0000 & 7.31 \\
\hline 312 & P19827 & ITIH1 & NO & 0.0000 & 2.90 \\
\hline 313 & P19961 & AMY2B & NO & 0.0487 & 0.79 \\
\hline 314 & P20742 & PZP & NO & 0.0000 & 19.27 \\
\hline 315 & P20933 & ASPG & NO & 0.0000 & 0.53 \\
\hline 316 & P21266 & GSTM3 & NO & 0.0800 & 0.60 \\
\hline 317 & P21333 & FLNA & $\mathrm{NO}$ & 0.0000 & 0.36 \\
\hline 318 & P21399 & ACOC & NO & 0.0040 & 0.51 \\
\hline 319 & P21810 & PGS1 & $\mathrm{NO}$ & 0.0546 & 0.60 \\
\hline 320 & P22105 & TENX & $\mathrm{NO}$ & 0.0198 & 0.70 \\
\hline 321 & P22352 & GPX3 & $\mathrm{NO}$ & 0.3672 & 1.07 \\
\hline 322 & P22792 & CPN2 & NO & 0.0957 & 0.79 \\
\hline 323 & P22891 & PROZ & $\mathrm{NO}$ & 0.0000 & 0.42 \\
\hline 324 & P23083 & HV103 & NO & 0.0000 & 2.20 \\
\hline 325 & P23284 & PPIB & NO & 0.0599 & 1.69 \\
\hline 326 & P23396 & RS3 & NO & 0.5860 & 1.09 \\
\hline 327 & P23470 & PTPRG & $\mathrm{NO}$ & 0.4962 & 0.87 \\
\hline 328 & P24158 & PRTN3 & $\mathrm{NO}$ & 0.0000 & 6.73 \\
\hline
\end{tabular}




\begin{tabular}{|c|c|c|c|c|c|}
\hline 329 & P24855 & DNAS1 & $\mathrm{NO}$ & 0.0000 & 0.41 \\
\hline 330 & P25311 & ZA2G & NO & 0.0000 & 0.45 \\
\hline 331 & P25325 & THTM & $\mathrm{NO}$ & 0.0164 & 0.56 \\
\hline 332 & P25815 & S100P & NO & 0.2988 & 0.85 \\
\hline 333 & P26038 & MOES & NO & 0.0320 & 1.19 \\
\hline 334 & P26358 & DNMT1 & NO & 0.5287 & 1.23 \\
\hline 335 & P27105 & STOM & $\mathrm{NO}$ & 0.0002 & 0.40 \\
\hline 336 & P27348 & $1433 \mathrm{~T}$ & NO & 0.6040 & 0.88 \\
\hline 337 & P27487 & DPP4 & NO & 0.0000 & 0.34 \\
\hline 338 & P28799 & GRN & NO & 0.0000 & 0.33 \\
\hline 339 & P29279 & CTGF & $\mathrm{NO}$ & 0.0000 & 0.27 \\
\hline 340 & P29401 & TKT & NO & 0.6972 & 1.10 \\
\hline 341 & P29622 & KAIN & $\mathrm{NO}$ & 0.8739 & 1.03 \\
\hline 342 & P29966 & MARCS & NO & 0.0000 & 0.19 \\
\hline 343 & P29972 & AQP1 & $\mathrm{NO}$ & 0.0000 & 0.41 \\
\hline 344 & P30043 & BLVRB & NO & 0.0008 & 0.43 \\
\hline 345 & P30044 & PRDX5 & NO & 0.9991 & 1.00 \\
\hline 346 & P30047 & GFRP & NO & 0.0030 & 0.47 \\
\hline 347 & P30085 & $\mathrm{KCY}$ & NO & 0.0173 & 0.54 \\
\hline 348 & P31949 & S10AB & NO & 0.0032 & 1.83 \\
\hline 349 & P32004 & L1CAM & NO & 0.5965 & 0.89 \\
\hline 350 & P34059 & GALNS & NO & 0.0000 & 0.41 \\
\hline 351 & P35443 & TSP4 & $\mathrm{NO}$ & 0.0139 & 0.68 \\
\hline 352 & P35754 & GLRX1 & NO & 0.0000 & 0.52 \\
\hline 353 & P35858 & ALS & NO & 0.0000 & 1.93 \\
\hline 354 & P36639 & 8ODP & NO & 0.5860 & 1.20 \\
\hline 355 & P36955 & PEDF & NO & 0.0000 & 1.93 \\
\hline 356 & P36957 & ODO2 & NO & 0.0000 & 0.42 \\
\hline 357 & P37837 & TALDO & NO & 0.0000 & 3.16 \\
\hline 358 & P38606 & VATA & NO & 0.0007 & 0.43 \\
\hline 359 & P39059 & COFA1 & NO & 0.0013 & 0.67 \\
\hline 360 & P39060 & COIA1 & NO & 0.1663 & 0.85 \\
\hline 361 & P40189 & IL6RB & $\mathrm{NO}$ & 0.0006 & 0.78 \\
\hline 362 & P40227 & TCPZ & $\mathrm{NO}$ & 0.0019 & 0.76 \\
\hline 363 & P41222 & PTGDS & NO & 0.0000 & 0.59 \\
\hline 364 & P43121 & MUC18 & NO & 0.3024 & 1.31 \\
\hline 365 & P43251 & BTD & NO & 0.0000 & 0.61 \\
\hline 366 & P43652 & AFAM & NO & 0.0000 & 2.04 \\
\hline 367 & P45877 & PPIC & NO & 0.5683 & 0.80 \\
\hline 368 & P49913 & CAMP & NO & 0.0000 & 33.84 \\
\hline 369 & P50897 & PPT1 & $\mathrm{NO}$ & 0.0079 & 0.42 \\
\hline 370 & P50995 & ANX11 & NO & 0.0000 & 0.49 \\
\hline 371 & P51688 & SPHM & NO & 0.0000 & 0.32 \\
\hline 372 & P51884 & LUM & NO & 0.0198 & 1.33 \\
\hline 373 & P52597 & HNRPF & NO & 0.0036 & 0.47 \\
\hline 374 & P52758 & UK114 & $\mathrm{NO}$ & 0.0000 & 0.34 \\
\hline 375 & P53990 & IST1 & $\mathrm{NO}$ & 0.0000 & 0.54 \\
\hline 376 & P54760 & EPHB4 & NO & 0.0000 & 0.34 \\
\hline 377 & P54802 & ANAG & $\mathrm{NO}$ & 0.0000 & 0.45 \\
\hline 378 & P55000 & SLUR1 & NO & 0.0000 & 0.34 \\
\hline 379 & P55060 & XPO2 & NO & 0.0008 & 2.05 \\
\hline 380 & P55083 & MFAP4 & NO & 0.7195 & 1.15 \\
\hline 381 & P55285 & CADH6 & NO & 0.0016 & 0.44 \\
\hline 382 & P55290 & CAD13 & NO & 0.0000 & 0.52 \\
\hline 383 & P55957 & BID & NO & 0.0013 & 0.63 \\
\hline 384 & P56537 & IF6 & NO & 0.0105 & 0.45 \\
\hline
\end{tabular}




\begin{tabular}{|c|c|c|c|c|c|}
\hline 385 & P60022 & DEFB1 & $\mathrm{NO}$ & 0.0000 & 0.27 \\
\hline 386 & P61626 & LYSC & NO & 0.0000 & 3.47 \\
\hline 387 & P61916 & NPC2 & $\mathrm{NO}$ & 0.0000 & 0.43 \\
\hline 388 & P61981 & $1433 \mathrm{G}$ & $\mathrm{NO}$ & 0.0000 & 0.47 \\
\hline 389 & P62258 & $1433 \mathrm{E}$ & $\mathrm{NO}$ & 0.0986 & 1.35 \\
\hline 390 & P62937 & PPIA & $\mathrm{NO}$ & 0.0094 & 1.28 \\
\hline 391 & P63104 & $1433 Z$ & $\mathrm{NO}$ & 0.4086 & 1.21 \\
\hline 392 & P68402 & PA1B2 & $\mathrm{NO}$ & 0.6328 & 1.06 \\
\hline 393 & P69905 & HBA & $\mathrm{NO}$ & 0.0000 & 16.27 \\
\hline 394 & P80723 & BASP1 & $\mathrm{NO}$ & 0.0000 & 0.22 \\
\hline 395 & P80748 & LV302 & $\mathrm{NO}$ & 0.0000 & 1.72 \\
\hline 396 & P98164 & LRP2 & $\mathrm{NO}$ & 0.0000 & 0.37 \\
\hline 397 & P98172 & EFNB1 & $\mathrm{NO}$ & 0.0109 & 0.55 \\
\hline 398 & P99999 & CYC & $\mathrm{NO}$ & 0.0343 & 2.06 \\
\hline 399 & Q00796 & DHSO & $\mathrm{NO}$ & 0.0003 & 0.36 \\
\hline 400 & Q01459 & DIAC & $\mathrm{NO}$ & 0.0000 & 0.34 \\
\hline 401 & Q01518 & CAP1 & $\mathrm{NO}$ & 0.0010 & 1.46 \\
\hline 402 & Q02413 & DSG1 & $\mathrm{NO}$ & 0.4326 & 1.12 \\
\hline 403 & Q02487 & DSC2 & $\mathrm{NO}$ & 0.0000 & 0.42 \\
\hline 404 & Q03403 & TFF2 & $\mathrm{NO}$ & 0.0000 & 0.50 \\
\hline 405 & Q05707 & COEA1 & $\mathrm{NO}$ & 0.0000 & 0.29 \\
\hline 406 & Q06830 & PRDX1 & $\mathrm{NO}$ & 0.4922 & 0.93 \\
\hline 407 & Q07075 & AMPE & $\mathrm{NO}$ & 0.0000 & 0.55 \\
\hline 408 & Q07507 & DERM & $\mathrm{NO}$ & 0.0000 & 0.54 \\
\hline 409 & Q07654 & TFF3 & NO & 0.0000 & 0.38 \\
\hline 410 & Q08174 & PCDH1 & NO & 0.0761 & 0.72 \\
\hline 411 & Q08380 & LG3BP & $\mathrm{NO}$ & 0.0000 & 0.49 \\
\hline 412 & Q10588 & BST1 & $\mathrm{NO}$ & 0.0101 & 0.79 \\
\hline 413 & Q12765 & SCRN1 & NO & 0.0004 & 0.33 \\
\hline 414 & Q12860 & CNTN1 & $\mathrm{NO}$ & 0.0000 & 0.40 \\
\hline 415 & Q12907 & LMAN2 & $\mathrm{NO}$ & 0.0000 & 1.38 \\
\hline 416 & Q12913 & PTPRJ & $\mathrm{NO}$ & 0.0004 & 0.54 \\
\hline 417 & Q13228 & SBP1 & $\mathrm{NO}$ & 0.2628 & 0.83 \\
\hline 418 & Q13308 & PTK7 & $\mathrm{NO}$ & 0.3626 & 0.70 \\
\hline 419 & Q13510 & ASAH1 & $\mathrm{NO}$ & 0.0000 & 0.48 \\
\hline 420 & Q13621 & S12A1 & $\mathrm{NO}$ & 0.0001 & 0.34 \\
\hline 421 & Q14118 & DAG1 & $\mathrm{NO}$ & 0.0187 & 0.66 \\
\hline 422 & Q14247 & SRC8 & $\mathrm{NO}$ & 0.0161 & 0.69 \\
\hline 423 & Q14315 & FLNC & $\mathrm{NO}$ & 0.0080 & 0.64 \\
\hline 424 & Q14515 & SPRL1 & $\mathrm{NO}$ & 0.0003 & 1.67 \\
\hline 425 & Q14624 & ITIH4 & $\mathrm{NO}$ & 0.0118 & 1.20 \\
\hline 426 & Q14914 & PTGR1 & $\mathrm{NO}$ & 0.0000 & 0.23 \\
\hline 427 & Q15113 & PCOC1 & $\mathrm{NO}$ & 0.0000 & 0.41 \\
\hline
\end{tabular}


Table S9. The list of urinary proteins screened by SWATH for MRM verification.

\begin{tabular}{|c|c|c|c|c|c|}
\hline NO. & $\begin{array}{l}\text { Swissprot } \\
\text { number }\end{array}$ & $\begin{array}{l}\text { Protein } \\
\text { description }\end{array}$ & Protein name & $\begin{array}{l}\text { Differerntial } \\
\text { analysis } \\
\text { source }\end{array}$ & $\begin{array}{l}\text { Unique } \\
\text { peptide } \\
\text { number }\end{array}$ \\
\hline 1 & P68871 & Hemoglobin subunit beta & HBB & $2 \mathrm{DE}$ & 2 \\
\hline 2 & P01024 & Complement $\mathrm{C} 3$ & $\mathrm{CO} 3$ & iTRAQ & 5 \\
\hline 3 & P07195 & $\begin{array}{c}\text { L-lactate dehydrogenase } \\
\text { B chain }\end{array}$ & LDHB & iTRAQ & 3 \\
\hline 4 & P02768 & Serum albumin & ALBU & $2 \mathrm{DE}$ & 10 \\
\hline 5 & P98160 & $\begin{array}{l}\text { Basement membrane- } \\
\text { specific heparan sulfate } \\
\text { proteoglycan core protein }\end{array}$ & PGBM & $\begin{array}{l}\text { 2DE and } \\
\text { iTRAQ }\end{array}$ & 6 \\
\hline 6 & P02751 & Fibronectin & FINC & $\begin{array}{l}\text { 2DE and } \\
\text { iTRAO }\end{array}$ & 5 \\
\hline 7 & P12830 & Cadherin-1 & CADH1 & iTRAQ & 3 \\
\hline 8 & P42785 & $\begin{array}{l}\text { Lysosomal Pro-X } \\
\text { carboxypeptidase }\end{array}$ & PCP & iTRAQ & 3 \\
\hline 9 & P16070 & CD44 antigen & CD44 & iTRAQ & 2 \\
\hline 10 & P16870 & Carboxypeptidase E & CBPE & iTRAQ & 2 \\
\hline 11 & P02649 & Apolipoprotein E & APOE & $2 \mathrm{DE}$ & 3 \\
\hline 12 & P05156 & Complement factor I & CFAI & ITRAQ & 5 \\
\hline 13 & P12109 & $\begin{array}{l}\text { Collagen alpha-1(VI) } \\
\text { chain }\end{array}$ & CO6A1 & iTRAQ & 3 \\
\hline 14 & P04264 & $\begin{array}{l}\text { Keratin, type II } \\
\text { cytoskeletal } 1\end{array}$ & $\mathrm{~K} 2 \mathrm{C} 1$ & iTRAQ & 2 \\
\hline 15 & P10253 & $\begin{array}{l}\text { Lysosomal alpha- } \\
\text { glucosidase }\end{array}$ & LYAG & iTRAQ & 7 \\
\hline 16 & P01009 & Alpha-1-antitrypsin & A1AT & iTRAQ & 3 \\
\hline 17 & P07339 & Cathepsin D & CATD & $2 \mathrm{DE}$ & 5 \\
\hline 18 & O95336 & $\begin{array}{c}6- \\
\text { phosphogluconolactonase }\end{array}$ & 6PGL & $\begin{array}{l}\text { 2DE and } \\
\text { iTRAQ }\end{array}$ & 3 \\
\hline 19 & P00338 & $\begin{array}{c}\text { L-lactate dehydrogenase } \\
\text { A chain }\end{array}$ & LDHA & iTRAQ & 3 \\
\hline 20 & P00966 & $\begin{array}{l}\text { Argininosuccinate } \\
\text { synthase }\end{array}$ & ASSY & iTRAQ & 4 \\
\hline 21 & P04083 & Annexin A1 & ANXA1 & ITRAQ & 2 \\
\hline 22 & P04792 & Heat shock protein beta-1 & HSPB1 & $\begin{array}{l}\text { 2DE and } \\
\text { iTRAQ }\end{array}$ & 3 \\
\hline 23 & P07737 & Profilin-1 & PROF1 & $\begin{array}{l}\text { 2DE and } \\
\text { iTRAQ }\end{array}$ & 2 \\
\hline 24 & P13796 & Plastin-2 & PLSL & ITRAQ & 3 \\
\hline 25 & P14543 & Nidogen-1 & NID1 & ITRAQ & 3 \\
\hline 26 & P23142 & Fibulin-1 & FBLN1 & ITRAQ & 3 \\
\hline 27 & P40925 & $\begin{array}{c}\text { Malate dehydrogenase, } \\
\text { cytoplasmic }\end{array}$ & MDHC & $2 \mathrm{DE}$ & 2 \\
\hline 28 & P61970 & Nuclear transport factor 2 & NTF2 & $\begin{array}{l}\text { 2DE and } \\
\text { iTRAQ }\end{array}$ & 3 \\
\hline 29 & Q03154 & $\begin{array}{l}\text { Aminoacylase-1 } \\
\text { EGF-containing fibulin- }\end{array}$ & ACY1 & ITRAQ & 4 \\
\hline 30 & Q12805 & $\begin{array}{l}\text { like extracellular matrix } \\
\text { protein } 1\end{array}$ & FBLN3 & iTRAQ & 3 \\
\hline 31 & Q14126 & Desmoglein-2 & DSG2 & iTRAQ & 2 \\
\hline
\end{tabular}


Table S10. Transitions list of the final 17 BCa related proteins for MRM.

\begin{tabular}{|c|c|}
\hline NO. & Transitions list \\
\hline 1 & P01024.DFDFVPPVVR.+2b3.light \\
\hline 2 & P01024.DFDFVPPVVR.+2b4.light \\
\hline 3 & P01024.DFDFVPPVVR.+2y3.light \\
\hline 4 & P01024.DFDFVPPVVR.+2y5.light \\
\hline 5 & P01024.DFDFVPPVVR.+2y6.light \\
\hline 6 & P01024.QPSSAFAAFVK.+2y4.light \\
\hline 7 & P01024.QPSSAFAAFVK.+2y5.light \\
\hline 8 & P01024.QPSSAFAAFVK.+2y6.light \\
\hline 9 & P01024.QPSSAFAAFVK.+2y7.light \\
\hline 10 & P01024.QPSSAFAAFVK. $+2 \mathrm{y} 8$.light \\
\hline 11 & P01024.SSLSVPYVIVPLK.+2b5.light \\
\hline 12 & P01024.SSLSVPYVIVPLK. $+2 \mathrm{y} 10$.light \\
\hline 13 & P01024.SSLSVPYVIVPLK.+2y4.light \\
\hline 14 & P01024.SSLSVPYVIVPLK.+2y8.light \\
\hline 15 & P01024.SSLSVPYVIVPLK. $+2 \mathrm{y} 9$.light \\
\hline 16 & P02649.SELEEQLTPVAEETR.+2b7.light \\
\hline 17 & P02649.SELEEQLTPVAEETR.+2b8.light \\
\hline 18 & P02649.SELEEQLTPVAEETR.+2y5.light \\
\hline 19 & P02649.SELEEQLTPVAEETR.+2y7.light \\
\hline 20 & P02649.SELEEQLTPVAEETR.+2y9.light \\
\hline 21 & P02649.WELALGR.+2b3.light \\
\hline 22 & P02649.WELALGR.+2b4.light \\
\hline 23 & P02649.WELALGR.+2b5.light \\
\hline 24 & P02649.WELALGR.+2y5.light \\
\hline 25 & P02649.WELALGR.+2y6.light \\
\hline 26 & P02751.STTPDITGYR.+2y6.light \\
\hline 27 & P02751.STTPDITGYR.+2y7.light \\
\hline 28 & P02751.STTPDITGYR.+2y7+2.light \\
\hline 29 & P02751.STTPDITGYR.+2y8.light \\
\hline 30 & P02751.STTPDITGYR.+2y8+2.light \\
\hline 31 & P02751.VDVIPVNLPGEHGQR.+3b4.light \\
\hline 32 & P02751.VDVIPVNLPGEHGQR.+3y11+2.light \\
\hline 33 & P02751.VDVIPVNLPGEHGQR.+3y12+2.light \\
\hline 34 & P02751.VDVIPVNLPGEHGQR.+3y7.light \\
\hline 35 & P02751.VDVIPVNLPGEHGQR.+3y9.light \\
\hline 36 & P02751.VTWAPPPSIDLTNFLVR.+2b4.light \\
\hline 37 & P02751.VTWAPPPSIDLTNFLVR.+2y12+2.light \\
\hline 38 & P02751.VTWAPPPSIDLTNFLVR.+2y13+2.light \\
\hline 39 & P02751.VTWAPPPSIDLTNFLVR.+2y8.light \\
\hline 40 & P02768.FQNALLVR.+2b4.light \\
\hline 41 & P02768.FQNALLVR.+2y4.light \\
\hline 42 & P02768.FQNALLVR.+2y5.light \\
\hline 43 & P02768.FQNALLVR.+2y6.light \\
\hline 44 & P02768.FQNALLVR.+2y7.light \\
\hline 45 & P02768.LVNEVTEFAK.+2y5.light \\
\hline 46 & P02768.LVNEVTEFAK. $+2 \mathrm{y} 6$.light \\
\hline 47 & P02768.LVNEVTEFAK.+2y7.light \\
\hline 48 & P02768.LVNEVTEFAK.+2y8.light \\
\hline 49 & P02768.LVNEVTEFAK.+2y9.light \\
\hline 50 & P04264.SLDLDSIIAEVK.+2y10.light \\
\hline 51 & P04264.SLDLDSIIAEVK.+2y4.light \\
\hline
\end{tabular}




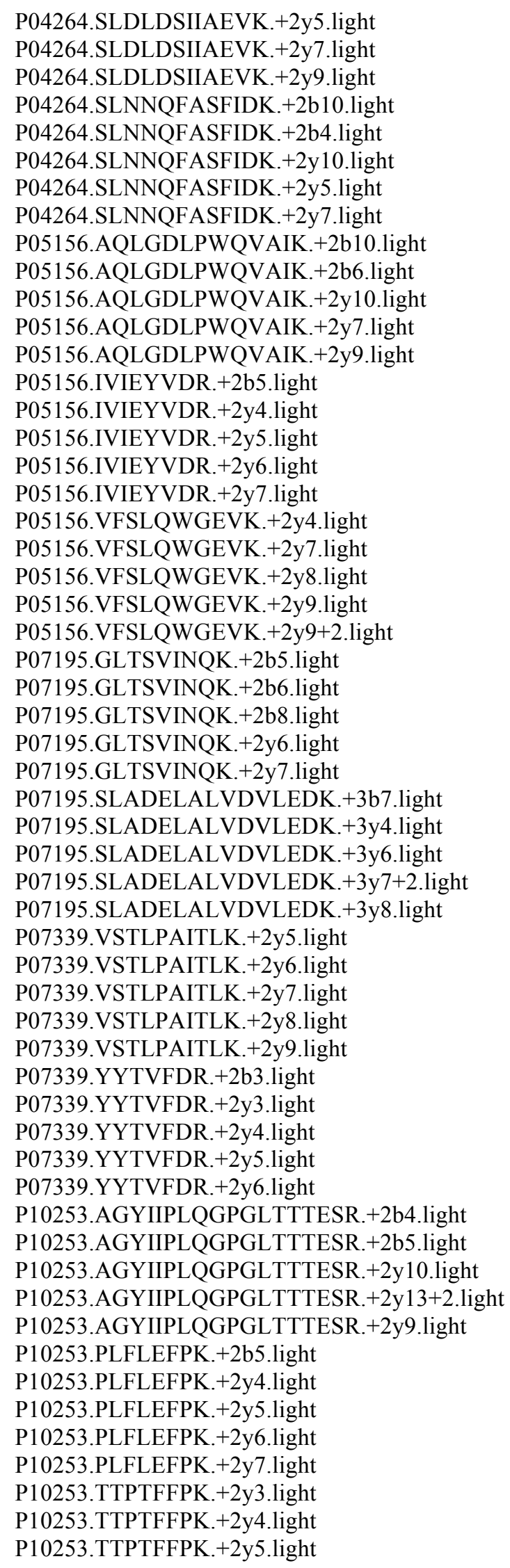




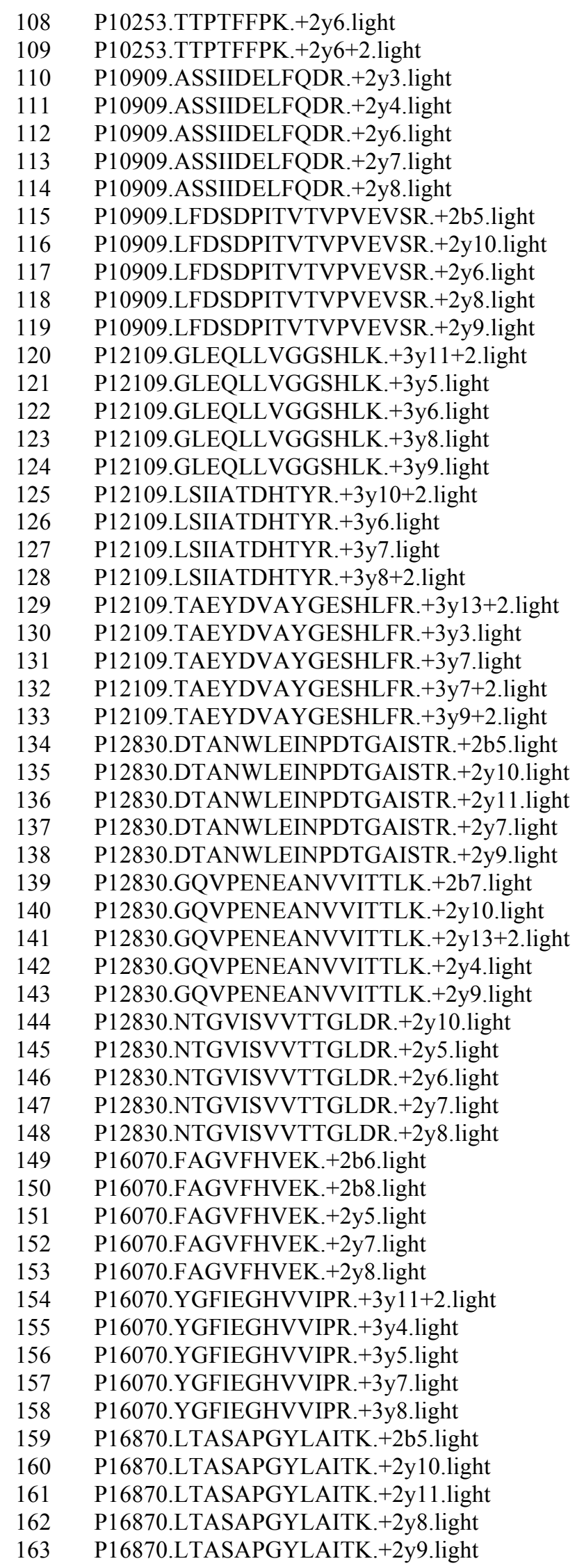




\begin{tabular}{|c|c|}
\hline 164 & P16870.NSLISYLEQIHR.+3y3.light \\
\hline 165 & P16870.NSLISYLEQIHR.+3y4.light \\
\hline 166 & P16870.NSLISYLEQIHR.+3y5.light \\
\hline 167 & P16870.NSLISYLEQIHR.+3y9+2.light \\
\hline 168 & P42785.HLNFLTSEQALADFAELIK.+3b10+2.light \\
\hline 169 & P42785.HLNFLTSEQALADFAELIK.+3b5.light \\
\hline 170 & P42785.HLNFLTSEQALADFAELIK.+3b8.light \\
\hline 171 & P42785.HLNFLTSEQALADFAELIK.+3y4.light \\
\hline 172 & P42785.HLNFLTSEQALADFAELIK.+3y8.light \\
\hline 173 & P42785.YYGESLPFGDNSFK. $+2 \mathrm{~b} 4$.light \\
\hline 174 & P42785.YYGESLPFGDNSFK.+2b6.light \\
\hline 175 & P42785.YYGESLPFGDNSFK. $+2 \mathrm{y} 10$. light \\
\hline 176 & P42785.YYGESLPFGDNSFK.+2y6.light \\
\hline 177 & P42785.YYGESLPFGDNSFK.+2y8.light \\
\hline 178 & P68871.EFTPPVQAAYQK.+2y10.light \\
\hline 179 & P68871.EFTPPVQAAYQK.+2y10+2.light \\
\hline 180 & P68871.EFTPPVQAAYQK.+2y8+2.light \\
\hline 181 & P68871.EFTPPVQAAYQK.+2y9.light \\
\hline 182 & P68871.EFTPPVQAAYQK.+2y9+2.light \\
\hline 183 & P68871.SAVTALWGK.+2b5.light \\
\hline 184 & P68871.SAVTALWGK.+2y5.light \\
\hline 185 & P68871.SAVTALWGK.+2y6.light \\
\hline 186 & P68871.SAVTALWGK.+2y7.light \\
\hline 187 & P98160.DFISLGLQDGHLVFR.+3y11+2.light \\
\hline 188 & P98160.DFISLGLQDGHLVFR.+3y12+2.light \\
\hline 189 & P98160.DFISLGLQDGHLVFR.+3y13+2.light \\
\hline 190 & P98160.DFISLGLQDGHLVFR.+3y5.light \\
\hline 191 & P98160.DFISLGLQDGHLVFR.+3y8.light \\
\hline 192 & P98160.LVSEDPINDGEWHR.+3y11+2.light \\
\hline 193 & P98160.LVSEDPINDGEWHR. +3 y $12+2$.light \\
\hline 194 & P98160.LVSEDPINDGEWHR.+3y3.light \\
\hline 195 & P98160.LVSEDPINDGEWHR.+3y6.light \\
\hline 196 & P98160.LVSEDPINDGEWHR.+3y7.light \\
\hline 197 & P98160.SIEYSPQLEDAGSR.+2b5.light \\
\hline 198 & P98160.SIEYSPQLEDAGSR.+2y10.light \\
\hline 199 & P98160.SIEYSPQLEDAGSR.+2y6.light \\
\hline 200 & P98160.SIEYSPQLEDAGSR.+2y7.light \\
\hline 201 & P98160.SIEYSPQLEDAGSR.+2y9.light \\
\hline 202 & sp|P02769|ALBU_BOVIN.HLVDEPQNLIK.+2b2.light \\
\hline 203 & sp|P02769|ALBU_BOVIN.HLVDEPQNLIK.+2b5.light \\
\hline 204 & sp|P02769|ALBU_BOVIN.HLVDEPQNLIK.+2y6.light \\
\hline 205 & sp|P02769|ALBU_BOVIN.HLVDEPQNLIK.+2y8.light \\
\hline 206 & sp|P02769|ALBU_BOVIN.HLVDEPQNLIK.+2y9.light \\
\hline 207 & sp|P02769|ALBU_BOVIN.LGEYGFQNALIVR.+2y10.light \\
\hline 208 & sp|P02769|ALBU BOVIN.LGEYGFQNALIVR.+2y6.light \\
\hline 209 & sp|P02769|ALBU_BOVIN.LGEYGFQNALIVR.+2y7.light \\
\hline 210 & sp|P02769|ALBU_BOVIN.LGEYGFQNALIVR.+2y8.light \\
\hline 211 & sp|P02769|ALBU_BOVIN.LGEYGFQNALIVR.+2y9.light \\
\hline 212 & sp|P02769|ALBU_BOVIN.LVNELTEFAK.+2b2.light \\
\hline 213 & sp|P02769|ALBU_BOVIN.LVNELTEFAK.+2y5.light \\
\hline 214 & sp|P02769|ALBU_BOVIN.LVNELTEFAK.+2y6.light \\
\hline 215 & sp|P02769|ALBU_BOVIN.LVNELTEFAK.+2y7.light \\
\hline
\end{tabular}


Table S11. Quantification results of the $17 \mathrm{BCa}$ related proteins by MRM.

\begin{tabular}{|c|c|c|c|c|c|c|}
\hline NO. & $\begin{array}{c}\text { Swissprot } \\
\text { number }\end{array}$ & $\begin{array}{c}\text { Protein } \\
\text { description }\end{array}$ & $\begin{array}{c}\text { Protein } \\
\text { name }\end{array}$ & $\begin{array}{l}\text { Log } 2 \text { fold changes } \\
\text { (BCa/Normal) }\end{array}$ & $\begin{array}{l}\text { up or down } \\
\text { in } \mathrm{BCa}\end{array}$ & p-value \\
\hline 1 & P68871 & $\begin{array}{l}\text { Hemoglobin } \\
\text { subunit beta }\end{array}$ & HBB & 2.96 & up & 0.001721744 \\
\hline 2 & P01024 & Complement C3 & $\mathrm{CO} 3$ & 2.15 & up & $2.12 \mathrm{E}-05$ \\
\hline 3 & P07195 & $\begin{array}{c}\text { L-lactate } \\
\text { dehydrogenase B } \\
\text { chain }\end{array}$ & LDHB & 1.36 & up & 0.011763245 \\
\hline 4 & P02768 & Serum albumin & ALBU & 0.88 & up & 0.008292831 \\
\hline 5 & P98160 & $\begin{array}{c}\text { Basement } \\
\text { membrane-specific } \\
\text { heparan sulfate } \\
\text { proteoglycan core } \\
\text { protein }\end{array}$ & PGBM & -1.10 & down & 0.006135468 \\
\hline 6 & P02751 & Fibronectin & FINC & -0.69 & down & 0.01904742 \\
\hline 7 & P12830 & Cadherin-1 & CADH1 & -0.90 & down & 0.02514274 \\
\hline 8 & P42785 & $\begin{array}{l}\text { Lysosomal Pro-X } \\
\text { carboxypeptidase }\end{array}$ & PCP & -0.86 & down & 0.019950388 \\
\hline 9 & P16070 & CD44 antigen & CD44 & -1.0 & down & 0.008630975 \\
\hline 10 & P16870 & Carboxypeptidase E & CBPE & -1.16 & down & 0.005695232 \\
\hline 11 & P02649 & Apolipoprotein E & APOE & 0.21 & $\# \mathrm{~N} / \mathrm{A}$ & 0.277437226 \\
\hline 12 & P05156 & $\begin{array}{l}\text { Complement factor } \\
\text { I }\end{array}$ & CFAI & 0.11 & \#N/A & 0.677263667 \\
\hline 13 & P12109 & $\begin{array}{l}\text { Collagen alpha- } \\
\text { 1(VI) chain }\end{array}$ & CO6A1 & -0.40 & $\# \mathrm{~N} / \mathrm{A}$ & 0.503999671 \\
\hline 14 & P04264 & $\begin{array}{l}\text { Keratin, type II } \\
\text { cytoskeletal } 1\end{array}$ & $\mathrm{~K} 2 \mathrm{C} 1$ & -0.14 & $\# \mathrm{~N} / \mathrm{A}$ & 0.522652877 \\
\hline 15 & P10253 & $\begin{array}{l}\text { Lysosomal alpha- } \\
\text { glucosidase }\end{array}$ & LYAG & -0.59 & \#N/A & 0.285042811 \\
\hline 16 & P01009 & Alpha-1-antitrypsin & A1AT & 0.56 & \#N/A & 0.156452139 \\
\hline 17 & P07339 & Cathepsin D & CATD & -0.43 & \#N/A & 0.190600604 \\
\hline
\end{tabular}


Table S12. ROC analysis results of the 10 potential BCa biomarkers.

\begin{tabular}{ccccccc}
\hline NO. & $\begin{array}{c}\text { Swiss-prot } \\
\text { number }\end{array}$ & $\begin{array}{c}\text { Protein } \\
\text { name }\end{array}$ & $\begin{array}{c}\text { AUC } \\
\text { value }\end{array}$ & T-test & Sensitivity & Specifity \\
\hline 1 & P68871 & HBB & 0.75 & 0.001788 & $62.50 \%$ & $78.26 \%$ \\
2 & P01024 & CO3 & 0.84 & 0.000025 & $87.50 \%$ & $78.26 \%$ \\
3 & P07195 & LDHB & 0.77 & 0.012455 & $83.33 \%$ & $65.22 \%$ \\
4 & P02768 & ALBU & 0.75 & 0.009128 & $87.50 \%$ & $65.22 \%$ \\
5 & P98160 & PGBM & 0.71 & 0.005243 & $83.33 \%$ & $60.87 \%$ \\
6 & P02751 & FINC & 0.67 & 0.018998 & $62.50 \%$ & $69.57 \%$ \\
7 & P12830 & CADH1 & 0.66 & 0.021281 & $70.83 \%$ & $78.26 \%$ \\
8 & P42785 & PCP & 0.68 & 0.014989 & $70.83 \%$ & $69.57 \%$ \\
9 & P16070 & CD44 & 0.67 & 0.010904 & $70.83 \%$ & $60.87 \%$ \\
10 & P16870 & CBPE & 0.69 & 0.013445 & $70.83 \%$ & $65.22 \%$ \\
Combination & CO3 and LDHB & 0.87 & 0.002672 & $87.00 \%$ & $79.12 \%$ \\
\hline
\end{tabular}


Table S13. MRM quantification results of the 10 potential BCa biomarkers in peptide level.

\begin{tabular}{|c|c|c|c|c|c|}
\hline NO. & peptide & sequence of peptide & $\begin{array}{l}\log 2 \\
\text { (Area) } \\
\text { of BCa }\end{array}$ & $\begin{array}{c}\log 2 \\
\text { (Area) } \\
\text { of } \\
\text { Normal }\end{array}$ & $\begin{array}{c}\text { Log2 fold } \\
\text { changes } \\
(\mathrm{B} / \mathrm{N})\end{array}$ \\
\hline 1 & HBB_pep1 & EFTPPVQAAYQK & 22.03 & 19.23 & 2.80 \\
\hline 2 & HBB_pep2 & SAVTALWGK & 22.08 & 19.16 & 2.92 \\
\hline 3 & CO3_pep1 & DFDFVPPVVR & 23.08 & 20.70 & 2.38 \\
\hline 4 & $\mathrm{CO} 3$ pep2 & QPSSAFAAFVK & 18.81 & 17.39 & 1.42 \\
\hline 5 & $\mathrm{CO} 3$ pep3 & SSLSVPYVIVPLK & 20.89 & 19.03 & 1.86 \\
\hline 6 & LDHB_pep1 & GLTSVINQK & 25.14 & 23.83 & 1.31 \\
\hline 7 & LDHB_pep2 & SLADELALVDVLEDK & 20.31 & 18.99 & 1.31 \\
\hline 8 & ALBU_pep1 & FQNALLVR & 28.97 & 27.86 & 1.11 \\
\hline 9 & ALBU_pep2 & LVNEVTEFAK & 29.22 & 28.35 & 0.87 \\
\hline 10 & PGBM_pep1 & DFISLGLQDGHLVFR & 24.83 & 25.88 & -1.04 \\
\hline 11 & PGBM_pep2 & LVSEDPINDGEWHR & 24.13 & 25.30 & -1.17 \\
\hline 12 & PGBM pep3 & SIEYSPQLEDAGSR & 18.44 & 19.47 & -1.03 \\
\hline 13 & FINC_pep1 & STTPDITGYR & 21.55 & 22.38 & -0.83 \\
\hline 14 & FINC_pep2 & VDVIPVNLPGEHGQR & 21.91 & 22.41 & -0.50 \\
\hline 15 & FINC_pep3 & VTWAPPPSIDLTNFLVR & 20.85 & 21.55 & -0.70 \\
\hline 16 & CADH1_pep1 & DTANWLEINPDTGAISTR & 19.54 & 20.09 & -0.55 \\
\hline 17 & CADH1_pep2 & GQVPENEANVVITTLK & 20.03 & 20.73 & -0.70 \\
\hline 18 & CADH1_pep3 & NTGVISVVTTGLDR & 21.01 & 21.99 & -0.97 \\
\hline 19 & PCP_pep2 & HLNFLTSEQALADFAELIK & 16.76 & 17.65 & -0.89 \\
\hline 20 & PCP pep2 & YYGESLPFGDNSFK & 18.36 & 19.05 & -0.69 \\
\hline 21 & CD44_pep1 & FAGVFHVEK & 21.73 & 22.87 & -1.14 \\
\hline 22 & CD44_pep2 & YGFIEGHVVIPR & 25.35 & 26.36 & -1.00 \\
\hline 23 & CBPE_pep1 & LTASAPGYLAITK & 18.17 & 19.20 & -1.03 \\
\hline 24 & CBPE_pep2 & NSLISYLEQIHR & 19.95 & 21.06 & -1.12 \\
\hline
\end{tabular}


Table S14. Disease-related information of the 10 potential BCa biomarkers summarized from references.

\begin{tabular}{|c|c|c|c|c|c|c|c|}
\hline NO. & $\begin{array}{l}\text { Protein } \\
\text { name }\end{array}$ & $\begin{array}{l}\text { Cancer } \\
\text { related }\end{array}$ & $\begin{array}{c}\mathrm{BCa} \\
\text { related }\end{array}$ & $\begin{array}{l}\text { Inflammation } \\
\text { related }\end{array}$ & $\begin{array}{l}\text { Kidney } \\
\text { disease } \\
\text { related }\end{array}$ & Location & Type(s) \\
\hline 1 & $\mathrm{CO} 3$ & YES & $\mathrm{NO}$ & YES & YES & $\begin{array}{l}\text { Extracellular } \\
\text { Space }\end{array}$ & peptidase \\
\hline 2 & LDHB & YES & YES & YES & YES & Cytoplasm & enzyme \\
\hline 3 & ALBU & YES & NO & YES & YES & $\begin{array}{c}\text { Extracellular } \\
\text { Space }\end{array}$ & transporter \\
\hline 4 & CD44 & YES & YES & YES & YES & $\begin{array}{l}\text { Plasma } \\
\text { Membrane }\end{array}$ & enzyme \\
\hline 5 & CDH1 & YES & YES & YES & YES & $\begin{array}{l}\text { Plasma } \\
\text { Membrane }\end{array}$ & other \\
\hline 6 & CBPE & YES & $\mathrm{NO}$ & YES & YES & Cytoplasm & peptidase \\
\hline 7 & FINC & YES & $\mathrm{NO}$ & YES & YES & $\begin{array}{c}\text { Extracellular } \\
\text { Space }\end{array}$ & enzyme \\
\hline 8 & HBB & YES & NO & YES & YES & Cytoplasm & transporter \\
\hline 9 & PGBM & YES & NO & NO & $\mathrm{NO}$ & $\begin{array}{l}\text { Extracellular } \\
\text { Space }\end{array}$ & enzyme \\
\hline 10 & PCP & NO & NO & YES & YES & Cytoplasm & peptidase \\
\hline
\end{tabular}

